\title{
Strukturdynamik wasserhaltiger Aggregate in Überschallexpansionen
}

Dissertation

zur Erlangung des mathematisch-naturwissenschaftlichen Doktorgrades „Doctor rerum naturalium" der Georg-August-Universität Göttingen

im Promotionsprogramm Chemie der Georg-August-University School of Science (GAUSS)

vorgelegt von

Julia Zischang

aus Celle

Göttingen, 2014 


\section{Betreuungsausschuss}

Prof. Dr. Martin A. Suhm,

Institut für Physikalische Chemie, Georg-August-Universität Göttingen

PD Dr. Thomas Zeuch,

Institut für Physikalische Chemie, Georg-August-Universität Göttingen

\section{Mitglieder der Prüfungskommission}

Referent: Prof. Dr. Martin A. Suhm, Institut für Physikalische Chemie, Georg-August-Universität Göttingen Korreferent: PD Dr. Thomas Zeuch,

Institut für Physikalische Chemie, Georg-August-Universität Göttingen

\section{Weitere Mitglieder der Prüfungskommission}

Prof. Dr. Burkhard Geil,

Institut für Physikalische Chemie, Georg-August-Universität Göttingen Jun.-Prof. Dr. Ricardo Mata,

Institut für Physikalische Chemie, Georg-August-Universität Göttingen

Prof. Dr. Jörg Schroeder,

Institut für Physikalische Chemie, Georg-August-Universität Göttingen Prof. Dr. Philipp Vana,

Institut für Physikalische Chemie, Georg-August-Universität Göttingen

Tag der mündlichen Prüfung: 23.04.2014 
If you want to have good ideas you must have many ideas. Most of them will be wrong, and what you have to learn is which ones to throw away.

Linus PAULing 

Diese Arbeit wäre nicht ohne das Mitwirken zahlreicher Menschen entstanden. An erster Stelle möchte ich mich herzlich bei Prof. Martin A. Suhm für die hervorragende Betreuung dieser Arbeit, seine Unterstützung, Diskussionsfreude und Begeisterungsfähigkeit bedanken. Ebenso gilt mein Dank PD Dr. Thomas Zeuch für sein reges Interesse und den beständigen Austausch zu dieser Arbeit.

Bei den derzeitigen und ehemaligen Mitgliedern der Suhm Group möchte ich mich für das entspannte und offene Arbeitsklima bedanken sowie für die hilfreichen Anmerkungen zu dieser Arbeit. Frau Katharina Meyer und Frau Christine E. Schiewer gilt mein Dank für ihre tatkräftige Hilfe in den frühen Stadien der Muesli- und Chilli-JetMessungen im Rahmen ihrer Bachelorarbeit bzw. ihres Forschungspraktikums. Ebenso möchte ich mich bei Dr. Tina Scharge für den ursprünglichen Aufbau des MuesliJets, bei Dr. Marija Nedić für die Vorarbeiten zur chemischen Bildgebung und bei Dr. Juhyon Lee für seine Erläuterungen zum Popcorn-Jet bedanken.

Stellvertretend für die Werkstätten, ohne die unsere experimentelle Arbeit nicht möglich wäre, geht mein Dank an Frau Annika von Roden, Herrn Volker Meyer und Herrn Andreas Knorr für ihr Fachwissen, ihren Ideenreichtum und ihre Hilfsbereitschaft.

Bei Prof. Andrey F. Vilesov möchte ich mich für die Kooperationsmöglichkeit und die Einblicke in die Welt der Heliumnanotröpfchen bedanken.

Keine Forschung ohne Finanzierung - vielen Dank an die Deutsche Telekom Stiftung für das Doktorandenstipendium, die Ermöglichung von Konferenzreisen und das Interesse nicht nur an Forschungsergebnissen, sondern auch an den Menschen, die sie erbringen.

An letzter Stelle, wenn auch nicht zuletzt, gilt mein Dank meiner Familie und meinen Freunden für ihre Unterstützung, Aufmunterungen und Geduld. 



\section{Inhaltsverzeichnis}

1 Einleitung 1

2 Grundlagen 3

2.1 Intermolekulare Wechselwirkungen . . . . . . . . . . . . . . . 3

2.2 FTIR-Spektroskopie von Überschall-Schlitzdüsenexpansionen . . . . . . 4

2.3 Struktur von Überschallexpansionen durch Schlitzdüsen . . . . . . . . . 7

2.4 Eigenschaften von Multidüsenexpansionen . . . . . . . . . . . . . . . . . 9

2.5 Chemische Bildgebung von Überschallexpansionen . . . . . . . . . . . . 11

2.6 Temperaturabschätzung in Überschallexpansionen . . . . . . . . . . . . 14

3 Experimentelles $\quad 18$

3.1 Genereller Aufbau der Jet-Apparaturen . . . . . . . . . . . . . . . . . . . 18

3.1.1 Überschallexpansion . . . . . . . . . . . . . . . . . . . . . . 18

3.1.2 Spektrenaufnahme und Synchronisation . . . . . . . . . . . . . . 19

3.1.3 Datenverarbeitung .................. . . 20

3.2 Muesli-Jet-Apparatur . . . . . . . . . . . . . . . . 21

3.2.1 Grundidee . . . . . . . . . . . . . . . . . 21

3.2 .2 Aufbau . . . . . . . . . . . . . . . . . . . 21

3.2.3 Düsengeometrien . . . . . . . . . . . . . . . . . . 24

3.2 .4 Vorversuche . . . . . . . . . . . . . . . 25

3.3 Popcorn-Jet-Apparatur . . . . . . . . . . . . . . . . . . . 34

3.3.1 Grundidee . . . . . . . . . . . . . . . . 34

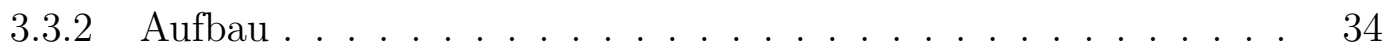

3.4 Chilli-Jet-Apparatur . . . . . . . . . . . . . . . 36

3.4 .1 Grundidee . . . . . . . . . . . . . . . . 36

3.4 .2 Aufbau ............................ 36

3.4.3 Düsenkonstruktion . . . . . . . . . . . . . . . . . . 37

3.4.4 Datenaufnahme . . . . . . . . . . . . . . . . . . 39

3.4.5 Datennachbehandlung . . . . . . . . . . . . . 40

3.4.6 Charakterisierung des pixelübergreifenden Rauschens . . . . . . 42

4 Aggregationsverhalten in Multischlitzdüsenexpansionen 45

4.1 Ein Modellsystem - Methanol und tert-Butylalkohol . . . . . . . . . . . 45

4.1.1 Bildung gemischter Aggregate . . . . . . . . . . . . . . . . . 46 
4.1 .2 Isotopenaustausch _. . . . . . . . . . . . . . . . 52

4.2 Warme Expansionen - Wasser . . . . . . . . . . . . . . 55

4.2.1 Monomer-Rotationstemperaturen . . . . . . . . . . . 58

4.2.2 Einfluss der Temperatur auf Aggregatstrukturen . . . . . . . . . 65

4.3 Bildung von Mischaggregaten - Wasser und Deuteriumoxid . . . . . . . 76

4.4 Zusammenfassung und Ausblick . . . . . . . . . . . . . . . . 90

5 Donor-Akzeptor-Präferenzen $\quad 92$

5.1 Wasser und Imidazol . . . . . . . . . . . . . . . . . . . . . . . . . 93

5.2 Wasser und 4-/5-Phenylimidazol . . . . . . . . . . . . . . . . . . . . 104

5.3 Zusammenfassung und Ausblick . . . . . . . . . . . . . 109

6 Infrarotspektroskopische Bildgebung von Überschallexpansionen $\quad 111$

6.1 Distickstoffmonoxid $\left(\mathrm{N}_{2} \mathrm{O}\right) \ldots \ldots \ldots \ldots \ldots$

6.2 Gepulste Expansionen . . . . . . . . . . . . . . . . . . . . . 112

6.3 Kontinuierliche Expansionen . . . . . . . . . . . . . . . . . . . . . . 119

6.3 .1 Dichteverlauf . . . . . . . . . . . . . . . . . 119

6.3 .2 Temperaturverlauf . . . . . . . . . . . . . . . . . . . 124

6.4 Zusammenfassung und Ausblick . . . . . . . . . . . . . 127

$\begin{array}{lll}7 & \text { Zusammenfassung und Ausblick } & 130\end{array}$

A Anhang I

A.1 Publikationen . . . . . . . . . . . . . . . . I

A.2 Verwendete Chemikalien . . . . . . . . . . . . . . . I I

A.3 Spektrenverzeichnis . . . . . . . . . . . . . . . . . II

A.4 Detaillierte Ergebnisse der quantenchemischen Rechnungen . . . . . . . V

A.5 Experimentelle Parameter . . . . . . . . . . . . . . . . IX

A.5.1 Muesli-Jet ....................... IX

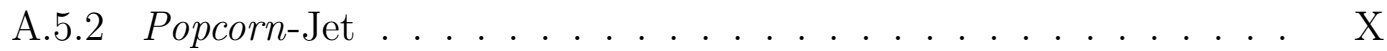

A.5.3 Chilli-Jet .................. XI

A.6 Pulssynchronisation . . . . . . . . . . . . . . . . . X XI

A.6.1 TRS-Methode ...................... XI

A.6.2 Puls- und Verzögerungszeiten . . . . . . . . . . . . . XII

A.7 Makros . . . . . . . . . . . . . . . . . . . . . XIII

A.7.1 Muesli-Jet ................... XIII

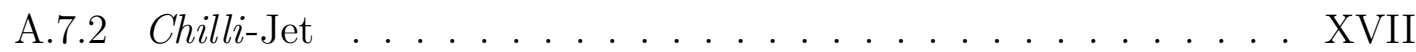

$\begin{array}{ll}\text { Literaturverzeichnis } & \text { XXVIII }\end{array}$ 


\section{Einleitung}

Wasser $\left(\mathrm{H}_{2} \mathrm{O}\right)$ gehört zu den häufigsten Molekülen auf der Erde und sein flüssiges Vorliegen ist eine der Voraussetzungen für das Leben auf unserem Planeten. Eben dieses Vorliegen als Flüssigkeit bei Standardbedingungen trotz geringer molarer Masse wird dadurch ermöglicht, dass jedes $\mathrm{H}_{2} \mathrm{O}$-Molekül jeweils zwei Wasserstoffbrückendonor- und -akzeptorfunktionalitäten aufweist. So sind Wassermoleküle in der Lage, Netzwerke miteinander interagierender Wasserstoffbrückenbindungen auszubilden, wodurch die Kondensation befördert wird. ${ }^{1}$

In der Natur tritt Wasser nicht nur makroskopisch auf, sondern auch in der Form kleiner Aggregate und einzelner Moleküle. Wasseraggregate tragen vermutlich zur Absorption von Infrarotstrahlung in der Atmosphäre bei und beeinflussen somit das Strahlungsgleichgewicht der Erde. ${ }^{2}$ Solche Cluster aus wenigen Wassermolekülen sind unter den kalten Bedingungen von Überschallexpansionen spektroskopisch recht gut untersucht $^{3,4,5}$, der Nachweis in der Gasphase bei $300 \mathrm{~K}$ gestaltet sich jedoch problematischer und ist für das kleinste Wasseraggregat, das Dimer, erst kürzlich überzeugend durch Millimeterwellen-Spektroskopie von konzentriertem Wasserdampf erbracht worden. ${ }^{6}$ Ein schwingungsspektroskopischer Beleg steht jedoch noch aus, da hier die Aggregatbanden zum großen Teil vom breiten, komplexen Spektrum des Monomers überlagert werden. Kapitel 4.2 beschäftigt sich daher mit den Infrarotspektren thermisch leicht angeregter Wasseraggregate nahe am evaporativen Limit, um so einen Zwischenschritt zur letztendlichen schwingungsspektroskopischen Identifizierung in der Gasphase zu liefern. Die warmen Cluster werden dabei in Multischlitzdüsenexpansionen generiert, die eine bislang wenig gebräuchliche, jedoch vielseitige experimentelle Methode zur Untersuchung der Eigenschaften von Molekülaggregaten darstellen.

Auch die erfolgreiche Strukturaufklärung thermisch angeregter, kleiner Wasseraggregate liefert jedoch noch keine Hinweise auf Bildungsmechanismen und dynamische Prozesse innerhalb der Cluster. Daher wurde eine neuartige Multischlitzdüsengeometrie entworfen, die für die Erzeugung gemischter Aggregate optimiert ist. Diese wurde unter anderem zur Untersuchung synchroner Expansionen von Wasser und Deuteriumoxid $\left(\mathrm{D}_{2} \mathrm{O}\right)$ eingesetzt, wobei die Zusammensetzung der innerhalb der Expansion generierten Aggregate Aufschluss über energetische Bevorzugungen einzelner Aggregattypen und über Isotopenaustauschprozesse gibt.

Neben diesen reinen Wasseraggregaten sind in der Natur auch die isolierten Wechselwirkungen einzelner Wassermoleküle mit biologisch relevanten Substanzen von Bedeutung. Ein Beispiel hierfür stellen die Aktivzentren von Serinproteasen dar, in denen die Interaktion eines Wassermoleküls mit der Imidazolyl-Seitenkette eines Histidins 
einen Ladungstransport ermöglicht, der für die katalysierte Peptidspaltung essentiell ist. ${ }^{7,8}$ Während die Strukturaufklärung von Proteinen üblicherweise durch Röntgenbeugungsmethoden erfolgt, ist hiermit die Analyse einzelner Wasserstoffbrückenbindungen aufgrund der geringen Streuamplitude von Wasserstoff häufig problematisch. ${ }^{9}$ Daher werden in dieser Arbeit exemplarisch die isolierten Wechselwirkungen von Imidazol beziehungsweise 4-/5-Phenylimidazol mit Wasser bezüglich des bevorzugten Bindungsmotivs betrachtet. So können wertvolle Informationen für die Mechanismenaufklärung enzymatisch katalysierter Prozesse gewonnen werden.

Die vorgestellten Untersuchungen erfolgen dabei mittels Fourier-TransformationsInfrarot-Spektroskopie an Überschall-Schlitzdüsenexpansionen. ${ }^{10}$ Dabei werden in den Expansionen unter überwiegend thermodynamischer Kontrolle isolierte, kalte Molekülaggregate gebildet. Mittels der Absorption breitbandiger, infraroter Strahlung werden Schwingungsspektren der Cluster erhalten und aus den Positionen, Intensitäten und Formen der beobachteten Banden Rückschlüsse auf die Strukturen der in der Expansion enthaltenen Moleküle und Aggregate gezogen.

Bei der Verwendung von Einzelelement-Infrarotdetektoren werden aufgrund des relativ hohen Durchmessers des Infrarotstrahls immer über einen größeren Bereich gemittelte spektrale Daten erhalten, die die Heterogenität und Dynamik innerhalb der Expansionen nur sehr eingeschränkt wiedergeben können. Daher wurde im Rahmen dieser Arbeit zusätzlich eine Apparatur zur chemischen Bildgebung von Überschallexpansionen entwickelt. Die synchron gemessenen, ortsaufgelösten Spektren können Zugang zu Informationen über den Dichte- und Temperaturverlauf, Aggregatbildungs-, Zerfallsund Umlagerungsdynamik bieten. Als erstes Testsystem für derartige Betrachtungen wurde der starke IR-Absorber Lachgas $\left(\mathrm{N}_{2} \mathrm{O}\right)$ gewählt.

Nach einem kurzen Überblick über die theoretischen Grundlagen zu den durchgeführten experimentellen Arbeiten in Kapitel 2 werden in Kapitel 3 die überwiegend im Rahmen dieser Arbeit entwickelten Apparaturen beschrieben und charakterisiert. Im Anschluss thematisiert Kapitel 4.1 zunächst Multischlitzdüsenexpansionen von Alkoholen als Modellsysteme für Wasser, bevor in Abschnitt 4.2 auf die Strukturen thermisch angeregter Wasseraggregate und in Abschnitt 4.3 auf die synchronen Expansionen von $\mathrm{H}_{2} \mathrm{O}$ und $\mathrm{D}_{2} \mathrm{O}$ eingegangen wird. Die Bestimmung der bevorzugten Wechselwirkungsmotive von Wasser und Imidazol sowie von Wasser und 4-/5-Phenylimidazol wird in Kapitel 5 behandelt. Die Vorstellung der neuen Methode zur infrarotspektroskopischen Bildgebung von Überschallexpansionen und ein Überblick über die daraus zugänglichen Informationen erfolgen in Kapitel 6. Schließlich werden in Kapitel 7 die erhaltenen Ergebnisse zusammengefasst und ein Ausblick auf zukünftige experimentelle Schritte gegeben. 


\section{Grundlagen}

\subsection{Intermolekulare Wechselwirkungen}

Die Summe aller attraktiven und repulsiven Kräfte, die zwischen einzelnen Molekülen wirken können und nicht zur Ausbildung chemischer Bindungen führen, wird als „zwischenmolekulare Wechselwirkungen“ bezeichnet. Sie bewirken sowohl die Abweichungen gasförmiger Stoffe vom idealen Verhalten bis hin zur Clusterbildung und Kondensation als auch die endliche Kompressibilität von Substanzen. Mit einer typischen Größenordnung von $1-25 \mathrm{~kJ} \mathrm{~mol}^{-1}$ sind zwischenmolekulare Wechselwirkungen dabei deutlich schwächer als die meisten chemischen Bindungen. ${ }^{11}$

Attraktive intermolekulare Kräfte werden häufig in Elektrostatik, Induktion und Dispersion unterteilt. Informationen zu ihren Ursachen, Abstandsabhängigkeiten und typischen Größenordnungen können den Referenzen 1,11,12,13 entnommen werden. Repulsive Kräfte zwischen Molekülen treten überwiegend bei sehr kleinen Abständen auf und basieren im Wesentlichen auf der Abstoßung, die einerseits durch elektrostatische Repulsion der Atomkerne (Coulomb-Abstoßung) als auch durch eine Überlappung der Wellenfunktionen der Elektronen (Pauli-Abstoßung) auftritt. ${ }^{11,12}$

Obwohl für energetische Berechnungen von Molekülaggregaten häufig angenommen wird, dass die gesamte Wechselwirkung aus der Summe aller Paarwechselwirkungen sinnvoll genähert werden kann, müssen in Abhängigkeit vom untersuchten System für eine hinreichende Beschreibung auch Mehrkörperwechselwirkungen berücksichtigt werden. ${ }^{11,14,15}$

Eine besondere Form der intermolekularen Wechselwirkungen stellen die Wasserstoffbrückenbindungen dar. Während sich eine frühe Beschreibung durch Pauling noch rein auf elektrostatische Effekte stützte ${ }^{16}$, besagt die moderne, allgemeinere IUPACDefinition von 2011: „The hydrogen bond is an attractive interaction between a hydrogen atom from a molecule or a molecular fragment $X-H$ in which $X$ is more electronegative than $H$, and an atom or a group of atoms in the same or a different molecule, in which there is evidence of bond formation." 17 Hierbei darf die attraktive Wechselwirkung nicht vorwiegend durch Dispersion hervorgerufen sein. Als Atomsorten X mit höherer Elektronegativität als Wasserstoff kommen C, N, O, F, P, S, Cl, Se, Br und I in Frage. ${ }^{18}$ Diese können ebenfalls Akzeptoratome darstellen, wobei auch Gruppen mit relativ hoher Elektronendichte wie $\pi$-Systeme als Akzeptoren in Wasserstoffbrückenbindungen fungieren können. ${ }^{1}$ Die Beiträge der Elektrostatik und Repulsion zur Bindungsenergie von Wasserstoffbrückenbindungen liegen jeweils etwa in der gleichen Größenordnung 
wie die Gesamtstärke (jedoch mit unterschiedlichen Vorzeichen); Induktion, Dispersion und Ladungstransfereffekte summieren sich ebenfalls auf einen ähnlichen Betrag, wobei der Dispersionsanteil häufig als größter anzusehen ist. ${ }^{11}$

Wasserstoffbrückenbindungen $\mathrm{X}-\mathrm{H} \cdots \mathrm{Y}$ sind stark gerichtet ${ }^{17,18}$ und ihre Stärke in der Größenordnung weniger $k_{\mathrm{B}} T$ bei $300 \mathrm{~K}$ führt zu der Kombination aus ausreichend stabiler Struktur bei gleichzeitig leichter Spaltbarkeit, die vielen Biomakromolekülen ihre Struktur und Funktion ermöglicht ${ }^{1,19}$ und zudem das für alles Leben notwendige flüssige Vorliegen von Wasser bei Umgebungstemperatur und -druck bewirkt. ${ }^{1}$

Aufgrund der in einer Wasserstoffbrückenbindung $\mathrm{X}-\mathrm{H} \cdots \mathrm{Y}$ verlängerten $\mathrm{X}-\mathrm{H}-\mathrm{Bin}-$ dung weisen sie (mit wenigen Ausnahmen ${ }^{20}$ ) in Infrarotspektren im Vergleich zu freien $\mathrm{X}-\mathrm{H}$-Bindungen üblicherweise kleinere Streckschwingungsfrequenzen auf (Rotverschiebung). ${ }^{20}$ Gleichzeitig erfolgt eine deutliche Verstärkung der Intensität der Streckschwingungsbande des gebundenen $\mathrm{X}-\mathrm{H}$-Oszillators aufgrund der Abhängigkeit des in Y induzierten Dipolmoments von der Position des Wasserstoffatoms und des damit einhergehenden verstärkten Übergangsdipolmoments. ${ }^{11,18,21}$

Bei Systemen mit mehreren Wasserstoffbrückenbindungen ist eine Berechnung der Gesamtwechselwirkungsenergien aus paarweisen Energien häufig nicht möglich. Grund kann eine Schwächung der Bindungen durch Konkurrenz um Elektronendichte (Antikooperativität) sein oder eine gegenseitige Verstärkung, die häufig in wasserstoffbrückengebundenen Ringstrukturen auftritt (Kooperativität). ${ }^{22}$

\subsection{FTIR-Spektroskopie von Überschall- Schlitzdüsenexpansionen}

Bei der Untersuchung spektraler Eigenschaften von isolierten Molekülen oder Molekülaggregaten bieten Überschallexpansionen deutliche Vorteile gegenüber Gasphasenuntersuchungen. Experimentell werden Überschallexpansionen dadurch generiert, dass ein Gas oder Gasgemisch von einem Reservoir höheren Drucks durch eine Düse in ein Volumen geringeren Drucks expandiert wird. ${ }^{23,24,25}$ Ist die Dimension der Düse dabei deutlich größer als die mittlere freie Weglänge im Gas unter Stagnationsbedingungen $^{26,27}$ und das Verhältnis von Reservoir- zu Hintergrunddruck ausreichend groß ${ }^{24,26}$, so findet zunächst eine näherungsweise isentrope Expansion des Gases in das Volumen der Expansionskammer statt. Aufgrund der hohen Anzahl von Stößen in der Region noch hoher Dichte bei geringen Düsenabständen erfolgt hierbei eine Energieumverteilung aus den verschiedenen Freiheitsgraden der Moleküle in die Translation in Ausbreitungsrichtung. Dies führt sowohl zu einer Beschleunigung als auch zu einer Abkühlung des Gasstroms und so auch zu einer Absenkung der lokalen Schallgeschwindigkeit. ${ }^{24}$ Aufgrund der abnehmenden Dichte des Gases entlang der Expansion verringert sich auch die Anzahl der Stöße, so dass die unterschiedliche Effizienz der stoßinduzierten Kühlung für verschiedene molekulare Freiheitsgrade zu unterschiedlichen Temperaturen 
für die Translation, Rotation und die verschiedenen Schwingungsmoden führt. ${ }^{24,26,28,29}$ Hierdurch und aufgrund der geringen Relativgeschwindigkeit der Moleküle zueinander in der Expansion gilt $T_{\text {trans }}<T_{\text {rot }}<T_{\text {vib }}$. Die Besetzung verschiedener energetischer Zustände weicht daher von einer Boltzmannverteilung $\mathrm{ab}^{30}$, Überschallexpansionen von Gasen stellen also eine Nicht-Gleichgewichtsmethode dar.

Bei ausreichender Dichte und vorliegenden intermolekularen Wechselwirkungen werden zu Anfang der Expansion Molekülcluster gebildet. Aufgrund des schnellen Dichteabfalls und der hierdurch fehlenden Stoßpartner in düsenferneren Regionen können diese Aggregate bestehen bleiben ${ }^{24,26,31}$ und so spektroskopisch untersucht werden. Menge und Größe der gebildeten Cluster können durch Wahl der Expansionsparameter wie Düsengeometrie, Reservoirdruck, Substanztemperatur und Trägergas gesteuert werden. ${ }^{23,32}$ Hierbei werden als Trägergase zur Verdünnung der untersuchten Substanzen zumeist Edelgase verwendet, da diese keine eigenen Rotations- und Schwingungsfreiheitsgrade aufweisen und so die Kühleffizienz der Probenmoleküle im Jet erhöht wird. ${ }^{26,27}$ Insbesondere Helium findet häufig Einsatz, da es nahezu keine Komplexe mit der Probensubstanz bildet. ${ }^{26}$ Eine Ausbreitungsgeschwindigkeit der Expansion oberhalb der lokalen Schallgeschwindigkeit (Mach-Zahl > 1) schließt zudem eine Beeinflussung der Cluster durch Moleküle und Bedingungen außerhalb der Expansionszone aus, da von dort eine Informationsübertragung maximal mit Schallgeschwindigkeit erfolgen kann. Durch eine Erhöhung des Druckquotienten vor $\left(p_{0}\right)$ und hinter $\left(p_{\mathrm{BG}}\right)$ der Düse $\frac{p_{0}}{p_{\mathrm{BG}}}$ entsteht eine ausreichend große Zone freier Expansion um spektroskopische Untersuchungen an kalten Molekülaggregaten vornehmen zu können. ${ }^{24,25,26}$ Aufgrund der fehlenden Beeinflussung der Eigenschaften durch äußere Bedingungen stellen Molekülcluster ideale Systeme für einen Vergleich mit quantenmechanischen Rechnungen dar.

Für die Aufrechterhaltung des Druckgefälles zwischen Reservoir und Expansionskammer ist eine entsprechende Pumpleistung erforderlich. Bei den für eine ausgeprägte Clusterbildung großen Düsendimensionen und hohen Reservoirdrücken und den damit verbundenen Gasdurchsätzen kann die Anforderung an das Pumpensystem durch einen gepulsten Betrieb der Expansionen gesenkt werden. Andersherum ermöglicht bei einer bestehenden Pumpleistung ein gepulster Betrieb auch einen höheren Gasdurchsatz und somit größere Substanzdichten und die damit verbundenen höheren Signalintensitäten in den Spektren. ${ }^{24,27}$ Dabei ist sowohl eine Synchronisation der Gaspulse mit der Spektrenaufnahme möglich als auch ein asynchroner Betrieb. ${ }^{33,34}$

Eine weitere Erhöhung der Absorbanzen kann durch den Einsatz von Schlitz- anstelle der häufiger verwendeten Lochdüsen erreicht werden. In Schlitzdüsenexpansionen erfolgt einerseits der Dichteabfall hinter der Düse linear (anstelle von quadratisch) ${ }^{30,32}$, was neben der allgemeinen Erhöhung der Signalintensitäten im Spektrum auch zu einer höheren Anzahl von Stößen und somit auch zu ausgeprägterer Aggregation ${ }^{24,35}$ und bei hinreichender Verdünnung im Trägergas zu einer stärkeren Kühlung der translatorischen und rotatorischen Freiheitsgrade ${ }^{24}$ führt. Für die Absorptionsspektroskopie ist 
zudem eine größere geometrische Überlappung von Expansion und Lichtstrahl realisierbar ${ }^{30,32}$ und eine Vergrößerung der Düsenöffnung zur Erhöhung des Absorptionswegs ist - im Gegensatz zu einer Lochdüsen-Vergrößerung - mittels einer Verlängerung der Schlitzdüse sowohl effizienter im Bezug auf den Gasverbrauch als auch ohne größeren Einfluss auf die Beschaffenheit der gebildeten Cluster möglich. ${ }^{24,32,36}$

Generell sind bei Überschallexpansionen aufgrund der geringen Temperaturen nur wenige energetische Zustände populiert, sodass sich schmale spektrale Banden ergeben, die auch die getrennte Analyse nahe beieinander liegender Signale ermöglichen. ${ }^{24,27,30}$ Für hochauflösende Spektren bieten Schlitzdüsenexpansionen durch vermehrte Stöße parallel zur Düse den zusätzlichen Vorteil der Verringerung von Geschwindigkeitskomponenten in dieser Richtung, so dass auch die Dopplerbreite der spektralen Banden geringer ist als beim Einsatz von Lochdüsen. ${ }^{32,35,37}$

In dieser Arbeit wurde zur Untersuchung von Molekülaggregaten im Überschallstrahl die Methode der direkten Absorptionsspektroskopie von infrarotem (IR) Licht gewählt. Mit ihr können Molekülschwingungen untersucht werden und hierdurch Rückschlüsse auf deren Strukturen und Eigenschaften gezogen werden. ${ }^{38} \mathrm{Im}$ Gegensatz zur Rotationsspektroskopie mit Mikrowellen, die häufig noch detailliertere Strukturinformationen liefert, ist die IR-Spektroskopie nicht auf ein permanentes Dipolmoment der untersuchten Substanzen angewiesen ${ }^{39}$, sondern ermöglicht eine Betrachtung aller Schwingungen, die mit einem nicht verschwindenden Übergangsdipolmoment einhergehen. ${ }^{38}$ Zusätzlich profitiert sie bei wasserstoffbrückengebundenen Systemen von einer hohen Bandenintensität der gebundenen $\mathrm{X}-\mathrm{H}$ Streckschwingungsfundamentalen $(\mathrm{X}=\mathrm{N}, \mathrm{O}) .{ }^{40}$

Für die synchrone Untersuchung eines breiten spektralen Bereichs, wie sie hier angestrebt wurde, bietet sich zudem die Fourier-Transformations-Infrarot (FTIR) Spektroskopie an. Durch Einsatz einer Breitband-Lichtquelle und interferometrische Modulation des IR-Lichts können aufgrund von Multiplexvorteil ${ }^{36}$ und hohem Lichtdurchsatz relativ rauscharme Spektren mit unproblematischer Frequenzkalibrierung ${ }^{30}$ aufgenommen werden. Der Einsatz einer inkohärenten Lichtquelle verhindert zudem die Verfälschung relativer Intensitäten durch nichtlineare Effekte ${ }^{30,32,39}$ und erhöht somit die Vergleichbarkeit mit theoretischen Intensitäten aus quantenmechanischen Berechnungen. Der relativ große Durchmesser des Lichtstrahls im Zentimeterbereich führt allerdings zu Einbußen bei der räumlichen Präzision, wodurch zwingend verschiedene Zonen der Expansion gleichzeitig untersucht werden.

Die Fourier-Transformations-Infrarot-Spektroskopie von Schlitzdüsen-Überschallexpansionen bietet somit eine universell einsetzbare Kombination für die Struktur- und Eigenschaftsaufklärung von isolierten Molekülaggregaten ${ }^{10}$ unter Vermeidung von Matrixeffekten. ${ }^{41}$ Die untersuchten Systeme befinden sich hierbei jedoch nicht im thermodynamischen Gleichgewicht ${ }^{30,42}$, so dass eine Annahme von Boltzmannverteilungen für Häufigkeitsanalysen stets unter Vorbehalt erfolgen muss. 


\subsection{Struktur von Überschallexpansionen durch Schlitzdüsen}

Überschallexpansionen weisen verschiedene Zonen auf, die sich in ihren Temperaturen, Strömungsgeschwindigkeiten, der Gasdichte sowie dem Ausmaß an Aggregatbildung unterscheiden. Direkt hinter dem Düsenausgang breitet sich das Gas ins Vakuum aus, seine Viskosität und der Wärmetransfer zwischen Gasfluss und Umgebung werden vernachlässigbar. Die Expansion kann daher als adiabatisch isentrop beschrieben werden. ${ }^{24,28}$ Bei der Ausbreitung nehmen, wie in Abschnitt 2.2 beschrieben, Druck und Temperatur ab. Bei der Expansion kondensierbarer Substanzen kann sich so eine Übersättigung im Gasgemisch einstellen, die bei Stößen der Moleküle zur Aggregation führt. ${ }^{24}$ Sinkt die Stoßzahl im weiteren Verlauf der Expansion aufgrund des Dichteabfalls, geht der zuvor als Kontinuumsströmung beschreibbare Freistrahl in eine Molekularströmung über. Dieser Bereich wird auch als Ruhezone bezeichnet. ${ }^{24,28}$ Gleichzeitig endet die durch Energieumwandlung aus den Freiheitsgraden der Moleküle resultierende Beschleunigung des Flusses, die Temperatur sinkt nicht weiter und eine terminale Machzahl wird erreicht. ${ }^{28}$

Da die Expansion sich mit Überschallgeschwindigkeit und somit schneller als die Störungen, die sie im Hintergrundgas hervorruft, ausbreitet, kommt es zu einer Überexpansion, die Dichte in der Expansion unterschreitet die des Hintergrundgases. ${ }^{24}$ Hierdurch gewinnen jenseits der Ruhezone Stöße von Molekülen aus der Expansion mit denen aus dem Hintergrundgas an Bedeutung und brechen die enge Energieverteilung des Freistrahls. ${ }^{28}$ Der Gasfluss wird somit irreversibel, die Entropie und die Temperatur steigen und weniger stabile Molekülaggregate dissoziieren. Derartige Zonen, in denen sich innerhalb einer geringen räumlichen Ausdehnung thermodynamische Eigenschaften und Strömungsparameter einer Expansion drastisch ändern, werden als Stoßfronten bezeichnet. Die genauen Prozesse in solchen Stoßfronten sowie deren Dicke und Struktur hängen maßgeblich vom Stagnationsdruck des expandierten Gases, der Dichte des Hintergrundgases sowie der Düsengeometrie ab. ${ }^{28}$

Die Struktur von Schlitzdüsenexpansionen ist schematisch in Abbildung 2.1 a dargestellt, experimentelle Beispiele sind in Abbildung $2.1 \mathrm{~b}$ und c gezeigt. Es treten im Wesentlichen zwei Typen von Stoßfronten auf, die den isentropen Kern der Expansionen umgeben. Die normale oder frontale Stoßfront ist nahezu planar und befindet sich senkrecht zur Ausbreitungsrichtung der Expansion. Sie führt zu einer Abbremsung des Gasflusses unterhalb der Schallgeschwindigkeit und wird häufig auch unpräzise als Machscheibe (die nur bei achsensymmetrischen Expansionen auftritt) bezeichnet. Seitlich wird die Zone freier Expansion durch laterale Stoßfronten begrenzt, die jedoch eine geringere Verlangsamung der Ausbreitung bewirken. Während die von den langen Seiten der Schlitzdüse ausgehenden Fronten zunächst divergent verlaufen, konvergieren die seitlichen Stoßfronten von den Schlitzenden aus und können sich in Abhängigkeit 


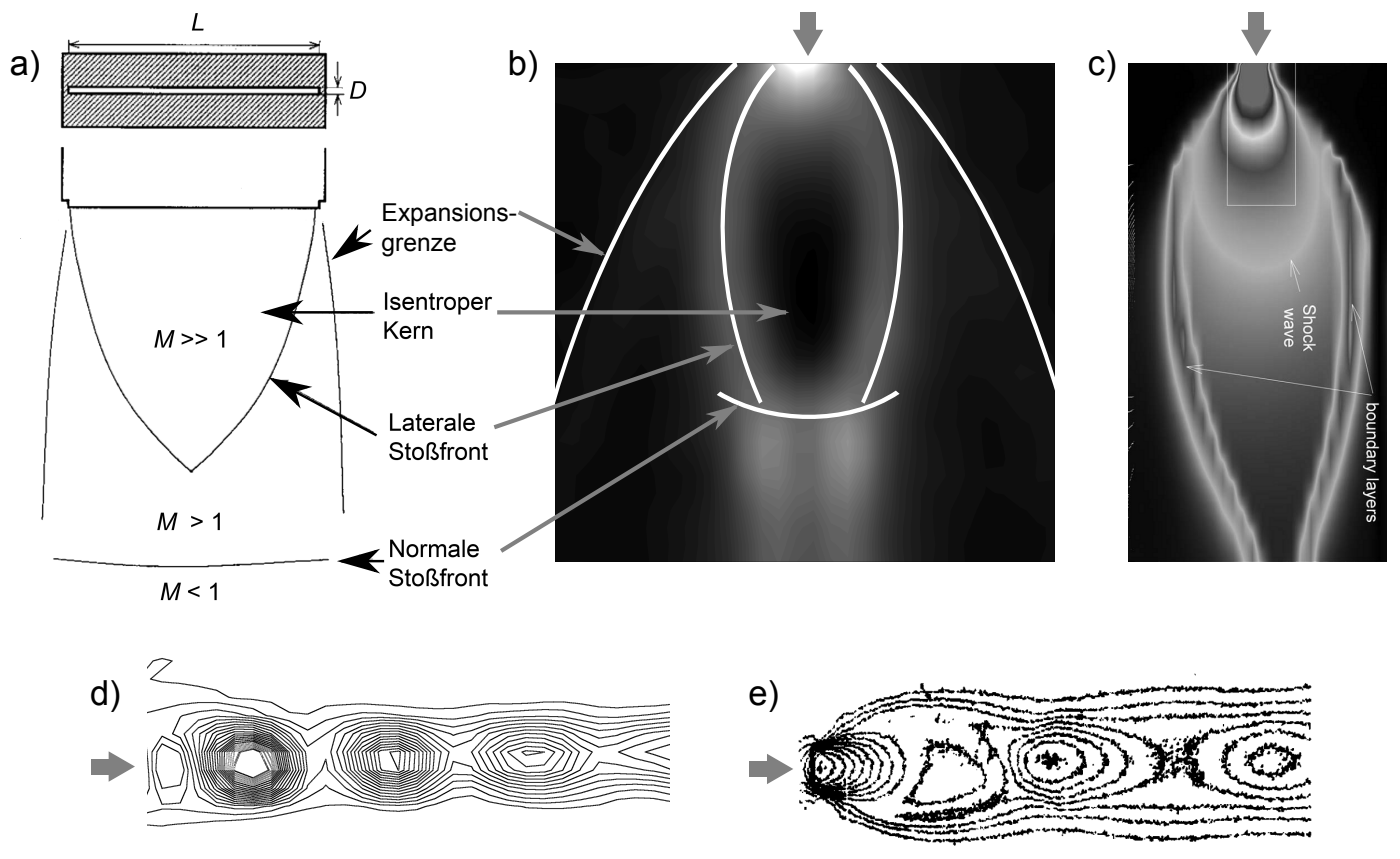

Abbildung 2.1: Struktur von Überschallexpansionen, dicke graue Pfeile kennzeichnen die Düsenausgangspositionen. a) Schematischer Aufbau von Expansionen aus Schlitzdüsen aus Referenz 24, Abb. 5; M: Machzahl. b) Dichteverlauf einer Chilli-Jet-Expansion, helle Regionen und weiße Hilfslinien kennzeichnen Zonen hoher Dichte. c) Druckverlauf einer Schlitzdüsenexpansion aus Ref. 43, Abb. 1. Helle Regionen kennzeichnen Zonen hohen Drucks. d) Isopyknen einer Chilli-Jet-Expansion bei erhöhtem Hintergrunddruck. e) Isopyknen einer Lochdüsen-Plasmaexpansion aus Abb. 4a, Ref. 44. Abbildungen b), c) und d) sind gegenüber a) um $90^{\circ}$ gedreht dargestellt.

von der Düsengeometrie und den vorliegenden Drücken vor oder hinter der normalen Stoßfront kreuzen. ${ }^{24,28}$

Der Abstand $y_{\mathrm{M}}$ der (ersten) normalen Stoßfront zum Düsenausgang ist abhängig von dem Verhältnis zwischen Stagnations- $\left(p_{0}\right)$ und Hintergrunddruck $\left(p_{\mathrm{BG}}\right)$ sowie von der Schlitzdüsenlänge $(L)$ und -breite $(D)$. Für den Grenzfall einer unendlich langen Schlitzdüse, die somit frei von Effekten durch die Düsenenden ist, wurde der empirische Zusammenhang

$$
y_{\mathrm{M}}=1.23 \cdot D \cdot\left(\frac{p_{0}}{p_{\mathrm{BG}}}\right)^{0.775}
$$

gefunden. ${ }^{45}$ Bei einer endlichen Schlitzlänge von $1 \ll \frac{L}{D}<50$ und großen Abständen der normalen Stoßfront zum Düsenausgang $\frac{y_{\mathrm{M}}}{D}>\left(\frac{L}{D}\right)^{2 \cdot 0.47}$ sowie dem damit verbundenen relativ großen Druckverhältnis $\frac{p_{0}}{p_{\mathrm{BG}}}$ folgt die Position des frontalen Stoßes der 
Gleichung: ${ }^{25}$

$$
y_{\mathrm{M}}=0.67 \cdot D \cdot\left(\frac{p_{0}}{p_{\mathrm{BG}}}\right)^{0.5} \cdot\left(\frac{L}{D}\right)^{0.47}
$$

Für die beim Chilli- und Muesli-Jet auftretenden Schlitzdimensionen von $(0.5 \times 10) \mathrm{mm}^{2}$ wäre diese Formel somit nur für Werte von $x_{\mathrm{M}}>8 \mathrm{~mm}$ gültig, die jedoch bei vielen Chilli-Jet Messungen unterschritten werden.

Die Position der Kreuzung der lateralen Stoßfronten $x_{\mathrm{L}}$ bei einer Schlitzdüse endlicher Länge ergibt sich - ebenfalls empirisch - als: ${ }^{24}$

$$
y_{\mathrm{L}}=40 \cdot D \cdot\left(\frac{p_{0}}{p_{\mathrm{BG}}}\right)^{0.34}
$$

Insgesamt ist jedoch zu beachten, dass bereits geringe Abweichungen von der idealen Schlitzgeometrie zu deutlichen Einflüssen auf die Expansionsform selbst in größeren Abständen zur Düse führen können. ${ }^{37}$ Zudem hat sich gezeigt, dass bei Düsenabständen von mehr als einer Schlitzlänge häufig eine Beschreibung der Expansionen durch einen dreidimensionalen Fluss, wie er bei Lochdüsenexpansionen auftritt, realitätsnäher ist. ${ }^{28}$

Ein großer Anteil der spektroskopischen Untersuchungen an Überschallexpansionen beschäftigt sich mit der Region bis zur ersten normalen Stoßfront, da für die Spektrenaufnahme ja gerade die vorteilhaften Bedingungen der freien Expansion genutzt werden. ${ }^{24}$ Auch die darüber hinausgehenden Untersuchungen enden zumeist dicht hinter der Stoßfront. ${ }^{46}$ Dabei wird jedoch außer Acht gelassen, dass auch die Eigenschaften der Expansion jenseits davon von Interesse sein können, wenn sie denn ausreichend verstanden sind. Zahlreiche, zumeist nur die Dichtefluktuationen darstellende Bildgebungsverfahren haben hinter der ersten normalen Stoßfront eine Abfolge mehrerer Expansionen und Kompressionen gefunden (siehe z. B. Referenzen 25,44,47,48). Eine Darstellung einer solchen Sequenz aus einer Chilli-Jet-Expansion mit erhöhtem Hintergrunddruck sowie eine analoge Beobachtung eines Plasma-Jets aus einer Lochdüse ${ }^{44}$ sind in Abbildung $2.1 \mathrm{~d}$ und e gezeigt. Die sowohl - wie hier zu sehen - räumlich als auch zeitlich ${ }^{49,50,51}$ oszillierende Dichte entsteht dabei durch Reflexion von Stoßwellen an der Expansionsgrenze. ${ }^{48,52,53}$ Kapitel 6.3 befasst sich genauer mit den Dichte- und Temperaturfluktuationen in derartigen Expansionen.

\subsection{Eigenschaften von Multidüsenexpansionen}

Der Einsatz von Multidüsen für Untersuchungen in Überschallexpansionen gehört bislang nicht zu den gebräuchlichen Methoden. Der Begriff Multidüse soll hierbei eine Anordnung mehrerer Düsenöffnungen in einem Abstand zueinander, der eine geometrische Überlappung der getrennten Expansionen innerhalb des untersuchten Bereichs ermöglicht, bezeichnen. Numerische Berechnungen sagen für Multidüsenexpansionen das Auftreten komplexer Stoßwellenstrukturen voraus ${ }^{54}$, die bislang jedoch experimentell nicht hinreichend untersucht sind. 
Die Intentionen für eine Beschäftigung mit Multidüsenexpansionen sind ebenso breit gefächert wie ihre konkreten Konstruktionen. Bislang sind vorwiegend Multilochdüsenanordnungen bekannt. Der Einsatz mehrerer Düsenöffnungen kann dabei die gleichzeitige Expansion von Substanzen ermöglichen, die bei einer Vormischung miteinander reagieren würden. Mittels koaxialer, konzentrischer Lochdüsen, bei denen je eine Komponente durch die innere und die äußere Düse zugeführt wird, konnten derart reaktive Kombinationen bereits sowohl mit gepulsten ${ }^{55}$ als auch mit kontinuierlichen ${ }^{56}$ Expansionen erfolgreich untersucht werden.

Im Gegensatz zu Schlitzdüsen können Lochdüsen - wie in Abschnitt 2.2 geschildert - nicht unter Erhaltung der Expansionseigenschaften beliebig hochskaliert werden. Um dennoch längere Absorptionswege realisieren zu können, liegt eine lineare Anordnung mehrerer Lochdüsen nahe. Konkret wurde dieses Konzept von Herman et al. in der Form von 31 Lochdüsen $(d=400 \mu \mathrm{m})$ im Abstand von $5 \mathrm{~mm}$ zueinander angewendet. ${ }^{24,57}$ Dabei gingen sie zwar von einer gegenseitigen Beeinflussung nebeneinanderliegender Expansionen sowie der Bildung eines komplexen Stoßwellenmusters aus ${ }^{24}$, aufgrund der Begrenzung der Expansionen durch seitliche Stoßfronten jedoch nicht von einer Kreuzung der freien Expansionen. ${ }^{57}$ Es wurde eine deutliche Verformung und Einengung der Zonen freier Expansion gegenüber den bekannten Mustern aus Einzeldüsen aufgrund der kollidierenden seitlichen Stoßfronten beobachtet. Die Temperaturanalyse dieser Multilochdüsenexpansionen unter Einsatz von $\mathrm{N}_{2} \mathrm{O}$ zeigte das Vorliegen zweier verschiedener Rotationstemperaturen von 44 und $211 \mathrm{~K}$, die jeweils den Zonen freier Expansion und ihren Zwischenräumen zugeordnet wurden ${ }^{24}$ und somit die These der Existenz von Kollisionszonen zwischen den Einzelexpansionen bestätigten.

Eine Verwendung von Multischlitzdüsenaufbauten zur reinen Erhöhung des Absorptionsweges ist aufgrund der unproblematischen Verlängerung von einfachen Schlitzdüsen nicht sinnvoll. Allerdings kann die Kombination aus - im Vergleich zu Lochdüsen - verstärkter Aggregation ${ }^{24}$ und der großen Anzahl von Kollisionszonen genutzt werden, um warme Expansionen mit entsprechend wärmeren Clustern ${ }^{58}$ und energetisch höherliegenden Molekülkonformeren zu erzeugen. ${ }^{59,60}$ Mit dem Originalaufbau des Muesli-Jets (siehe Abschnitt 3.2), der entweder mit 20 Schlitzen $\left(10 \times 0.5 \mathrm{~mm}^{2}\right)$ im Abstand von $5 \mathrm{~mm}$ oder mit 10 Schlitzen im Abstand von $10 \mathrm{~mm}$ betrieben werden kann, sollte der Einfluss des Abstandes zwischen parallelen Schlitzdüsen untersucht werden. Hierbei konnte bereits die stärkere Erwärmung von Monomeren und Aggregaten bei Expansionen durch nah beieinanderliegende, parallele Schlitzdüsen relativ zu weiter voneinander entfernten Schlitzen und Einzelschlitzdüsen festgestellt werden. ${ }^{59,60}$

Weiterhin wurde bei den Schlitzdüsenexperimenten auch der Ansatz der synchronen Expansion zweier getrennter Komponenten durch abwechselnde Zufuhr zu den 20 Düsenöffnungen verfolgt. Im Gegensatz zu den Multilochdüsenexperimenten ${ }^{57}$ wurde hier von einer ausreichenden Überlappung nebeneinanderliegender Expansionen für die Generierung gemischter Aggregate ausgegangen. Diese konnten für das System 2,2,2-Trifluorethanol und Wasser (jeweils in Helium) auch nachgewiesen werden ${ }^{60}$, jedoch im Rahmen dieser Arbeit für die Kombination Methanol und tert-Butylalkohol 
nicht reproduziert werden. Die Ursachen für diese Diskrepanz werden in Abschnitt 3.2.3 diskutiert.

\subsection{Chemische Bildgebung von Überschallexpansionen}

Die Bildgebung von Überschallexpansionen beschränkt sich häufig auf die Analyse physikalischer Größen wie der Dichte, Strömungsgeschwindigkeit oder Temperatur. Diese können entweder wie bei der Druckmessung mithilfe eines Pitotrohrs ${ }^{37,43,47,61}$ direkt gemessen oder mittels optischer Methoden indirekt untersucht werden. Häufig werden optische Dichtegradienten mittels Schlieren-Fotographie sichtbar gemacht ${ }^{49,51,62,63,64,65,66}$, wobei sogar eine zeitaufgelöste Visualisierung möglich ist. ${ }^{49,51,62}$ Änderungen des Brechungsindexes können durch Unterschiede in der Lichtstreuung ${ }^{49,50}$ sowie interferometrisch sichtbar gemacht werden ${ }^{48,66,67}$, wobei tomographische Darstellungen ${ }^{67,68}$ sogar dreidimensionale Einblicke in Überschallexpansionen ermöglichen. Im Falle Licht emittierender Systeme wie Plasma-Jets ${ }^{44}$ kann zudem Fotographie Informationen über Expansionsstrukturen und -eigenschaften liefern. Weiterhin hilfreich für die Untersuchung von Überschallexpansionen sind die akustischen Phänomene. Sowohl die turbulente Ausbreitung der Expansion im Hintergrundmedium als auch die Wechselwirkung verschiedener Strukturelemente der Expansion mit den periodischen Stoßzellen (siehe Abschnitt 2.3) können die Emission akustischer Signale bewirken, die sich in Abhängigkeit von ihrer Ursache in ihrem Frequenzspektrum und ihrer Ausbreitungsrichtung unterscheiden. ${ }^{52,69}$ Somit können räumlich variabel platzierte Mikrophone zur Aufklärung der Expansionsstruktur beitragen. ${ }^{51,61,63,64,65,69}$

Das Methodenspektrum zur Analyse von Überschallexpansionen wird jedoch enger, wenn zugleich spektroskopische Informationen über die Substanzen innerhalb der Expansion gewonnen werden sollen. Hier finden zumeist Mapping-Techniken Anwendung, bei denen ein Raster von Spektren, die jeweils nur einen kleinen räumlichen Bereich erfassen, aufgenommen und anschließend zu einem Gesamtbild zusammengefügt wird. Hierbei müssen entweder die Probe / Expansionsdüse ${ }^{70,71}$, der Detektor ${ }^{72}$ oder beide ${ }^{47}$ im Raum bewegt werden. Da immer nur ein geringer Ortsausschnitt gleichzeitig erfasst werden darf, eignen sich besonders laserbasierte Methoden für den Mapping-Einsatz. Hierbei gibt es sowohl Beispiele unter Verwendung von Absorptionsspektroskopie ${ }^{71}$ als auch von laserinduzierter Fluoreszenz. ${ }^{24,47,73} \mathrm{Um}$ schwingungsspektroskopische Einblicke zu erhalten, ist besonders das Raman-Mapping gut geeignet, da hier sehr hohe räumliche Auflösungen von bis zu $2 \mu \mathrm{m}$ erreicht werden können ${ }^{74}$ und die gewonnenen Spektren Informationen über Aggregatstrukturen, Temperaturen, Dichte und Geschwindigkeiten enthalten. ${ }^{46,70,74,75,76,77,78}$ Da aufgrund der hohen Anzahl aufgenommener Einzelspektren die Messzeiten lang sind ${ }^{70}$ und somit auch der Substanzverbrauch hoch ist, werden für großflächigere Untersuchungen wie die von Stoßwellenstrukturen häufig nur Spektren an weit auseinanderliegenden Punkten oder nur entlang einzelner Linien gemessen. ${ }^{77,78}$ Eine ortsaufgelöste schwingungsspektroskopische Untersu- 
chung von Überschallexpansionen mittels FTIR-Spektroskopie erfordert aufgrund des großen Strahldurchmessers den Einsatz von Aperturen, wodurch entweder die Nachteile einer signifikanten Lichtabschwächung toleriert ${ }^{10}$ oder intensive Lichtquellen wie Synchrotronstrahlung aus Elektronenspeicherringen ${ }^{79}$ oder Freie-Elektronen-Lasern ${ }^{80}$ eingesetzt werden müssen. Zusätzlich entstehen Probleme durch Beugung an den Aperturkanten, so dass die erreichbare räumliche Auflösung begrenzt ist. ${ }^{81}$

Eine Alternative zu Infrarot-Mapping-Methoden stellt der Einsatz von FTIR-Spektroskopie in Verbindung mit Focal Plane Array (FPA) Detektoren dar. Dies sind Infrarotdetektoren, die sich aus tausenden von Einzelelementdetektoren mit jeweils separatem Ausleseschaltkreis zusammensetzen. ${ }^{82}$ Sie ermöglichen eine synchrone, ortsaufgelöste Aufnahme von entsprechend vielen Infrarotspektren, so dass mit jedem Scan die komplette untersuchte Fläche der Expansion spektral erfasst werden kann. Dies bietet Vorteile im Bezug auf die Gesamtmesszeit, den Substanzverbrauch, den Lichtdurchsatz und die Reproduzierbarkeit. FPA-Detektoren werden zumeist in der Infrarotmikroskopie zur Untersuchung von biomedizinischen Proben ${ }^{81,83,84}$ oder unter materialwissenschaftlichen Aspekten ${ }^{85}$ verwendet und können sowohl für Messungen der Transmission, Reflexion oder abgeschwächten Totalreflexion (ATR) eingesetzt werden. ${ }^{81}$ Dabei werden die FTIR-Spektren zumeist im Step-Scan-Modus aufgenommen ${ }^{81,83,85,86}$, da hier die Anforderungen an die Datenauslesegeschwindigkeit im Vergleich zum Rapid-ScanModus $^{87,88}$ geringer sind. Die in teilweise nur Sekunden aufgenommenen großen Datenmengen bei FPA-Messungen liefern dabei sowohl breitbandige spektrale als auch räumliche Informationen über die untersuchte Probe oder Expansion, stellen gleichzeitig jedoch auch eine Herausforderung an die eingesetzte Soft- und Hardware dar. ${ }^{38,81}$ Eine umfassende Übersicht über die allgemeine Funktionsweise von FPA-Detektoren sowie ihre Aufbau-, Elektronik- und Materialvarianten kann der Literatur ${ }^{82,89,90,91}$ entnommen werden.

Der Einsatz von Focal Plane Array Detektoren birgt jedoch gegenüber Einzelelementdetektoren auch Nachteile, insbesondere bezüglich des Signal-zu-Rauschen-Verhältnisses der erhaltenen Spektren. Daher werden sie bislang vorwiegend für Proben mit starken Absorbanzen eingesetzt, die Kombination mit den verdünnten Gasphasensystemen der Überschallexpansionen ist jedoch neuartig. Das verhältnismäßig hohe Rauschlevel von FPA-Spektren ist teilweise auf Effekte zurückzuführen, die auch bei Einzelelementdetektoren auftreten, dort jedoch wegen der größeren Detektorfläche weniger stark ins Gewicht fallen. Aufgrund der geringen Einzelflächen der FPA-Detektorelemente ist auch die einfallende Menge an Photonen pro Detektor gering. Die Signalintensität kann sich somit innerhalb der Größenordnung der geringsten detektierbaren Spannungsänderung des Detektorelements bewegen und liegt daher zwingend in der Größenordnung des Rauschens (least significant bit noise). ${ }^{92}$ Dies kann durch den Einsatz intensiverer Lichtquellen wie Synchrotronstrahlung verbessert werden, was jedoch die Größe der simultan untersuchbaren Fläche einschränkt ${ }^{83}$ und zur Detektorübersättigung führen kann ${ }^{82}$. Die hohe Pixelanzahl bewirkt zusätzlich, dass prinzipiell selten auftretende Rausch-Spitzen (noise spikes) im Interferogramm bei einem signifikanten 
Anteil der Scans auftreten und somit sinusförmige Störungen im Spektrum verursachen, die auch durch eine Spektrenmittelung nicht hinreichend reduziert werden können. ${ }^{38,93}$ Im Rapid-Scan-Modus können sie jedoch durch den Einsatz von Hochpassfiltern unterdrückt werden. ${ }^{38}$

Zusätzlich existieren für FPA-Detektoren auch Rauschquellen, die neben den kleinen Detektorflächen auch auf die sehr kompakte Auslese- und Verarbeitungselektronik sowie auf die komplexen elektrischen Grenzflächen zurückzuführen sind. ${ }^{93}$ Unzulänglichkeiten in der Separierung der einzelnen Ausleseschaltkreise können eine Signalübertragung zwischen Pixeln (crosstalk) hervorrufen. ${ }^{82}$ Zudem führen schon geringfügige Unterschiede in der Leistungsfähigkeit der Einzeldetektoren zu einem allgemeinen Bildrauschen (spatial / fixed pattern noise), das, wenn es zeitlich nicht vollständig konstant ist, nicht durch eine Kalibrierung kompensierbar ist. ${ }^{38,86}$ Einen ähnlichen Effekt hat eine ungleichmäßige Ausleuchtung der Einzeldetektoren. ${ }^{86,92}$ Die komprimierte Elektronik kann zudem häufig nur einen unvollständigen Übertrag des gemessenen Signals gewährleisten und verringert so zusätzlich das Signal-zu-Rauschen-Verhältnis. ${ }^{82}$ Auch weisen FPA-Detektoren eine hohe Empfindlichkeit auf Temperaturschwankungen auf, die Einfluss auf die Spektrenqualität haben kann. ${ }^{94}$ Weitere, teilweise durch Kalibrierungen und Mittelungen nicht kompensierbare Rauschintensität und Störungen im Spektrum können durch Fehler in der Datenbündelung innerhalb der Ausleseschaltkreise entstehen.

Eine Verbesserung des FPA-Rauschlevels ist im Wesentlichen auf drei Arten möglich. ${ }^{92}$ Hardwarekomponenten wie Bandpassfilter oder Diffusoren können zur Steigerung des Signals im relevanten Spektralbereich und zur Homogenisierung der Ausleuchtung eingesetzt werden. ${ }^{86,92}$ Verbesserungen in der Datenaufnahme können durch eine Verlängerung der Integrationszeit oder Mittelung mehrerer Scans erhalten werden. Außerdem stehen Methoden zur Datennachbehandlung wie die Zusammenfassung mehrerer Pixel (binning), Hintergrundsubtraktion, Offset-Korrekturen ${ }^{94}$ und weitere individuell problemorientierte, mathematische Korrekturverfahren zur Verfügung. ${ }^{92}$ Die häufig geringe Signalintensität kann durch eine Schaltverstärkung (gain ranging) verbessert werden. ${ }^{92}$

Insgesamt ermöglicht die chemische Bildgebung von Überschallexpansionen durch den Einsatz von FTIR-Spektroskopie in Verbindung mit Focal Plane Array Detektoren also eine synchrone Aufnahme tausender ortsaufgelöster Infrarotspektren und bietet deutliche Vorteile gegenüber Mapping-Methoden im Hinblick auf Messzeit, Substanzverbrauch, Reproduzierbarkeit und zum Teil der Breite des erfassten Spektralbereichs. Der Informationsgehalt der so gewonnenen Spektren erstreckt sich gleichzeitig über Daten zur Dichte, Temperatur, Aggregations- und Konformationsdynamik in verschiedenen Zonen der Expansionen. Die Technik birgt aufgrund ihrer Komplexität auf geringem Raum jedoch noch einige Nachteile im Bezug auf spektrales Rauschen, Bildrauschen und Artefakte, die sich jedoch für folgende Detektorgenerationen voraussichtlich reduzieren werden. 


\subsection{Temperaturabschätzung in Überschallexpansionen}

Temperaturen innerhalb von Überschallexpansionen sind prinzipiell aus den Stagnationsbedingungen des Gases (gekennzeichnet durch den Index 0) sowie aus den Strömungseigenschaften der Expansion für die verschiedenen Molekülfreiheitsgrade berechenbar. Eine derartige Analyse wurde beispielsweise von DePaul et al. für geringe Abstände und trägergasreiche Expansionen durch Lochdüsen durchgeführt. ${ }^{29}$ Dabei wurde von der Annahme ausgegangen, dass die Expansion zunächst als Kontinuumsströmung beschrieben werden kann, in der die Temperatur für alle Komponenten und Freiheitsgrade aufgrund der hohen Stoßrate identisch ist. Daher kann die Temperatur für jeden Punkt der Expansion aus der Machzahl, dem Poisson-Koeffizienten und der Stagnationstemperatur berechnet werden. Die im späteren Verlauf der Expansion auftretenden Unterschiede für die einzelnen Freiheitsgrade werden dabei als nur abhängig von dem Abstand zur Düse betrachtet, an dem sie aufgrund der abnehmenden Stoßzahl nicht mehr an der Energieumverteilung innerhalb der Expansion beteiligt sind (sudden freeze model). ${ }^{29}$ Die so erreichte Endtemperatur für jeden Freiheitsgrad kann dann anhand der Stagnationsbedingungen, der Düsengeometrie, der stofflichen Zusammensetzung der Expansion, der Stoßquerschnitte der Moleküle und deren Abhängigkeit von der Stoßenergie sowie aus dem „sudden freeze“-Modell stammenden Parametern bestimmt werden. ${ }^{29}$

Aufgrund der erforderlichen Kenntnis zahlreicher Größen und der hohen Anzahl enthaltener Näherungen erfolgt eine Temperaturvorhersage für Überschallexpansionen häufig (semi-)empirisch. ${ }^{25,29}$ Für Expansionen aus Schlitzdüsen wurden in Abhängigkeit vom Abstand zur Düse beispielsweise folgende Zusammenhänge zwischen der (Rotations-) Temperatur $T$ und dem Abstand $y$ gefunden: ${ }^{25}$

$$
\begin{array}{ll}
\frac{T}{T_{0}}=0.53\left(\frac{y}{D}\right)^{1-\gamma} & \text { für } \frac{y}{D}<\frac{L}{D} \\
\frac{T}{T_{0}}=0.53\left(\frac{L}{D}\right)^{\gamma-1}\left(\frac{y}{D}\right)^{2(1-\gamma)} & \text { für } \frac{L}{D}<\frac{y}{D}<\frac{y_{\mathrm{M}}}{D}
\end{array}
$$

mit $L$ : Düsenlänge, $D$ : Düsenbreite, $\gamma$ : Poisson-Koeffizient, $y_{\mathrm{M}}$ : Position der ersten normalen Stoßfront.

Liegen ortsaufgelöste spektrale Informationen zu der betrachteten Expansion vor, ermöglichen diese ebenfalls die Temperaturbestimmung für die verschiedenen Freiheitsgrade. Betrachtet werden sollen hier schwingungsspektroskopisch untersuchte Expansionen von Substanzen mit infrarotaktiven Schwingungsmoden mit oder ohne Einsatz eines zusätzlichen Trägergases.

Eine Bestimmung der Translationstemperatur $T_{\mathrm{t}}$ ist grundsätzlich möglich, wenn geeignete vereinfachende Annahmen getroffen werden und genügend Fluss- und Stoffparameter für die Expansion bekannt sind. Im einfachsten Fall wird angenommen, dass die Translationstemperatur ausschließlich an den (experimentell leicht zugänglichen) 
Dichteabfall eines idealen Gases in einer isentropen Expansion gekoppelt ist. Unter diesen Bedingungen ergibt sich die Translationstemperatur als: ${ }^{24,28,95}$

$$
\frac{T_{\mathrm{t}}}{T_{0}}=\left(\frac{p}{p_{0}}\right)^{\frac{\gamma-1}{\gamma}}=\left(\frac{\rho}{\rho_{0}}\right)^{\gamma-1}
$$

mit $p$ : Druck, $\rho$ : Dichte, $\gamma$ : Poisson-Koeffizient, Index 0: Stagnationsbedingung.

Werden die realen Eigenschaften von Gasen mit in die Berechnung einbezogen, sind zusätzliche Informationen über den genauen Verlauf der Teilchenzahldichte sowie Transportkoeffizienten und thermodynamische Eigenschaften des expandierten Gases notwendig. ${ }^{76}$ Alternativ kann die Translationstemperatur auch aufgrund der Annahmen von Isentropie der Expansion und Energieerhalt unter Vernachlässigung einer Anregung von Schwingungsfreiheitsgraden aus den Besetzungen von Rotationsniveaus und dem Dichteabfall der expandierten Substanz berechnet werden. ${ }^{96}$

Rotationstemperaturen werden häufig aufgrund einer Intensitätsanalyse einzelner Rotationslinien eines Schwingungsübergangs bestimmt. ${ }^{24,47,71,73,96,97}$ Sie basieren zumeist auf der Annahme einer Boltzmannverteilung der Besetzung der Energieniveaus. Zusammen mit der $(2 J+1)$ fachen Entartung der Rotationsniveaus ergibt sich so für die Anzahl von Molekülen im Rotationsniveau $J$ eines Schwingungsgrundzustandes: ${ }^{98}$

$$
N_{J} \propto(2 J+1) \exp \left(-\frac{F(J) h c}{k T}\right)
$$

mit dem Rotationsenergieterm $F$, dem Planckschen Wirkungsquantum $h$, der Lichtgeschwindigkeit $c$ und der Boltzmannkonstante $k$.

Im Grenzfall eines starren Rotators gilt für die Rotationsenergie $F$ :

$$
F(J)=B J(J+1)
$$

mit der Rotationskonstanten $B$.

Für das Intensitätsverhältnis zweier Linien der Übergänge $J_{1}^{\prime} \leftarrow J_{1}^{\prime \prime}$ und $J_{2}^{\prime} \leftarrow J_{2}^{\prime \prime}$ einer Schwingungsfundamentalen eines linearen Moleküls gilt näherungsweise: ${ }^{98}$

$$
\frac{I_{1}}{I_{2}}=\frac{\tilde{\nu}_{1}}{\tilde{\nu}_{2}} \cdot \frac{J_{1}^{\prime}+J_{1}^{\prime \prime}+1}{J_{2}^{\prime}+J_{2}^{\prime \prime}+1} \cdot \frac{\exp \left(-\frac{B_{1}^{\prime \prime} J_{1}^{\prime \prime}\left(J_{1}^{\prime \prime}+1\right) h c}{k T_{\mathrm{rot}}}\right)}{\exp \left(-\frac{B_{2}^{\prime \prime} J_{2}^{\prime \prime}\left(J_{2}^{\prime \prime}+1\right) h c}{k T_{\mathrm{rot}}}\right)}
$$

Sind also aus den gemessenen Spektren die Wellenzahlen $\tilde{\nu}$ und die Bandenintensitäten $I$ sowie die Zuordnungen der einzelnen Übergänge und die Rotationskonstanten bekannt, können durch diesen Zusammenhang Rotationstemperaturen $T_{\text {rot }}$ berechnet werden. Dies ist aus einzelnen Wertepaaren möglich, liegt jedoch eine höhere Zahl auswertbarer Banden vor, so ist die Temperaturbestimmung aus geeigneten Auftragungen die präzisere Methode. ${ }^{24,43,71,99}$ Sie ermöglicht sogar die Analyse verschiedener Temperaturkomponenten im gemessenen Probevolumen. ${ }^{24,43}$ Bei mehratomigen Molekülen 
und komplexen Bandenmustern können zusätzlich Simulationsprogramme und Spektrendatenbanken die Bandenzuordnung und Temperaturanalyse unterstützen. ${ }^{43}$

Ist die spektrale Auflösung für eine Separierung der einzelnen Rotationslinien nicht ausreichend, kann dennoch eine Abschätzung der Rotationstemperatur erfolgen. Ist eine deutliche Unterscheidung zwischen P- und R-Zweig einer Bande möglich, kann die Rotationstemperatur aus der Wellenzahldifferenz der Intensitätsmaxima beider Zweige bestimmt werden. Die Methode basiert auf den formal nur für zweiatomige Moleküle gültigen Gleichungen 2.6 und 2.7. Für das Intensitätsmaximum von $J$ gilt näherungsweise $\left.\frac{\mathrm{d} N_{J}}{\mathrm{~d} J}\right|_{J_{\max }}=0$. Hieraus folgt für $J_{\max }:{ }^{98}$

$$
\begin{aligned}
J_{\max } & =\sqrt{\frac{k T_{\text {rot }}}{2 h c B}}-\frac{1}{2} \\
\operatorname{Mit} \quad \tilde{\nu} & =\tilde{\nu}_{0}+F^{\prime}(J)-F^{\prime \prime}(J)
\end{aligned}
$$

mit $\tilde{\nu}_{0}$ als Wellenzahl des reinen Schwingungsübergangs ${ }^{100}$ ergibt sich so als Wellenzahldifferenz zwischen den beiden Zweigen: ${ }^{98}$

$$
\Delta \tilde{\nu}_{\mathrm{PR}}^{\max }=\sqrt{\frac{8 k T_{\mathrm{rot}} B}{h c}} \propto \sqrt{T_{\mathrm{rot}}}
$$

Während für lineare mehratomige Moleküle die Bestimmung aus der Rotationskonstanten zu deutlichen Abweichungen von der Realität führen kann, ist bei Vorliegen eines Spektrums bei bekannter Temperatur (z. B. Gasphasenspektrum bei Zimmertemperatur) eine Kalibrierung möglich, so dass für die eigentliche Auswertung nur der Proportionalitätszusammenhang zwischen Wellenzahldifferenz und Wurzel der Rotationstemperatur genutzt wird. Ist bei einer Bande weder die Rotations- noch die grobe Zweigstruktur erkennbar, so kann dennoch eine qualitative Analyse der Halbwertsbreite erfolgen, da sich aufgrund der stärkeren Besetzung von angeregten Rotationszuständen bei höheren Temperaturen die Rotationsschwingungsbanden verbreitern.

Schwingungstemperaturen können anhand der Bandenintensitäten einer Fundamentalen und einer zugehörigen heißen Bande bei Kenntnis der Übergangsmomente der betrachteten Schwingungen ermittelt werden. ${ }^{70,75}$ Wie die meisten Techniken zur Bestimmung von Rotationstemperaturen basiert auch diese Methode auf der Annahme einer Zustandsbesetzung gemäß Boltzmannverteilung. Alternativ kann auch eine Auswertung der Bandenform eines Schwingungs-Q-Zweigs im Vergleich zu gleichartigen Spektren bekannter Temperaturen erfolgen, da der Q-Zweig kaum durch Rotationsanregungen beeinflusst wird. ${ }^{95}$

Werden Schwingungsspektren von Molekülaggregaten untersucht, so kann auch eine Veränderung der Clustergrößenverteilung als relatives Temperaturmaß verwendet werden, da verschiedene Cluster abhängig von ihrer Bindungsenergie unterschiedlich leicht dissoziieren. ${ }^{101}$ Wasserstoffbrückengebundene Systeme weisen gegenüber anderen Molekülaggregaten zusätzlich noch den Vorteil der stärkeren Temperaturabhängigkeit von 
Bandenpositionen und Halbwertsbreiten auf ${ }^{1,11,102}$, die für relative Temperaturabschätzungen genutzt werden können.

Problematisch für viele dieser Methoden ist die zugrunde liegende Annahme von Boltzmannverteilungen, die in den Nicht-Gleichgewichtssystemen der Überschallstrahlen eigentlich nicht anwendbar sind. Zusätzlich ist häufig zwar eine sinnvolle Temperaturabschätzung für strukturell gut aufgelöste Banden von Monomeren möglich, diese lässt sich jedoch nicht auf die zugehörigen Aggregate übertragen. Aufgrund der Kondensationswärme bei der Aggregation, die im Jet nicht vollständig an ein Badgas abgeführt werden kann, liegen die Clustertemperaturen üblicherweise über denen der Monomere. Gleichzeitig tritt jedoch auch der gegenteilige Effekt der evaporativen Kühlung von Clustern beim Abdampfen von hoch angeregten Monomeren auf. ${ }^{78}$ Selbst unterschiedliche Kernspinisomere des gleichen Moleküls können im Jet unterschiedliche Temperaturen aufweisen. ${ }^{103}$ Temperaturabschätzungen in Überschallstrahlen müssen daher grundsätzlich unter Vorbehalt gesehen und auf ihre Plausibilität geprüft werden. 


\section{Experimentelles}

\subsection{Genereller Aufbau der Jet-Apparaturen}

Da die verschiedenen verwendeten Apparaturen (Muesli-Jet, Popcorn-Jet, Chilli-Jet) für die FTIR-Spektroskopie am Überschallstrahl auf dem gleichen grundsätzlichen Aufbauprinzip basieren, soll dieses hier zunächst separat dargestellt werden. Abweichungen von diesem allgemeinen Schema sowie genauere Spezifikationen der verwendeten Komponenten werden im Anschluss für die einzelnen Aufbauten in den Abschnitten 3.2, 3.3 und 3.4 präzisiert. Details zu den eingesetzten Chemikalien befinden sich im Anhang, Abschnitt A.2.

\subsection{1 Überschallexpansion}

Im Fall der Expansionen mit einem Trägergas wurde aus den in Abschnitt 2.2 aufgeführten Gründen Helium gewählt. Die Mischung mit dem gasförmigen $\mathrm{N}_{2} \mathrm{O}$ als Probesubstanz wurde durch die Vormischung in einer Druckgasflasche in der gewünschten Konzentration erreicht und das erhaltene Gemisch direkt ins Reservoir geleitet. Die leichtflüchtigen festen (tert-Butylalkohol) und flüssigen (Methanol, Wasser) Substanzen wurden in einen temperierbaren Sättiger gefüllt, in dem sie vom Helium um- bzw. durchströmt wurden. Die vom Trägergas aufgenommene Substanzmenge ist hierbei einerseits über die Sättigertemperatur und somit den Dampfdruck und andererseits über eine zusätzliche Zumischung von Helium variierbar. Diese Zumischung kann nötig werden, da die zur Substanzkühlung verwendeten Kryostaten in ihrer Minimaltemperatur auf etwa $-30{ }^{\circ} \mathrm{C}$ beschränkt sind. Ebenso kann im Sättiger maximal eine Temperatur etwa $3{ }^{\circ} \mathrm{C}$ unterhalb der Umgebungstemperatur gewählt werden, da andernfalls eine unerwünschte Kondensation innerhalb des Reservoirs oder der Zuleitungen erfolgt. Aus diesem Grund wurde bei Stoffen mit niedrigem Dampfdruck (Imidazol, Phenylimidazol) der Popcorn-Jet mit seiner separaten Heizmöglichkeit verwendet. Über die Partialdrücke der untersuchten Substanzen im Gasgemisch sowie über den Gesamtdruck im Reservoir können sowohl die Größe der gebildeten Cluster als auch das Ausmaß der Kühlung in der Expansion variiert werden.

Um während der Expansionen einen möglichst konstanten Gasstrom aufrecht erhalten zu können, werden die Helium-Probe-Gasmischungen zunächst über Magnetventile in Reservoire mit über $60 \mathrm{~L}$ Fassungsvermögen geleitet. Ein konstanter Druck im Reservoir wird dabei über die Kopplung der Magnetventilöffnung an die Druckmessung 
innerhalb des Reservoirs mit einem voreingestellten Solldruck gewährleistet. Um eine Minimierung des Druckabfalls während der Expansion zu erreichen, wird der HeliumVordruck jeweils deutlich höher gewählt als der Reservoirdruck. Die Mischung mehrerer Komponenten innerhalb eines Reservoirs wird über bis zu drei Reservoirzugänge und die Einstellung der jeweiligen Öffnungsintervalle der Magnetventile für jeden Zugang realisiert.

Da für die meisten Messungen gepulste Expansionen verwendet werden, wird der Gasfluss vom Reservoir zur Düse über mit den Spektrometerscans synchronisierte Magnetventile geregelt. Um während der Gaspulse einen niedrigen Hintergrunddruck aufrecht erhalten zu können, strömt das Gas in mehrere Kubikmeter große Puffervolumina, die mithilfe von Pumpensystemen kontinuierlich evakuiert werden. Die Pause zwischen zwei Gaspulsen entspricht dabei der Zeit, die für das Evakuieren des Pufferraums auf etwa ein Hundertstel des Maximaldrucks während des Gaspulses benötigt wird.

\subsubsection{Spektrenaufnahme und Synchronisation}

Die Aufnahme von FTIR-Spektren erfolgt mit Spektrometern der Firma Bruker und der zugehörigen OPUS-Software (Versionen 6.0, 6.5 und 7.0). Da der Schwerpunkt dieser Arbeit auf der Analyse der $\mathrm{O}-\mathrm{H}-$ und N-H-Fundamentalstreckschwingungen liegt, wurden Messungen im mittleren Infrarotbereich $\left(4000-400 \mathrm{~cm}^{-1}\right)$ durchgeführt. Daher wurden als Breitband-Lichtquellen Siliziumcarbid-Keramiken (Globars) verwendet, deren Licht im Interferometer mit beschichteten Kaliumbromid-Strahlteilern moduliert wurde. Der aus dem Spektrometer austretende Infrarotstrahl wird durch Linsen auf einen Bereich dicht hinter der Düse fokussiert, da hier die Teilchendichten innerhalb der Expansion noch recht hoch sind und so die Absorbanz maximiert werden kann. Die Fokussierung erfolgt, um die Heterogenität des gemessenen Bereichs zu verringern. Auch im Fokus beträgt der Strahldurchmesser noch mehrere Millimeter, so dass alle gezeigten Spektren immer eine Mittelung über eine größere Expansionszone darstellen. Nach dem Passieren der Expansion wird der Lichtstrahl über einen Parabolspiegel auf einen MCT- oder InSb-Detektor fokussiert. Der Einsatz eines spektralen Filters vor dem Detektor ermöglicht die Verwendung einer größeren Apertur innerhalb des Spektrometers und somit eine höhere Photonenzahl im für die Messung relevanten Spektralbereich und eine entsprechende Erhöhung des Signal-zu-Rauschen-Verhältnisses.

Die Synchronisation von Spektrometerscan und Gaspuls erfolgt über ein TTL-Signal, das vor dem Probenscan vom Spektrometer an einen Pulsgenerator, der die Magnetventile an der Düse steuert, weitergegeben wird. Mithilfe manuell eingestellter Öffnungsdauer- und Verzögerungszeiten kann die zeitliche Abstimmung von Scan und Puls empirisch optimiert werden. Hierbei erfolgt die Ventilöffnung zeitlich so weit vor Scanbeginn, dass bis zur Messung stabile Expansionsbedingungen erreicht werden. Das Schließen sollte soweit nach Ende der Probenmessung geschehen, dass diese nicht durch den Schließprozess beeinträchtigt wird, gleichzeitig ist jedoch auch eine Minimierung 
des Gasverbrauchs anzustreben. Die optimierten Öffnungsparameter für die verschiedenen Apparaturen und Messmodi können Kapitel A.6.2 im Anhang entnommen werden.

Der Messablauf wird über eine trs- (time resolved spectroscopy) Methode gesteuert. Diese erlaubt die sequentielle Messung mehrerer Spektrometerscans in definierten zeitlichen Abständen. Ein typischer Messablauf setzt sich dabei aus der Aufnahme von 20 Hintergrundscans, 2 Vorscans, 1 Probenscan und $3 \times 1$ Nachscans zusammen. Die hohe Anzahl von Hintergrundscans dient der Rauschminimierung in den später erhaltenen Spektren. Sie werden vor jedem Probenscan aufgenommen, um die zeitliche Distanz und somit auch die Änderungen der Umgebungsverhältnisse zwischen Hintergrundund Probenspektrum gering zu halten. In den seltenen Fällen, wo sich selbst diese Zeitdifferenz als zu groß herausstellt, können alternativ die Vorscans als Hintergrund verwendet werden. Neben dem Nachteil ihrer geringeren Anzahl können sie auch durch elektronische Störungen aufgrund der Öffnung der Düsen-Magnetventile für den darauffolgenden Probenscan beeinträchtigt sein. Der Probenscan stellt den eigentlichen Messvorgang dar und ist wie oben erläutert mit den gepulsten Expansionen synchronisiert. Im Anschluss an die Expansion werden noch zwei bis drei einzelne Nachscans gemessen. Diese nehmen die Gasphasenspektren des gleichzeitig abgepumpten Expansionsgases auf und können so zur Differenzierung von Monomer- und Clusterbanden beitragen. Für jeden Puls wird die gesamte Scan-Sequenz innerhalb einer Multi-Spektren-Datei abgespeichert. Der genaue Messablauf erfolgt makrogesteuert. Die unterschiedlichen Messmakros sind in Abschnitt A.7 zu finden, die genaue Scan-Abfolge innerhalb der typischen verwendeten trs-Methode kann Abschnitt A.6.1 entnommen werden.

\subsubsection{Datenverarbeitung}

Während der Evakuierpausen zwischen den Gaspulsen werden die einzelnen Spektren aus der Multi-Spektren-Datei extrahiert und entsprechend ihrem Typ (Hintergrund, Vorscan, Probenscan, Nach1/2/3-Scan) mit verschiedenen Dateiendungen abgespeichert. Nach Beendigung aller Scansequenzen der Messreihe werden Spektren gleichen Typs gemittelt und die erhaltenen Spektren Fourier-transformiert. Die Reihenfolge von Mittelung und Fourier-Transformation kann bei Messungen, in denen wiederholt Störungen auftreten, vertauscht werden. Dies erleichtert die Identifizierung der „Ausreißerspektren“, ist jedoch zeitaufwändiger. Die erhaltenen Einkanalspektren von Probeund Nachscans werden im Anschluss jeweils durch das gemittelte Hintergrundspektrum dividiert und die resultierenden Transmissions- in Absorbanzspektren umgewandelt.

Wie die Messung erfolgt auch die anschließende Datennachbehandlung makrogestützt. Die einzelnen Auswertemakros sind Abschnitt A.7 zu entnehmen, die bei den verschiedenen Spektren verwendeten Parameter für die Fourier-Transformation befinden sich in den jeweiligen Parameterblöcken der Spektren in Abschnitt A.5. 


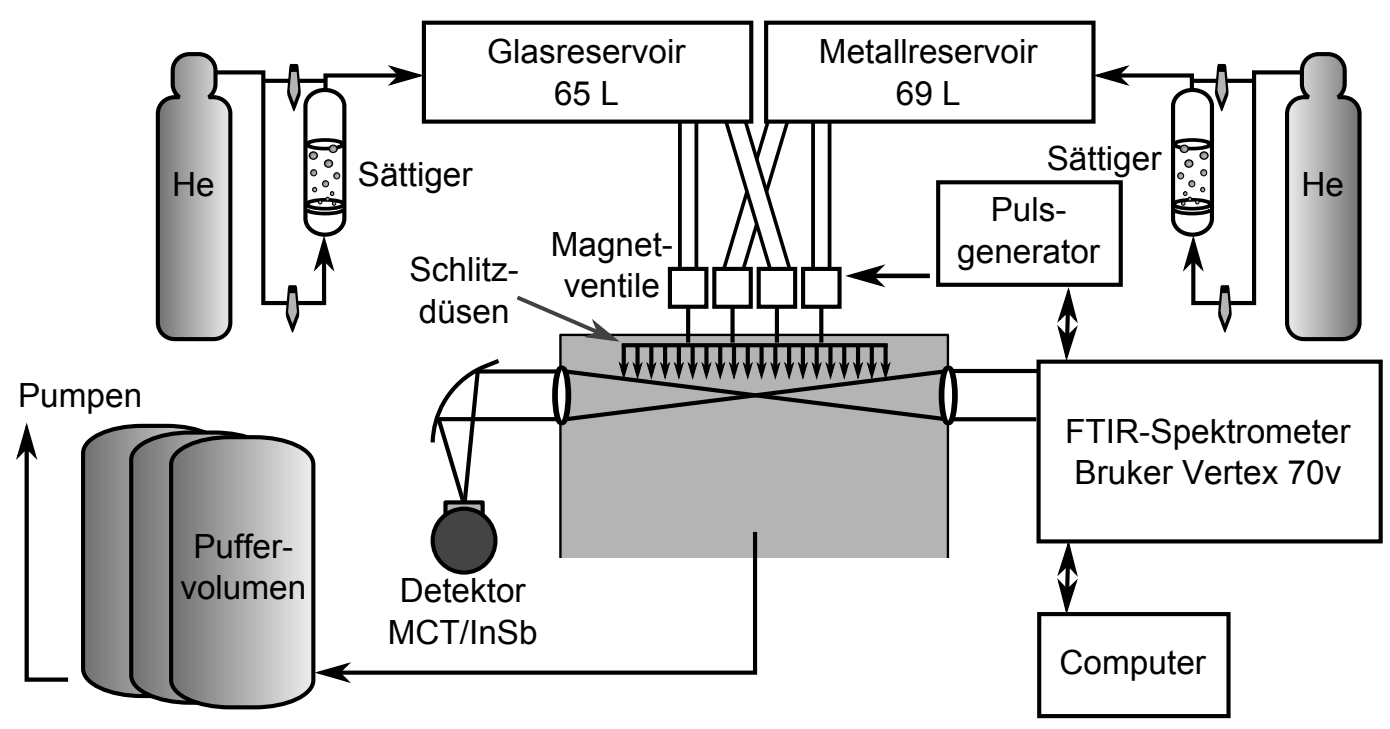

Abbildung 3.1: Schematischer Aufbau des Muesli-Jets.

\subsection{Muesli-Jet-Apparatur}

\subsubsection{Grundidee}

Der dem Muesli-Jet zugrunde liegende Gedanke ist die Verbindung eines relativ langen Absorptionswegs mit einfacher Justage des IR-Strahls und den Möglichkeiten der Generierung warmer Expansionen sowie der synchronen Expansion zweier nicht vorgemischter Komponenten mit einem variablen Abstand zwischen Düsenausgang und Infrarotstrahl zur Untersuchung der Dynamik in Überschallexpansionen. Dies wurde realisiert durch eine parallele Anordnung von zwanzig Schlitzdüsen, die jeweils senkrecht zum IR-Strahl angeordnet sind und abwechselnd mit verschiedenen Gasmischungen beschickt werden können. Dieser getrennte Einlass bei gleichzeitiger räumlicher Nähe der Expansionen soll die Analyse der Prozesse bei der Clusterbildung im Überschallstrahl ermöglichen und gleichzeitig die bei einer Vormischung vorgelagerten Prozesse wie Isotopenaustauschreaktionen unterbinden. Das Akronym Muesli (,multiple equidistant slits“) ergibt sich aus der äquidistanten Anordnung der zwanzig Schlitzdüsen in der ursprünglichen Düsenplatte und weist zudem auf die Mischung unterschiedlicher Substanzen hin. ${ }^{59,60}$

\subsubsection{Aufbau}

Abbildung 3.1 zeigt den schematischen Aufbau der Muesli-Jet-Apparatur. Da hier die getrennte Zuführung von zwei Komponenten zur Multischlitzdüse ermöglicht werden muss, existieren sowohl zwei Reservoire als auch zwei Sättiger. Die Reservoire weisen mit $65 \mathrm{~L}$ (Glasreservoir) und $69 \mathrm{~L}$ (Metallreservoir) ähnliche Volumina auf. Beide be- 


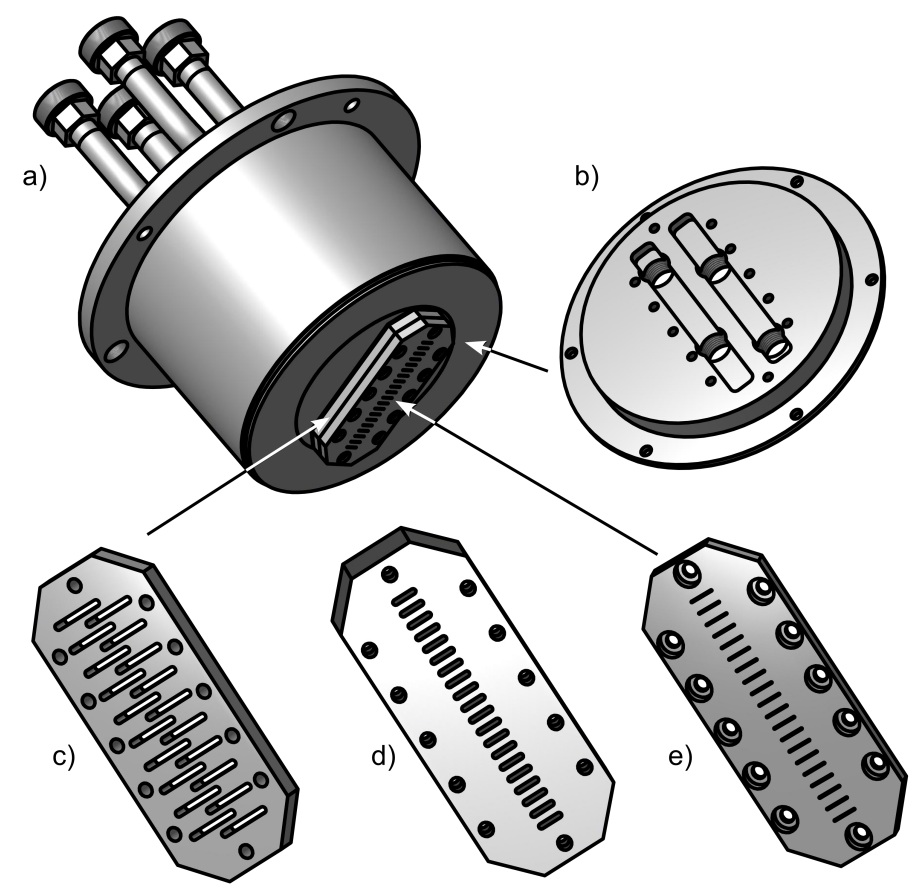

Abbildung 3.2: Düsenkonstruktion des Muesli-Jets unter Verwendung äquidistanter, paralleler Schlitzdüsen. a) Düsentopf mit vier Zuleitungen und zusammengebauter Düse, b) Zufuhrplatte, c) Kanalplatte, d) Düsenplatte (Innenseite), e) Düsenplatte (Außen- / Austrittsseite).

sitzen zur Befüllung drei Magnetventile, so dass die Gaszufuhr aus den Sättigern oder aus direkt angeschlossenen Edelgas- oder vorgemischten Gasflaschen flexibel und in beliebigen Kombinationen erfolgen kann. Die Weiterleitung der Gasgemische von den Reservoiren verläuft über insgesamt vier Edelstahl-Wellschläuche, von denen wahlweise alle vier an das Metallreservoir oder je zwei an Metall- und Glasreservoir angeschlossen werden können. Vier Magnetventile (Parker Lucifer, 221J3301E-299560-483816C2, $24 \mathrm{~V}=14 \mathrm{~W}$, Nennweite $8 \mathrm{~mm}$ ) kontrollieren den Gasfluss durch die Düsenplatte. Über eine Druckanstiegsuntersuchung in einem geschlossenen Volumen wurde sichergestellt, dass der Gasdurchlass durch alle vier Ventile bei gleichen Bedingungen etwa gleich ist. In der Gaszufuhr vom Reservoir zu den Düsen stellen die Magnetventile mit einem Durchlassquerschnitt von jeweils etwa $50 \mathrm{~mm}^{2}$ den Flaschenhals dar. Aufgrund der gesamten Schlitzdüsenfläche von $20 \times(10 \mathrm{~mm} \times 0.5 \mathrm{~mm})=100 \mathrm{~mm}^{2}$ ist eine Zufuhr durch nur zwei Magnetventile nicht ausreichend, so dass hierin die Verwendung von vier Zuläufen begründet liegt.

Der genaue Aufbau der Multischlitzdüse ist in Abbildung 3.2 dargestellt. Über die vier Zuleitungen gelangen die Gasgemische durch die Zufuhrplatte (Abb. 3.2 b) in zwei getrennte Volumina der Abmessungen $(105 \times 12 \times 5) \mathrm{mm}^{3}$ und verteilen sich dort. Die sich anschließende Kanalplatte (Abb. 3.2 c) stellt die Verbindung zwischen der 
Zufuhr- und der eigentlichen Düsenplatte dar. Sie stellt über ihre zwanzig Kanäle $\left((2.5 \times 19.5 \times 14) \mathrm{mm}^{3}\right)$ sicher, dass die getrennten Gasmischungen abwechselnd in die zwanzig Schlitzdüsen der Düsenplatte gelangen. Diese weist in ihrer Originalkonstruktion ${ }^{60}$ zwanzig parallele Schlitze in jeweils $5 \mathrm{~mm}$ Abstand zueinander auf. Die Dimension jedes Schlitzes beträgt auf der Niederdruck-/Auslassseite $10 \mathrm{~mm} \times 0.5 \mathrm{~mm}$ (Abb. $3.2 \mathrm{e}$ ), auf der der Kanalplatte zugewandten Seite sind sie jedoch breiter (Abb. 3.2 d). Zwischen den Düsenplatten befinden sich Viton-Flachdichtungen. Experimentelle Tests weisen darauf hin, dass diese bei größeren Druckdifferenzen ( $\geq 250$ mbar) eine Vermischung der zuvor getrennten Gase zulassen, daher wurde vorwiegend unter Verwendung gleicher Drücke in beiden Gaszufuhrsystemen gearbeitet. Der gesamte Düsenaufbau (Abb. 3.2 a) ist innerhalb der Apparatur stufenlos höhenverstellbar, so dass der Abstand zwischen Düsenausgang und Infrarotlicht variiert werden kann.

Das durch die Düse strömende Gas gelangt in insgesamt sechs Pufferbehälter mit einem Gesamtvolumen von $12 \mathrm{~m}^{3}$ (Abb. 3.1). Durch das große Volumen wird der Hintergrunddruck auch während intensiver Gaspulse gering gehalten ( $\ll 1 \mathrm{mbar}$ ). Sie werden durch ein Pumpensystem bestehend aus zwei Wälzkolbenpumpen (WKP 2000 AD, WKP 250 A) und einer Rotationsvakuumpumpe (UniDry 050-3) mit einer Gesamtpumpleistung von $2500 \mathrm{~m}^{3} \mathrm{~h}^{-1}$ evakuiert.

Als Infrarotspektrometer wird ein Bruker Vertex 70v mit RockSolid-Interferometer verwendet. Der austretende parallele Infrarotstrahl wird über einen goldbeschichteten Spiegel $(\varnothing 75 \mathrm{~mm})$ zur Düse umgelenkt. Zwei Calciumfluoridlinsen $(f=170 \mathrm{~mm})$ fokussieren und rekollimieren den Strahl unterhalb der Schlitzdüsenausgänge, senkrecht zur Orientierung der einzelnen Schlitze. Anschließend wird der wieder parallele Lichtstrahl in eine evakuierte Detektorkammer geleitet und mittels eines Parabolspiegels durch einen wechselbaren optischen Bandpassfilter auf einen $2 \mathrm{~mm} \varnothing$ Sandwich MCT/InSbDetektor fokussiert. Die Zwischenräume vor und hinter den Fokussierlinsen sind trockenluftgespült um Absorptionen durch atmosphärisches Wasser und Kohlenstoffdioxid zu minimieren.

Versuche haben ergeben, dass der vor dem Düsenausgang fokussierte IR-Strahl einen minimalen Durchmesser von mindestens $10 \mathrm{~mm}$ aufweist. Aufgrund der Länge der Düsenplatte von $126 \mathrm{~mm}$ ist an den äußeren Schlitzen von einem noch deutlich breiteren Strahl auszugehen (siehe Abb. 3.1), so dass generell eine recht große Zone der Expansion mit jeder Messung erfasst wird. Dies erschwert die Angabe eines Abstandes zwischen IR-Strahl und Düse. Unter der Annahme eines Abstandes der $\mathrm{CaF}_{2}$-Linsen von der doppelten Brennweite $2 \cdot f=34 \mathrm{~cm}$, einer Länge der Düsenplatte von $12.6 \mathrm{~cm}$, einem Durchmesser des parallelen Strahls aus dem Spektrometer von $4 \mathrm{~cm}$ und einem Strahldurchmesser im Fokus von $1 \mathrm{~cm}$ beträgt der Abstand des Mittelpunktes des Infrarotstrahls vom Düsenausgang wenn gerade noch keine Lichtabschwächung durch Reflexion an der Düsenplatte auftritt etwa $1 \mathrm{~cm}$. Dieser Punkt ist experimentell gut bestimmbar, daher erfolgt an dieser Stelle die Definition, dass sich die Angabe von Düsenabständen $h$ auf die für die Messungen verwendeten Abstände zwischen zwischen Düsenausgang und IR-Strahl-Mitte bezieht, mit dem eben berechneten Abstand als Referenzpunkt. 


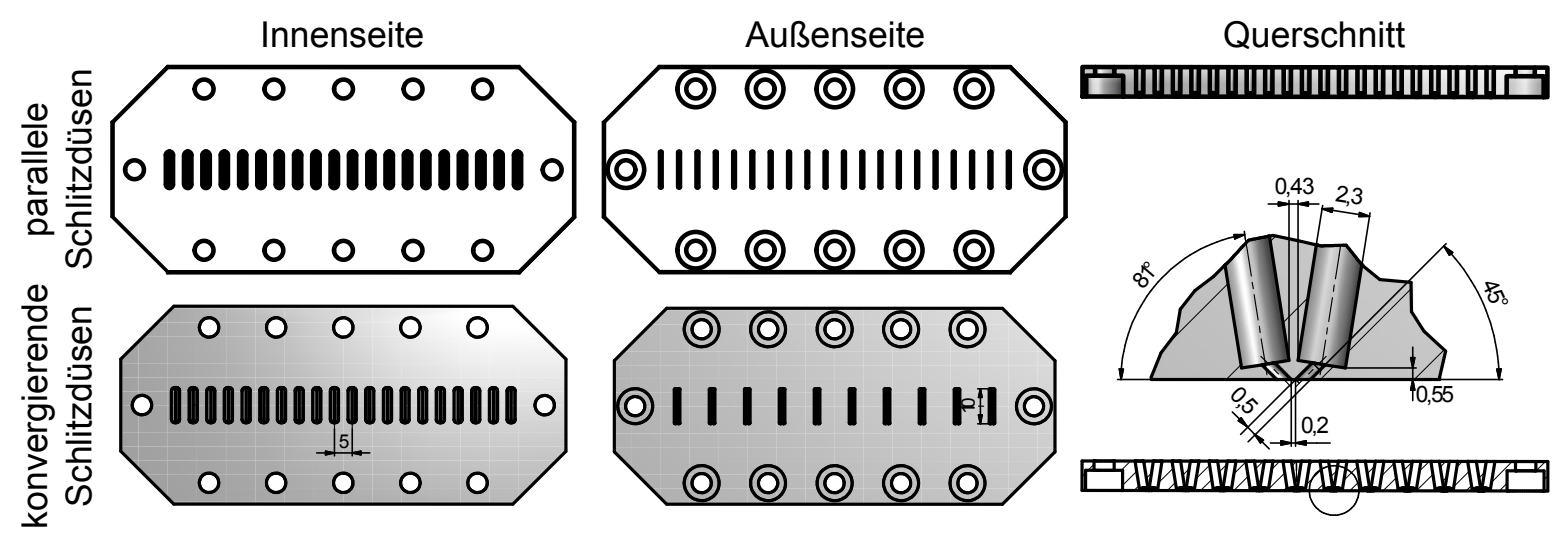

Abbildung 3.3: Düsenplatten der originalen (oben) und der für die Bildung von gemischten Aggregaten optimierten Mehrfachschlitzdüse (unten). Links: Der Kanalplatte zugewandte Innenseite der Düsenplatte (identisch für beide Versionen). Mitte: Außen/ Austrittsseiten der Düsenplatten mit paarweiser Anordnung für die neue Schlitzdüse. Rechts: Querschnitte der Düsenplatten (Austrittsseite unten). Für die konvergierende Schlitzanordnung ist eine Ausschnittsvergrößerung für ein Schlitzpaar gezeigt. Abstandswerte sind in mm angegeben.

Abstandsangaben unterhalb von $1 \mathrm{~cm}$ gehen daher mit einer teilweisen Blockierung des Infrarotstrahls und einer entsprechenden Abschwächung und Deformation einher und zeichnen sich aus diesem Grund durch ein geringeres Signal-zu-Rauschen-Verhältnis aus.

Die Steuerung der Spektrenaufnahme erfolgt über das Messmakro muesliMEAS.mtx, die Spektrenmittelung und Fouriertransformation mit dem muesliAV.mtx Auswertemakro. Die Makros sind im Anhang (Abschnitt A.7.1) zu finden. Die Synchronisation der Spektrometerscans mit den Gaspulsen wurde durch die trs-Methode 012-20-2-1-11.trs (Anhang A.6.1) in Verbindung mit dem Pulsgenerator Iota One (Parker Hannifin) gesteuert. Die jeweiligen Pulsverzögerungen und Öffnungszeiten der Magnetventile wurden manuell an die experimentellen Bedingungen (Auflösung, Scanmodus, Spiegelgeschwindigkeit) angepasst. Die resultierenden Zeiten für unterschiedliche Sätze an Messparametern sind in Abschnitt A.6.2 aufgelistet.

\subsubsection{Düsengeometrien}

Der modulare Aufbau der Muesli-Jet-Düse ermöglicht den schnellen Austausch der Düsenplatten und legt so Versuche mit verschiedenen Düsenanordnungen nahe. Während die Originaldüse mit zwanzig parallelen, äquidistanten Schlitzen sich bereits in der Vergangenheit als geeignet für die Erzeugung warmer Aggregate herausgestellt hat ${ }^{60}$ und diese Fähigkeit in dieser Arbeit weiter ausgereizt wurde (siehe Abschnitt 4.2), bewirkt sie jedoch bei der parallelen Expansion von Methanol und tert-Butylalkohol keine Bildung gemischter Dimere (Kapitel 4.1.1), wie sie bei vorgemischten Expan- 
sionen von Emmeluth et al. ${ }^{104}$ gefunden wurden. Als Ursache hierfür kann die Bildung einer Art Schichtstruktur der benachbarten Expansionen gesehen werden, so dass für jede einzelne Schlitzdüse die Zone freier Expansion durch eine warme Kollisionszone von der nächstliegenden Expansion getrennt ist. Einen Hinweis hierauf liefern die Fluoreszenz-Visualisierungen der Multilochdüsen-Expansionen von Herman et al. ${ }^{24}$. Demnach entsteht keine Durchdringung oder Überkreuzung der Expansionsfächer, sondern es findet vermutlich ein Ausweichen der Expansionen in Schlitzrichtung statt. Dieser Widerspruch zu den bei früheren Messungen ${ }^{60}$ gefundenen Mischclustern von 2,2,2-Trifluorethanol und Wasser ist vermutlich auf die dort verwendeten unterschiedlichen Drücke in den beiden Reservoiren von 400 und 1000 mbar zurückzuführen. Bei derartigen Druckdifferenzen ist eine Unterdrückung der Vermischung der Komponenten zwischen den Düsenplatten vermutlich nicht mehr möglich, so dass hier eine Art Vormischung stattfand.

Um dennoch in der Expansion gebildete gemischte Aggregate untersuchen zu können, muss folglich die Ausbildung von trennenden Gasschichten zwischen benachbarten Expansionen verhindert werden. Das aus diesem Ansatz resultierende Neudesign der Düsenplatte ist in Abbildung 3.3 zu sehen. Während die Innenseite der Düsenplatte weiterhin so aufgebaut ist, dass eine Gaszufuhr durch die gleiche Kanalplatte wie bei der alten Konstruktion erfolgen kann, laufen die Schlitzdüsen nun paarweise aufeinander zu, so dass die Abstände an der Austrittsseite alternierend etwa 10 und $0.2 \mathrm{~mm}$ (technisch bedingter Minimalwert) betragen. Die Schlitzdimensionen von $10 \times 0.5 \mathrm{~mm}^{2}$ werden hierbei beibehalten. Direkt vor dem Austritt in Richtung Expansionskammer weisen die Schlitzdüsen einen Winkel von $45^{\circ}$ relativ zur Düsenplatte auf. Auf diese Weise sollen die Expansionen der unterschiedlichen Komponenten nicht nur räumlich möglichst dicht zusammen gebracht werden, ohne jedoch eine Durchmischung vor dem Düsenausgang zuzulassen, sondern aufgrund der Impulsrichtungen auch zu einer Überlappung gezwungen werden. Wie in Abschnitt 4.1 dargestellt, konnte auf diese Weise tatsächlich eine Bildung von Mischclustern mit gleichzeitiger Unterdrückung von Isotopenaustauschreaktionen erzielt werden.

\subsubsection{Vorversuche zur Charakterisierung von Multischlitzdiusenexpansionen}

Die Eigenschaften von Multischlitzdüsenexpansionen sind im Hinblick auf Temperaturen und Clusterbildung noch nicht hinreichend untersucht. Daher erfolgt an dieser Stelle ein Vergleich zwischen den beiden verschiedenen Schlitzanordnungen des Muesli-Jets sowie eine Gegenüberstellung mit Spektren aus klassischen Einfachschlitzdüsenexpansionen und eine Untersuchung der Auswirkungen verschiedener Stagnationsdrücke. Auf eine tiefergehende Interpretation der Spektren soll hier jedoch verzichtet werden, da diese in Kapitel 4 ausführlich vorgenommen wird. Detaillierte Informationen zu den 


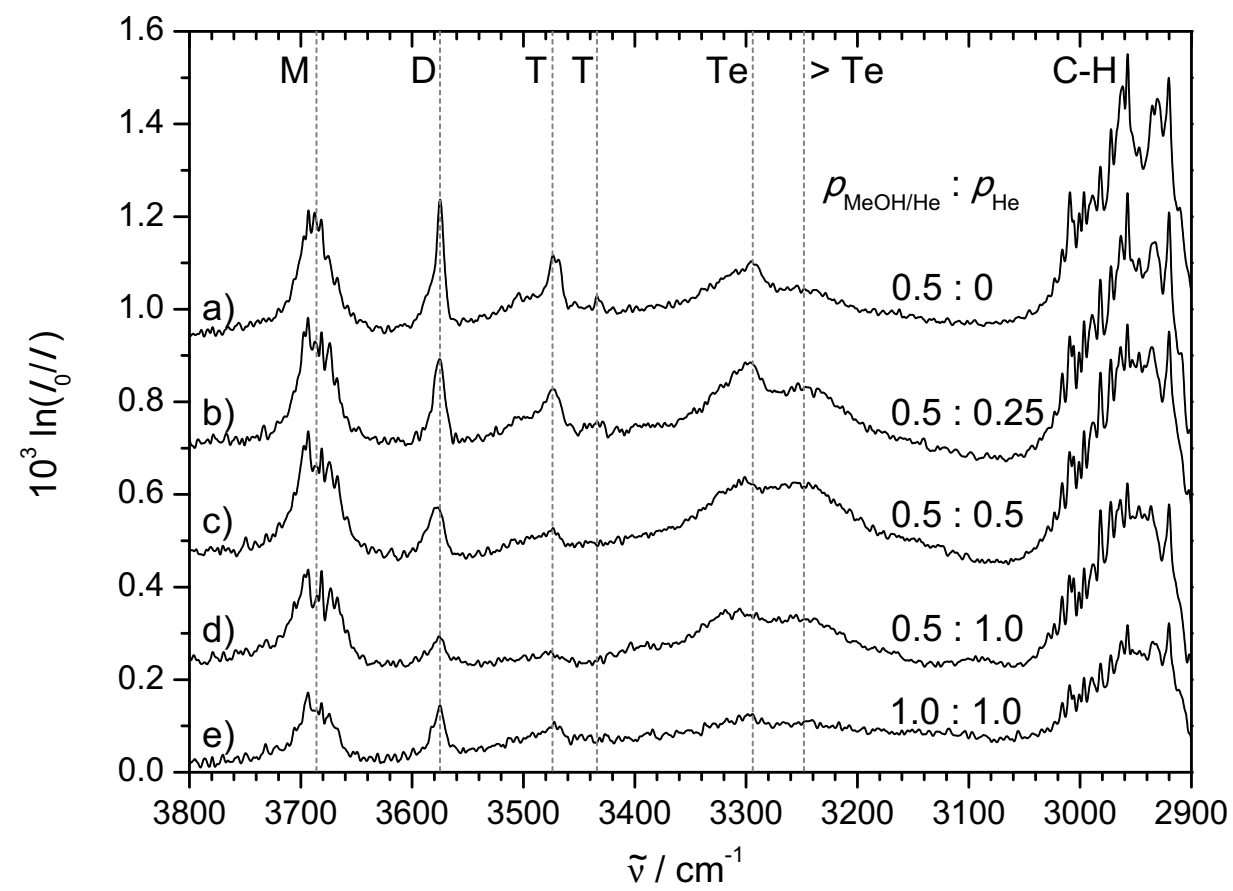

Abbildung 3.4: Einfluss von Stagnationsdrücken auf die O-H- und C-H-Streckschwingungsbanden bei Expansionen unter Verwendung der parallelen Schlitzdüsen. Expansion von Methanol in Helium durch eine Hälfte der Schlitzdüsen und von reinem Helium durch die anderen 10 Schlitze bei variablen Reservoirdrücken. Druckangaben in bar. Messabstand $h=1 \mathrm{~cm}, \mathrm{M}$ : Monomer, D: Dimer, T: Trimer, Te: Tetramer. Bandenpositionen wurden Referenz 105 entnommen.

verwendeten experimentellen Parametern können dem Spektrenverzeichnis und den Parameterblöcken im Anhang (A.3, A.5) entnommen werden.

\section{Stagnationsdrücke}

Abbildung 3.4 zeigt einen Vergleich der Expansionen von Methanol in Helium und reinem Helium durch jeweils 10 Schlitze der parallelen Muesli-Jet-Düsenplatte bei verschiedenen Verhältnissen der Stagnationsdrücke. Ausgehend von einer Methanol-Expansion durch 10 Schlitze (Abb. 3.4 a) wird eine zunehmende Menge an reinem Helium durch die dazwischenliegenden Schlitzdüsen expandiert (Abb. 3.4 b-d). Hierbei sollte die Menge an Methanol in der Expansion konstant bleiben, während eine zunehmende Störung durch die Helium-Expansionen erfolgt. Als Maß für die MethanolGesamtkonzentration im untersuchten Bereich der Expansion kann die Intensität der C-H-Streckschwingungsbanden $\left(\approx 3050-2900 \mathrm{~cm}^{-1}\right)$ verwendet werden, da diese nur geringen Einflüssen durch Clusterbildungen unterliegen. Bei derartigen Vergleichen muss jedoch beachtet werden, dass bei unterschiedlichen Stagnationsdrücken der bei- 
den Komponenten die zwischen den Düsenplatten liegenden Flachdichtungen einen Gasdurchlass unter Umständen nicht vollständig verhindern können.

Im Spektrum der Methanol-Expansion mit 0.5 bar durch jede zweite Schlitzdüse (Abb. 3.4 a) sind relativ starke, definierte Banden für Clustergrößen vom Monomer bis zum Tetramer zu erkennen. Die Bande bei $3248 \mathrm{~cm}^{-1}$ hat ihren Ursprung in Aggregaten, die mindestens fünf Methanol-Moleküle enthalten. ${ }^{3}$ Wenn zusätzlich eine Helium-Expansion mit dem halben (Abb. 3.4 b) oder gleichen (Abb. 3.4 c) Stagnationsdruck durch die zwischenliegenden Schlitze erfolgt, ist im Bereich der C-H-Streckschwingungen zunächst kein Einfluss zu erkennen. Die Menge an Alkohol-Molekülen im durch den IRStrahl geprobten Zylinder der Expansion bleibt also konstant, wie es aufgrund der unveränderten Methanol-Expansionen auch zu erwarten ist. Im O-H-Streckschwingungsbereich sind jedoch deutliche Veränderungen zu erkennen. Bei zunehmendem HeliumDruck nimmt die Halbwertsbreite der Monomer-Bande zu und zeigt so die Anregung einer höheren Zahl von Rotationsniveaus. Die nebenliegenden Helium-Expansionen vermindern also die Abkühlung in den methanolhaltigen Expansionen. Die Intensitäten der Dimer- und Trimer-Banden sinken bei zunehmenden Helium-Drücken deutlich ab. Auch dies kann als Effekt der Erwärmung verstanden werden, da die Dissoziationsenergie von Methanol-Dimeren mit $D_{0} \approx 13 \mathrm{~kJ} \mathrm{~mol}^{-1}$ relativ gering ist ${ }^{106}$ und die cyclischen Trimere ein hohes Maß an Ringspannung aufweisen und daher ebenfalls eine deutlich geringere Stabilität als beispielsweise Tetramere aufweisen. Gleichzeitig ist wie beim Monomer eine Verbreiterung der Banden zu erkennen. Im Gegensatz zu diesen kleinen Aggregaten ist für Tetramere und insbesondere noch größere Cluster jedoch eher eine Intensitätszunahme als ein Abfall zu erkennen. Aufgrund der stärkeren Kooperativität im Vergleich zum Trimer sind sie auch bei höheren Temperaturen stabil. Die vermehrte Bildung größerer Cluster weist zudem auf eine erhöhte Anzahl von Stößen zwischen Methanol-Molekülen hin und legt daher eine Kompression der Methanol-Expansionen durch die zwischenliegenden Helium-Expansionen nahe.

Wird der Helium-Druck weiter auf das Doppelte des Stagnationsdrucks der Methanol-Helium-Mischung erhöht (Abb. 3.4 d), tritt ein zusätzlicher Effekt auf. Zwar erfolgt noch eine weitere Erwärmung der Expansion, erkennbar an der Verbreitung der Monomer-Bande, und auch die Dimer- und Trimerbanden zeigen gegenüber den Banden größerer Cluster weiterhin eine ausgeprägtere Intensitätsabnahme. Jedoch ist gleichzeitig eine Abnahme der Gesamtintensität der C-H-Streckschwingungsbanden (und auch der $\mathrm{O}-\mathrm{H}-$-Streckschwingungsbande des Monomers) zu erkennen, gleichbedeutend mit einer Abnahme der Methanol-Menge im geprobten Bereich der Expansion. Als Ursache hierfür kann eine weiter verstärkte Verformung der Methanol-Expansion durch den höheren Druck der Helium-Expansionen in Betracht gezogen werden. Hierdurch würde eine Verbreiterung der MeOH-Expansionen in Schlitzrichtung und somit aus dem durch den IR-Strahl erfassten Bereich heraus erfolgen.

Spektrum e) in Abbildung 3.4 wurde bei Stagnationsdrücken beider Reservoire von 1.0 bar aufgenommen. Da beide Drücke gleich sind, sollte hier eigentlich keine übermäBige seitliche Verdrängung der Expansionen in Schlitzrichtung auftreten. Dennoch liegt 
die Intensität der C-H-Streckschwingungsbande noch unter der in Spektrum d), trotz einer Erhöhung des Drucks der Methanol-Helium-Mischung. Dieses kontraintuitive Verhalten ist vermutlich auf die Art der Reservoirbefüllung zurückzuführen. Nach einem Gaspuls strömt dabei solange Helium durch das temperierte Methanol im Sättiger, bis der eingestellte Soll-Druck im Reservoir wieder erreicht ist. Es ist davon auszugehen, dass der größte Methanol-Anteil zu Beginn des Nachfüllprozesses ins Reservoir gelangt, da sich während der Pausen zwischen den Pulsen der Methanol-Gleichgewichtsdampfdruck im Sättiger einstellen kann. Da beim Nachfüllen jedoch ein Vielfaches des Sättigervolumens nachströmt, ist der Übertritt von Methanol in die Gasphase nicht schnell genug um dauerhaft die Gleichgewichtskonzentration einzustellen, wodurch die Substanzkonzentration im Helium sinkt. Bei einem höheren Stagnationsdruck steigt auch die Menge des pro Puls expandierten Gases und somit die Dauer des Nachfüllprozesses. So entsteht bei höheren Stagnationsdrücken eine geringere Methanol-Konzentration im Reservoir. Die geringere Methanol-Menge in der Expansion führt zur relativen Bevorzugung der Bildung kleinerer Cluster und erklärt so die verhältnismäßig hohe Intensität der Dimerbande in Spektrum 3.4 e. Die geringere Expansionstemperatur, die sich aus dem höheren Stagnationsdruck und der damit verbundenen höheren Stoßanzahl mit Trägergasmolekülen ergibt, trägt zusätzlich zur Stabilisierung der Dimere bei.

Insgesamt führt also ein geringer Abstand nebeneinanderliegender Expansionen zu einer Erwärmung und einer Verformung der Expansionen. Hierdurch verringert sich die Bildung von Aggregaten mit schwachen intermolekularen Bindungen, während stabilere Cluster verstärkt entstehen. Bei einer Veränderung der Stagnationsdrücke besteht aufgrund des Ablaufs der Reservoir-Nachfüllung kein linearer Zusammenhang zwischen Druck und Substanzmenge. Es sind gleiche Werte für die Stagnationsdrücke in beiden Reservoiren anzustreben.

\section{Düsengeometrien}

Nach diesen Betrachtungen der Auswirkungen von Stagnationsdruckvariationen sollen nun die Expansionscharakteristika der verschiedenen Mehrfachschlitzdüsenanordnungen mit denen einer Einfachschlitzdüse verglichen werden. Der Aufbau der Apparatur mit einfacher Schlitzdüse (Ragout-Jet) ist in Referenz 10 beschrieben. Die Schlitzbreite von $0.5 \mathrm{~mm}$ entspricht der der Muesli-Jet-Düsen. Die Ausrichtung der Einfachdüse erfolgt parallel zum Infrarotstrahl, so dass mit einer Schlitzlänge von $120 \mathrm{~mm}$ auch der Gesamtabsorptionsweg vergleichbar zu dem der Multischlitzdüsen ist. Wird nur jede zweite der zwanzig Düsenöffnungen verwendet, so ist auch die Gesamtöffnung der betrachteten Düsen und somit der Gasdurchlass ähnlich.

Abbildung 3.5 zeigt die Spektren der Expansionen von Wasser in Helium bei etwa $0{ }^{\circ} \mathrm{C}$ Sättigertemperatur für die verschiedenen Düsengeometrien. Um eine Vergleichbarkeit des Gasdurchlasses bei gleichem Stagnationsdruck zu erhalten, wurde bei den Multischlitzdüsen nur jede zweite Düsenöffnung verwendet. Tatsächlich zeigt das Spektrum der Expansion durch die Einfachschlitzdüse (Abb. 3.5 a) im Vergleich zur paral- 


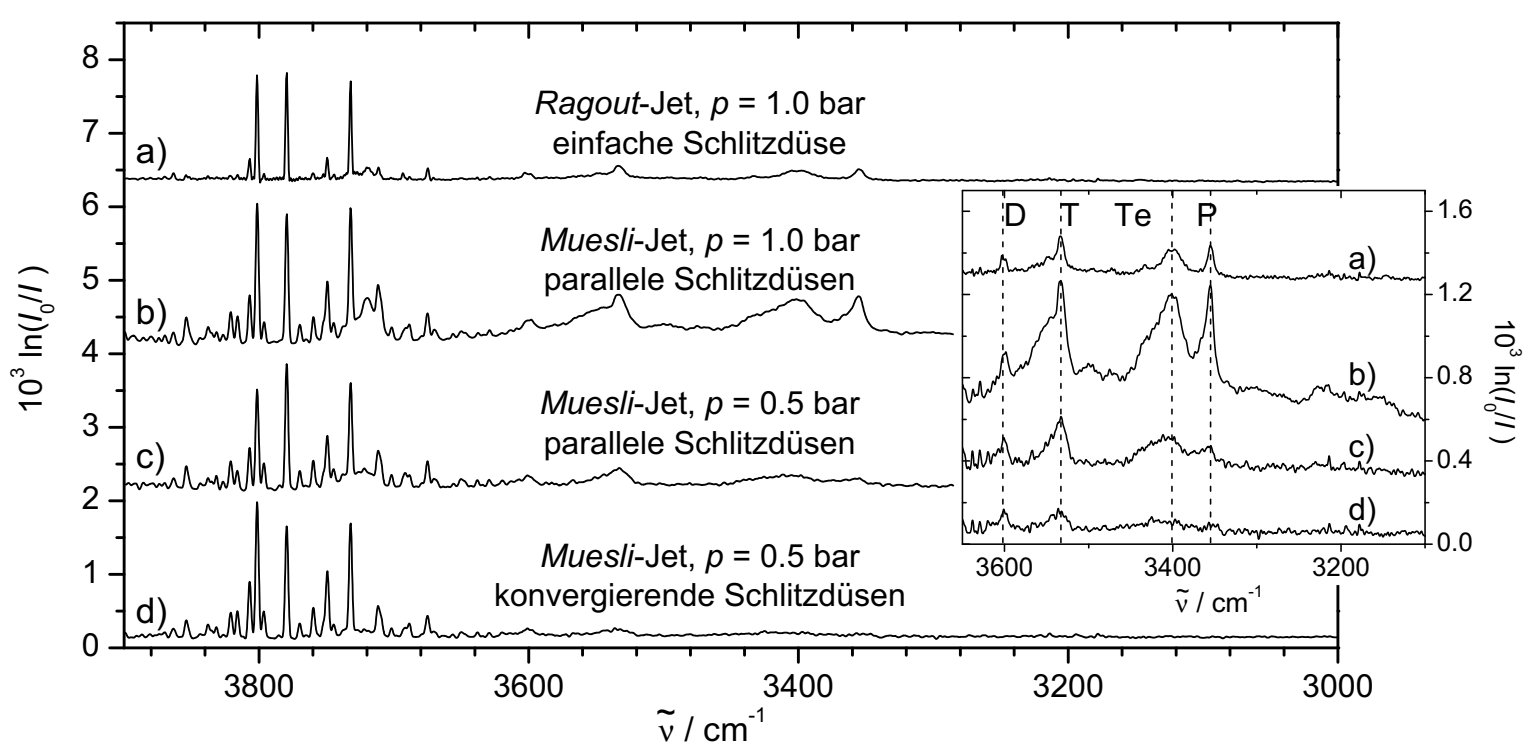

Abbildung 3.5: Unterschiede in der Clusterbildung bei verschiedenen Düsengeometrien und Stagnationsdrücken. Expansion von Wasser in Helium, Übersichtsspektrum im Bereich der $\mathrm{O}-\mathrm{H}$ Streckschwingungen und Ausschnittsvergrößerung der Clusterbanden. a) Expansion durch einfache Schlitzdüse $\left(120 \times 0.5 \mathrm{~mm}^{2}\right), \vartheta_{\mathrm{H}_{2} \mathrm{O}}=-2{ }^{\circ} \mathrm{C}, p_{\mathrm{He}}=1$ bar, Spektrum wurde Referenz 107 entnommen. b) Expansion durch jeden zweiten der parallelen Schlitze des Muesli-Jets, $\vartheta_{\mathrm{H}_{2} \mathrm{O}}=-1{ }^{\circ} \mathrm{C}, p_{\mathrm{He}}=1$ bar. c) Wie b), $\vartheta_{\mathrm{H}_{2} \mathrm{O}}=2{ }^{\circ} \mathrm{C}$, $p_{\mathrm{He}}=0.5$ bar. d) Expansion durch jeden zweiten der konvergierenden Schlitze des Muesli-Jets, $\vartheta_{\mathrm{H}_{2} \mathrm{O}}=2-5{ }^{\circ} \mathrm{C}, p_{\mathrm{He}}=0.5$ bar. Auflösung für alle Spektren: $2 \mathrm{~cm}^{-1}$. Düsenabstand $h=1 \mathrm{~cm}$ für Multidüsenexpansionen. Bandenpositionen wurden Referenz 5 entnommen.

lelen Anordnung von 10 Schlitzen (Abb. 3.5 b) auch nur eine geringfügig niedrigere Monomer-Konzentration. Die sehr viel höhere Anzahl von Monomer-Rotationslinien bei Verwendung der Mehrfachschlitzdüse weist auf eine deutlich höhere Rotationstemperatur hin, obwohl der Abstand zwischen den Schlitzdüsen mit $10 \mathrm{~mm}$ groß ist. Die Ausschnittsvergrößerung für den Bereich der O-H-Streckschwingungen der Wassercluster zeigt für Spektrum b) zum einen eine deutlich ausgeprägtere Aggregatbildung als im Einfachschlitzdüsenspektrum a). Zum anderen treten bei den Clusterbanden zusätzlich zu den scharfen Peaks breite, blauverschobene Schultern auf, die in Spektrum a) nicht erkennbar sind und aufgrund der geringen Bandenintensitäten um $3200 \mathrm{~cm}^{-1}$ auch nicht auf Hexamerbeiträge zurückführbar sind. Dies kann als Hinweis auf das Vorliegen von zwei unterschiedlichen Temperaturen und somit auch von zwei Zonentypen innerhalb der Expansion gesehen werden. Solch eine Koexistenz zweier unterschiedlicher Temperaturen wurde bereits für Multilochdüsenexpansionen beobachtet ${ }^{24}$, wo sie den Zonen freier Expansion (kalt) und den Kollisionszonen (warm) zugeordnet wurden.

Eine Senkung des Stagnationsdrucks für die parallelen Multischlitzdüsenexpansio- 
nen von 1.0 bar (Abb. 3.5 b) auf 0.5 bar (Abb. 3.5 c) bewirkt zwar eine Absenkung der Monomer- (und somit näherungsweise der Gesamt-)Konzentration, jedoch um deutlich weniger als einen Faktor zwei. Als Ursache hierfür kann erneut der Nachfüllprozess angesehen werden (siehe Abschnitt „Stagnationsdrücke“). Gleichzeitig sinken bei der Reduktion des Stagnationsdrucks die Clusterbandenintensitäten stärker als die Monomerintensitäten erwarten lassen. Hierbei ist der Intensitätsabfall für größere Aggregate stärker, da deren Bildung eine stärkere Abhängigkeit von der Gesamtkonzentration aufweist. Dies kann einerseits auf einen Mangel an Trägergasstoßpartnern zur Stabilisierung der Aggregate bei geringerem Stagnationsdruck zurückgeführt werden. Andererseits muss auch in Betracht gezogen werden, dass in Spektrum $3.5 \mathrm{~b}$ durch das hohe Maß an Clusterbildung ein prozentual signifikanter Verbrauch von Monomeren stattfindet, so dass deren Konzentration nicht mehr als proportional zur Gesamtkonzentration angesehen werden kann. Dies würde zu einer größeren Differenz der Wassermengen in den Spektren b) und c) führen und somit auch die Unterschiede in den Clusterbandenintensitäten unterstützen.

Einen Vergleich der Expansionen durch parallele und konvergierende Schlitzdüsenanordnungen bei gleichem Stagnationsdruck zeigen die Spektren 3.5 c und d. Da nur jede zweite der Düsen verwendet wurde, unterscheiden sich die Expansionen nur in dem Austrittswinkel der Schlitze relativ zur Ausbreitungsrichtung. Es zeigt sich, dass bei Expansionen durch die „schrägen“ Schlitzdüsen (Spektrum 3.5 d) im Vergleich zu den parallelen $(3.5 \mathrm{c}$ ) die Intensitäten der Clusterbanden geringer und eventuell die Temperaturen etwas höher sind. Dies könnte darauf zurückzuführen sein, dass der Unterschied zwischen Austritts- und Ausbreitungsrichtung eine Störung darstellt, die sowohl die stoßinduzierte Kühlung als auch die Aggregation verzögert, so dass bei den hier betrachteten geringen Düsenabständen deutliche Abweichungen zum Gasstrom durch die Schlitzdüsen in Ausbreitungsrichtung auftreten. Das Ausmaß dieser Unterschiede ist in Bezug auf die Clusterbildung jedoch eher unerwartet.

Insgesamt werden also die deutlich höheren Temperaturen in Mehrfachschlitz- gegenüber Einfachschlitzdüsenexpansionen ${ }^{59,60}$ bestätigt, eine Quantifizierung wird in Abschnitt 4.2 vorgenommen. Zudem wird nahegelegt, Untersuchungen hinsichtlich der Clusterbildung mit konvergierender Schlitzdüsenanordnung bei Abständen der Düsenöffnungen zum Zentrum des Infrarotstrahls von mehr als $1 \mathrm{~cm}$ durchzuführen.

Einen Vergleich der beiden verschiedenen Multischlitzdüsengeometrien im Standardbetrieb unter Verwendung aller zwanzig Düsen und zwei verschiedener Substanzen zeigt Abbildung 3.6. Als Testsubstanzen wurden Methanol (MeOH) und tert-Butylalkohol $(t \mathrm{BuOH})$ aufgrund ihrer bereits bekannten Spektren als Einzelsubstanzen und bei einer Vormischung ${ }^{104}$, dem Vorliegen jeweils nur eines Konformers und der barrierefreien Bildung gemischter Dimere gewählt. Die Alkohol-Konzentrationen im Trägergas Helium wurden für hohe Bandenintensitäten der $\mathrm{O}-\mathrm{H}-$ Streckschwingungen der Dimere optimiert und gleichzeitig so eingestellt, dass sich für die beiden zu vergleichenden Spektren 3.6 a und b nahezu gleiche Intensitäten der C-H-Streckschwingungsbanden $\left(3050-2900 \mathrm{~cm}^{-1}\right)$ ergaben. 


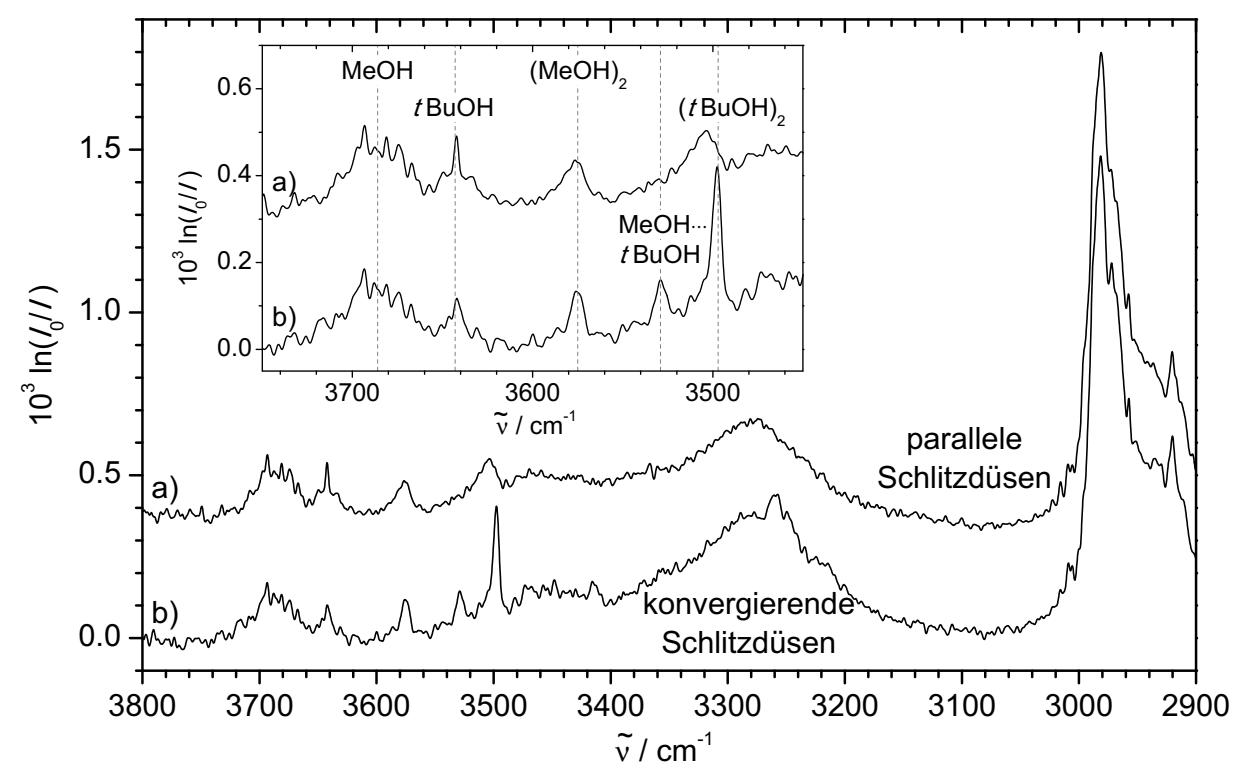

Abbildung 3.6: Vergleich von Mischexpansionen zweier Substanzen im Muesli-Jet unter Verwendung der beiden Düsengeometrien. Expansion von Methanol und tert-Butylalkohol durch jeweils jede zweite der zwanzig Schlitzdüsen. Trägergas Helium, Düsenabstand $h=1 \mathrm{~cm}$. Die Ausschnittsvergrößerung der Spektren zeigt den Bereich der O-HStreckschwingungen kleiner Alkoholcluster, Bandenpositionen wurden Referenz 104 entnommen. a) Parallele Schlitzdüsenanordnung. b) Konvergierende Schlitzdüsenanordnung.

Spektrum 3.6 a wurde mit der Muesli-Jet-Düsenplatte mit paralleler Schlitzanordnung aufgenommen, während für Spektrum 3.6 b das neue Düsendesign mit konvergierenden Schlitzdüsenpaaren verwendet wurde. Wie aufgrund der Übereinstimmung der C-H-Streckschwingungsintensitäten zu erwarten ist, stimmen auch die O-H-Intensitäten der Monomere bei $3686 \mathrm{~cm}^{-1}(\mathrm{MeOH})$ und $3643 \mathrm{~cm}^{-1}(t \mathrm{BuOH})$ in etwa überein. Für die Dimere ergeben sich hingegen deutliche Unterschiede, wie der Ausschnittsvergrößerung der Spektren in Abbildung 3.6 zu entnehmen ist. Beide Spektren zeigen Banden der Homodimere von Methanol und tert-Butylalkohol. Bei Verwendung der parallelen Schlitzdüsen tritt jedoch neben einer deutlich größeren Halbwertsbreite beider Banden auch eine Blauverschiebung der Dimerbande von tert-Butylalkohol um $8 \mathrm{~cm}^{-1}$ gegenüber dem mit einer Einfachschlitzdüse erhaltenen Wert ${ }^{104}$ von $3497 \mathrm{~cm}^{-1}$ auf, die für das Methanoldimer $\left(3575 \mathrm{~cm}^{-1}\right)^{104}$ nicht beobachtet wird. Die insgesamt wärmeren Expansionen bei paralleler Schlitzanordnung gegenüber den konvergierenden Schlitzen sind hierbei durch den geringeren Abstand der parallelen Düsenschlitze zueinander von $0.5 \mathrm{~cm}$ im Vergleich zu den konvergierenden Düsenpaaren $(\approx 1 \mathrm{~cm})$ zu erklären. Die Blauverschiebung des $t \mathrm{BuOH}$-Dimers weist auf eine Schwächung der Wasserstoffbrückenbindung durch die Erwärmung hin. Während ein derartiges, schwächer gebundenes Dimer durch die Dispersionswechselwirkung zwischen den tertiären Butylgruppen sta- 
bilisiert werden kann, führt eine analoge Schwächung der Wasserstoffbrückenbindung im Methanol-Dimer vermutlich zur Dissoziation, so dass hier keine Rotverschiebung der Bande im wärmeren Spektrum 3.6 erkennbar ist.

Zusätzlich ist in Spektrum 3.6 b eine Bande bei $3529 \mathrm{~cm}^{-1}$ zu erkennen, die bei paralleler Düsenanordnung nicht auftritt. Diese wurde bereits früher dem gemischten Dimer mit Methanol als Wasserstoffbrückendonor und tert-Butylalkohol als Akzeptor zugeordnet. ${ }^{104}$ Während bei parallelen Expansionen also selbst eine theoretisch barrierefreie Bildung von Mischclustern ausbleibt, sind bei den konvergierenden Schlitzdüsenpaaren gemischte Aggregate zu beobachten. Diese Düsengeometrie ist somit voraussichtlich auch geeignet für die Koexpansion zweier Substanzen, die bei einer Vormischung unerwünschte Reaktivität zeigen.

Im Bereich der größeren Alkohol-Cluster unterhalb von $3480 \mathrm{~cm}^{-1}$ zeigt sich deren verstärkte Bildung bei Verwendung der konvergierenden Schlitzdüsen. Diese kann sowohl auf die zusätzliche Intensität durch gemischte Aggregate als auch auf eine höhere Anzahl von Stößen zurückgeführt werden, die aus der erzwungenen Durchdringung von jeweils zwei Expansionen folgt. Genauere Charakteristika der Bildung von (Misch-) Clustern in variablen Abständen zur Düse und bei der Koexpansion verschiedener Isotopomere werden in den Abschnitten 4.1 und 4.3 untersucht und erläutert.

Insgesamt zeichnet sich die konvergierende gegenüber der parallelen Schlitzdüsenanordnung also durch eine Ermöglichung der Bildung von Mischclustern bei gleichzeitig kälteren Expansionen aus. Diese sind jedoch bei beiden Anordnungen deutlich wärmer als bei Einfachschlitzdüsen gleicher Schlitzbreite und Absorptionsstrecke.

\section{Konvergierende Schlitzdïsen}

Der Vergleich der Expansionen durch die verschiedenen Multischlitzdüsenanordnungen bei Verwendung nur jeder zweiten Düsenöffnung im Abschnitt „Düsengeometrien“ gab einen Hinweis auf einen eventuellen Vorteil größerer Düsenabstände bei Messungen mit der konvergierenden Düsenanordnung. Dies soll hier für den typischen Betrieb unter Nutzung aller zwanzig Schlitze überprüft werden.

Hierzu wurden Expansionen von Deuteriumoxid $\left(\mathrm{D}_{2} \mathrm{O}\right)$ in Helium verglichen, wobei das Gasgemisch auch bei Verwendung aller Düsenausgänge (und somit aller vier Zugänge) aus nur einem Reservoir entstammte. Spektren a) und b) in Abbildung 3.7 vergleichen noch einmal die Eigenschaften von Expansionen durch 10 oder 20 Schlitzdüsen. Hierbei ist zu beobachten, dass trotz der Verdoppelung der Gesamt-Düsenöffnungsfläche die Menge des $\mathrm{D}_{2} \mathrm{O}$-Monomers in etwa gleich bleibt. Dies ist - analog zur Erhöhung des Expansionsdrucks im Abschnitt „Stagnationsdrücke“ - durch den höheren Druckabfall im Reservoir während der Expansion durch zwanzig Schlitzdüsen und den bereits diskutierten Nachfüllprozess sowie durch Verdrängungseffekte zu erklären. Trotz der insgesamt ähnlichen $\mathrm{D}_{2} \mathrm{O}$-Menge in beiden Expansionen kann in Spektrum 3.7 a (mit 10 Schlitzen) gegenüber b (20 Schlitze) eine ausgeprägtere Clusterbildung beobachtet werden. Dies ist darauf zurückzuführen, dass aufgrund der paarweisen An- 


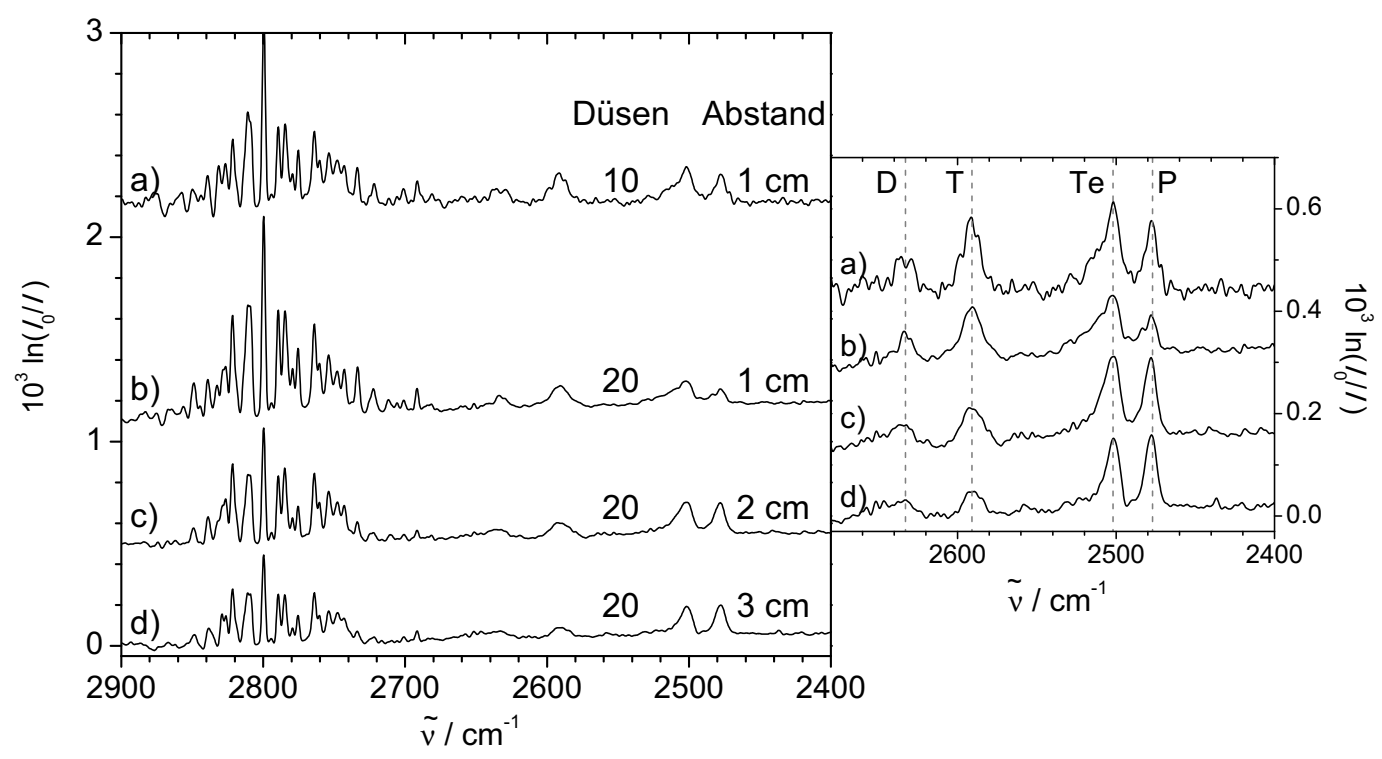

Abbildung 3.7: Spektren der Expansion von $\mathrm{D}_{2} \mathrm{O}$ in Helium durch die Muesli-Düsenplatte mit paarweise konvergierenden Schlitzen bei verschiedenen Abständen zur Düse. $p_{\mathrm{He}}=1000$ mbar. a) Expansion durch jede zweite der zwanzig Schlitzdüsen. $\mathbf{b}-\mathbf{d}$ ) Expansion durch alle Düsen aus einem Reservoir. Die Ausschnittsvergrößerung rechts zeigt den Bereich der O-H-Streckschwingungen der $\mathrm{D}_{2} \mathrm{O}-$ Cluster. D: Dimer, T: Trimer, Te: Tetramer, P: Pentamer; Bandenpositionen wurden Referenz 5 entnommen.

ordnung der Schlitzdüsen in Spektrum b) quasi eine Expansion mit doppelter Schlitzbreite im Vergleich zu a) stattfindet. Somit verteilt sich eine etwa gleiche Anzahl von $\mathrm{D}_{2} \mathrm{O}$-Molekülen auf die doppelte Fläche. Die folglich niedrigere $\mathrm{D}_{2} \mathrm{O}$-Dichte führt zu weniger Stößen zwischen den $\mathrm{D}_{2} \mathrm{O}-$ Molekülen, woraus ein geringerer Aggregationsgrad bei gleichem Düsenabstand von $1 \mathrm{~cm}$ folgt.

Dass die erzwungenen Überkreuzungen der benachbarten Expansionen dennoch eine verstärkte Aggregation zur Folge haben, kann bei höheren Messabständen zur Düse beobachtet werden. Trotz des Dichteabfalls der Expansion bei einer zunehmenden Entfernung zur Düse sind in Spektrum 3.7 c (Düsenabstand $2 \mathrm{~cm}$ ) ähnliche Intensitäten der Tetramer- und Pentamerbanden wie in Spektrum a) zu erkennen. Die Abstandsreihe der Spektren 3.7 b-d zeigt zudem, wie sich im Laufe der Expansion zusätzlich zum Dichteabfall zunehmend größere Aggregate bilden. Gleichzeitig weisen die abnehmenden Halbwertsbreiten der Clusterbanden auf eine Abkühlung hin.

Für die Beobachtung der Streckschwingungen wasserstoffbrückengebundener O-HGruppen erweist sich ein Abstand von $2 \mathrm{~cm}$ zur Düse als optimaler Kompromiss zwischen ausgeprägter Aggregation und hohen Bandenintensitäten und wird somit zum Standard für folgende Messungen. 


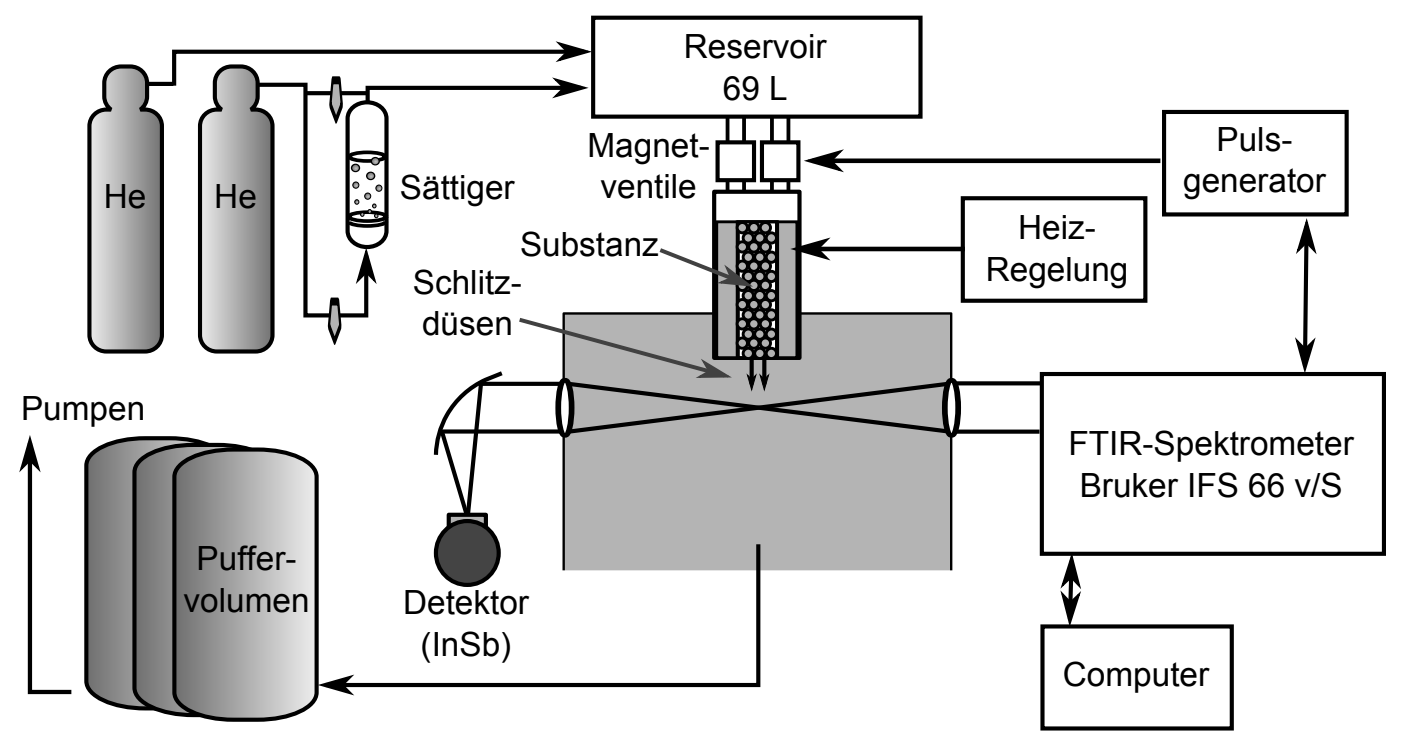

Abbildung 3.8: Schematischer Aufbau des Popcorn-Jets.

\subsection{Popcorn-Jet-Apparatur}

\subsubsection{Grundidee}

Zahlreiche für die Untersuchung intermolekularer Wechselwirkungen interessante Systeme weisen bei Zimmertemperatur einen geringen Dampfdruck auf. Um sie dennoch mit für die Jet-FTIR-Spektroskopie ausreichender Konzentration in ein Trägergas überführen zu können, ist eine Erwärmung dieser Substanzen über die Umgebungstemperatur nötig. Aufgrund der bereits in Abschnitt 3.1.1 angesprochenen Kondensation in kälteren Komponenten des Aufbaus (Reservoir, Zuleitungen) ist dies mit den üblichen Sättigersystemen nicht möglich. Der Aufbau des Popcorn-Jets umgeht diese Problematik durch eine separate, geheizte Probenkammer direkt vor dem Düsenausgang. Das Akronym Popcorn („poppet controlled resistively heated $\boldsymbol{n}$ ozzle“) ergibt sich aus dem Einsatz von Widerstandsheizungen und den die Probenkammer umschließenden Überström- (poppet-) Ventilen.

\subsubsection{Aufbau}

Der Aufbau der Popcorn-Jet-Apparatur erfolgte durch Dr. Corey A. Rice, Dr. Merwe Albrecht und Dr. Juhyon Lee und ist somit kein Bestandteil dieser Arbeit. Daher wird an dieser Stelle nur ein grober Überblick über die Komponenten und ihre Anordnung innerhalb der Apparatur gegeben, Details können der Literatur ${ }^{108,109,110}$ entnommen werden.

Der schematische Aufbau des Popcorn-Jets ist in Abbildung 3.8 zu sehen. Ein Edelstahlreservoir mit einem Volumen von $69 \mathrm{~L}$ kann über bis zu zwei Zugänge parallel 
mit Gasen oder Gasgemischen direkt aus Druckgasflaschen oder über den Umweg über einen Sättiger mit einer leichtflüchtigen Substanz befüllt werden. Der Gasdurchlass vom Reservoir zum Düsenaufbau erfolgt über zwei Magnetventile. Zwischen diesen Magnetventilen und dem Düsenausgang befindet sich die heizbare Probenkammer. Die zu untersuchende, schwerflüchtige Substanz wird auf Molekularsieb (Porendurchmesser $3 \AA$ ) gegeben oder aufgeschmolzen, in ein Glasrohr ( $\varnothing 20 \mathrm{~mm}$, Länge $70 \mathrm{~mm}$ ) gefüllt und die Öffnungen mit Glaswolle verschlossen. Das Probenröhrchen wird in der EdelstahlProbenkammer zwischen den zwei Überströmventilen platziert. Die Öffnungsdrücke der Überströmventile betragen 70 mbar an der Reservoirseite und 690 mbar an der Düsenseite, so dass sich das Trägergas während der gepulsten Expansionen zunächst in der Probenkammer aufstaut und einen Teil der schwerflüchtigen Substanz aufnimmt, bevor das Gemisch expandiert wird. Die Probenkammer ist von drei mit Heizdraht umwickelten Heizschalen umgeben, wobei die mittlere das Probenröhrchen temperiert und die beiden äußeren zur Vorheizung des Trägergases und zur Verhinderung der Substanzkondensation am Düsenausgang dienen. Das Heizsystem kann bis zu einer Probentemperatur von $180^{\circ} \mathrm{C}$ betrieben werden, wobei zur Vorheizung eine um $10^{\circ} \mathrm{C}$ und zur Kondensationsverhinderung eine um $20^{\circ} \mathrm{C}$ höhere Temperatur als die für die Probenheizung eingesetzte eingestellt wird. Das Gasgemisch wird durch eine Doppelschlitzdüse der Dimension $2 \times(0.5 \times 10) \mathrm{mm}^{2}$ mit einem Abstand der Schlitze zueinander von $10 \mathrm{~mm}$ expandiert. Dabei sind die Schlitze wie im Muesli-Jet orthogonal zum Infrarotstrahl ausgerichtet. Das Gasgemisch gelangt in ein Puffervolumen von $3.6 \mathrm{~m}^{3}$, welches durch ein Pumpensystem bestehend aus einer Sperrschieberpumpe $\left(250 \mathrm{~m}^{3} \mathrm{~h}^{-1}\right)$ und zwei Wälzkolbenpumpen (250 und $500 \mathrm{~m}^{3} \mathrm{~h}^{-1}$ ) evakuiert wird.

Als FTIR-Spektrometer wird ein Bruker IFS $66 \mathrm{v} / \mathrm{S}$ eingesetzt, dessen modulierter Infrarotstrahl mittels zwei beheizter Kaliumbromidlinsen fokussiert und wieder kollimiert wird. Dabei weist der Strahl am Brennpunkt einen Durchmesser von wenigen Millimetern auf und befindet sich etwa $5 \mathrm{~mm}$ vom Düsenausgang entfernt. Nach dem Austritt aus der Expansionskammer wird der Strahl über einen Parabolspiegel durch einen optischen Breitbandfilter auf einen $2 \mathrm{~mm} \varnothing \mathrm{InSb}$-Detektor innerhalb einer evakuierbaren Kammer fokussiert.

Der bei den Messungen erhaltene Multispektrenblock setzt sich aus 20 Hintergrundscans, 2 Vorscans, 1 Probenscan und $2 \times 1$ Nachscan zusammen. Zur Spektrenaufnahme und -auswertung werden die Makros Jet_Popcorn.mtx und JET_CALC.mtx verwen$\operatorname{det}^{110}$, ihr Aufbau entspricht im Wesentlichen dem der analogen Muesli-Jet-Makros.

Mit der Popcorn-Jet-Apparatur erzeugte Expansionen sind im Vergleich zu denen aus ungeheizten Einfachschlitzdüsen ${ }^{10}$ deutlich wärmer. Dies wird einerseits durch die höhere Gastemperatur vor der Düsenöffnung bedingt, andererseits aber auch (in geringerem Maße) durch die Interaktion der zwei Expansionen der Doppelschlitzdüse. Die Kombination aus Sättiger und beheizter Probenkammer ermöglicht neben der Untersuchung schwerflüchtiger Substanzen die gemischte Expansion von zwei Komponenten mit stark unterschiedlichen Dampfdrücken. 


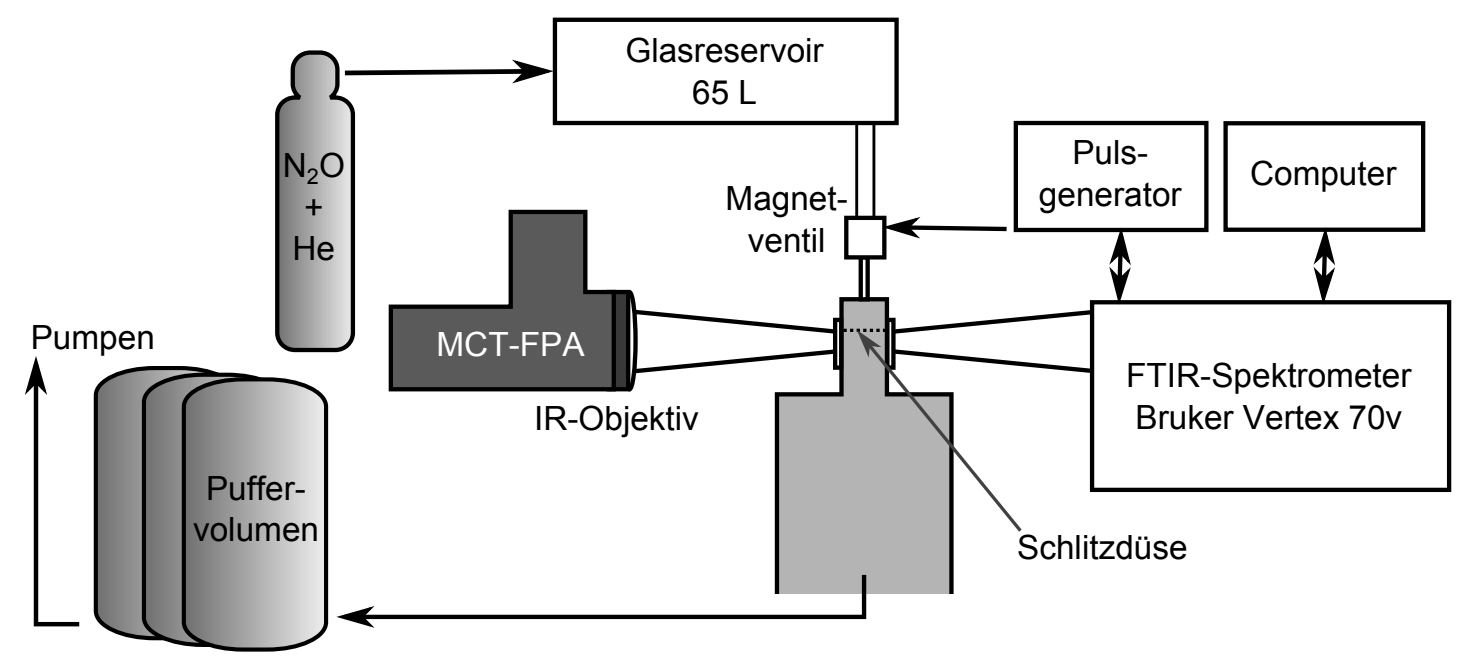

Abbildung 3.9: Schematischer Aufbau der Chilli-Jet-Apparatur.

\subsection{Chilli-Jet-Apparatur}

\subsubsection{Grundidee}

Die Struktur von Überschallexpansionen ist, wie in Abschnitt 2.3 gezeigt wurde, komplex. Dies führt in Verbindung mit den relativ großen Strahldurchmessern bei den eingesetzten FTIR-Techniken dazu, dass die erhaltenen Spektren immer eine Mittelung über verschiedene Expansionsbereiche darstellen und somit die Phase der Aggregatbildung am Anfang der Expansion, die Ruhezone, die lateralen Stoßfronten und warmes Hintergrundgas gleichzeitig in eine Messung eingehen können. Um nicht nur einen allgemeinen Überblick über die in der Überschallexpansion vorliegenden Spezies zu erhalten sondern genauere Aussagen über die Aggregationsdynamik, Dichte- und Temperaturentwicklungen treffen zu können, sind ortsaufgelöste Messungen erforderlich. Diese können eventuell auch Abschätzungen ermöglichen, ob und wann Messungen im Bereich der Reexpansionen hinter der ersten normalen Stoßfront lohnend sind. Um die zahlreichen Nachteile, die sich aus dem Einsatz von Blenden und Rastertechniken ergeben ${ }^{10}$ zu umgehen (siehe auch Kapitel 2.5), wurde hier der Ansatz für eine chemische Bildgebung durch Einsatz eines Focal Plane Array (FPA) Detektors in Kombination mit FTIR-Spektroskopie von Schlitzdüsen-Überschallexpansionen verfolgt. Hieraus ergibt sich auch das Akronym Chilli (chemical $\boldsymbol{i}$ maging by spatially resolved $\boldsymbol{i}$ nfrared spectroscopy).

\subsubsection{Aufbau}

Abbildung 3.9 zeigt schematisch den Aufbau des Chilli-Jets. Sowohl die Reservoire mit Befüllungsvorrichtungen als auch die Puffervolumina mit Pumpensystem, das Spektro- 
meter und die Pulsgenerierung sind identisch mit denen des Muesli-Jets. Details zu diesen Komponenten können daher Abschnitt 3.2.2 entnommen werden. Erfolgreiche Messreihen wurden bislang nur unter Verwendung von Lachgas in Helium als Testsubstanz durchgeführt, die Reservoirbefüllung erfolgte daher direkt aus Druckgasflaschen, die im Vorfeld im gewünschten Verhältnis befüllt worden waren. Die Gaszufuhr vom Reservoir zur Düse regelt ein Magnetventil (baugleich zu denen des Muesli-Jets). Weitere Experimente mit Chlorwasserstoff und Deuteriumoxid in Helium wiesen aufgrund des hohen Rauschlevels in den Spektren entweder nur direkt hinter der Schlitzdüse ausreichende Bandenintensitäten für folgende Auswertungen auf $(\mathrm{HCl})$ oder konnten überhaupt nicht nachgewiesen werden $\left(\mathrm{D}_{2} \mathrm{O}\right)$.

Im Spektrometer passiert der Infrarotstrahl einen für den betrachteten Spektralbereich geeigneten Bandpassfilter und gelangt dann ins Interferometer. Der modulierte, parallele Strahl mit einem Durchmesser von etwa $4 \mathrm{~cm}$ wird direkt vor Austritt aus dem Spektrometer mittels eines Parabolspiegels schwach auf die Düsenposition fokussiert, besitzt aber selbst im Fokus noch einen Durchmesser von etwa einem Zentimeter. Im Gegensatz zu den Messungen mit Einzelelementdetektor ist der hohe Strahldurchmesser für die Bildgebungsexperimente erwünscht, um eine ausreichend große Fläche der Expansion auszuleuchten. Nach dem Passieren der Düse wird der divergente Strahl mittels eines Objektivs auf einen $64 \times 64$ Pixel Quecksilber-Cadmium-Tellurid (MCT) Focal Plane Array Detektor (Santa Barbara Focalplane, SBF 161 MCT 64x64) fokussiert. Das Objektiv ist nur bis zu Wellenzahlen von etwa $3000 \mathrm{~cm}^{-1}$ ausreichend durchlässig, wodurch eine Untersuchung der O-H-Streckschwingungsbanden von Alkoholen oder Wasser ausgeschlossen wird. Die Kantenlänge der insgesamt 4096 Detektorelemente beträgt jeweils etwa $40 \mu \mathrm{m}$, so dass sich eine Gesamtdetektorfläche von etwa $2.5 \times 2.5 \mathrm{~mm}^{2}$ ergibt, zuzüglich geringfügiger Zwischenräume zwischen den einzelnen Pixeln.

Da - aufgrund der zur vollen Ausleuchtung der detektierbaren Fläche nötigen großen Apertur - auch bei Verwendung spektraler Filter eine Übersättigung der meisten Pixel eintritt, ist zusätzlich ein Lichtabschwächer notwendig. Eine flexible Lösung dieses Problems wurde durch den Einbau eines Rahmens in das Spektrometer realisiert, der mit einer beliebigen Anzahl von Lagen schwarzer Polyesternetze (typischerweise 2 bis 6) bespannt werden kann. Da der FPA-Detektor im Gegensatz zu den Einzelelementdetektoren nicht innerhalb einer evakuierbaren Kammer positioniert ist, sind die Zwischenräume zwischen Spektrometer und Düse bzw. Düse und Detektor mit einer trockenluftgespülten Röhrenkonstruktion umgeben, die Störbanden durch atmosphärisches Wasser und Kohlenstoffdioxid minimiert.

\subsubsection{Düsenkonstruktion}

Die Positionierung des Düsenkörpers des Chilli-Jets zwischen Spektrometer und FPADetektor kann Abbildung 3.10 a entnommen werden. Die Schlitzdüse hat eine Länge von $10 \mathrm{~mm}$ und eine Breite von $0.5 \mathrm{~mm}$ und ist parallel zum Infrarotstrahl ausgerichtet. 


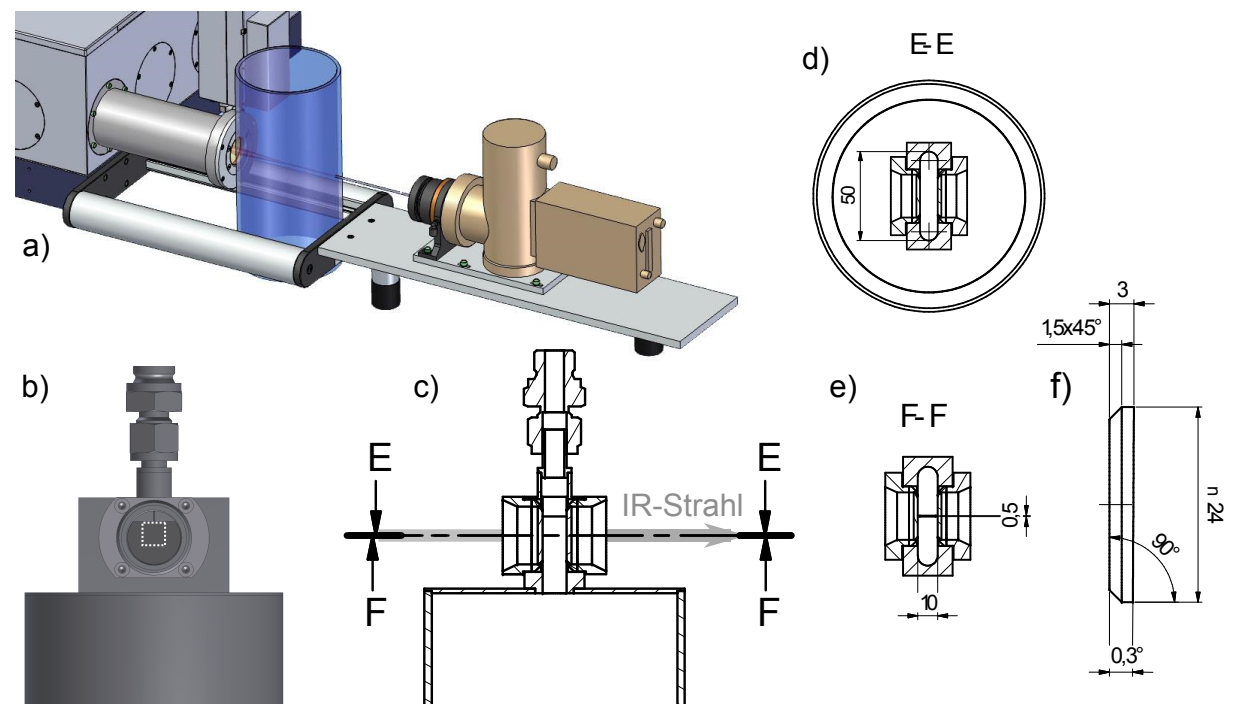

Abbildung 3.10: Aufbau der Düse des Chilli-Jets. a) Positionierung des Düsenkörpers (blauer Zylinder) zwischen Spektrometer und FPA-Detektor (Graphik erstellt von der Bruker Corporation). b) Frontale Ansicht der Schlitzdüsenkonstruktion, der weiße Rahmen kennzeichnet den geprobten Bereich von $1 \mathrm{~cm}^{2}$. c) Seitenansicht mit eingezeichnetem Strahlgang. d) Querschnitt an in c) eingezeichneter Linie, Ansicht von oben. e) Wie d), Ansicht von unten. f) Seitenansicht der Fenstergeometrie. Abstandsangaben in $\mathrm{mm}$.

Der seitliche Abschluss der Schlitzdüse erfolgt bündig durch zwei Bariumfluoridfenster (Abb. $3.10 \mathrm{c}$ und f). Die Wahl des Fenstermaterials ist durch den breiten Durchlässigkeitsbereich ${ }^{38}$ bis etwa $800 \mathrm{~cm}^{-1}$ und die geringe Wasserlöslichkeit von $\mathrm{BaF}_{2}$ bedingt.

Während der Expansionen steht dem Gasstrom ein Volumen mit rechteckigem Querschnitt von $10 \mathrm{~mm}$ in Düsenschlitzrichtung und $50 \mathrm{~mm}$ orthogonal dazu zur Verfügung. Dies ermöglicht eine freie seitliche Ausbreitung der Expansion, unterbindet aber die Ausbildung von Stoßfronten an den Schlitzenden. So wird die Überlagerung der aufgenommenen Spektren verschiedener Expansionszonen durch komplexe, an den Kanten entstehende Stoßwellenmuster ${ }^{28,35}$ verhindert. Querschnitte durch den Expansionsraum zeigen die Abbildungen $3.10 \mathrm{~d}$ und e. Die spezielle Fenstergeometrie (f) erlaubt eine Fixierung am Düsenkörper, die einen Gasfluss zwischen Düsenmaterial und Fenster minimiert und eine Durchsicht durch die Schlitzdüse ermöglicht. Dies vereinfacht einerseits die Justierung und lässt andererseits Untersuchungen der Expansion noch vor Austritt aus der Schlitzdüse zu. Aufgrund der insgesamt kleinen Anzahl von Einzeldetektoren, die für die Spektrenanlyse innerhalb des Schlitzes herangezogen werden können, des Bildrauschens durch den Metallkörper der Düse und des geringen Signal-zu-RauschenVerhältnisses an den Kantenregionen werden Standardmessungen so durchgeführt, dass sich der Düsenkörper gerade außerhalb des betrachteten Bilds befindet (weißer Rahmen in Abbildung $3.10 \mathrm{~b}$ ). 


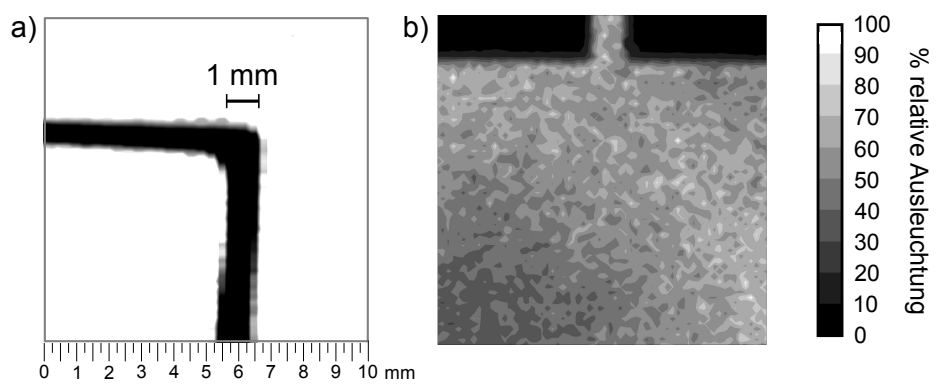

Abbildung 3.11: Charakterisierung des durch den FPA abgebildeten Bereichs an der Düsenposition. Dargestellt ist jeweils das Integral über das Einkanalspektrum von 3000$1000 \mathrm{~cm}^{-1}$ als Maß für die Lichtintensität. a) Metallwinkel mit definierter Materialdicke zur Abschätzung der Kantenlängen der abgebildeten Fläche, kontrastreiche Darstellung. b) Schlitzdüsenausgang (oben) mit evakuiertem Volumen dahinter, die seitliche Skala gibt die relative Lichtintensität an.

Der Abstand der Düse zum Parabolspiegel im Spektrometer ist so eingestellt, dass eine Fokussierung des zuvor parallelen Infrarotstrahls mit einem Durchmesser von etwa $4 \mathrm{~cm}$ um einen Faktor vier an der Position des Düsenausgangs erfolgt. Der FPA-Detektor mit Objektiv ist so positioniert, dass das gesamte Licht erfasst werden sollte und der geprobte quadratische Bereich somit eine Kantenlänge von etwa einem Zentimeter aufweisen müsste. Eine Überprüfung dieser Maße ist anhand eines Körpers bekannter Breite möglich. Abbildung 3.11 a zeigt ein FPA-Bild mit einem an der Düsenposition eingebrachten Metallwinkel. Die Darstellung wurde durch Integration über das Einkanalspektrum von $3000-1000 \mathrm{~cm}^{-1}$ zur Visualisierung der Lichtabschwächung durch den Probekörper und durch Maximierung des Bildkontrasts erhalten. Ausgehend von einer Materialdicke von $1 \mathrm{~mm}$ wird eine Kantenlänge des erfassten Bilds von $10 \mathrm{~mm}$ bestätigt. Gleichzeitig wird ersichtlich, dass die maximale räumliche Auflösung etwa $0.3 \mathrm{~mm}$ beträgt. Die Unterschiede in der Ausleuchtung des betrachteten Bereichs der Expansion direkt hinter dem Schlitzdüsenausgang zeigt Abbildung 3.11 b. Dargestellt ist erneut das Integral über die Einkanalspektren als Maß für die Gesamtlichtmenge pro Pixel. Hierbei zeigt sich eine Variation der Lichtintensität im Expansionsraum um mindestens einen Faktor zwei, die sich entsprechend auch in unterschiedlichen Signalzu-Rauschen-Verhältnissen widerspiegeln wird.

\subsubsection{Datenaufnahme}

Aufgrund der gegenüber Muesli- und Popcorn-Jet-Messungen längeren Messzeiten und der deutlich größeren generierten Datenmengen weicht der Messablauf mit dem ChilliJet etwas von der üblichen Methode ab.

Da zwischen den einzelnen Messtagen geringfügige Verschiebungen in der Düsenposition auftreten können, die sich aufgrund der räumlichen Auflösung direkt in den gemessenen Spektrensätzen niederschlagen, wird vor Beginn der Messungen die Detek- 
torhalterung so justiert, dass bei dem aufgenommenen Bild die Schlitzdüse möglichst exakt mittig positioniert ist und sich gerade außerhalb des gemessenen Bilds befindet. Da jeder der 4096 Einzeldetektoren einen eigenen Signalwert besitzt, wird eine geeignete Kombination von Spektralfilter, Abschwächerlagen und Offseteinstellung gewählt, die eine ausreichende Ausleuchtung für die meisten Pixel gewährleistet, ohne jedoch zu einer Übersättigung einzelner Detektoren zu führen.

Aufgrund der großen anfallenden Datenmengen ist bei FPA-Messungen die Spiegelgeschwindigkeit des Interferometers durch die Auslesegeschwindigkeit des Detektors begrenzt. Hieraus ergeben sich geringe Spiegelgeschwindigkeiten von $5 \mathrm{kHz}$ (gegenüber mindestens $80 \mathrm{kHz}$ bei Einzelelementdetektormessungen) und entsprechend längere Scanzeiten. Bei großen Druckverhältnissen $\frac{p_{0}}{p_{\mathrm{BG}}}$ erfolgen die Messungen mit gepulsten Expansionen, für geringe Druckverhältnisse werden kontinuierliche Expansionen und die unterbrechungsfreie Aufnahme einer ganzen Serie von Probespektren bevorzugt.

Messungen gepulster Expansionen werden über das Messmakro FPACYCLEMEASvar.mtx (siehe Anhang A.7) gesteuert. Die Pulsinitiierung erfolgt nicht über eine trsMethode, sondern direkt über die Ansteuerung des Pulsgenerators durch das Makro. Daher können sich Abweichungen des Scanbeginns in Abhängigkeit von der aktuellen Spiegelposition ergeben, wodurch eine Verlängerung der Gaspulse notwendig wird, um garantiert den gesamten Probescan abzudecken. Aufgrund dieser Totzeiten, in denen Expansionen ohne Messung erfolgen, wird die Effizienz durch Verwendung der Messmodi „double sided, forward backward“ und „,single sided, forward backward“ mit einer Aufnahme von vier bzw. zwei Spektren pro Scan gesteigert. Die resultierenden Pulsdauern von bis zu $6 \mathrm{~s}$ können in Abhängigkeit von den Messparametern dem Anhang A.6.2 entnommen werden. Die relativ langen Messzeiten aufgrund der niedrigen Spiegelgeschwindigkeiten verhindern auch eine sinnvolle Aufnahme von Nachscans, so dass ein Messzyklus nur aus zehn Hintergrundscans und einem Probescan besteht. Gleichzeitig werden so die erhaltenen Datenmengen reduziert. Zusammengehörige Hintergrund- und Probemessungen werden mit der gleichen Kennnummer abgespeichert. Die SpektrenMittelung und Fourier-Transformation nehmen deutlich mehr Zeit in Anspruch als bei Einzelelementdetektormessungen und erfolgen daher nicht automatisch im Anschluss an die Messungen.

\subsubsection{Datennachbehandlung}

Die zeitintensivste Komponente bei der Nachbehandlung der Chilli-Jet-Rohspektren ist die Fourier-Transformation. Daher bietet sich eine vorausgehende Mittelung der Hintergrund- und Probenspektren an. Dies wird allerdings durch das häufige Auftreten von Einzelscans mit abweichenden Centerburstpositionen der Interferogramme verhindert, die einen Anteil von bis zu 50\% an der gesamten Scanzahl ausmachen. Werden diese Scans in eine Mittelung einbezogen, so ergeben sich Interferogramme mit mehreren Peaks, die intensive periodische Störungen in den resultierenden Spektren hervorrufen. Um alle gemessenen Spektren verwerten zu können und gleichzeitig eine zeiteffiziente 

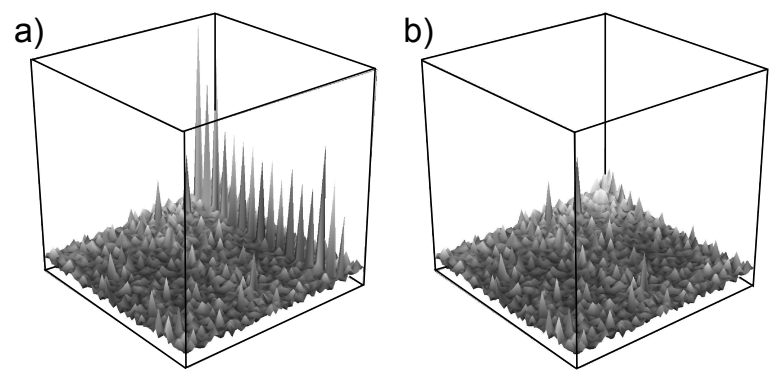

Abbildung 3.12: Quadratisches Rauschmittel von $1275-1100 \mathrm{~cm}^{-1}$ für eine Aufnahme des evakuierten Expansionsvolumens vor (a) und nach (b) dem Ersetzen der insgesamt 16 Spektren defekter Einzeldetektoren durch die ihrer Nachbarpixel.

Auswertung vorzunehmen, wird für die Spektrennachbehandlung das Auswertemakro chilliFT.mtx verwendet. Dieses separiert Spektren mit typischen und abweichenden Peakpositionen des Interferogramms und führt im ersten Fall die Mittelung vor der Fourier-Transformation aus, im zweiten Fall ist die Reihenfolge umgekehrt. Die zwei getrennt erhaltenen Resultatspektren werden dann mit ihrer Scanzahl gewichtet gemittelt.

Eine Visualisierung der Expansionen kann beispielsweise durch eine Integration von Spektralbereichen oder durch Spektrenanalyseparameter wie Peakpositionen oder das Signal-zu-Rauschen-Verhältnis erfolgen. Durch die Zuordnung des so für jeden Pixel individuell bestimmten Werts zu seiner räumlichen Position ergibt sich eine Eigenschaftskarte des geprobten Bereichs.

Aufgrund der geringen Pixelfläche ist das Signal-zu-Rauschen-Verhältnis für Einzelspektren grundsätzlich gering. Darüber hinaus existieren bei dem verwendeten FPA einige Pixel, die sich durch ein extrem hohes Rauschlevel auszeichnen. Abbildung 3.12 a zeigt eine Analyse des quadratischen Rauschmittels bei Aufnahme eines evakuierten Expansionsvolumens. Auffallend ist insbesondere eine Pixelreihe, in der jeder vierte Einzeldetektor ein extrem hohes Rauschlevel aufweist. Die Positionen dieser offenbar defekten Detektoren sind für jedes aufgenommene Spektrum identisch. Daher wird für die betreffenden Pixelnummern grundsätzlich ein Ersatz der generierten Spektren durch die der Nachbarpixel vorgenommen. Die Auswirkung dieses Vorgehens auf das Rauschlevel zeigt Abbildung 3.12 b.

Bei einer Gleichartigkeit aller (nicht defekten) Einzeldetektoren sollte sich in Abbildung 3.12 b eine ebene Fläche ergeben. Tatsächlich zeigt sich eine Heterogenität der Pixel. Aus den unterschiedlichen Rauschleveln und der ungleichmäßigen Ausleuchtung ergibt sich für alle Formen der einer Visualisierung zu Grunde liegenden Auswertungen ein Bildrauschen. Um dieses zu reduzieren und so reale Effekte leichter von Bildartefakten unterscheiden zu können, wurde daher zunächst eine Gruppierung von jeweils $2 \times 2$ Spektren vorgenommen, es wurden also je vier Spektren gemittelt und so ein auf noch $32 \times 32$ Pixeln basierendes Bild erhalten. Eine Veranschaulichung dieser Gruppierung 


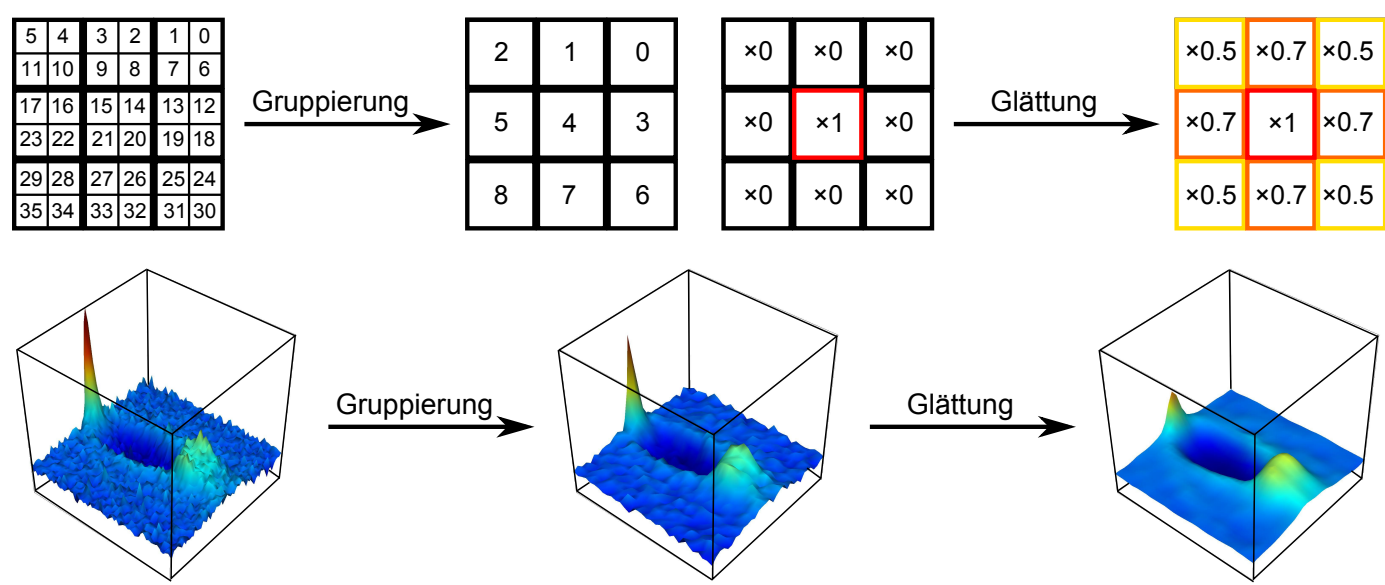

Abbildung 3.13: Nachbearbeitungsverfahren zur Verringerung des Bildrauschens: Mathematisches Vorgehen (oben) und Auswirkung auf die erhaltenen ortsaufgelösten Datenvisualisierungen (unten). Als Beispiel wurde das Integral über die $\nu_{3}$-Bande von Lachgas bei einer gepulsten Expansion von $5 \% \mathrm{~N}_{2} \mathrm{O}$ in Helium $\left(p_{0}=380 \mathrm{mbar}\right.$, $p_{\mathrm{BG}}=10$ mbar) verwendet.

und der Effekt auf die Expansionsbilder ist Abbildung 3.13 dargestellt. Die dreidimensionalen Datenwürfel wurden durch Expansion von 5\% Lachgas in Helium bei erhöhtem Hintergrunddruck und Integration über die $\nu_{3}$-Bande des $\mathrm{N}_{2} \mathrm{O}$ erhalten. Die Verringerung des Bildrauschens durch die Pixel-Gruppierung wird von einer Reduktion hoher Gradienten innerhalb des Bilds begleitet. In einem zweiten Schritt wird eine Glättung der Bildoberflächen durch ein Verfahren erreicht, das einem Gaußschen Weichzeichnereffekt ähnelt. Hierbei wird das Absorptionsspektrum jedes Pixels gewichtet mit seinen acht Nachbarpixeln gemittelt, wobei das zentrale Element den Gewichtungsfaktor 1, die vier an den Kanten angrenzenden Pixel den Faktor 0.7 und die vier an den Ecken liegenden Pixel den Faktor 0.5 erhalten. Die Mittelung erfolgt nur für Elemente, für die acht Nachbarpixel existieren. Daher wird der äußere Pixelring bei der Glättung abgeschnitten und die Gesamtdatenmenge verringert sich auf $30 \times 30$ Spektren. Wie Abbildung 3.13 zeigt, führt dieses Verfahren zu einer deutlichen Bildglättung, die eine klarere Unterscheidung zwischen realen und artifiziellen Strukturen der Expansion ermöglicht.

\subsubsection{Charakterisierung des pixelübergreifenden Rauschens}

Wie bereits in Abschnitt 2.5 erläutert, treten bei Focal Plane Array Detektoren verschiedene zusätzliche Rauschquellen auf, die beim Einsatz von Einzelelementdetektoren nicht bestehen. Werden in ihren Datenaufnahmeparametern vergleichbare Messungen mit einem $2 \mathrm{~mm}$ MCT-Detektor und dem MCT-FPA-Detektor durchgeführt, wobei für den FPA ein Mittelwertspektrum aller 4096 Pixel generiert wird und beide Detektoren an der gleichen Position relativ zum Spektrometer platziert werden, zeigt die FPA- 


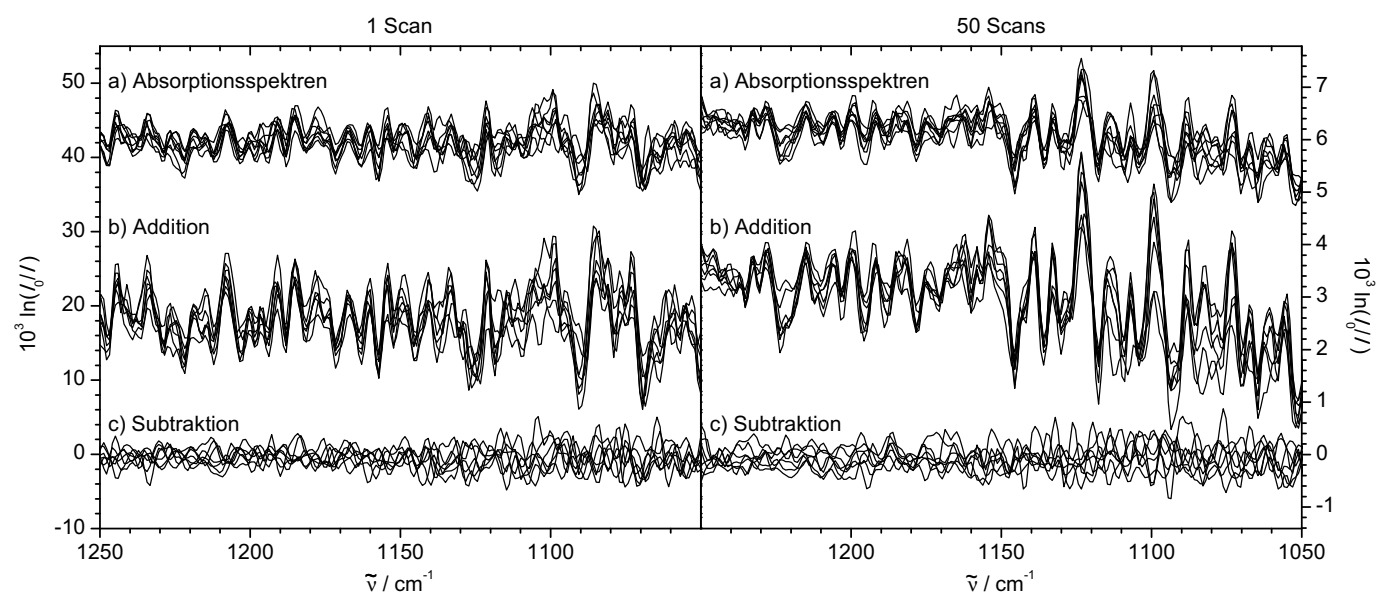

Abbildung 3.14: Rauschmuster a) von Absorptionsspektren, b) der Summe, c) der Differenz von Absorptionsspektren nebeneinanderliegender Pixel. Gezeigt sind die auf einer Bilddiagonalen liegenden Spektren von Leermessungen mit einem (links) und fünfzig (rechts) Scans nach Mittelung von jeweils $8 \times 8$ Spektren. Die Ordinatenachse der 50 Scan-Spektren ist um einen Faktor $\sqrt{50}$ vergrößert dargestellt.

Messung ein um etwa einen Faktor 50 stärkeres Rauschen trotz ähnlicher Gesamtdetektorflächen.

Abbildung 3.14 zeigt unterschiedlich behandelte Spektren aus FPA-Leermessungen mit einem (links) und fünfzig (rechts) Scans. Dargestellt sind die auf der Bilddiagonalen liegenden Spektren nach einer Gruppierung von jeweils $8 \times 8$ Pixeln. Diese Mittelung von je 64 Spektren wurde durchgeführt um eine Überlagerung des aus den geringen Einzeldetektorflächen resultierenden hohen Rauschens mit den FPA-spezifischen Effekten zu verringern. Die Intensitätsachse der 50-Scan-Spektren wurde dabei um einen Faktor $\sqrt{50}$ vergrößert abgebildet, so dass bei einer theoretischen Rauschreduktion durch Scanaddition mit der Wurzel der Scanzahl ${ }^{38}$ die dargestellten Absolutrauschintensitäten gleich sein sollten.

Die Spuren a) zeigen die übereinandergelegten Absorptionsspektren der Bilddiagonalen. Hier fallen zwei Charakteristika auf: Zum einen ist eine weitgehende Übereinstimmung der Rauschmuster der Diagonalspektren zu erkennen. Diese tritt nicht nur für einzelne Scans auf, sondern kann bei allen Spektren jeder Datenaufnahme beobachtet werden und ist auf eine mangelnde Separierung der Signale einzelner Detektoren im Auslese- und Datenübertragungsprozess zurückzuführen. Gleichzeitig nimmt die Rauschintensität etwas schwächer als mit der Wurzel der Scanzahl ab, so dass eine Reduktion dieser Rauschartefakte weder durch die Mittelung über mehrere Pixel noch vollständig durch Addition mehrerer Messungen möglich ist.

Die Abweichung des Rauschmusters verschiedener Pixel von einer statistischen Verteilung kann gut visualisiert werden, indem zwei Spektren nebeneinanderliegender Einzeldetektoren einmal addiert und einmal subtrahiert werden. Bei ideal statistischem 
Rauschen sollten beide Verfahren zu gleichen Rauschintensitäten führen. Die deutliche Abweichung hiervon zeigen die Spuren b) und c) in Abbildung 3.14. Während sich bei der Addition (b) Spektren mit stark übereinstimmendem Rauschen hoher Amplitude ergeben, zeigen sich nach der Subtraktion (c) eher statistische Rauschmuster bei einer gleichzeitig um etwa eine Größenordnung geringeren Intensität als bei der Addition. Nach Subtraktion ist die Rauschintensität gegenüber einer vergleichbaren Messung mit einem MCT-Einzelelementdetektor nur noch um etwa einen Faktor fünf erhöht. Die Diskrepanz zwischen Additions- und Subtraktionsergebnissen veranschaulicht das Ausmaß des nicht-statistischen Rauschens und macht weiterhin deutlich, dass die in Abschnitt 3.4.5 vorgestellten Methoden zur Bildglättung nicht maßgeblich zur Rauschreduktion der Spektren beitragen können, da sie im Wesentlichen auf der Addition von Spektren benachbarter Pixel basieren. Zielführender hinsichtlich der Spektrenqualität wären Subtraktionsverfahren, die allerdings auch immer eine Reduktion von Signalintensitäten bewirken. In Einzelfällen, bei denen Zonen des untersuchten Bereichs nahezu unbeeinflusst durch die Expansion und hierin befindliche absorbierende Substanzen sind (beispielsweise Regionen jenseits der lateralen Stoßfronten), kann jedoch die Subtraktion eines aus den substanzfreien oder -armen Spektren gewonnenen Mittelwertspektrums zu einer Verbesserung des Signal-zu-Rauschen-Verhältnisses der Spektren aus den Expansionsregionen führen. Hierbei muss jedoch grundsätzlich eine Abwägung des Kosten-Nutzen-Verhältnisses von Rausch- und Signalreduktion erfolgen. 


\section{Aggregationsverhalten in Multischlitzdïsenexpansionen}

Die Charakterisierung der Multischlitzdüsenexpansionen in Kapitel 3.2.4 legt zwei Anwendungsgebiete nahe. Zum einen kann die Erwärmung der Expansionen durch Kollisionszonen zur Beobachtung des Einflusses der Temperatur auf die Struktur kleiner Molekülaggregate genutzt werden. Zum anderen eröffnet die Möglichkeit der getrennten Zufuhr zweier Komponenten, deren Reaktivität eine Vormischung nicht zulässt, den Zugang zur Untersuchung der Mischclusterbildung solcher Systeme.

Nach einer Betrachtung der synchronen Expansion zweier Alkohole als Modellsystem für Wasser in Abschnitt 4.1 wird in Kapitel 4.2 zunächst der Einfluss thermischer Anregung auf die Struktur kleiner Wassercluster analysiert bevor in Abschnitt 4.3 dann die Bildung von Mischaggregaten bei der synchronen Expansionen der Isotopologe Wasser und Deuteriumoxid untersucht wird.

\subsection{Ein Modellsystem - Methanol und tert-Butylalkohol}

Als omnipräsentes Molekül steht Wasser im Fokus dieser Arbeit. Bereits die Analyse des Schwingungsspektrums des Monomers ist jedoch durch die komplexe Rotationsstruktur eines leichten asymmetrischen Rotors ${ }^{111}$ und die Kopplung der Normalmoden aufgrund von Fermi-Resonanzen und Coriolis-Wechselwirkungen ${ }^{112}$ problematisch. Die Aggregate des Wassers sind wegen der jeweils zwei potentiellen Wasserstoffbrückendonor- und -akzeptorstellen pro Molekül durch große, dreidimensionale Netzwerkstrukturen ${ }^{113}$ geprägt, wodurch die Untersuchung kleiner Cluster erschwert wird.

Alkohole stellen formal alkylierte Wassermoleküle dar, die jedoch aufgrund der fehlenden zweiten $\mathrm{O}-\mathrm{H}-\mathrm{Bindung}$ bevorzugt kleinere ketten- und ringförmige Aggregate ausbilden. ${ }^{3,114}$ Die Strukturen und Bindungsgeometrien kleiner Cluster niederer aliphatischer Alkohole ähneln denen des Wassers stark, wobei jedoch bei allen Vergleichen Effekte durch Wechselwirkungen der hydrophoben Alkylreste ${ }^{3}$ und die Unterschiede in der Elektronendichte an den freien Elektronenpaaren ${ }^{114}$ berücksichtigt werden müssen.

Soll die Ausbildung von Alkohol-Mischclustern als Modellsystem für die Aggregation von Wasser im Muesli-Jet untersucht werden, so ist dafür zunächst die Wahl zwei verschiedener, kleiner, aliphatischer Alkohole mit ausreichendem Dampfdruck notwen- 
dig, deren Spektren möglichst keine zusätzliche Komplexität durch das Auftreten von Torsionsisomeren aufweisen. Daher liegt die Verwendung von Methanol (MeOH) als kleinstem aller Alkohole und von tert-Butylalkohol ( $t \mathrm{BuOH})$ als nächst einfachem $\mathrm{Al}$ kohol mit nur einer Konformation nahe. Beide weisen jeweils nur eine strukturell einfache Dimerbande auf und sind daher gut für die Untersuchung des Heterodimers als kleinstem gemischten Cluster geeignet. Die Analyse von Trimerbanden hingegen soll an dieser Stelle unterbleiben, da hier bei beiden Alkoholen komplizierte Bandensysteme vorliegen, die im Fall von Methanol auf eine Überlagerung von Fundamentalen, heißen Banden sowie Summen- und Differenzbanden mit tiefliegenden Schwingungen zurückzuführen sind ${ }^{105}$ und beim tert-Butylalkohol vermutlich auf unterschiedliche Trimerstrukturen zurückgehen. ${ }^{115}$ Die Tetramerbanden, jeweils aufgrund der Ausbildung stabiler $\mathrm{S}_{4}$-symmetrischer Cyclen strukturell einfach, werden bereits durch die Banden größerer Aggregate überlagert. ${ }^{115,116}$

In der experimentellen Umsetzung wurde Methanol im Heliumstrom in den Sättiger eingebracht und auf Temperaturen zwischen -20 und $-30^{\circ} \mathrm{C}$ gekühlt. Um eine Überlagerung verschiedener Clusterbanden zu vermeiden, erfolgte bei dem Einfüllvorgang ins Reservoir eine zusätzliche Verdünnung mit Helium im Verhältnis $\mathrm{MeOH} / \mathrm{He}: \mathrm{He} \approx 1: 3$. Die Temperatur des tert-Butylalkohols betrug -15 bis $-20^{\circ} \mathrm{C}$, es wurde in der Form von Feststoffflocken eingefüllt. Obwohl ein möglichst rasches Ausfrieren und Einfüllen des tert-Butylalkohols angestrebt wurde, sind bei dieser Substanzpräparation leichte Verunreinigungen mit Wasser aus der Luftfeuchtigkeit nicht auszuschließen.

\subsubsection{Bildung gemischter Aggregate}

Entsteht durch Ausbildung einer Wasserstoffbrückenbindung zwischen zwei verschiedenen Alkoholmolekülen ein gemischtes Dimer, gibt es hierbei häufig eine energetische Präferenz bezüglich der Donor- und Akzeptorrolle. ${ }^{104,108}$ Ist der Energieunterschied zwischen den beiden möglichen Heterodimeren ausreichend hoch und gleichzeitig die Energiebarriere für eine Umwandlung zwischen den Isomeren ausreichend klein, kann bei einer Vormischung der Komponenten die (nahezu) exklusive Bildung eines Heterodimers in der Überschallexpansion beobachtet werden. ${ }^{104}$ Mithilfe des Multischlitzdüsenaufbaus können nun eventuelle Unterschiede bei getrennten und vorgemischten Expansionen sowie der Einfluss der Düsenplattengeometrie auf die gebildeten Aggregate untersucht werden.

Zunächst sollen die Clustereigenschaften bei der Multischlitzdüsenexpansion durch parallel angeordnete Schlitze betrachtet werden. Abbildung 4.1 a-e zeigt die Spektren der Expansionen der einzelnen Komponenten Methanol (a) und tert-Butylalkohol (b) durch jeweils jede zweite Schlitzdüse sowie der synchronen Expansion beider Substanzen in verschiedenen Abständen zur Düse (c, d) und der vorgemischten Alkohole (e). Die Konzentrationen der beiden Alkohole wurden dabei so eingestellt, dass in den Spektren der Einzelsubstanzen die Monomer- und Dimerbanden dominieren. Insbesondere bei Methanol konnten Beiträge durch höhere Aggregate wie dem Trimer (um $3470 \mathrm{~cm}^{-1}$ ) 


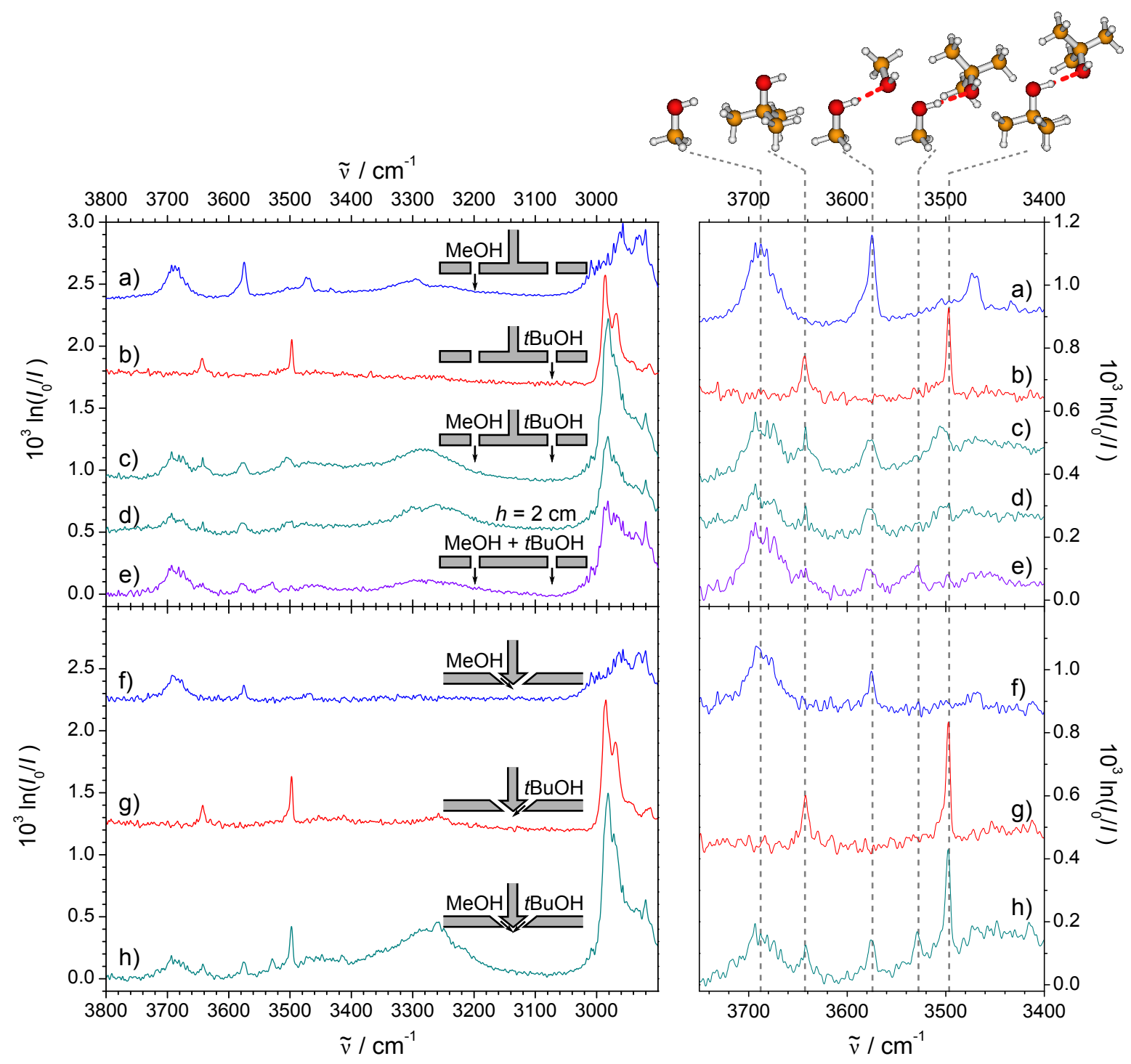

Abbildung 4.1: Expansionen von Methanol und tert-Butylalkohol durch die Multischlitzdüsen mit paralleler (a-e) und konvergierender $(\mathrm{f}-\mathrm{h})$ Schlitzanordnung, $p_{0}=500 \mathrm{mbar}$, $h=1 \mathrm{~cm}[\mathrm{~d}) h=2 \mathrm{~cm}] . \mathbf{a}), \mathbf{f}$ ) (blau): Expansion von Methanol in Helium durch eine Hälfte der Schlitzdüsen. b), g) (rot): Expansion von tert-Butylalkohol in Helium durch eine Hälfte der Schlitzdüsen. c), d), h) (grün): Synchrone Expansion von Methanol und tert-Butylalkohol durch jeweils jede zweite der Schlitzdüsen, d) bei erhöhtem Düsenabstand von $2 \mathrm{~cm}$. e) (violett): Expansion der vorgemischten Alkohole durch alle Schlitzdüsen. Links: Übersichtsspektrum. Rechts: Detailansicht des Bereichs der Monomer- und Dimer-O-H-Streckschwingungen mit Bandenpositionen aus Referenz 104 und berechneten Molekül- / Aggregatstrukturen ${ }^{117}$ (MP2/aug-cc-pVDZ). Banden um $3470 \mathrm{~cm}^{-1}$ sind auf Methanol-Trimere zurückzuführen. ${ }^{105}$ 


\begin{tabular}{ccccccc}
\hline \multirow{2}{*}{ Aggregat } & \multicolumn{2}{c}{ Experiment } & \multicolumn{2}{c}{ B3LYP } & \multicolumn{2}{c}{ MP2 } \\
& $\tilde{\nu} / \mathrm{cm}^{-1}$ & aus Ref. & $\tilde{\nu}^{\text {sk }} / \mathrm{cm}^{-1}$ & $D_{0} / \mathrm{kJ} \mathrm{mol}^{-1}$ & $\tilde{\nu}^{\mathrm{sk}} / \mathrm{cm}^{-1}$ & $D_{0} / \mathrm{kJ} \mathrm{mol}^{-1}$ \\
\hline \hline $\mathrm{MeOH}$ & 3686 & 104 & 3686 & & 3686 & \\
$(\mathrm{MeOH})_{2}$ & 3575 & 104 & 3536 & 18.4 & 3527 & 21.4 \\
$\mathrm{MeOD}$ & 2718 & 105 & 2718 & & 2718 & \\
$(\mathrm{MeOD})_{2}$ & 2638 & 105 & 2609 & 19.5 & 2601 & 22.4 \\
$t \mathrm{BuOH}$ & 3643 & 115 & 3643 & & 3643 & \\
$(t \mathrm{BuOH})_{2}$ & 3497 & 115 & 3491 & 16.9 & 3457 & 35.4 \\
$t \mathrm{BuOD}$ & 2688 & 118 & 2688 & & 2688 & \\
$(t \mathrm{BuOD})_{2}$ & 2584 & 118 & 2578 & 17.1 & 2553 & 36.2 \\
$\mathrm{MeOH}-t \mathrm{BuOH}$ & 3529 & 104 & 3513 & 19.5 & 3488 & 29.0 \\
$t \mathrm{BuOH}-\mathrm{MeOH}$ & & & 3510 & 16.4 & 3500 & 25.7 \\
$\mathrm{MeOD}-t \mathrm{BuOH}$ & 2607 & $*$ & 2592 & 20.4 & 2573 & 29.8 \\
$t \mathrm{BuOH}-\mathrm{MeOD}$ & & & 3512 & 16.4 & 3501 & 25.9 \\
\hline
\end{tabular}

Tabelle 4.1: Experimentelle (anharmonische) und rechnerische (skaliert harmonische) Wellenzahlen der intensivsten $\mathrm{O}-\mathrm{H}-$ bzw. O-D-Streckschwingungen sowie harmonisch nullpunktskorrigierte Dissoziationsenergien ausgewählter Methanol- und tert-Butylalkoholmonomere und -dimere. Bei gemischten Dimeren erfolgt die Angabe in der Form Donor-Akzeptor. Verwendete Rechenmethoden: ${ }^{117}$ B3LYP/6-311++G** MP2/augcc-pVDZ. *: aus dieser Arbeit. sk: Auf die Wellenzahl des Donor-Monomers skaliert. Die Werte der unskalierten Wellenzahlen, Infrarotintensitäten, Skalierungsfaktoren und Dissoziationsenergien $D_{\mathrm{e}}$ sind im Anhang A.4 tabelliert.

und dem Tetramer $\left(3294 \mathrm{~cm}^{-1}\right)$ zum Spektrum nicht vollständig vermieden werden. Die Bandenpositionen der intensivsten O-H-Streckschwingungen der Monomere und Dimere können Tabelle 4.1 entnommen werden.

Die Intensitäten der Alkyl-C-H-Streckschwingungen (um $3000 \mathrm{~cm}^{-1}$ ) sind relativ unabhängig vom Aggregationsgrad in der Expansion und können so als Maß für die Gesamtkonzentration der Alkohole herangezogen werden. Ein Vergleich der Spektren 4.1 a und b mit $4.1 \mathrm{c}$, die alle in einem Abstand von $1 \mathrm{~cm}$ zum Düsenausgang aufgenommen wurden, zeigt, dass im $\mathrm{C}-\mathrm{H}$-Bereich die Summe der Einzelkomponentenspektren das Spektrum der synchronen Expansion recht gut beschreibt. Im O-H-Bereich ist jedoch bei der Expansion beider Alkohole die verstärkte Bildung größerer Aggregate zu beobachten (Banden zwischen 3350 und $3200 \mathrm{~cm}^{-1}$ ), die auf die aus Kapitel 3.2 .4 bekannte Kompression der Expansionen bei verringertem Düsenabstand und die daraus resultierende höhere Stoßzahl zurückzuführen ist.

Die Ausschnittsvergrößerung der Spektren im Bereich der O-H-Streckschwingungen der Monomere und Dimere rechts in Abbildung 4.1 zeigt für das Spektrum c) der synchronen Expansion von Methanol und tert-Butylalkohol keine Banden, die nicht auch in den Spektren der Einzelkomponenten ( $\mathrm{a}$ und b) auftreten. Allerdings können deutliche Veränderungen bei den Banden der Homodimere beobachtet werden. Gegenüber dem Spektrum von reinem Methanol sinkt die Intensität der Methanol-Dimerbande trotz 
gleichbleibender Stagnationsbedingungen deutlich ab, während die Bande des tert-Butylalkoholdimers sich vor allem stark verbreitert und eine Blauverschiebung um $8 \mathrm{~cm}^{-1}$ gegenüber der Bandenposition von $3497 \mathrm{~cm}^{-1}$ aus Spektrum a) und der Literatur ${ }^{104,115}$ aufweist. Diese resultiert aus einer Aufweitung und Schwächung der Wasserstoffbrückenbindung durch thermische Anregung von OHO-Librationsschwingungen und anderen intermolekularen Moden. ${ }^{33}$ Während dies offenbar zu einer Dissoziation eines Teils der Methanoldimere führt, erkennbar an der abnehmenden Bandenintensität, wird das ohnehin stärker gebundene ${ }^{104}$ tert-Butylalkoholdimer vermutlich aufgrund von Dispersionswechselwirkungen zwischen den sperrigen Alkylresten zusätzlich stabilisiert. Dafür sprechen auch die in Tabelle 4.1 aufgeführten berechneten Dissoziationsenergien $D_{0}$ der Homodimere. Während die B3LYP-Rechnungen, bei denen energetische Beiträge durch Dispersionswechselwirkungen nicht beschrieben werden ${ }^{119}$, für das tert-Butylalkoholdimer einen kleineren Betrag der Dissoziationsenergie als für das des Methanols vorhersagen, ist der Wert aus der MP2-Methode, die Dispersion beinhaltet, etwa doppelt so groß. Ein Vergleich der Blauverschiebung um $8 \mathrm{~cm}^{-1}$ mit der Bandenposition in der Gasphase ${ }^{120}$ bei etwa $300 \mathrm{~K}$ von $3550 \mathrm{~cm}^{-1}$ weist unter der Annahme eines linearen Zusammenhangs zwischen Temperatur und Wellenzahl ${ }^{39}$ auf eine um etwa $40 \mathrm{~K}$ erhöhte Schwingungstemperatur aufgrund des Einflusses benachbarter Expansionen hin.

Auffällig ist zudem das vollständige Fehlen von Banden gemischter Dimere bei der synchronen Expansion von Methanol und tert-Butylalkohol, wie sie sowohl in Einfachschlitzdüsenexpansionen ${ }^{104,108}$ als auch bei der Multischlitzdüsenexpansion der vorgemischten Substanzen (Abbildung 4.1 e) bei einer Wellenzahl von $3529 \mathrm{~cm}^{-1}$ gefunden wurden. Auch eine verzögerte Bildung von Heterodimeren in der Expansion kann nicht beobachtet werden, da selbst bei einem erhöhten Düsenabstand von $2 \mathrm{~cm}$ (Spektrum 4.1 d) keine entsprechende Bande erkennbar ist. Diese Abwesenheit gemischter Cluster beim Einsatz von zwei Komponenten, deren Aggregatbildung barrierefrei verlaufen und energetisch gegenüber den Homodimeren keinen Nachteil aufweisen sollte (siehe Tabelle 4.1), ist ein klarer Hinweis auf eine ausbleibende Überkreuzung der benachbarten Expansionen. Offenbar findet eine Durchmischung der Substanzen höchstens in den wärmeren Zonen zwischen den Expansionen ${ }^{24}$ statt, die - wie anhand des Methanol-Dimers bereits deutlich wurde - eine Aggregation ohne zusätzliche stabilisierende Faktoren nicht zulassen. Dies bestätigt die Ausbildung einer Schichtstruktur von alternierenden Expansions- und Kompressionszonen. ${ }^{24}$

Auch bei einer Vormischung der Alkohole ist im Bereich der Dimer-O-H-Streckschwingungen, in Multi- wie in Einfachschlitzdüsenexpansionen, nur eine Bande zusätzlich zu denen der Reinsubstanzen erkennbar, obwohl theoretisch zwei gemischte Dimerstrukturen möglich wären. Die Ergebnisse der quantenchemischen Rechnungen in Tabelle 4.1 zeigen einen Unterschied der Dissoziationsenergien von etwas mehr als $3 \mathrm{~kJ} \mathrm{~mol}^{-1}$ mit einer Bevorzugung der Methanol-Donor-Struktur MeOH- $t \mathrm{BuOH}$, der gut mit weiteren B3LYP- und MP2-Rechnungen ${ }^{104}$ und Ergebnissen unter Verwendung des AMBER-Kraftfelds ${ }^{108}$ übereinstimmt. Messungen mit anderen Systemen haben gezeigt, dass eine Energiedifferenz dieser Größe durchaus zu einer vollständigen 


\begin{tabular}{lcccc}
\hline \multirow{2}{*}{ Alkohole } & \multicolumn{2}{c}{ B3LYP } & \multicolumn{2}{c}{ MP2 } \\
& $\boldsymbol{d}$ & $\boldsymbol{a}$ & $\boldsymbol{d}$ & $\boldsymbol{a}$ \\
\hline \hline $\mathrm{MeOH}+t \mathrm{BuOH}$ & 2.3 & -0.76 & -5.3 & -8.6 \\
$\mathrm{MeOD}+t \mathrm{BuOH}$ & 3.3 & -0.67 & -4.6 & -8.5 \\
\hline
\end{tabular}

Tabelle 4.2: Relative Donor- $(d)$ und Akzeptorstärken $(a)$ von Methanol bzw. D1-Methanol und tert-Butylalkohol in $\mathrm{kJ} \mathrm{mol}^{-1}$ gemäß Gleichungen 4.1 und 4.2 und basierend auf den Dissoziationsenergien $D_{0}$ aus Tabelle 4.1. Positive Werte entsprechen einer größeren Stärke von Methanol, negative von tert-Butylalkohol.

Präferenz eines der beiden Isomere führen kann (siehe auch Kapitel 5.1). ${ }^{121}$ Zusätzlich ergeben Rechnungen für eine Umwandlung zwischen den beiden gemischten Dimeren eine im Vergleich $\mathrm{zu}$ anderen Alkoholpaaren geringe Energiebarriere ${ }^{104}$ von nur etwa $3 \mathrm{~kJ} \mathrm{~mol}^{-1}$, die im Bereich hoher Stoßzahlen und Temperaturen am Beginn der Expansion durchaus überwunden werden kann. Eine Zuordnung der Mischclusterbande zu einer Heterodimerstruktur aufgrund der rechnerischen Wellenzahlen ist nicht möglich, da (wie Tabelle $4.1 \mathrm{zu}$ entnehmen ist) die gebundenen O-H-Streckschwingungsbanden beider möglicher Heterodimere in einem engen Wellenzahlfenster liegen und nach Skalierung auf das Donor-Monomer nicht einmal die relative Lage der Banden zueinander mit beiden verwendeten Methoden einheitlich vorhergesagt wird.

Die quantenchemischen Rechnungen treffen nicht nur Aussagen über die Energiedifferenzen zwischen den beiden Heterodimer-Isomeren, sie können auch Auskunft über relative Donor- und Akzeptorstärken geben. Für deren Berechnung existieren verschiedene Vorgehensweisen, die sich auf Energien, Bandenintensitäten oder Schwingungswellenzahlen stützen können. ${ }^{104,122}$ An dieser Stelle soll eine Berechnung aus den Dissoziationsenergien vorgenommen werden. Dabei wird die relative Donor- bzw. Akzeptorstärke $(d, a)$ der Alkohole aus den Dissoziationsenergien der Homo- und Heterodimere durch Berechnung des mittleren Energiegewinns oder -verlusts beim Austausch des Donorbzw. Akzeptormoleküls erhalten. (Notation: Donor-Akzeptor.)

$$
\begin{aligned}
& d=\frac{\left(D_{0}^{\mathrm{MeOH}-t \mathrm{BuOH}}-D_{0}^{t \mathrm{BuOH}-t \mathrm{BuOH}}\right)+\left(D_{0}^{\mathrm{MeOH}-\mathrm{MeOH}}-D_{0}^{t \mathrm{BuOH}-\mathrm{MeOH}}\right)}{2} \\
& a=\frac{\left(D_{0}^{\mathrm{MeOH}-\mathrm{MeOH}}-D_{0}^{\mathrm{MeOH}-t \mathrm{BuOH}}\right)+\left(D_{0}^{t \mathrm{BuOH}-\mathrm{MeOH}}-D_{0}^{t \mathrm{BuOH}-t \mathrm{BuOH}}\right)}{2}
\end{aligned}
$$

In dieser Formulierung stehen positive Werte von $d$ und $a$ für eine höhere Donor- bzw. Akzeptorstärke von Methanol, negative Werte hingegen für tert-Butylalkohol. Werden die nullpunktskorrigierten Dissoziationsenergien aus Tabelle 4.1 verwendet, ergeben sich die in Tabelle 4.2 aufgelisteten Werte der relativen Donor- und Akzeptorstärken. Demnach stellt auf Basis der B3LYP-Rechnungen Methanol den besseren Donor und tert-Butylalkohol den besseren Akzeptor dar, während auf MP2-Niveau tert-Butylalkohol bei beiden Werten dominiert, wobei der Betrag der relativen Akzeptorstärke der 
größere ist. Diese Diskrepanz zwischen den beiden Methoden wurde auch bei früheren Rechnungen gefunden ${ }^{104}$ und auf unterschiedliche Größen des Basissatzüberlagerungsfehlers sowie auf die fehlende Beschreibung von Dispersionswechselwirkungen durch die DFT-Methode zurückgeführt. Letztere erscheint hierbei besonders plausibel, da wie gezeigt bereits die Dissoziationsenergie des tert-Butylalkoholdimers durch die B3LYPRechnungen deutlich unterschätzt wird. Andererseits sind zumindest im Bezug auf die Vorhersage von Schwingungswellenzahlen des Methanol-Dimers die Unzulänglichkeiten harmonischer Rechnungen bekannt ${ }^{114}$, so dass auch hier eine gewisse Unsicherheit eingeht. Ungeachtet all dessen sagen beide Methoden eine Bevorzugung der Molekülorientierung mit Methanol als Donor im gemischten Dimer sowie einen Energiegewinn bei der Disproportionierung zweier Heterodimere mit tert-Butylalkohol als Donor zu zwei Homodimeren voraus.

Der experimentelle Nachweis dieser Donor-Präferenz wurde in Einfachschlitzdüsenexpansionen durch Einsatz von ${ }^{18} \mathrm{O}$-isotopenmarkiertem Methanol bereits erbracht ${ }^{104}$, wobei auch die Möglichkeit einer exakten Überlagerung von Banden beider Heterodimere ausgeschlossen wurde. Aufgrund der hohen Kosten für ${ }^{18} \mathrm{O}$-markierte Substanzen ist der alternative Einsatz des deuterierten Isotopologs eines Alkohols zur Aufklärung der Donor- und Akzeptorrollen lohnend. Bei einer Vormischung der Komponenten erfolgen dabei jedoch unerwünschte Isotopenaustauschreaktionen, durch die eine Strukturaufklärung durch Deuterierung verhindert wird. Entsprechend ist eine getrennte Substanzzufuhr bis zur Düse notwendig, wie sie im Multischlitzdüsenexperiment vorgesehen ist. Bei einer parallelen Anordnung der Schlitze lässt wie gezeigt jedoch die Ausbildung warmer Trennschichten zwischen den Expansionen die Bildung von Mischclustern nicht zu. Der Einsatz der konvergierenden Schlitzdüsen verhindert diese Trennschichten, indem die unterschiedlich zusammengesetzten Gasmischungen paarweise zu einer gemeinsamen Expansion gezwungen werden (siehe Kapitel 3.2). Die Expansionen der einzelnen Alkohole in Helium durch jeweils eine Hälfte der Schlitzdüsen in der konvergierenden Anordnung werden in den Spektren in Abbildung $4.1 \mathrm{f}$ und g gezeigt, das Spektrum bei synchroner Expansion der beiden Komponenten ist in Abbildung $4.1 \mathrm{~h}$ dargestellt. Die Expansionsbedingungen entsprechen bis auf die Düsengeometrie denjenigen der Spektren a) bis c). Wie bereits bei der Charakterisierung der Multischlitzdüsenexpansionen in Kapitel 3.2.4 gezeigt, ist bei der Verwendung nur jedes zweiten Schlitzes das Ausmaß an Clusterbildung mit der konvergierenden Anordnung geringer, wie hier auch insbesondere bei einem Vergleich der Spektren 4.1 a und f zu sehen ist. Bei der synchronen Expansion der beiden Alkohole, also bei Nutzung aller Düsenausgänge, zeigt sich für die konvergierende Anordnung gegenüber der parallelen einerseits eine insgesamt ausgeprägtere Aggregation und andererseits eine geringere Temperatur, die gut an der schmalen, nicht blauverschobenen Bande des tert-Butylalkoholdimers zu erkennen ist. Diese Unterschiede sind auf die größere Anzahl an Kollisionen zwischen den Molekülen bei den ineinanderströmenden Expansionen sowie den größeren Abstand der Schlitzdüsenpaare zueinander von $10 \mathrm{~mm}$ im Vergleich zum Abstand der parallelen Düsen von $5 \mathrm{~mm}$ zurückzuführen. Zusätzlich ist aber jetzt auch die Bande des Heterodimers bei 
$3529 \mathrm{~cm}^{-1}$ deutlich zu erkennen, die eine gemeinsame Expansion beider Alkohole ohne die Ausbildung einer Trennschicht innerhalb eines Expansionspaars beweist.

Die konvergierende Schlitzdüsenanordnung ist somit geeignet für die Untersuchung der Bildung gemischter Cluster. Dies beinhaltet jedoch nicht zwingend eine Eignung für die Expansion O-D isotopenmarkierter Substanzen, da ein Isotopenaustausch zu Beginn der Expansion bislang nicht ausgeschlossen werden kann.

\subsubsection{Isotopenaustausch}

Es ist also zu prüfen, ob die Aufklärung der Verteilung von Donor- und Akzeptorrolle im gemischten Methanol-tert-Butylalkohol-Dimer mit dem Muesli-Jet und der konvergierenden Schlitzdüsenanordnung durch Einsatz einer deuterierten Komponente möglich ist. Abbildung $4.2 \mathrm{e}-\mathrm{g}$ zeigt die Spektren der synchronen Expansion von monodeuteriertem Methanol $\left(\mathrm{CH}_{3} \mathrm{OD}, \mathrm{MeOD}\right)$ und tert-Butylalkohol $\left(\left(\mathrm{CH}_{3}\right)_{3} \mathrm{COH}\right.$, $t \mathrm{BuOH})$ mit steigendem Düsenabstand im Vergleich zu Spektren der reinen Alkohole (Abbildung $4.2 \mathrm{a}-\mathrm{d}$ ), jeweils mit $\mathrm{O}-\mathrm{H}-$ und O-D-Funktionalität. So kann ein eventueller Isotopenaustausch anhand der Positionen der Monomer- und (Homo-)Dimerbanden der Einzelkomponenten belegt werden. Gemäß MP2/aug-cc-pVDZ-Rechnungen ist dabei der Energiegewinn durch Deuterierung für die beiden Alkohole mit einer Differenz von weniger als $1 \mathrm{~kJ} \mathrm{~mol}^{-1}$ sehr ähnlich. Die Bandenposition der gebundenen O-H/DStreckschwingungsbande im Heterodimer ist im Wesentlichen vom Wasserstoffisotop an der Donorfunktionalität abhängig, während Unterschiede in der Hydroxylgruppe des Akzeptors beispielsweise bei Methanol nur eine Bandenverschiebung von etwa $2 \mathrm{~cm}^{-1}$ bewirken ${ }^{104}$, die somit in der Größenordnung der spektralen Auflösung liegt.

Das Spektrum der gleichzeitigen Expansion der beiden Alkohole in Abbildung 4.2 e zeigt keinerlei Anzeichen für einen Isotopenaustausch, der zur Bildung von MethanolOH- oder tert-Butylalkohol-OD-Monomeren oder Dimeren (blaue Bandenpositionen im $\mathrm{O}-\mathrm{H}-$ Bereich, rote im O-D-Bereich) führen würde. Auch die Bande des gemischten $\mathrm{MeOH}-t \mathrm{BuOH}-$ Dimers ist nicht erkennbar und erreicht somit zumindest keine Intensität oberhalb des Rauschlevels. Dafür zeigt sich eine zusätzliche Bande bei $2607 \mathrm{~cm}^{-1} \mathrm{im}$ Bereich der O-D-Streckschwingungen. Aufgrund der Lage zwischen den Homodimerbanden, der fehlenden Anzeichen für einen Isotopenaustausch und der sich ähnlich wie mit undeuteriertem Methanol verhaltenden relativen Donor- und Akzeptorstärken (siehe Tabelle 4.2), kann eine Zuordnung zum Dimer MeOD- $t \mathrm{BuOH}$ als sicher angesehen werden.

Um den Einfluss der Verweilzeit in der Expansion auf die beobachtete Unterdrückung des Isotopenaustauschs und die Bildung gemischter Aggregate zu untersuchen, wurden weitere Spektren der synchronen Expansion von Methanol und tert-Butylalkohol in größeren Abständen zum Düsenausgang aufgenommen (Abbildung $4.2 \mathrm{f}$ und $\mathrm{g}$ ). Ein Vergleich der relativen Bandenintensitäten von $(\mathrm{MeOD})_{2}$ und dem gemischten MeOD$t \mathrm{BuOH}-$ Dimer mit steigendem Abstand zeigt praktisch keine Veränderung des Intensitätsverhältnisses. Sollte also eine Verzögerung der Bildung gemischter Dimere gegen- 


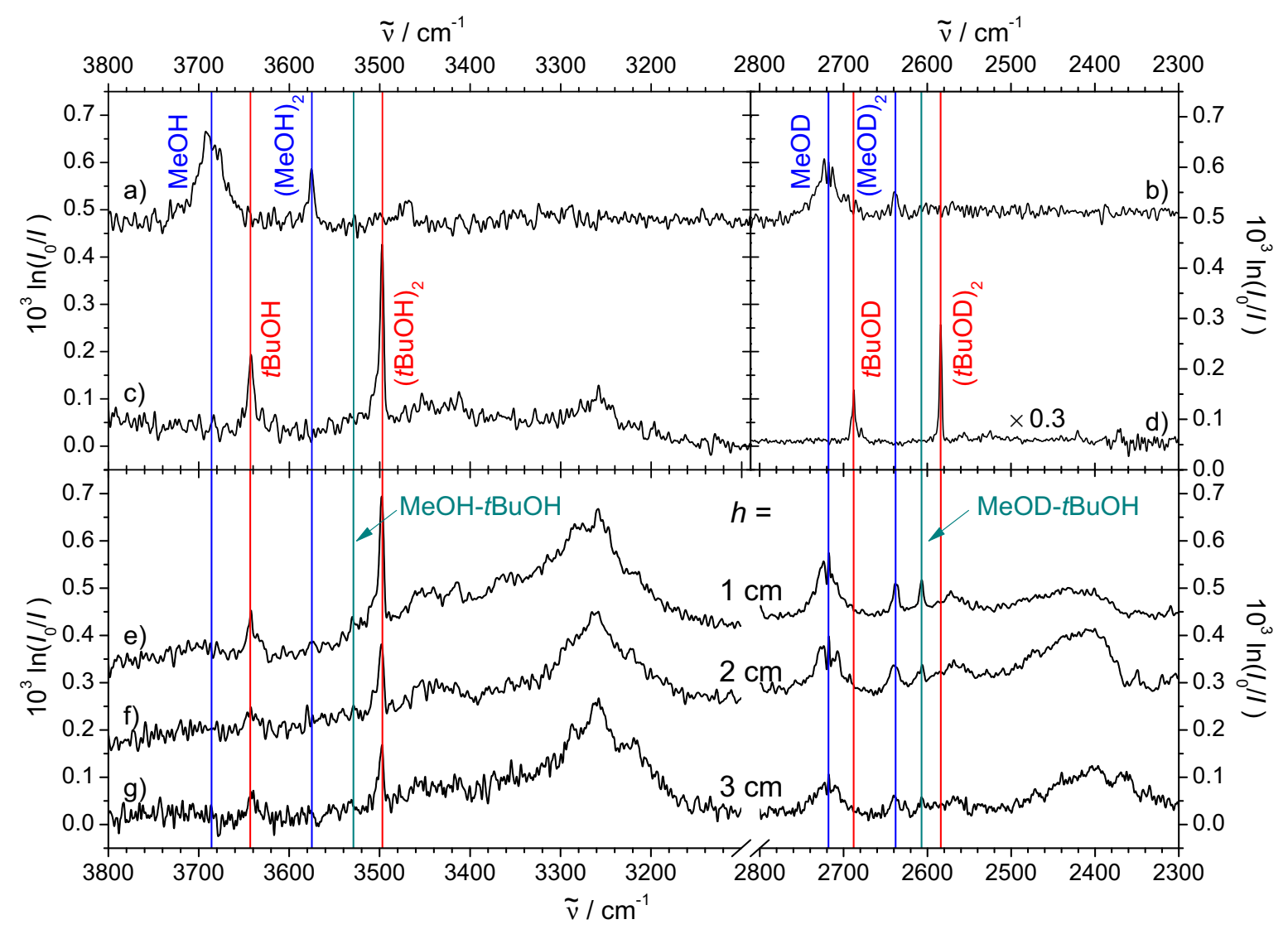

Abbildung 4.2: Expansion von teils isotopenmarkiertem Methanol und tert-Butylalkohol durch die Multischlitzdüse mit konvergierender Schlitzanordnung, $p_{0}=500 \mathrm{mbar}$, Trägergas Helium. a)-c) Expansionen durch jede zweite Schlitzdüse, $h=1 \mathrm{~cm}$. a) Methanol, b) Methanol-OD, c) tert-Butylalkohol; d) tert-Butylalkohol-OD (Spektrum zur Verfügung gestellt von Ass. Prof. René Wugt Larsen, keine Muesli-Jet-Expansion). e)-g) Synchrone Expansion von MeOD und $t \mathrm{BuOH}$ durch jeweils jede zweite der Schlitzdüsen bei verschiedenen Messabständen zum Düsenausgang. Die Bandenpositionen entsprechen denen aus Tabelle 4.1, Strukturen um $2350 \mathrm{~cm}^{-1}$ sind auf $\mathrm{CO}_{2}$ zurückzuführen. 
über den Homoaggregaten bestehen, tritt sie nur für sehr kleine Düsenabstände auf, die hier nicht ohne drastische Einbußen bei der Lichtintensität und somit dem Signalzu-Rauschen-Verhältnis untersucht werden können. Änderungen bei den drei betrachteten Abständen, die nicht auf den Dichteabfall in der Expansion zurückgeführt werden können, sind im Wesentlichen nur im Bereich der großen Cluster zwischen 3300 und $3200 \mathrm{~cm}^{-1}$ beziehungsweise 2450 und $2350 \mathrm{~cm}^{-1}$ zu erkennen. Während hier im $\mathrm{O}-\mathrm{H}-$ Bereich bei steigendem Abstand vor allem eine stärkere Strukturierung der Banden auftritt, erfolgt im O-D-Bereich eine Intensitätszunahme. Hier erschwert allerdings die Überlagerung mit der antisymmetrischen Streckschwingungsbande von $\mathrm{CO}_{2}$ um $2350 \mathrm{~cm}^{-1}$, das aus den Zwischenräumen zwischen Expansionsrohr und Spektrometer bzw. Detektorkammer stammen könnte, genauere Betrachtungen.

Die Intensitätszunahme im Bereich großer Cluster mit steigendem Abstand, die zwar im O-D-Bereich aber nicht im O-H-Bereich auftritt, kann durch die relativen Konzentrationen der beiden Alkohole in der Expansion erklärt werden. Aus dem Verhältnis der integrierten Monomerintensitäten $A$ aus Spektrum 4.2 e und den rechnerischen Bandenintensitäten $I$ auf MP2/aug-cc-pVDZ-Niveau ergibt sich das Anzahlverhältnis:

$$
\frac{n_{\mathrm{MeOD}}}{n_{t \mathrm{BuOH}}}=\frac{A_{\mathrm{MeOD}}}{A_{t \mathrm{BuOH}}} \cdot \frac{I_{t \mathrm{BuOH}}}{I_{\mathrm{MeOD}}} \approx 2.5 \cdot \frac{17 \frac{\mathrm{km}}{\mathrm{mol}}}{24 \frac{\mathrm{km}}{\mathrm{mol}}} \approx 1.8
$$

Durch die höhere Methanolkonzentration ist auch die Stoßdichte mit MeOD-Molekülen höher, so dass die Bildung methanolhaltiger Aggregate erst später in der Expansion abgeschlossen ist.

Bei den Banden größerer Aggregate im O-H-Bereich treten klar drei bis vier Banden mit Schwingungswellenzahlen von 3217, (3245,) 3259 und $3287 \mathrm{~cm}^{-1}$ hervor. Diese stimmen auch in ihrer Intensitätsverteilung recht gut mit den bei Expansionen von reinem tert-Butylalkohol gefundenen Banden bei 3213, 3247, 3259 und $3281 \mathrm{~cm}^{-1}$ über-

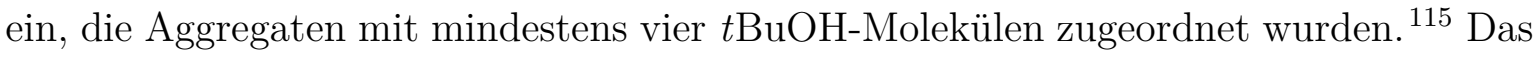
Hervortreten dieser Strukturierung erst bei größeren Düsenabständen kann einerseits auf die zunehmende Abkühlung und die daraus folgende sinkende Bandenbreite und die entsprechend bessere Separierung im Bereich der überlagerten Banden zurückgeführt werden. Andererseits wurden gerade die beiden seitlichen Banden bei 3217 und $3287 \mathrm{~cm}^{-1}$ Aggregaten mit mindestens fünf tert-Butylalkoholmolekülen zugeordnet ${ }^{115}$, so dass ihre späte Bildung in der Expansion auch anhand der Clustergröße begründet werden kann. Messungen bei Abständen zur Düse von mehr als $3 \mathrm{~cm}$ zur weiteren Verfolgung der Bandenentwicklung sind aufgrund des abnehmenden Signal-zu-RauschenVerhältnisses nicht sinnvoll.

Insgesamt kann mit dem Multischlitzdüsenaufbau und der konvergierenden Düsenanordnung also anhand der fehlenden Bande bei $3529 \mathrm{~cm}^{-1}$ und der zusätzlichen Bande bei $2607 \mathrm{~cm}^{-1}$ bei einer synchronen Expansion von tert-Butylalkohol und deuteriummarkiertem Methanol die Zusammensetzung des gemischten Dimers mit Methanol als Donormolekül der Wasserstoffbrückenbindung nachgewiesen werden. Interessant ist, 
dass selbst bei den gegenüber Einzelschlitzdüsenexpansionen höheren Temperaturen in der Multidüsenexpansion keine Anzeichen für die Bildung des energetisch benachteiligten Dimers mit Methanol als Akzeptor erhalten werden. Dies könnte jedoch der niedrigen Energiebarriere für die Isomerisierung zwischen den beiden Dimeren geschuldet sein. Die Monomer- und Dimerbanden weisen auf eine Unterdrückung des Isotopenaustauschs auch noch bei großen Abständen zur Düse hin. Nicht ausgeschlossen werden kann jedoch, dass bei gemischten cyclischen Aggregaten mit mindestens drei Alkoholmolekülen ein Isotopenaustausch durch Umlagerung im Ring erfolgt. Die daraus resultierenden neuen Streckschwingungsbanden wären aufgrund der komplexen Trimerbandenbereiche und der starken Bandenüberlagerung für große Cluster nicht identifizierbar. Gegen diesen Prozess spricht allerdings die zunehmende Strukturierung des Bereichs zwischen 3300 und $3200 \mathrm{~cm}^{-1}$ mit steigendem Düsenabstand. Für die Klärung der Frage, ob ein Isotopenaustausch in den Expansionen durch Umlagerung in cyclischen Aggregaten erfolgt, bietet sich das eigentlich kompliziertere System $\mathrm{H}_{2} \mathrm{O} / \mathrm{D}_{2} \mathrm{O}$ an. Ein Isotopenaustausch in Aggregaten beliebiger Größe führt hier unweigerlich zur Bildung von HDO, welches unabhängig von seiner Einbindung in Cluster eine von denen des $\mathrm{H}_{2} \mathrm{O}$ und $\mathrm{D}_{2} \mathrm{O}$ isolierte Bande der Knickschwingung besitzt. Synchrone Expansionen von Isotopologen des Wassers werden in Abschnitt 4.3 untersucht.

\subsection{Warme Expansionen - Wasser}

Wasser $\left(\mathrm{H}_{2} \mathrm{O}\right)$ gehört zu den häufigsten Verbindungen auf der Erde ${ }^{123}$ und ist zudem als das wichtigste Wasserstoffbrückenbindungen bildende Molekül anzusehen, da ohne deren Ausbildung Wasser bei Standardbedingungen nicht als Flüssigkeit vorläge und somit eine der wichtigsten Grundlagen für Leben auf unserem Planeten nicht gegeben wäre.

Die Gasphase wasserstoffbrückenbildender Moleküle enthält grundsätzlich Dimere und höhere Cluster ${ }^{124}$, deren Konzentrationen mit sinkender Temperatur stark ansteigen. ${ }^{125}$ Bei Systemen mit hierarchischen intermolekularen Wechselwirkungen sind diese Cluster überwiegend klein und strukturell einfach aufgebaut. Aufgrund des entropischen Nachteils bei der Nukleation wachsen sie bei steigender Substanzkonzentration oder sinkender Temperatur zunächst bis zu einer kritischen Aggregatgröße an, bevor dann eine spontane homogene Kondensation einsetzt. ${ }^{126}$ Dieser Prozess kann nicht nur für die im vorhergehenden Kapitel 4.1 betrachteten Alkohole, sondern auch beispielsweise für Fluorwasserstoff ${ }^{127}$ und Carbonsäuren ${ }^{128}$ beobachtet werden.

Für Wasser gestaltet sich die Untersuchung kleiner Aggregate in der Gasphase problematischer, da solche Ketten und Ringe aus wenigen Molekülen noch immer eine große Anzahl freier Wasserstoffbrückendonor- und -akzeptorfunktionalitäten aufweisen, die die Entstehung großer, dreidimensionaler Netzwerke ermöglichen und begünstigen. ${ }^{113}$ Der Energiegewinn durch die zusätzlichen Wasserstoffbrückenbindungen kompensiert dabei den Entropienachteil. Während das Gasphasenspektrum des Wasser-Monomers 


\begin{tabular}{lccc}
\hline & $\nu_{1}$ & $\nu_{2}$ & $\nu_{3}$ \\
\hline \hline Typ & symmetrische & Knick- & asymmetrische \\
Wellenzahl $^{142}$ & Streckschwingung & schwingung & Streckschwingung \\
Symmetrie & $3656.65 \mathrm{~cm}^{-1}$ & $1594.59 \mathrm{~cm}^{-1}$ & $3755.79 \mathrm{~cm}^{-1}$ \\
$\begin{array}{l}\text { Exp. Übergangs- } \\
\text { dipolmoment }\end{array}$ & $\mathrm{A}_{1}$ & $\mathrm{~A}_{1}$ & $\mathrm{~B}_{2}$ \\
$\begin{array}{l}\text { Integrierte } \\
\text { Bandenstärke }^{144}\end{array}$ & $-0.02170 \mathrm{D}$ & $0.17946 \mathrm{D}$ & $0.09714 \mathrm{D}$ \\
\hline
\end{tabular}

Tabelle 4.3: Schwingungseigenschaften von Wasser.

schon lange Gegenstand des Interesses ${ }^{129}$ und heute gut untersucht ist und auch die verschiedenen spektroskopischen Eigenschaften kleiner, kalter Wasseraggregate in Expansionen bekannt sind ${ }^{3,107,130,131,132}$, ist deren Nachweis unter atmosphärischen Bedingungen weitaus komplizierter.

Das Wasser-Monomer ist ein leichter, asymmetrischer Rotor, wodurch die charakteristischen Clusterbanden der gebundenen O-H-Streckschwingungen der Aggregate ${ }^{133}$ von einem breiten, komplexen Monomerspektrum überlagert werden. ${ }^{134}$ Informationen zu den Schwingungseigenschaften des Wasser-Monomers können Tabelle 4.3 entnommen werden. Zudem ist aufgrund der hohen Polarität von Wasser Absorption durch wechselwirkende Stoßpartner nur schwer von der gebundener oder zumindest metastabiler Dimere zu unterscheiden. ${ }^{2,135}$

Die Suche nach kleinen Aggregaten des Wassers in der Gasphase hat eine lange Tradition ${ }^{136}$ und ist durch das Auftreten von Wasserabsorptionen außerhalb der Bereiche der typischen Monomerschwingungen motiviert, die potentiell großen Einfluss auf das Strahlungsgleichgewicht der Erde besitzen. ${ }^{137,138}$ Neben gemischten Clustern von Wasser mit molekularem Stickstoff ${ }^{139}$ werden als Ursache hierbei vor allem kleine Wasseraggregate angenommen. ${ }^{2,135,140}$ Aus den genannten Gründen gestaltete sich dabei insbesondere der experimentelle Nachweis des atmosphärischen Wasser-Dimers schwierig, der trotz zahlreicher spektroskopischer Untersuchungen ${ }^{134}$ und Simulationen ${ }^{141}$ erst kürzlich und bei hohen Partialdrücken von 17 mbar durch Rotationsspektroskopie überzeugend gelang. 6

Die warmen Expansionen des Muesli-Jets könnten als Übergangssystem zwischen klassischen, kalten Expansionen und der Gasphase in der Lage sein eine Richtung für die schwingungsspektroskopische Suche nach atmosphärischen Aggregaten anzuzeigen. Dafür wurden durch systematische Reduktion der adiabatischen Kühlung schrittweise wärmere Expansionen von Wasser generiert. Ausgehend von klassischen Einfachschlitzdüsenexpansionen mit hohem Stagnationsdruck und Helium als Trägergas ${ }^{107}$ wurde in einem ersten Schritt die Düse gegen den Muesli-Jet Multischlitzdüsenaufbau mit paralleler Düsenanordnung ausgetauscht, wobei zunächst nur jede zweite Schlitzdüse 


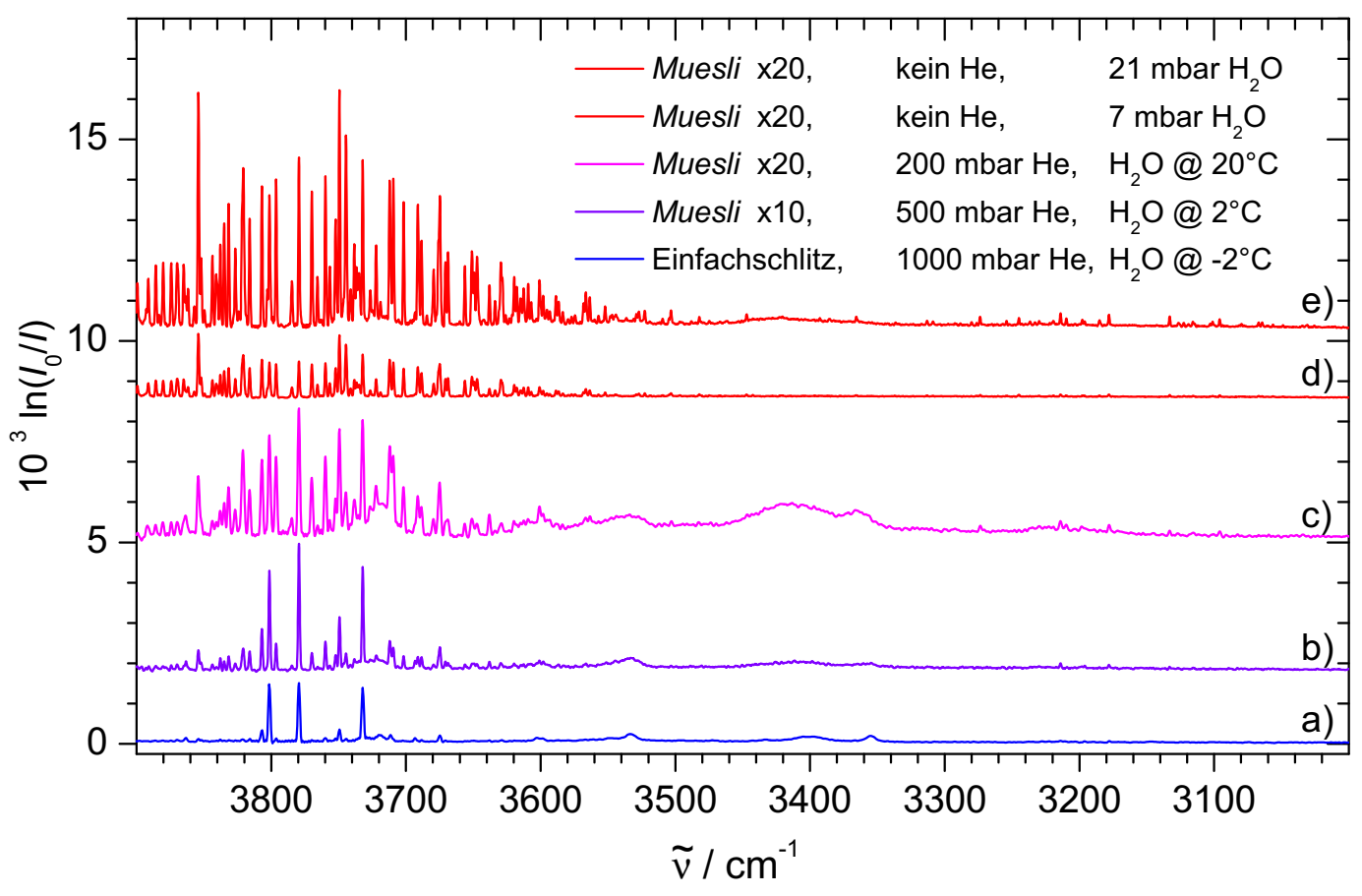

Abbildung 4.3: Übersichtsspektren über den gesamten $\mathrm{O}-\mathrm{H}-\mathrm{Streckschwingungsbereich} \mathrm{von}$ Wasser bei verschiedenen Expansionsbedingungen. a) Expansion durch Einfachschlitzdüse der Dimension $120 \times 0.5 \mathrm{~mm}^{2}$ bei hohem Stagnationsdruck, Spektrum wurde Referenz 107 entnommen. b) Expansion durch jede zweite Düse des Muesli-Jets mit paralleler Schlitzanordnung, reduzierter Stagnationsdruck. c) Expansion durch jede Düse des Muesli-Jets, weiter reduzierter Hintergrunddruck, hohe Wassertemperatur. d) Trägergasfreie Expansion durch jede Düse des Muesli-Jets, $\vartheta\left(\mathrm{H}_{2} \mathrm{O}\right)=25^{\circ} \mathrm{C}$, $p\left(\mathrm{H}_{2} \mathrm{O}\right)=7$ mbar. e) Wie d), $p\left(\mathrm{H}_{2} \mathrm{O}\right)=21$ mbar. Die Spektrenkennungen a)-e) werden für folgende Darstellungen beibehalten.

verwendet und durch eine Reduzierung des Stagnationsdrucks das Ausmaß an Kollisionskühlung reduziert wurde. Im nächsten Schritt wurde der Reservoirdruck weiter verringert und gleichzeitig alle 20 der parallelen Schlitzdüsen verwendet um den Einfluss der warmen Kollisionszonen auf die Expansion zu erhöhen. Schließlich wurde vollständig auf ein Trägergas verzichtet, so dass die Kondensationswärme bei der Aggregation nicht mehr effizient abgeführt werden kann und eine Erhöhung der Clustertemperatur erfolgt.

Abbildung 4.3 zeigt die O-H-Streckschwingungsspektren der Wasserexpansionen bei den verschiedenen experimentellen Bedingungen. Neben der sukzessiven Erhöhung der Expansionstemperatur wurden dabei mit Ausnahme von Spektrum c) möglichst geringe Beiträge durch Wasser-Hexamere ${ }^{5,145}$ angestrebt, da deren delokalisiertes Schwingungsspektrum die Auswertung unnötig erschweren würde. ${ }^{146}$ Spektrum 4.3 a wurde bei der Expansion von Wasser in Helium mit einem Stagnationsdruck von 1 bar und unter 
Einsatz einer Einfachschlitzdüse $\left(120 \times 0.5 \mathrm{~mm}^{2}\right)$ erhalten. Es wird dominiert von drei Monomerlinien der Übergänge $2_{02} \leftarrow 1_{01}\left(3801.5 \mathrm{~cm}^{-1}\right), 1_{01} \leftarrow 0_{00}\left(3779.4 \mathrm{~cm}^{-1}\right)$ und $0_{00} \leftarrow 1_{01}\left(3732.1 \mathrm{~cm}^{-1}\right)^{147}$ sowie relativ schmalen Clusterbanden. Beim Übergang auf jede zweite der 20 parallelen Muesli-Jet-Düsen und einer Halbierung des Stagnationsdrucks auf 500 mbar (Spektrum b) zeigt bereits der breitere Monomer-Bereich mit einer höheren Anzahl an Rotationsschwingungsbanden eine höhere Temperatur der Expansion an. Wird die Anzahl der Düsen verdoppelt, ihr Abstand zueinander somit halbiert und gleichzeitig der Stagnationsdruck auf 200 mbar abgesenkt und die Wassertemperatur erhöht (Spektrum c), findet eine weitere Verbreiterung des Monomerbereichs statt. Dazu sind nun Beiträge durch höhere Cluster wie dem Hexamer mit der charakteristischen Bande ${ }^{133}$ bei $3200 \mathrm{~cm}^{-1}$ zu erkennen, ebenso wie eine beginnende Überlappung der Monomerlinien mit den gebundenen $\mathrm{O}-\mathrm{H}-\mathrm{Streckschwingungsbanden} \mathrm{der} \mathrm{Aggregate.}$ Für eine Maximierung der Expansionstemperatur (Spektrum 4.3 e) wurde das Trägergas vollständig aus der Expansion entfernt, es wurden alle 20 der parallelen Schlitzdüsen verwendet, die Wassertemperatur im Sättiger mit $25^{\circ} \mathrm{C}$ soweit erhöht, dass gerade noch keine Kondensation in den Zuleitungen zum Reservoir erfolgte und der Stagnationsdruck mit 21 mbar so hoch gewählt, wie akzeptable Wartezeiten von $60 \mathrm{~s}$ zwischen zwei Gaspulsen es zuließen (zum Vergleich: $\left.p^{25^{\circ} \mathrm{C}}\left(\mathrm{H}_{2} \mathrm{O}\right)=31.7 \mathrm{mbar}\right)^{148}$. Neben dem nun sichtbaren Oberton der Knickschwingung des Wasser-Monomers mit einem Bandenzentrum um $3150 \mathrm{~cm}^{-1}$ ist hier vor allem eine ausgedehnte Überlappung zwischen dem Monomer-Streckschwingungsbereich und den Aggregatbanden zu erkennen. Aus diesem Grund wurde zusätzlich das Referenzspektrum $4.3 \mathrm{~d}$ mit einem niedrigeren Stagnationsdruck von 7 mbar und somit ohne signifikante Clusterbeiträge aufgenommen um durch skalierte Subtraktion der Spektren e) und d) ein Spektrum der warmen Aggregate mit möglichst geringen Beeinträchtigungen durch Monomerbanden erhalten zur können. Für die Aufnahme von Spektrum d) wurde infolge der kurzen Nachfüllzeit bis zum Erreichen des gewünschten Reservoirdrucks eine leicht abgewandelte trsMethode mit nur 10 anstelle der sonst eingesetzten 20 Hintergrundscans verwendet. Aufgrund der in diesen Übersichtsspektren bereits erkennbaren Auswirkungen der Expansionsbedingungen auf die Monomer- und Clusterbanden sollen nun eine indirekte Quantifizierung der Erwärmung anhand der Monomer-Rotationstemperatur und eine Analyse des Einflusses der Temperatur auf die Wasseraggregate vorgenommen werden.

\subsubsection{Monomer-Rotationstemperaturen}

Die Abschätzungen der mittleren Rotationstemperatur der Wasser-Monomere erfolgen aufgrund der komplexen Rotationsstruktur asymmetrischer Rotatoren anhand von Vergleichen der experimentellen Ergebnisse mit Spektren des Simulationsprogramms pgopher ${ }^{149}$ bei verschiedenen Temperaturen. Um einen Richtwert für die Verlässlichkeit der Simulationen zu erhalten, wird zunächst ein bei bekannter Temperatur von $300 \mathrm{~K}$ gemessenes Gasphasen-Wasserspektrum mit simulierten Spektren sowohl unter der Näherung des starren Rotators (also unter Vernachlässigung von Zentrifugaldeh- 


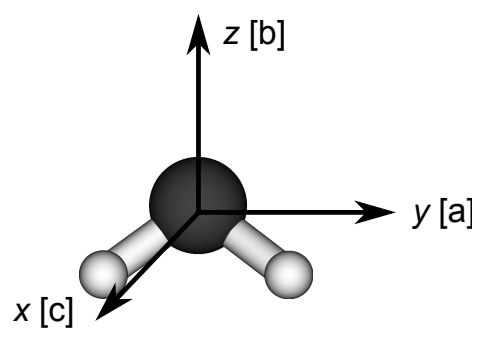

Abbildung 4.4: Wassermonomer mit eingezeichneten Koordinatenachsen (Ursprung im Molekülschwerpunkt). a, b, c: Entsprechen den Rotationsachsen der Rotationskonstanten A, B, C. $x, y, z$ : Koordinaten der pgopher-Konvention, die Achsenkombination entspricht der Molekülrepräsentation II $r$.

nungseffekten) als auch unter Verwendung aller zugänglicher Molekülparameter verglichen. Die für beide Simulationen verwendeten Werte können Tabelle 4.4 entnommen werden. Da durch das Programm pgopher eine Festlegung der $z$-Achse als $\mathrm{C}_{2}$-Symmetrieachse und der $x$-Achse als senkrecht zur Molekülebene erfolgt, ergibt sich zusammen mit der Konvention der Achsenbenennung „a, b, c" entsprechend der in absteigender Größe geordneten zugehörigen Rotationskonstanten $A, B$ und $C$ so die in Abbildung 4.4 gezeigte Achsenbenennung. Diese entspricht der Molekülrepräsentation II $r$.

Abbildung 4.5 a zeigt den Vergleich eines gemessenen Gasphasenspektrums von Wasser (oben) mit pgopher-Simulationen unter der Annahme eines starren Rotators (Mitte) und unter Einbeziehung der Zentrifugaldehnung (unten). Um nicht nur einen Vergleich der einzelnen Linien, sondern auch der gesamten Bandenform anstellen zu können, sind alle drei Spektren in Abbildung $4.5 \mathrm{~b}$ mit einer reduzierten Auflösung von $20 \mathrm{~cm}^{-1}$ beziehungsweise nach Faltung der Linienspektren mit Gaußprofilen der vollen Halbwertsbreite $20 \mathrm{~cm}^{-1}$ gezeigt. Insgesamt fällt hierbei zunächst auf, dass bei den höher aufgelösten Spektren zwar die allgemeine Breite des Bandensystems sowie einige Charakteristika wie die hohe Liniendichte um $3740 \mathrm{~cm}^{-1}$ und die Bandenlücke um $3790 \mathrm{~cm}^{-1}$ gut übereinstimmen. Allerdings ist im experimentellen Spektrum zum einen eine deutlich höhere Linienanzahl als in der Simulation zu erkennen und auch die Intensitätsverteilungen der Banden insbesondere zwischen 3800 und $3900 \mathrm{~cm}^{-1}$ zeigen deutliche Abweichungen. Es ist jedoch anhand der Intensitätsverteilung der P-Zweig-Banden zu erkennen, dass eine Einbeziehung der Zentrifugaldehnungskonstanten und des Herman-Wallis-Koeffizienten notwendig für eine zufriedenstellende Simulation des Wasser-Monomerspektrums sind, wie aufgrund der geringen Trägheitsmomente von Wasser auch zu erwarten ist. ${ }^{112}$ Die so erhaltene bessere Übereinstimmung zwischen Experiment und Simulation lässt sich besonders gut anhand der niedrig aufgelösten Spektren in Abbildung 4.5 b erkennen, wo nicht nur für kleine Wellenzahlen sondern auch für den R-Zweig die deutlich bessere Beschreibung des Intensitätsverlaufs durch den nicht-starren Rotator ersichtlich ist. Der entsprechende Parametersatz wird daher für alle folgenden Untersuchungen zur Monomer-Rotationstemperatur des Wassers eingesetzt. 


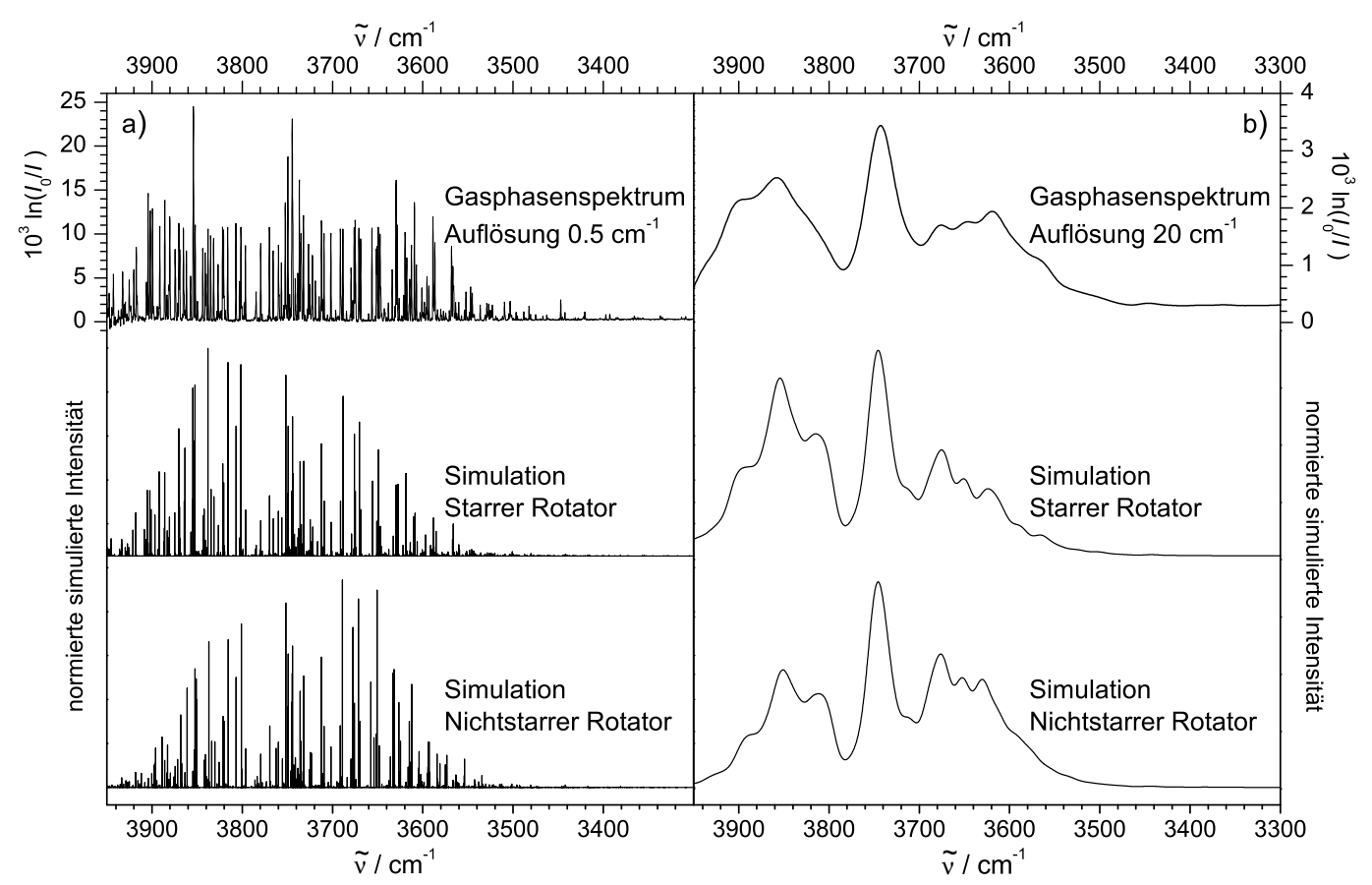

Abbildung 4.5: Gemessene und simulierte Gasphasenspektren von Wasserdampf. Die Spektrensimulation erfolgte mithilfe des Programms pgopher ${ }^{149}$ und den in Tabelle 4.4 aufgeführten Parametern. Simulationstemperatur $300 \mathrm{~K}$, die simulierten Spektren bestehen aus Gaußprofilen mit an die experimentellen Daten angepassten Halbwertsbreiten. Oben: Experimentelle Spektren. Mitte: Simulation eines starren Rotators. Unten: Simulation unter Einbeziehung von Zentrifugaldehnung. a) Experimentelle Auflösung $0.5 \mathrm{~cm}^{-1}$. b) Experimentelles Spektrum entspricht dem aus a) mit einer verringerten Auflösung von $20 \mathrm{~cm}^{-1}$. 


\begin{tabular}{|c|c|c|}
\hline Größe & Wert & aus Ref. \\
\hline \multicolumn{3}{|l|}{ Allgemeines } \\
\hline Spezies & Asymm. Kreisel & \\
\hline Punktgruppe & $\mathrm{C}_{2 \mathrm{v}}$ & \\
\hline $\mathrm{C}_{2}$-Achse & b & \\
\hline Out-of-plane-Achse & c & \\
\hline Repräsentation & $\mathrm{II} r$ & \\
\hline Stat. Gewicht Kernspins ee & 1 & 151 \\
\hline Stat. Gewicht Kernspins eo & 3 & 151 \\
\hline Stat. Gewicht Kernspins oe & 3 & 151 \\
\hline Stat. Gewicht Kernspins oo & 1 & 151 \\
\hline \multicolumn{3}{|l|}{ Grundzustand } \\
\hline Symmetrie & A1 & \\
\hline Rotationskonstante $A / \mathrm{cm}^{-1}$ & 27.8806172 & 152 \\
\hline Rotationskonstante $B / \mathrm{cm}^{-1}$ & 14.5216065 & 152 \\
\hline Rotationskonstante $C / \mathrm{cm}^{-1}$ & 9.27776779 & 152 \\
\hline Quart. Zentrifugaldehnungskonst. $\Delta_{K} / \mathrm{cm}^{-1}$ & $3.249738 \cdot 10^{-2}$ & 152 \\
\hline Quart. Zentrifugaldehnungskonst. $\Delta_{J K} / \mathrm{cm}^{-1}$ & $-5.782700 \cdot 10^{-3}$ & 152 \\
\hline Quart. Zentrifugaldehnungskonst. $\Delta_{J} / \mathrm{cm}^{-1}$ & $1.253627 \cdot 10^{-3}$ & 152 \\
\hline Quart. Zentrifugaldehnungskonst. $\delta_{K} / \mathrm{cm}^{-1}$ & $1.32245 \cdot 10^{-3}$ & 152 \\
\hline Quart. Zentrifugaldehnungskonst. $\delta_{J} / \mathrm{cm}^{-1}$ & $5.07668 \cdot 10^{-4}$ & 152 \\
\hline Sext. Zentrifugaldehnungskonst. $\mathrm{H}_{K} / \mathrm{cm}^{-1}$ & $1.28234 \cdot 10^{-4}$ & 152 \\
\hline Sext. Zentrifugaldehnungskonst. $\mathrm{H}_{K J} / \mathrm{cm}^{-1}$ & $-1.81059 \cdot 10^{-5}$ & 152 \\
\hline Sext. Zentrifugaldehnungskonst. $\mathrm{H}_{J K} / \mathrm{cm}^{-1}$ & $-1.7523 \cdot 10^{-6}$ & 152 \\
\hline Sext. Zentrifugaldehnungskonst. $\mathrm{H}_{J} / \mathrm{cm}^{-1}$ & $5.4320 \cdot 10^{-7}$ & 152 \\
\hline Sext. Zentrifugaldehnungskonst. $\mathrm{h}_{K} / \mathrm{cm}^{-1}$ & $3.1617 \cdot 10^{-5}$ & 152 \\
\hline Sext. Zentrifugaldehnungskonst. $\mathrm{h}_{J K} / \mathrm{cm}^{-1}$ & $-8.5420 \cdot 10^{-7}$ & 152 \\
\hline Sext. Zentrifugaldehnungskonst. $\mathrm{h}_{J} / \mathrm{cm}^{-1}$ & $2.7220 \cdot 10^{-7}$ & 152 \\
\hline
\end{tabular}

1. angeregter Zustand $\nu_{3}$

Symmetrie

Bandenposition $\tilde{\nu}_{0} / \mathrm{cm}^{-1}$

B2

Übergangsdipolmoment / D

3755.79

142

Achse des Übergangsdipolmoments

0.09714

111

Rotationskonstante $A / \mathrm{cm}^{-1}$

a

Rotationskonstante $B / \mathrm{cm}^{-1}$

26.64805

112

14.431402

112

Rotationskonstante $C / \mathrm{cm}^{-1}$

$\mathbf{9 . 1 3 8 1 6 7}$

112

Quart. Zentrifugaldehnungskonst. $\Delta_{K} / \mathrm{cm}^{-1}$

$2.8584 \cdot 10^{-2}$

112

Quart. Zentrifugaldehnungskonst. $\Delta_{J K} / \mathrm{cm}^{-1}$

$-5.6561 \cdot 10^{-3}$

112

Quart. Zentrifugaldehnungskonst. $\Delta_{J} / \mathrm{cm}^{-1}$

$1.30549 \cdot 10^{-3}$

112

Quart. Zentrifugaldehnungskonst. $\delta_{K} / \mathrm{cm}^{-1}$

$1.3261 \cdot 10^{-3}$

112 


\begin{tabular}{|c|c|c|}
\hline Größe & Wert & aus Ref. \\
\hline Quart. Zentrifugaldehnungskonst. $\delta_{J} / \mathrm{cm}^{-1}$ & $5.3817 \cdot 10^{-4}$ & 112 \\
\hline Sext. Zentrifugaldehnungskonst. $\mathrm{H}_{K} / \mathrm{cm}^{-1}$ & $8.407 \cdot 10^{-5}$ & 112 \\
\hline Sext. Zentrifugaldehnungskonst. $\mathrm{H}_{K J} / \mathrm{cm}^{-1}$ & $-1.4992 \cdot 10^{-5}$ & 112 \\
\hline Sext. Zentrifugaldehnungskonst. $\mathrm{H}_{J} / \mathrm{cm}^{-1}$ & $6.022 \cdot 10^{-7}$ & 112 \\
\hline Sext. Zentrifugaldehnungskonst. $\mathrm{h}_{K} / \mathrm{cm}^{-1}$ & $2.266 \cdot 10^{-5}$ & 112 \\
\hline Sext. Zentrifugaldehnungskonst. $\mathrm{h}_{J K} / \mathrm{cm}^{-1}$ & $1.065 \cdot 10^{-6}$ & 112 \\
\hline Sext. Zentrifugaldehnungskonst. $\mathrm{h}_{J} / \mathrm{cm}^{-1}$ & $3.087 \cdot 10^{-7}$ & 112 \\
\hline Herman-Wallis-Koeffizient $C$ & 0.0433 & 150 \\
\hline \multicolumn{3}{|c|}{ 1. angeregter Zustand $\nu_{1}$} \\
\hline Symmetrie & A1 & \\
\hline Bandenposition $\tilde{\nu}_{0} / \mathrm{cm}^{-1}$ & 3656.65 & 142 \\
\hline Übergangsdipolmoment / D & -0.02170 & 111 \\
\hline Achse des Übergangsdipolmoments & $\mathbf{b}$ & \\
\hline Rotationskonstante $A / \mathrm{cm}^{-1}$ & 27.12217 & 112 \\
\hline Rotationskonstante $B / \mathrm{cm}^{-1}$ & 14.30477 & 112 \\
\hline Rotationskonstante $C / \mathrm{cm}^{-1}$ & 9.10457 & 112 \\
\hline Quart. Zentrifugaldehnungskonst. $\Delta_{K} / \mathrm{cm}^{-1}$ & $3.0230 \cdot 10^{-2}$ & 112 \\
\hline Quart. Zentrifugaldehnungskonst. $\Delta_{J K} / \mathrm{cm}^{-1}$ & $-5.3874 \cdot 10^{-3}$ & 112 \\
\hline Quart. Zentrifugaldehnungskonst. $\Delta_{J} / \mathrm{cm}^{-1}$ & $1.2330 \cdot 10^{-3}$ & 112 \\
\hline Quart. Zentrifugaldehnungskonst. $\delta_{K} / \mathrm{cm}^{-1}$ & $1.2305 \cdot 10^{-3}$ & 112 \\
\hline Quart. Zentrifugaldehnungskonst. $\delta_{J} / \mathrm{cm}^{-1}$ & $4.8987 \cdot 10^{-4}$ & 112 \\
\hline Sext. Zentrifugaldehnungskonst. $\mathrm{H}_{K} / \mathrm{cm}^{-1}$ & $1.0016 \cdot 10^{-4}$ & 112 \\
\hline Sext. Zentrifugaldehnungskonst. $\mathrm{H}_{K J} / \mathrm{cm}^{-1}$ & $-1.418 \cdot 10^{-5}$ & 112 \\
\hline Sext. Zentrifugaldehnungskonst. $\mathrm{H}_{J} / \mathrm{cm}^{-1}$ & $4.402 \cdot 10^{-7}$ & 112 \\
\hline Sext. Zentrifugaldehnungskonst. $\mathrm{h}_{K} / \mathrm{cm}^{-1}$ & $2.166 \cdot 10^{-5}$ & 112 \\
\hline Sext. Zentrifugaldehnungskonst. $\mathrm{h}_{J} / \mathrm{cm}^{-1}$ & $2.331 \cdot 10^{-7}$ & 112 \\
\hline
\end{tabular}

Tabelle 4.4: Für die Simulation des Monomerspektrums von Wasser mit pgopher ${ }^{149}$ verwendete Parameter. Werte, die auch im Modell des starren Rotators verwendet werden, sind in fetter Schrift dargestellt.

Während das verwendete Simulationsmodell sowohl die dominierende antisymmetrische $\left(\nu_{3}\right)$ als auch die schwächere symmetrische $\left(\nu_{1}\right)$ Streckschwingung sowie in Form des Herman-Wallis-Koeffizienten näherungsweise die Kopplung zwischen der $\nu_{3}$-Schwingung und der Rotation beinhaltet ${ }^{150}$, kann die starke Coriolis-Kopplung zwischen den beiden Streckschwingungen ${ }^{111}$ aufgrund von Einschränkungen durch das Simulationsprogramm nicht berücksichtigt werden. Auch Wechselwirkungen mit dem Oberton der Knickschwingung finden in dieser Simulation keine Berücksichtigung. Zusammen mit der wahrscheinlichen Intensitätsverzerrung in den experimentellen Spektren aufgrund von Transmissionswerten nahe null für intensive Rotationsschwingungslinien können so die Diskrepanzen zwischen Messung und Modell erklärt werden. 
Die Monomer-Streckschwingungsbereiche der Spektren aus Abbildung $4.3 \mathrm{a}, \mathrm{b}, \mathrm{c}$ und e sind in Abbildung 4.6 gezeigt. Wie bereits bei den Gasphasenspektren, so sind auch hier die Spektren sowohl mit höherer Auflösung von $1-2 \mathrm{~cm}^{-1}$ als auch mit reduzierter Auflösung von $20 \mathrm{~cm}^{-1}$ zur Erfassung der allgemeinen Bandenform gezeigt. Für beide Auflösungen sind zudem simulierte Spektren unter Berücksichtigung aller in Tabelle 4.4 aufgeführten Parameter für verschiedene Temperaturen gezeigt. Insgesamt sind in den Expansionen im Gegensatz zur Gasphase sowohl die Annahmen einer Boltzmannverteilung der Zustandsbesetzung als auch der unveränderten Besetzung der Kernspinzustände ${ }^{151}$ als potentiell unzulässige Näherungen anzusehen. Zusätzlich wird in den Expansionen aufgrund des großen Durchmessers des Infrarotstrahls ein sehr heterogener Bereich untersucht, so dass gleichzeitig Moleküle innerhalb des kalten Kerns der Expansion, von warmen Aggregaten abgedampfte Monomere im Bereich der Stoßfronten und warmes Hintergrundgas sowie alle Zustände dazwischen erfasst werden und dies über einen Expansionsbereich von 0 bis etwa $2 \mathrm{~cm}$ hinter dem Düsenausgang.

Im Bewusstsein der Schwächen der Modellierung und der Heterogenität des untersuchten Bereichs soll mithilfe von Abbildung 4.6 dennoch eine Abschätzung der mittleren Monomer-Rotationstemperaturen des Wassers unter den verschiedenen experimentellen Bedingungen durch Vergleich der gemessenen mit den simulierten Spektren erfolgen. Hierbei soll mit Spektrum a) aus der klassischen, trägergasreichen Einfachschlitzdüsenexpansion mit hohem Stagnationsdruck und somit der kältesten Expansion begonnen werden. Eine Gegenüberstellung des Spektrums mit Messauflösung (links) mit dem künstlich verbreiterten (rechts) zeigt, dass sich hier aufgrund der starken PZweig-Beeinflussung durch relativ breite Bandenbeiträge der freien O-H-Streckschwingungen aggregatgebundener Moleküle die Fokussierung auf das höher aufgelöste Spektrum anbietet. Der Vergleich mit den simulierten Spektren legt hierbei aufgrund der Anzahl der in der Messung beobachteten Banden und des Intensitätsverhältnisses der drei charakteristischen starken Banden zu den schwächeren Signalen eine Rotationstemperatur um $20 \mathrm{~K}$ nahe. Das Monomerspektrum b) der Muesli-Jet-Expansion durch jede zweite der Schlitzdüsen zeigt noch immer primär die drei Banden bei 3801.5, 3779.4 und $3732.1 \mathrm{~cm}^{-1}$, nun allerdings mit einer deutlich höheren Zahl zusätzlicher schwächerer Banden, die sich zudem über einen größeren Wellenzahlbereich als in Spektrum a) verteilen. Dies lässt eine Koexistenz von zwei Temperaturen, die analog zu den Multilochdüsenexpansionen in Referenz 24 den Zonen freier Expansion und den dazwischenliegenden Kollisionszonen zugeordnet werden können, vermuten. Soll dennoch eine mittlere Temperatur bestimmt werden, so sollte für den Vergleich mit den simulierten Spektren vorwiegend der R-Zweig herangezogen werden, da dieser geringer durch Aggregatbanden beeinflusst ist. Die beste Übereinstimmung ergibt sich hierbei mit dem simulierten $50 \mathrm{~K}$-Spektrum, da hier das Intensitätsverhältnis zwischen der intensiven $3801.5 \mathrm{~cm}^{-1}$-Bande und den schwächeren Signalen bei höheren Wellenzahlen ähnlich ist. Aufgrund der deutlichen Diskrepanzen zwischen Simulation und Messung insbesondere im Bezug auf die Intensitäten der beiden anderen intensiven Banden ist hier jedoch ein Fehler von etwa $\pm 20 \mathrm{~K}$ anzunehmen. Eine Temperaturbestimmung aus 


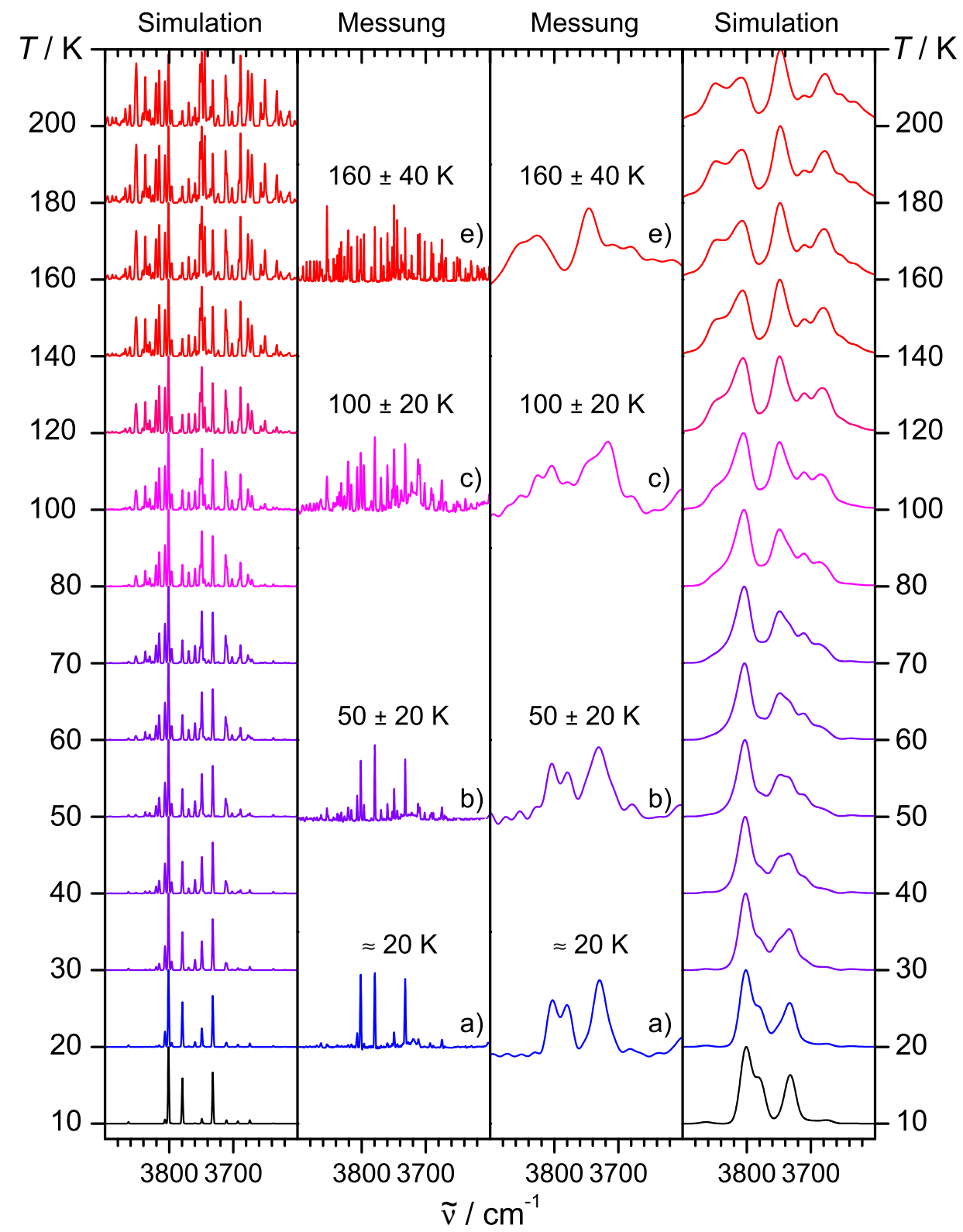

Abbildung 4.6: Abschätzung der mittleren Monomer-Rotationstemperatur bei verschiedenen Expansionsbedingungen. Vergleich der gemessenen Spektren aus Abbildung 4.3 bei Aufösungen von 1-2 $\mathrm{cm}^{-1}$ (links) und $20 \mathrm{~cm}^{-1}$ (rechts) mit pgopher-Simulationen bei entsprechenden Auflösungen und verschiedenen Temperaturen. Die Simulationsparameter wurden Tabelle 4.4 entnommen, für alle Spektren wurde eine Intensitätsnormierung vorgenommen. 
dem niedrig aufgelösten Spektrum ist aufgrund der Abweichungen von zwei wesentlich zur Gesamtintensität beitragenden Banden erneut nicht sinnvoll, so dass für Spektrum b) eine Monomer-Rotationstemperatur von $50 \pm 20 \mathrm{~K}$ angegeben werden kann. Bei der Temperaturbestimmung von Spektrum c) der wasserreichen, aber noch trägergashaltigen Expansion durch alle 20 parallelen Schlitzdüsen ist aufgrund der hohen Clusterkonzentrationen, die bereits im Übersichtsspektrum $4.3 \mathrm{c}$ deutlich wurden, erneut nur der R-Zweig für eine Temperaturabschätzung sinnvoll heranzuziehen. Aus dem relativ linearen Abfall der Intensitäten der stärkeren Banden vom Intensitätsmaximum im hochaufgelösten Spektrum (bzw. dem R-Zweig-Maximum im verbreiterten Spektrum) hin zu höheren Wellenzahlen zusammen mit der allgemeinen Breite des Streckschwingungsbereichs ergibt sich für beide Auflösungen eine maximale Übereinstimmung mit dem simulierten $100 \mathrm{~K}$-Spektrum, erneut muss jedoch ein Fehler dieser mittleren Temperaturbestimmung von $\pm 20 \mathrm{~K}$ infolge der nur partiellen Übereinstimmungen von Experiment und Simulation angenommen werden. Bei dem wärmsten Spektrum e) der trägergasfreien Multischlitzdüsenexpansion bietet sich aufgrund der Abweichungen in den Linienzahlen für höhere Temperaturen, die bereits beim Gasphasenspektrum in Abbildung 4.5 beobachtet wurden, die Zuhilfenahme der niedrig aufgelösten Spektren für die Temperaturabschätzung an. Da die Clusteranteile in dieser Expansion relativ gering sind, kann die gesamte Bandenform für die Analyse herangezogen werden. Während für den R-Zweig das Intensitätsverhältnis des Doppelmaximums die beste Übereinstimmung mit den simulierten 120 und $140 \mathrm{~K}$-Spektren zeigt, weist das der Maxima des P-Zweigs eher auf eine höhere Temperatur von etwa 180-200 K hin. Zusammen mit der zusätzlichen wahrscheinlichen Intensitätsverzerrung des experimentellen Spektrums gegenüber dem realen Verlauf durch die hohen Absorbanzen erfolgt daher eine vorsichtige Abschätzung der Monomer-Rotationstemperatur im wärmsten der Spektren auf $160 \pm 40 \mathrm{~K}$. Dieser Temperaturbereich erscheint zusätzlich durch das bekannte evaporative Limit für große Wassercluster ${ }^{153}$ von $160 \mathrm{~K}$ sinnvoll. Hierbei muss allerdings zusätzlich beachtet werden, dass insbesondere in Systemen, in denen eine Abfuhr von Kondensationswärme durch Trägergasatome nicht gegeben ist, die Temperaturen von Monomeren und Clustern nicht übereinstimmen. ${ }^{154} \mathrm{Im}$ Folgenden soll daher der Einfluss der verschiedenen Expansionsbedingungen auf die Eigenschaften und Temperaturen der erzeugten kleinen Wasseraggregate erfolgen.

\subsubsection{Einfluss der Temperatur auf Aggregatstrukturen}

Mithilfe größenselektiver Methoden wurde insbesondere für größere Wassercluster wie das Nonamer bereits ein Anstieg der strukturellen Vielfalt bei einer Temperaturerhöhung gefunden und dort mit einem Übergang zwischen festem und flüssigem Aggregatzustand verglichen. ${ }^{155}$ Für kleinere Cluster mit maximal fünf Wassermolekülen, wie sie hier betrachtet werden, sind Analogien zu Aggregatzuständen zwar nicht sinnvoll anwendbar, der Einfluss der Temperatur auf ihre Strukturen ist jedoch von mindestens ebensogroßem Interesse 


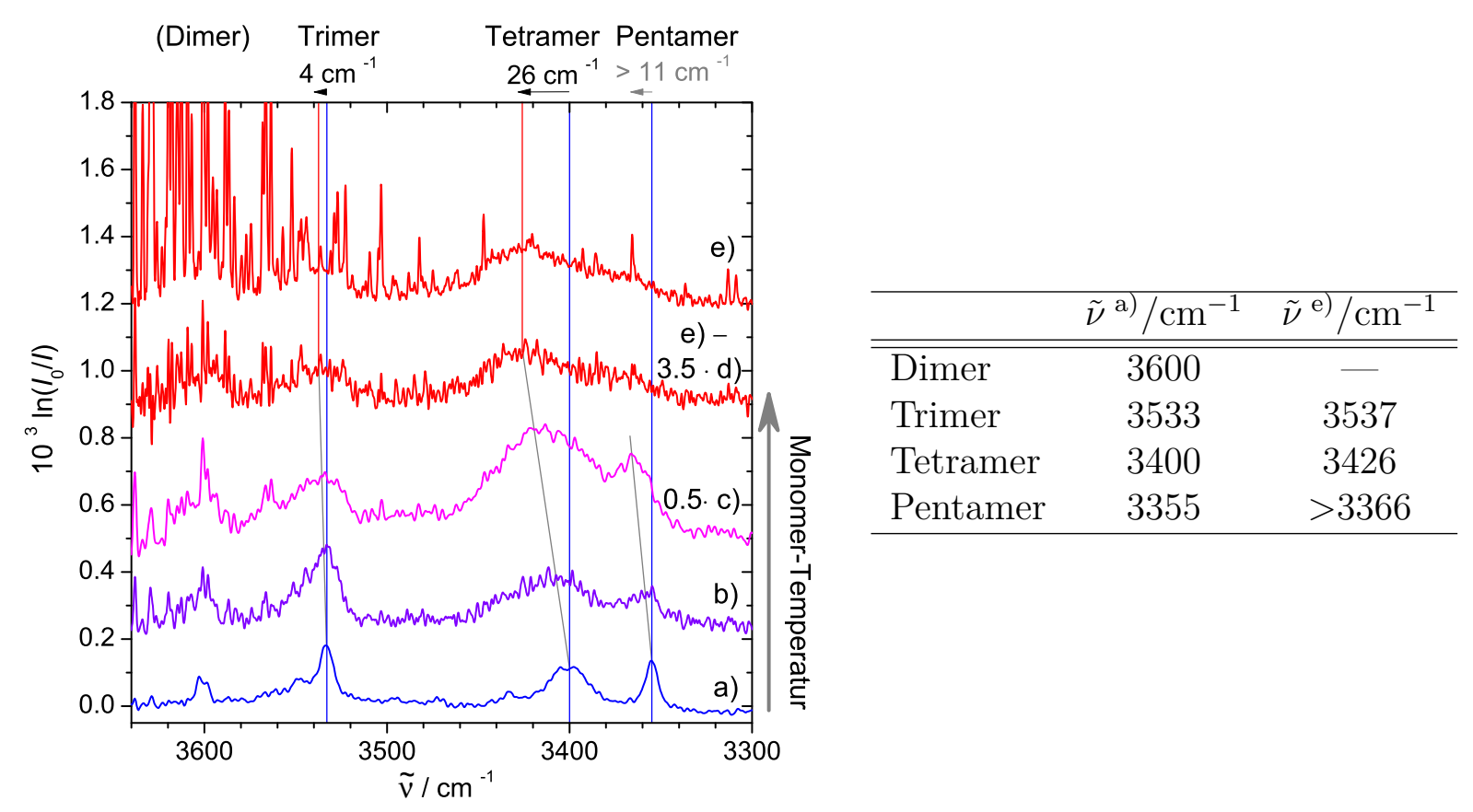

Abbildung 4.7: Einfluss der Temperatur auf die Positionen der gebundenen Cluster-O-HStreckschwingungsbanden. Die Spektren entsprechen denen aus Abbildung 4.3. Zur besseren Unterscheidung von Monomer- und Aggregatbeiträgen wird für das Spektrum bei der höchsten Monomer-Rotationstemperatur e) zusätzlich ein Differenzspektrum nach skalierter Subtraktion des reinen Monomerspektrums aus Abbildung $4.3 \mathrm{~d}$ gezeigt. Die Tabelle enthält die Peakpositionen der Clusterbanden in den Spektren der kältesten (a) und wärmsten (e) Expansionen.

Eine vergrößerte Ansicht der Übersichtsspektren aus Abbildung 4.3 im Bereich gebundener O-H-Streckschwingungen kleiner Wassercluster mit zugehörigen Wellenzahlen der Clusterbandenpeaks zeigt Abbildung 4.7. Um eine deutlichere Unterscheidung zwischen Monomer- und Aggregatbanden treffen zu können, ist für das Spektrum e) mit der höchsten Monomer-Rotationstemperatur zusätzlich ein Differenzspektrum nach skalierter Subtraktion des im Wesentlichen durch Clusterbeiträge unbeeinflussten Spektrums d) gezeigt, wodurch die Monomersignale um mindestens eine Größenordnung reduziert werden. Da mit Ausnahme von Spektrum c) Beiträge durch Wasser-Hexamere zum Spektrum weitestgehend vermieden wurden, kann im Wesentlichen jeweils eine intensive Bande pro Clustergröße vom Dimer bis zum Pentamer beobachtet werden. Die folgende Diskussion wird sich von größeren zu kleineren Aggregaten bewegen.

Die Bande des Wasser-Pentamers ist in allen in Abbildung 4.7 gezeigten Spektren zu erkennen. Ausgehend von einer Peakwellenzahl von $3355 \mathrm{~cm}^{-1}$ findet bei steigender Expansionstemperatur eine Blauverschiebung der Bande um mindestens $11 \mathrm{~cm}^{-1}$ statt. Da insbesondere für höhere Temperaturen jedoch eine deutliche Überlappung mit der Tetramerbande vorliegt und so weder Intensitäten noch Bandenpositionen verlässlich 
abgeschätzt werden können, soll an dieser Stelle keine nähere Interpretation des Bandenverhaltens erfolgen.

Die zweifach entartete, intensive Bande des Wasser-Tetramers tritt mit hoher Intensität in allen Spektren auf. Ihre Position verschiebt sich in den sukzessiv wärmer werdenden Expansionen von $3400 \mathrm{~cm}^{-1}$ unter typischen, kalten Jet-Bedingungen um insgesamt $26 \mathrm{~cm}^{-1} \mathrm{zu}$ höheren Wellenzahlen bei den milden Expansionsbedingungen von Spektrum e). Dies entspricht immerhin einer Neutralisierung von $10 \%$ der gesamten Rotverschiebung der Tetramerbande gegenüber der symmetrischen Monomer-Streckschwingung bei $3657 \mathrm{~cm}^{-1}$. Gleichzeitig findet eine deutliche Verbreiterung der Bande statt, jedoch weder eine wesentliche Änderung der Bandenform noch eine Aufspaltung der entarteten Schwingungen. Diese Reduktion der Rotverschiebung mit gleichzeitiger Bandenverbreiterung erinnert an das Verhalten des tert-Butylalkohol-Dimers in Kapitel 4.1.1. Bei einer Erwärmung können intermolekulare Schwingungsmoden wie O-O-Streckschwingungen oder OHO-Librationen angeregt werden ${ }^{33,156,157}$, die bei einer Dissoziation des Aggregats in Translations- und Rotationsfreiheitsgrade der Moleküle übergehen. Die Wasserstoffbrückenbindungen werden so geschwächt, woraus eine Blauverschiebung und inhomogene Verbreiterung der gebundenen O-H-Streckschwingungsbanden resultieren.

Für die Wasser-Trimer-Bande, die in der kältesten Expansion bei $3533 \mathrm{~cm}^{-1}$ liegt, ist die beobachtbare Blauverschiebung mit $4 \mathrm{~cm}^{-1}$ entweder als sehr klein oder aufgrund der fehlerbehafteten Bestimmung der Peakposition in der wärmsten Expansion sogar als nicht vorhanden anzusehen. Dies ist unerwartet, da im Trimer die Wasserstoffbrückenbindungen durch die hohe Ringspannung im cyclischen Aggregat, die daraus folgenden unvorteilhaften Bindungsgeometrien und geringere Kooperativität schwächer als im Tetramer sind. Wird für das Trimer ein zu den Überlegungen beim Tetramer analoges Verhalten bei der Erwärmung angenommen, also eine Aufweitung des cyclischen Aggregats durch Schwächung der Wasserstoffbrückenbindungen, ist aufgrund der geringeren Bindungsstärken ein umso größerer Effekt durch die thermische Anregung intermolekularer Moden zu erwarten. Erklärbar wird diese Diskrepanz im Verhalten von Trimer und Tetramer jedoch, wenn von einer strukturellen Veränderung des Trimers bei der Erwärmung in der Form einer Öffnung der Ringstruktur und Bildung eines flexiblen kettenförmigen Aggregats ausgegangen wird. Diese würde zwar einerseits zu einer Reduktion der Kooperativität der Wasserstoffbrückenbindungen führen, andererseits aber auch durch Aufgabe der Ringspannung eine optimalere Geometrie der einzelnen Wasserstoffbrücken ermöglichen. Gleichzeitig würde die am stärksten rotverschobene O-HStreckschwingungsbande, die im Ring nahezu keine Infrarotaktivität aufweist ${ }^{5,158}$, an Intensität gewinnen. Durch diese Kombination aus nur eingeschränkter Bindungsschwächung bei der Ringöffnung und gleichzeitiger Aktivierung der rotverschobenen Bande wird offenbar die erwartete Blauverschiebung durch Erwärmung derart kompensiert, dass die Einhüllende des Ensembles von niederfrequenten $\mathrm{O}-\mathrm{H}-$ Streckschwingungen offener, warmer Trimere an die gleiche Position wie im strukturell einheitlichen, kalten, cyclischen Aggregat rückt. 


\begin{tabular}{|c|c|c|c|c|c|c|c|c|c|}
\hline \multicolumn{2}{|l|}{$l / \mathrm{cm}$} & \multicolumn{2}{|r|}{$\begin{array}{l}\text { a) } \\
12\end{array}$} & \multicolumn{2}{|r|}{$\begin{array}{l}\text { b) } \\
11\end{array}$} & \multicolumn{2}{|r|}{$\begin{array}{l}\text { c) } \\
11\end{array}$} & \multicolumn{2}{|r|}{$\begin{array}{l}\text { e) } \\
11\end{array}$} \\
\hline & $I$ & $A$ & $c$ & $A$ & $c$ & $A$ & $c$ & $\bar{A}$ & $c$ \\
\hline Dimer & 135 & 0.66 & $2.5 \cdot 10^{12}$ & 1.58 & $6.4 \cdot 10^{12}$ & 5.89 & $2.4 \cdot 10^{13}$ & 3.95 & $1.6 \cdot 10^{13}$ \\
\hline Trimer & 450 & 1.96 & $2.2 \cdot 10^{12}$ & 4.92 & $6.0 \cdot 10^{12}$ & 7.42 & $9.0 \cdot 10^{12}$ & 3.44 & $4.2 \cdot 10^{12}$ \\
\hline Tetramer & 1120 & 3.02 & $1.4 \cdot 10^{12}$ & 6.65 & $3.2 \cdot 10^{12}$ & 29.2 & $1.4 \cdot 10^{13}$ & 7.76 & $3.8 \cdot 10^{12}$ \\
\hline
\end{tabular}

Tabelle 4.5: Abschätzung der Clusterkonzentrationen in Expansionen von Wasser bei verschiedenen Expansionsbedingungen. l: Länge des Absorptionswegs in der Expansion. $I$ : Absolute Infrarotbandenstärke gemäß Referenz $161 \mathrm{in} \mathrm{km} \mathrm{mol}^{-1}$. A: Bandenintensität in $10^{-3} \mathrm{~cm}^{-1}, A(\tilde{\nu})=\int \ln \frac{I_{0}}{I} \mathrm{~d} \tilde{\nu} . c$ : Konzentration in $\frac{1}{\mathrm{~cm}^{3}}, c=\frac{A}{I \cdot l}$.

Bei den mildesten Expansionsbedingungen und somit höchsten Temperaturen in Spektrum e) ist es eher unwahrscheinlich, dass Wasser-Dimere dort in hoher Konzentration existieren können. Mit einer (experimentell bestimmten) Dissoziationsenergie $^{159}$ von $13.2 \mathrm{~kJ} \mathrm{~mol}^{-1}$ sind sie ebenso wie Methanol-Dimere ${ }^{106}\left(13.4 \mathrm{~kJ} \mathrm{~mol}^{-1}\right)$ eher schwach gebunden. In Kapitel 4.1.1 wurden Anhaltspunkte für einen signifikanten Dissoziationsgrad von Methanol-Dimeren in deutlich kälteren Expansionen gefunden, so dass für das Wasser-Dimer ein ähnliches Verhalten zu erwarten ist. Zwar wurden auch in Wasserdampf bei $296 \mathrm{~K}$ messbare Dimeranteile nachgewiesen ${ }^{6}$, allerdings übersteigt der dort verwendete Wasserdampfdruck mit etwa 17 mbar deutlich die effektiven Wasser-Partialdrücke in den Überschallexpansionen von weniger als 1 mbar. Auch wenn in der kinetisch kontrollierten Umgebung der warmen Expansionen voraussichtlich gewisse Dimeranteile detektierbar sind, sollte dann zumindest auch ein größerer Anteil von Trimeren nachweisbar sein, da diese zum einen eine höhere Zustandsdichte aufweisen und zum anderen mit einer Energie von etwa $32 \mathrm{~kJ} \mathrm{~mol}^{-1}$ für die Dissoziation eines einzelnen Monomers aus dem Cluster ${ }^{159}$ auch deutlich stabiler sind. Dennoch ist gerade im Spektrum 4.7 e eine hohe Bandenintensität im Bereich des Wasserdimers um $3600 \mathrm{~cm}^{-1}$ zu erkennen, die daher auf Fremdbeiträge zur Gesamtintensität schließen lässt. Hierbei könnte es sich unter anderem um die zweite gebundene O-H-Streckschwingung offenkettiger Trimere handeln, eine andere Möglichkeit stellen metastabile Trimere aus einem Dimer mit einem assoziierten Monomer, wie sie in Referenz 160 beschrieben werden, dar.

Um die vermutete Bandenüberlagerung von Dimer und Trimer in den Spektren der warmen Expansionen und die Unterschiede im Strukturverhalten von Trimeren und Tetrameren weiter prüfen zu können, bietet sich eine Abschätzung der Clusterdichten über absolute und integrierte Infrarotintensitäten der Clusterbanden ${ }^{144,161}$ an. Werte für absolute Bandenstärken der gebundenen $\mathrm{O}-\mathrm{H}-\mathrm{Streckschwingungen} \mathrm{vom} \mathrm{Dimer} \mathrm{bis} \mathrm{zum}$ Tetramer wurden aus Messungen in Helium-Nanotröpfchen ${ }^{161}$ relativ zu dem bekannten Wert der asymmetrischen Monomer-Streckschwingung ${ }^{144,162}$ von $44.6 \mathrm{~km} \mathrm{~mol}^{-1}$ erhalten. Während durch die andere Umgebung in Heliumtröpfchen gegenüber den hier verwendeten Gasphasenexpansionen kein Effekt bei den absoluten Intensitäten der 


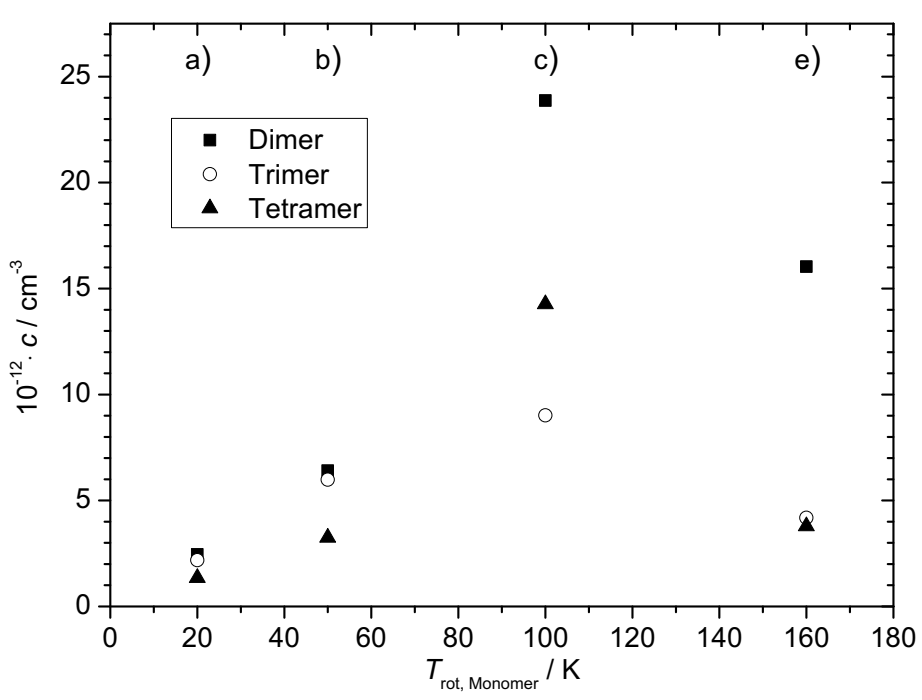

Abbildung 4.8: Veranschaulichung der Clusterkonzentrationen aus Tabelle 4.5 in Abhängigkeit von der abgeschätzten Monomer-Rotationstemperatur aus Abbildung 4.6. Die Kennbuchstaben beziehen sich auf die Spektrenbenennung in Abbildung 4.3.

Clusterbanden $\mathrm{zu}$ erwarten ist ${ }^{163}$, ist von Beeinflussungen durch die bei steigender Temperatur sinkenden Infrarotintensitäten wasserstoffbrückengebundener O-H-Streckschwingungen und somit von zu kleinen Werten für berechnete Konzentrationen auszugehen. ${ }^{102}$ Da die Verstärkung der Clusterbanden gegenüber dem Monomer für die verschiedenen betrachteten Aggregate eine Größenordnung umspannt, werden Temperatureffekte hier nicht zu vernachlässigen sein, ebenso wie der Einfluss einer Ringöffnung cyclischer Cluster auf die Intensitäten.

Die Konzentration $c$ eines Clusters kann aus der integrierten natürlichen Absorbanz $A$ der zugehörigen Bande, der absoluten Infrarotbandenstärke $I$ und dem Absorptionsweg $l$ gemäß

$$
c=\frac{A}{I \cdot l}
$$

berechnet werden. Dabei ist zu bedenken, dass der Absorptionsweg für die verwendete Einfachschlitzdüse mit $l^{\text {a }}=12 \mathrm{~cm}$ nicht mit dem Wert für die Mehrfachschlitzdüse übereinstimmt, der aufgrund des Ausbreitungswinkels der Expansionen auf $l^{\mathrm{b}, \mathrm{c}, \mathrm{e}} \approx$ $11 \mathrm{~cm}$ abgeschätzt wird.

Tabelle 4.5 enthält die Literaturwerte der absoluten Clusterbandenstärken für Wasserdimere, -trimere und -tetramere sowie die aus den Spektren 4.7 a, b, c und e bestimmten Bandenintegrale sowie die gemäß Gleichung 4.4 berechneten Clusterkonzentrationen in $\frac{1}{\mathrm{~cm}^{3}}$. Zur besseren Veranschaulichung der Entwicklungen sind zusätzlich in Abbildung 4.8 die Konzentrationen für die einzelnen Clustergrößen als Funktion der den jeweiligen Spektren zugehörigen abgeschätzten Monomer-Rotationstemperaturen aus Abschnitt 4.2.1 aufgetragen. Bei der Auswertung muss beachtet werden, dass 
bei der Konzentrationsberechnung keine Anpassungen der absoluten Intensitäten auf strukturelle Änderungen innerhalb der Cluster, Bandenüberlagerungen oder erhöhte Temperaturen vorgenommen wurden.

Für die kalte Einfachschlitzdüsenexpansion a) zeigt Abbildung 4.8 ein Absinken der Clusterkonzentration mit steigender Aggregatgröße, dabei bewegen sich die Werte der Absolutkonzentrationen im Prozentbereich der geschätzten Monomerkonzentration. Mit etwa $10^{12}$ Molekülen pro Kubikzentimeter stimmen die Cluster-Konzentrationen dabei in etwa mit den durch Paul et al. in Helium/Wasser-Schlitzdüsenexpansionen ${ }^{133}$ gefundenen überein, wobei dort zwar eine geringere Schlitzbreite ${ }^{164}$ von nur $0.127 \mathrm{~mm}$, dafür aber höhere Wasseranteile im Expansionsgasgemisch eingesetzt wurden. Für die noch relativ kalte 10-Düsen-Expansion b) findet etwa eine Verdopplung der Konzentrationen statt, die die gegenüber a) etwa zweimal so große Wasserkonzentration im Expansionsgasgemisch widerspiegelt. Die Konzentrationsverhältnisse zwischen den verschiedenen Clustern bleiben nahezu unverändert.

Eine deutliche Veränderung der relativen Aggregatkonzentrationen findet jedoch in Spektrum 4.7 c statt. Die insgesamt höheren Bandenintensitäten können hier zwar sowohl auf real größere Konzentrationen durch den höheren Wasseranteil im Expansionsgasgemisch als auch auf Überlappung mit Hexamerbanden ${ }^{165}$ zurückgeführt werden. Dies erklärt jedoch nicht das unerwartete Dimer:Trimer:Tetramer Konzentrationsverhältnis von etwa 5:2:3 mit sowohl einer ungewöhnlich hohen Dimerkonzentration als auch einer sehr geringen Trimerkonzentration, die formal sogar geringer als die des Tetramers ist. Ein derartiges Verhalten wird üblicherweise bei kooperativen Wasserstoffbrückenbildnern und einer Erhöhung der mittleren Clustergröße nicht beobachtet. ${ }^{133}$ Es ist jedoch konsistent mit der Hypothese eines signifikanten Anteils kettenförmiger Wasser-Trimere in der Expansion, die sowohl eine gegenüber dem ringförmigen Trimer geringere absolute Bandenstärke als auch zusätzliche Intensität im Bereich der Dimerbande aufweisen. Diese offenen Aggregate müssten zusätzlich noch eine weitere infrarotaktive Bande der freien $\mathrm{O}-\mathrm{H}$-Streckschwingungen der terminalen Wassermoleküle besitzen, die sich leicht von der Dimer-Akzeptorbande ${ }^{166}$ bei $3730 \mathrm{~cm}^{-1}$ unterscheiden sollte, unter dem breiten Monomerbereich in Abbildung $4.5 \mathrm{c}$ jedoch nicht eindeutig erkennbar ist.

In der wärmsten Expansion (Spektrum e) ist aufgrund der fehlenden Wärmeabfuhr durch ein Trägergas die mittlere Clustergröße wieder geringer als in Spektrum c. Dennoch ist die aus den Bandenintensitäten berechnete Häufigkeit des Trimers nur etwa so hoch wie die des Tetramers, während die Dimerbande erneut eine sehr hohe Intensität aufweist. Das Integral ist aufgrund der starken Überlappung mit den Monomerlinien fehlerbehaftet, doch es ergibt sich näherungsweise ein Konzentrationsverhältnis Dimer:Trimer:Tetramer von 4:1:1. Auch hier sind also wieder Anzeichen für offenkettige Wasser-Trimere mit einem Intensitätstransfer vom Trimer- in den Dimerbereich zu finden. Aufgrund dieser wahrscheinlichen Bandenüberlappung im Spektralbereich um $3600 \mathrm{~cm}^{-1}$ kann keine separate Untersuchung des Wasser-Dimers bei einer graduellen Erwärmung der Expansionen erfolgen. Es liegt jedoch die Vermutung nahe, dass in 
den warmen Expansionen sowohl gebundene als auch metastabile Dimere sowie durch Kollisionspaare induzierte Absorption im Dimerbereich vorliegen. ${ }^{135,160}$

Anhand der rein experimentell basierten Auswertung können daher folgende begründete Vermutungen über die Strukturen von kleinen Wasserclustern in Überschallexpansionen angestellt werden: Während in kalten Expansionen Aggregate aus drei bis fünf Molekülen als stabile Cyclen vorliegen ${ }^{3}$, findet in warmen Expansionen eine Aufweitung der Ringstruktur des Tetramers (und Pentamers) durch thermische Anregung von intermolekularen Schwingungsmoden statt. Im Falle des Trimers hingegen wird zumindest für einen signifikanten Anteil der Cluster eine Bildung offener, flexibler Strukturen erfolgen. Aussagen über das Dimer können aufgrund von Bandenüberlagerungen nicht getroffen werden.

Offene Wassercluster sind kein vollständig neues Phänomen, sie werden als Bestandteil von Wasser im überkritischen Zustand angenommen ${ }^{167,168}$ und können durch Koaggregation mit Kationen wie Alkalimetallionen ${ }^{169}$ oder Alkylammoniumionen ${ }^{170}$ auch in Expansionen bzw. in der Gasphase stabilisiert werden. Die Unterschiede im Verhalten der verschiedenen Clustergrößen erinnern zudem an die Strukturen von Mischclustern eines Benzolmoleküls mit Methanol, da auch hier eine offenkettige Struktur für das Methanol-Trimer und die cyclische Form für das Tetramer beobachtet wurden. ${ }^{171}$ Analoge Experimente mit Wasser zeigten jedoch eine Ringöffnung erst ab sechs $\mathrm{H}_{2} \mathrm{O}$-Molekülen. ${ }^{172}$

Zur Überprüfung der Spektreninterpretationen ist eine Unterstützung durch theoretische Vorhersagen wünschenswert. Während die Geometrieoptimierungen für kleine, cyclische Wassercluster unkompliziert sind, existiert für offenkettige, kleine Aggregate typischerweise keine ausreichende energetische Barriere, um die Umwandlung in die cyclische Form zu unterbinden und lokale offene Minimumstrukturen zu erhalten. Zwar konnten derartige Strukturen mithilfe einzelner, eher weniger anspruchsvoller Rechnungen erhalten werden ${ }^{173,174}$, hierbei handelt es sich jedoch vermutlich um methodenspezifische Artefakte. Alternativ wurden offenkettige Strukturen mittels molekulardynamischer Rechnungen unter überkritischen Bedingungen erhalten ${ }^{175,176}$, diese sind jedoch nicht uneingeschränkt übertragbar und enthalten keine Berechnungen der Normalschwingungen der Aggregate. Weiterhin wurden Optimierungen kettenförmiger, kleiner Wasseraggregate unter Simulation eines externen elektrischen Felds durchgeführt. ${ }^{177}$ Um jedoch Vorhersagen über die O-H-Streckschwingungen offener WasserTrimere und -Tetramere in Überschallexpansionen treffen zu können, ist die Einführung zusätzlicher struktureller Randbedingungen, die eine Cyclisierung der Aggregate verhindert, notwendig. Obwohl diese Arbeit in ihrem Schwerpunkt experimentell angelegt ist, sollen hier zwei Ansätze der Modellierung kettenförmiger Strukturen verfolgt werden.

Abbildung 4.9 zeigt den Vergleich der Clusterbereiche des jeweils kältesten (a) und wärmsten (e-3.5·d) Spektrums aus Abbildung 4.7 mit zwei verschiedenen theoretischen Modellen zur Vorhersage des Schwingungsspektrums cyclischer und offenkettiger Wasser-Trimere und -Tetramere. Im ersten Fall (mittlerer Kasten) wird eine kettenartige 


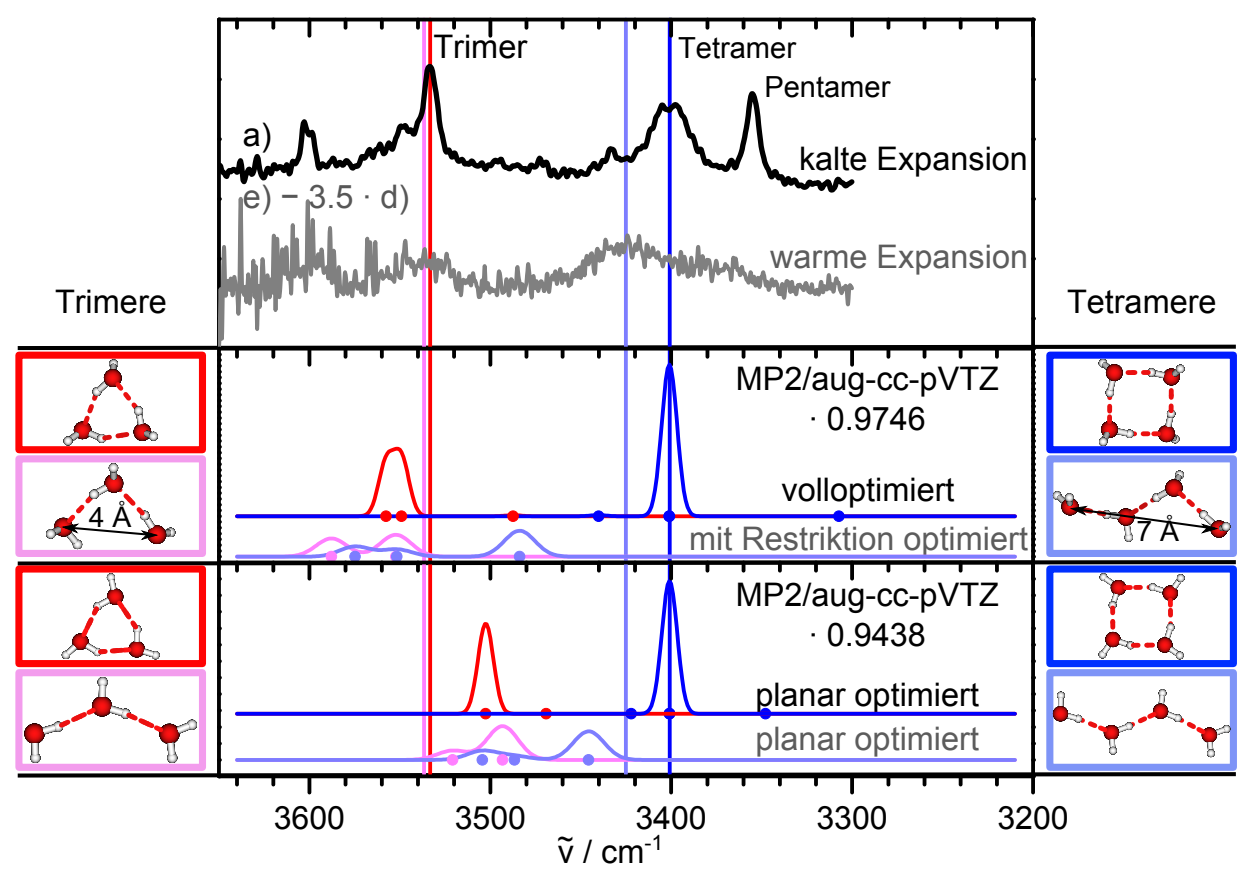

Abbildung 4.9: Vergleich warmer (Abbildung 4.3 (e-3.5·d) und kalter (Abbildung 4.3 a) experimenteller Wasserspektren mit berechneten ${ }^{117}$ Wellenzahlen für cyclische und offenkettige Trimer- und Tetramerstrukturen. Mittlerer Kasten: Volle Optimierung für Ringstrukturen, fixierter O-O-Abstand für Kettenstrukturen. Unterer Kasten: Alle Strukturen wurden unter der Voraussetzung vollständiger Planarität optimiert. Wellenzahlen wurden auf MP2/aug-cc-pVTZ-Niveau optimiert und zwecks Übereinstimmung der dominanten Bande des cyclischen Tetramers skaliert. Punkte auf Basislinienniveau geben die Position von $\mathrm{O}-\mathrm{H}-\mathrm{Streckschwingungsbanden} \mathrm{unabhängig} \mathrm{von}$ ihrer Intensität an. Simulierte Bandenstärken wurden für Trimere verdoppelt um ihre größere Anzahl in der Expansion wiederzugeben. Die Bandenformen wurden durch Gaußverteilungen mit einer vollen Halbwertsbreite von $10 \mathrm{~cm}^{-1}$ (Ringe) bzw. $20 \mathrm{~cm}^{-1}$ (Ketten) erhalten.

Struktur der Aggregate dadurch erhalten, dass ein Sauerstoff-Sauerstoff-Abstand auf einen so hohen Wert fixiert wird (Trimer: $4 \AA$, Tetramer: $7 \AA$ ), dass eine Cyclisierung des Aggregats unterbunden wird. Hierdurch findet zwar eine Analyse der Normalmoden an einem nicht-stationären Punkt der Potentialhyperfläche statt, der Effekt auf die hochfrequenten $\mathrm{O}-\mathrm{H}-\mathrm{Streckschwingungen} \mathrm{sollte} \mathrm{jedoch} \mathrm{gering} \mathrm{sein,} \mathrm{so} \mathrm{dass} \mathrm{die} \mathrm{erhaltenen}$ Spektrenvorhersagen noch einen qualitativen Aussagegehalt besitzen. Zum Vergleich wurden zusätzlich die Spektren der volloptimierten Ringstrukturen berechnet. In einem zweiten Ansatz (unterer Kasten in Abbildung 4.9) wurden Ketten- und Ringaggregate ausgehend von vollständig planaren Strukturen optimiert, wodurch eine ausreichend hohe energetische Barriere generiert wird, um eine Relaxation in die globalen Minimumstrukturen zu verhindern. Auf diese Weise werden die Cluster an einem Sattelpunkt 
höherer Ordnung untersucht, woraus kleine, imaginäre Wellenzahlen für die intermolekularen Schwingungen resultieren. Erneut sollten jedoch die hochfrequenten Moden im Vergleich zu einem realen Trimer mit gleicher Sauerstoffatomanordnung nur geringfügig beeinflusst sein. Für beide Herangehensweisen wurden Rechnungen auf MP2/aug-ccpVTZ-Niveau durchgeführt und die erhaltenen harmonischen Schwingungswellenzahlen auf eine Übereinstimmung der intensiven, zweifach entarteten Streckschwingung des cyclischen Wasser-Tetramers mit der experimentellen Bandenposition skaliert. Trimerintensitäten wurden mit dem Faktor 2 multipliziert, um ihre höhere Konzentration in den Expansionen nachzuahmen. Die theoretischen Linienspektren sind in Abbildung 4.9 als Gaußprofile mit einer vollen Halbwertsbreite von $10 \mathrm{~cm}^{-1}$ für ringförmige Aggregate und $20 \mathrm{~cm}^{-1}$ für Ketten dargestellt. Blaue Banden gehören zu Tetramerstrukturen, rote zu Trimeren, blasse Farben kennzeichnen offenkettige Strukturen. Punkte auf der Basislinie geben die Positionen von Schwingungsbanden unabhängig von ihrer Infrarotaktivität an. Diese Modellierungsansätze ersetzen keine volldimensionalen, anharmonischen Berechnungen, können aber qualitative Hinweise im Bezug auf die Strukturen von Wasser-Trimeren und -Tetrameren in warmen Überschallexpansionen liefern.

Nach der Skalierung zeigt sich, dass die rechnerische Wellenzahl der intensivsten Bande für das volloptimierte Trimer etwas zu hoch und für das planare cyclische Trimer etwas zu niedrig ist. Beide Methoden sagen voraus, dass bei einer Ringöffnung die am weitesten rotverschobene, zuvor praktisch infrarotinaktive Bande im offenkettigen Trimer die intensivste darstellt und jeweils etwa an der Position der im kalten Ringtrimer stärksten Bande liegt. Sie entsteht durch die phasengleiche Streckschwingung der beiden wasserstoffbrückengebundenen $\mathrm{O}-\mathrm{H}$-Bindungen und weist die größte Amplitude am reinen Donor-Wassermolekül auf. Die gegenphasige Streckschwingung, die stärker am mittleren Donor-Akzeptor-Wassermolekül lokalisiert ist, weist eine höhere Wellenzahl auf und könnte zumindest im Modell des fixierten O-O-Abstands zur Intensität im Dimerbereich beitragen. Die Dissoziationsenergien der offenkettigen Aggregate liegen etwa $15-20 \mathrm{~kJ} \mathrm{~mol}^{-1}$ unterhalb derjenigen der cyclischen Cluster.

Für das Tetramer sagen beide Methoden ebenfalls die IR-Aktivierung der am weitesten rotverschobenen Bande bei einer Ringöffnung voraus, sie wird zur stärksten Bande des Spektrums und wird im Wesentlichen durch eine lokalisierte Streckschwingung des gebundenen $\mathrm{O}-\mathrm{Hs}$ des von der Donorseite aus zweiten Wassermoleküls charakterisiert. Die Blauverschiebung gegenüber der Position im cyclischen Aggregat ist so stark, dass die am stärksten rotverschobene Bande des offenen Tetramers selbst eine höhere Wellenzahl aufweist als die im cyclischen Tetramer entartete, intensive Bande bei $3400 \mathrm{~cm}^{-1}$, die bei der Ringöffnung, wie es durch die Symmetrieerniedrigung der Clusters zu erwarten ist, energetisch aufspaltet. Die Wellenzahländerung der am weitesten rotverschobenen Bande bei der Öffnung um mehr als $100 \mathrm{~cm}^{-1}$ kann bereits als Hinweis auf eine enorme Schwächung der Wasserstoffbrückenbindungen gesehen werden, die aufgrund der fehlenden Ringspannung deutlich größer als beim Trimer ausfällt. Dies wird durch eine Energiedifferenz von etwa $40 \mathrm{~kJ} \mathrm{~mol}^{-1}$ zum cyclischen Tetramer unterstützt. 
a)
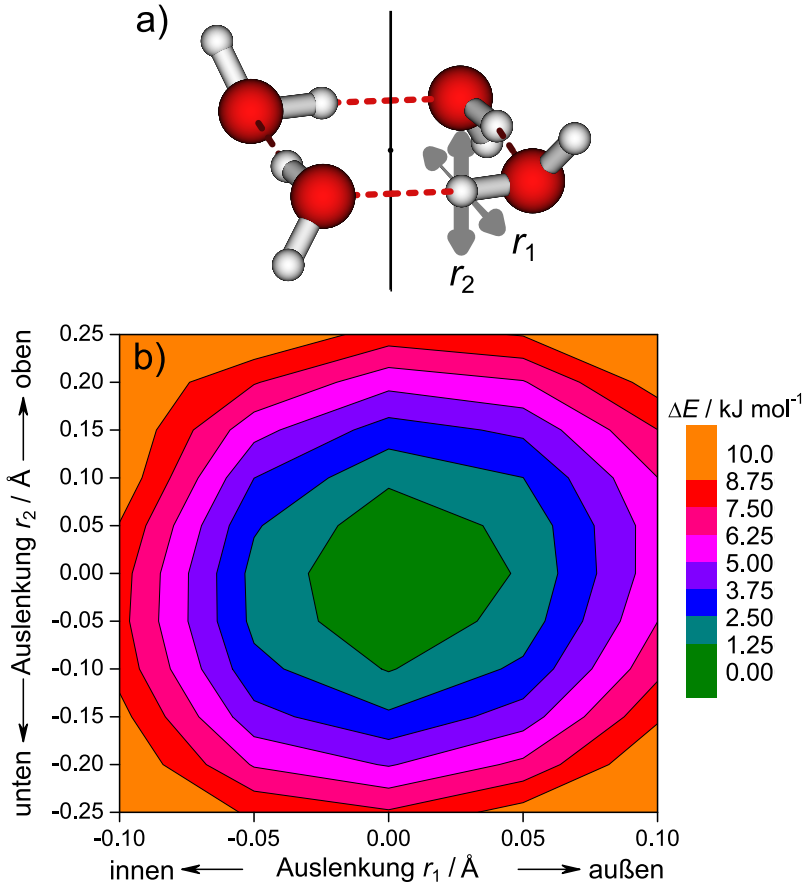

c)

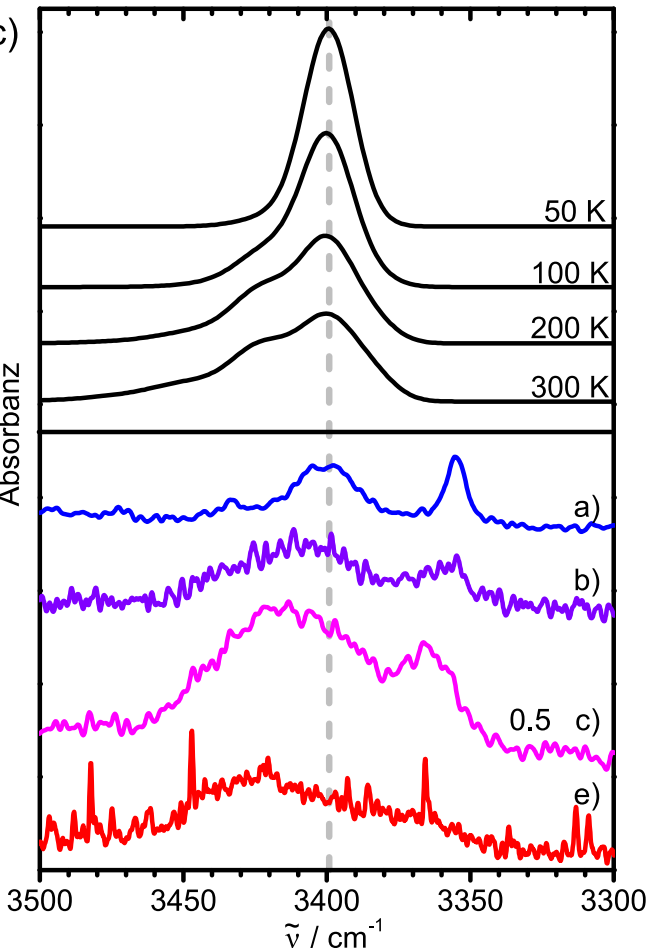

Abbildung 4.10: Simulation der Auswirkung der Anregung von Librationsschwingungen des Wasser-Tetramers auf die $\mathrm{O}-\mathrm{H}$-Streckschwingungen. a) Molekülgeometrie unter Erhalt der $\mathrm{S}_{4}$-Symmetrie mit exemplarischer Auslenkung eines wasserstoffbrückengebundenen Wasserstoffatoms. b) Energiedifferenzen zwischen Strukturen mit ausgelenkten H-Atomen und der Gleichgewichtsstruktur. c) MP2/aug-cc-pVTZ-Simulation der Auswirkungen von Librationsanregungen auf die O-H-Streckschwingungen. Oben: Boltzmann-gemittelte theoretische Schwingungsspektren aus Gaußprofilen ( $\mathrm{FWHM}=20 \mathrm{~cm}^{-1}$ ) bei verschiedenen Temperaturen, Wellenzahlen wurden mit dem Faktor 0.974 skaliert. Unten: Spektrenausschnitte der Tetramer- (und Pentamer-)Banden aus Abbildung 4.3.

Die infolge einer Ringöffnung vorhergesagte Blauverschiebung der intensivsten Tetramerbande im Spektrum entspricht zwar dem beobachteten Verhalten im Experiment bei einem Vergleich von kalten mit warmen Expansionen, gleichzeitig ist dies jedoch auch der zu erwartende Effekt bei einer thermischen Anregung niedriger intermolekularer Schwingungen, insbesondere der Librationen. ${ }^{156}$ Um auch den Einfluss der Anregung von Librationsschwingungen auf die $\mathrm{O}-\mathrm{H}-\mathrm{Streckschwingungsbanden} \mathrm{des} \mathrm{Tetramers} \mathrm{ab-}$ schätzen zu können, wurde der folgende Ansatz gewählt.

Ausgehend von der $\mathrm{S}_{4}$-symmetrischen Minimumstruktur des cyclischen Wasser-Tetramers auf MP2/aug-cc-pVTZ-Niveau wird der Abstand der Sauerstoffatome zueinander aufgrund der Annahme adiabatischer Separierung der niederfrequenten O-OStreckschwingungen von den Librationsschwingungen konstant gehalten. Die Koordi- 
naten der vier wasserstoffbrückengebundenen Wasserstoffatome werden in den in Abbildung 4.10 a exemplarisch gezeigten Richtungen auf einem Raster mit $0.05 \AA$ großen Abständen variiert: Einmal parallel zur Symmetrieachse und einmal sowohl senkrecht zur Symmetrie- als auch zur zugehörigen O-O-Achse. In der dritten Raumrichtung sowie für alle Koordinaten der freien Wasserstoffatome wird eine Optimierung zugelassen, jedoch immer unter Erhalt der $\mathrm{S}_{4}$-Symmetrie. Dieses Vorgehen entspricht keiner vollen, achtdimensionalen Modellierung der Librationsmoden und vernachlässigt zudem alle Geometrien, die zu einem Symmetriebruch führen würden und ergibt somit zwingend zu hohe Werte bei Temperaturabschätzungen, sollte für qualitative Aussagen jedoch geeignet sein.

Für alle 46 Punkte auf dem Raster, die zu Energien von höchstens $10 \mathrm{~kJ} \mathrm{~mol}^{-1}$ über der der volloptimierten Struktur führen, wurden Strukturoptimierungen und harmonische Frequenzrechnungen durchgeführt, Werte der unskalierten Streckschwingungswellenzahlen, Bandenstärken und Energien können dem Anhang A.4 entnommen werden. Abbildung $4.10 \mathrm{~b}$ zeigt die (nicht nullpunktskorrigierten) Energiedifferenzen zwischen den 46 berechneten Strukturen mit verschiedenen Auslenkungen der Wasserstoffatome und der volloptimierten Struktur. Es zeigt sich, dass eine Auslenkung der wasserstoffbrückengebundenen H-Atome parallel zur Symmetrieachse, entsprechend einer Librationsschwingung bei etwa $400 \mathrm{~cm}^{-1}$, zu deutlich geringeren Energieanstiegen führt als eine Auslenkung orthogonal dazu, entsprechend einer Libration mit typischer Wellenzahl von $800 \mathrm{~cm}^{-1}$. Somit wird auch die thermische Anregung der Moden verstärkt parallel zur Symmetrieachse erfolgen.

Eine Modellierung der Auswirkungen von Librationsanregungen auf die zweifach entartete O-H-Streckschwingungsbande zeigt Abbildung 4.10 c. Hierzu wurden alle Wellenzahlen mit dem Faktor 0.974 skaliert um eine Übereinstimmung der experimentellen Bande in kalten Expansionen mit der entsprechenden Schwingung der Gleichgewichtsstruktur zu erhalten. Die Schwingungsintensitäten der einbezogenen 46 Geometrien wurden für die gezeigten Temperaturen von 50, 100, 200 und $300 \mathrm{~K}$ boltzmanngewichtet skaliert und die Strichspektren mit Gaußprofilen der vollen Halbwertsbreite von $20 \mathrm{~cm}^{-1}$ gefaltet. Abbildung 4.10 c zeigt die resultierenden simulierten Banden im Vergleich zu den Tetramerbanden der Spektren aus Abbildung 4.7. Banden in den experimentellen Spektren unterhalb von $3400 \mathrm{~cm}^{-1}$ sind auf Pentamerbeiträge zurückzuführen, scharfe Peaks in Spektrum e) werden durch den Oberton der Monomer-Knickschwingung verursacht. Die Bandenmodellierung zeigt eine qualitativ gute Wiedergabe des experimentellen Verhaltens der Tetramerbande mit einer Verbreiterung und gleichzeitigen Blauverschiebung bei Erwärmung. Abweichungen in der Intensitätsverteilung können auf Überlagerungen mit anderen Clustergrößen oder -strukturen zurückgeführt werden. Insgesamt kann das Verhalten der Tetramerbande mit steigenden Expansionstemperaturen gut mit einer sukzessiven Erhöhung der Clustertemperaturen von 50 auf bis zu $300 \mathrm{~K}$ entsprechend einer nahezu nichtexistenten Kühlung der betreffenden Schwingungsfreiheitsgrade in der Expansion beschrieben werden. Auch unter Einbeziehung der modellinhärenten Temperaturüberschätzung kann daher gefolgert werden, dass die 
Schwingungstemperaturen deutlich oberhalb der ermittelten Monomer-Rotationstemperaturen von 20 bis $160 \mathrm{~K}$ liegen, dies bestätigt erneut die effizientere Kühlung von Rotations- gegenüber Schwingungsfreiheitsgraden und wird sicherlich auch durch die im Spektrenverlauf von a) nach e) abnehmende Möglichkeit der Kondensationswärmeabfuhr durch Trägergasstöße befördert.

Insgesamt unterstützen diese theoretischen Ansätze und Modellierungen die Deutung einer Bildung offenkettiger Wasser-Trimere in warmen Expansionen, während für Tetramere die Effekte durch Ringöffnung und -aufweitung auf das Spektrum gegenüber kälteren Bedingungen nicht zweifelsfrei zu unterscheiden sind. Die fehlende Ringspannung und die große rechnerische Energiedifferenz zwischen cyclischem und offenkettigem Tetramer von $40 \mathrm{~kJ} \mathrm{~mol}^{-1}$ legt jedoch die bevorzugte Bildung ringförmiger Tetramere auch unter wärmeren Bedingungen nahe.

\subsection{Bildung von Mischaggregaten - Wasser und Deuteriumoxid}

Die Spektren der synchronen Expansion von Methanol und tert-Butylalkohol in Kapitel 4.1 haben gezeigt, dass mit der konvergierenden Schlitzdüsenanordnung eine Bildung gemischter Cluster in der Expansion erfolgt. Die Ergebnisse haben zudem nahegelegt, dass zumindest innerhalb der typischen Messabstände zur Düse von 1 bis $3 \mathrm{~cm}$ Isotopenaustauschprozesse zwischen den Komponenten unterdrückt werden können, wobei dies nur für Monomere und Dimere in der Expansion zweifelsfrei festgestellt werden konnte. Um auch einen Austausch in größeren, cyclischen Aggregaten ausschließen zu können, bietet sich das System $\mathrm{H}_{2} \mathrm{O} / \mathrm{D}_{2} \mathrm{O}$ an. Ein Wasserstoffaustausch innerhalb von Ringaggregaten, der den wahrscheinlichen Mechanismus für einen Isotopenaustausch darstellt, führt hier zur Bildung von Hydrodeuteriumoxid (HDO), dessen Knickschwingung eine von denen des $\mathrm{H}_{2} \mathrm{O}$ und $\mathrm{D}_{2} \mathrm{O}$ um jeweils etwa $200 \mathrm{~cm}^{-1}$ separierte Wellenzahl aufweist ${ }^{142}$ und somit im Spektrum leicht identifiziert werden kann. Diese Separation existiert dabei gleichermaßen für Monomere wie für Cluster, da das Ausmaß der Aggregation nur einen geringen Einfluss auf die Lage der Deformationsschwingungen besitzt. ${ }^{178,179,180,181}$ Für den Umlagerungsmechanismus legen die Höhen der Energiebarrieren für cyclische Fluorwasserstoffaggregate ${ }^{182}$ nahe, dass für den Wasserstoff-/Deuteriumaustausch in größeren Ringclustern eine Konkurrenz zwischen verschiedenen konzertierten und sequentiellen Mechanismen besteht.

Sollte sich auch bei den Wasserisotopologen kein Hinweis auf einen Isotopenaustausch innerhalb der Expansion finden, dann bietet die synchrone Expansion von $\mathrm{H}_{2} \mathrm{O}$ und $\mathrm{D}_{2} \mathrm{O}$ gegenüber der vorgemischten deutliche Vorteile für die Untersuchung der Spektren gemischter Aggregate, da ohne die Bildung von HDO die Anzahl an auftretenden Isotopomeren und Isotopologen für jede Aggregatgröße deutlich sinkt. Für das Dimer ergäbe sich beispielsweise bei Unterdrückung des Donoraustauschs eine Reduk- 
tion von 12 auf 4 mögliche Isotopenkombinationen, für das cyclische Trimer bereits von 24 auf 4 und für das Tetramer von 70 auf 6. Während bei den Wasser-Dimeren die intensive Streckschwingung der wasserstoffbrückengebundenen Hydroxylgruppe im Wesentlichen abhängig vom Wasserstoffisotop der Donorgruppe ist und insbesondere die Wellenzahländerung bei einem Austausch des Akzeptors unterhalb von $1 \mathrm{~cm}^{-1} \operatorname{liegt}^{179}$, ist eine Identifizierung einzelner Spezies für Trimere vielversprechender. Während bei vorgemischten Komponenten die Anteile von $\left(\mathrm{H}_{2} \mathrm{O}\right)_{2}\left(\mathrm{D}_{2} \mathrm{O}\right)_{1}$ und $\left(\mathrm{H}_{2} \mathrm{O}\right)_{1}\left(\mathrm{D}_{2} \mathrm{O}\right)_{2}$ jeweils nie mehr als $7 \%$ der Gesamttrimermenge ausmachen ${ }^{183}$, stellen sie bei einer austauschfreien Synchronexpansion die einzigen gemischten Trimerspezies dar. Gerade im $\mathrm{O}-\mathrm{H}-$ Streckschwingungsbereich, wo anders als im Ferninfraroten ${ }^{184}$ keine Rotationsauflösung der einzelnen Spezies möglich sein dürfte, ist eine solche Reduktion auf wenige Komponenten besonders von Vorteil.

Bei der experimentellen Umsetzung wurde wie folgt vorgegangen. Die Wasserkonzentrationen in reinen $\mathrm{H}_{2} \mathrm{O}$ - und $\mathrm{D}_{2} \mathrm{O}$-Expansionen durch alle 20 Schlitze des Muesli-JetAufbaus mit konvergierender Düsenanordnung wurden so eingestellt, dass bei einem Stagnationsdruck von 1000 mbar für die Aggregatgrößen vom Dimer bis zum Pentamer gut sichtbare, separierte Banden vorlagen. Im Anschluss wurden bei gleichbleibenden Bedingungen Spektren der synchronen Expansion beider Komponenten durch jeweils die Hälfte der Schlitzdüsen sowie der Expansion der vorgemischten Komponenten durch alle Düsen gemessen. Bei der typischen Substanzaufnahme, bei der die Probensubstanz im Sättiger vom Trägergas durchströmt wird, wären für die angestrebten Konzentrationen Sättigertemperaturen unterhalb von $0^{\circ} \mathrm{C}$ notwendig gewesen. Das Ausfrieren des Wassers im Sättiger hätte jedoch zu schwankenden Konzentrationen im Expansionsgemisch geführt und auch ein Einfüllen als Feststoff analog zum tert-Butylalkohol (siehe Abschnitt 4.1) erwies sich aufgrund des niedrigeren Schmelzpunkts von Wasser und des potentiellen Isotopenaustauschs von $\mathrm{D}_{2} \mathrm{O}$ mit der Luftfeuchtigkeit als nicht praktikabel. Der apparative Aufbau lässt aktuell eine gleichzeitige Helium-Zumischung für beide Reservoire nicht zu, zudem hat sich die Konstanz dieser Verdünnungsmethode über mehrere Messtage als mäßig erwiesen. Daher wurde eine Substanzzufuhr gewählt, bei der mittels einer Verringerung der Kontaktfläche zwischen dem Trägergas Helium und dem (flüssigen) Wasser die Konzentration verringert wurde. Hierzu wurden je 9 Glasgefäße mit einem Volumen von ungefähr $2 \mathrm{~mL}$ und einer Öffnungsfläche von etwa $0.2 \mathrm{~cm}^{2}$ befüllt und in die Sättiger eingebracht. Gleichzeitig wurden die Sättigertemperaturen auf $40{ }^{\circ} \mathrm{C}\left(\mathrm{H}_{2} \mathrm{O}\right)$ und $50{ }^{\circ} \mathrm{C}\left(\mathrm{D}_{2} \mathrm{O}\right)$ erhöht. So wurde zwar kein Konzentrationsverhältnis der beiden Isotopologe von 1:1, jedoch ähnliche Bandenintensitäten erzielt. Der Deuteriumoxidanteil am Gesamtwasser in den gemischten Expansionen beträgt daher etwa $70 \%$.

Die Messreihen zur synchronen Expansion von $\mathrm{H}_{2} \mathrm{O}$ und $\mathrm{D}_{2} \mathrm{O}$ sind noch nicht abgeschlossen. Daher sollen an dieser Stelle nur die bislang erhaltenen Ergebnisse und verschiedene Ansätze zur Spektreninterpretationen vorgestellt werden. Dieses Kapitel ist somit dem Charakter nach ein Ausblick auf folgende Messungen, ermöglicht jedoch bereits Einsicht in die Anwendungsbreite der Multischlitzdüsenexpansionen. 


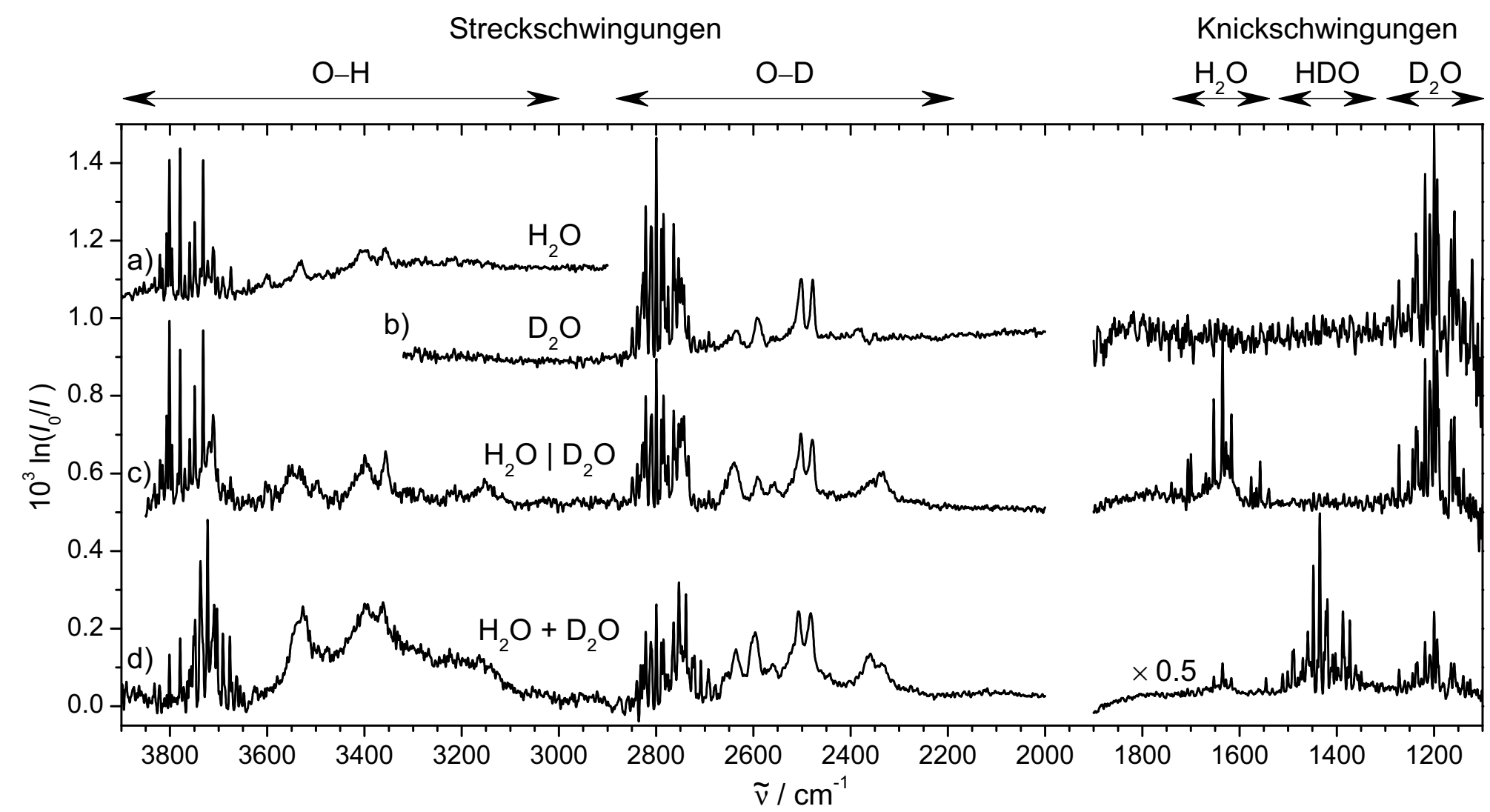

Abbildung 4.11: Übersichtsspektren der Streck- und Deformationsschwingungen von $\mathrm{H}_{2} \mathrm{O}$ und $\mathrm{D}_{2} \mathrm{O}$ in Helium bei der Expansion durch die konvergierende Schlitzdüsenanordnung des Muesli-Jets mit eingezeichneten Spektralbereichen der intramolekularen Fundamentalschwingungen. Düsenabstand $h=2 \mathrm{~cm}, p_{0}=1000$ mbar. a) Expansion von $\mathrm{H}_{2} \mathrm{O}$ durch alle zwanzig Düsen. b) Expansion von $\mathrm{D}_{2} \mathrm{O}$ durch alle zwanzig Düsen. c) Synchrone Expansion von $\mathrm{H}_{2} \mathrm{O}$ und $\mathrm{D}_{2} \mathrm{O}$ durch jeweils die Hälfte der Düsen. d) Expansion von im Reservoir vorgemischtem $\mathrm{H}_{2} \mathrm{O}$ und $\mathrm{D}_{2} \mathrm{O}$ durch alle zwanzig Düsen. 


\begin{tabular}{lcc}
\hline \multirow{2}{*}{ Aggregat } & \multicolumn{2}{c}{$\tilde{\nu} / \mathrm{cm}^{-1}$} \\
& Muesli-Jet & Literatur \\
\hline \hline$\left(\mathrm{H}_{2} \mathrm{O}\right)_{2}$ & 3600 & 3602 \\
$\left(\mathrm{H}_{2} \mathrm{O}\right)_{3}$ & 3533 & 3533 \\
$\left(\mathrm{H}_{2} \mathrm{O}\right)_{4}$ & 3401 & 3401 \\
$\left(\mathrm{H}_{2} \mathrm{O}\right)_{5}$ & 3357 & 3355 \\
$-\left(\overline{\mathrm{D}_{2}}-\overline{\mathrm{O}}\right)_{2}$ & $26 \overline{3} 5$ & $26 \overline{3} 3$ \\
$\left(\mathrm{D}_{2} \mathrm{O}\right)_{3}$ & 2591 & 2591 \\
$\left(\mathrm{D}_{2} \mathrm{O}\right)_{4}$ & 2502 & 2502 \\
$\left(\mathrm{D}_{2} \mathrm{O}\right)_{5}$ & 2478 & 2477 \\
\hline
\end{tabular}

Tabelle 4.6: Experimentelle Bandenzentren stark IR-aktiver Streckschwingungsbanden von $\mathrm{H}_{2} \mathrm{O}$ - und $\mathrm{D}_{2} \mathrm{O}$-Aggregaten aus Überschallexpansionen.

Abbildung 4.11 zeigt die Spektren der Expansionen von $\mathrm{H}_{2} \mathrm{O}$ (a) und $\mathrm{D}_{2} \mathrm{O}$ (b) im Abstand von $2 \mathrm{~cm}$ durch alle zwanzig Schlitzdüsen sowie der synchronen(,,|", c) und vorgemischten $(,+$ ", d) Expansionen beider Substanzen im Bereich der intramolekularen Streck- und Deformationsschwingungen. Die Spektrenlücken zwischen 2000 und $1900 \mathrm{~cm}^{-1}$ sind zum einen auf den zur Spektrenaufnahme verwendeten Sandwich-InSbMCT-Detektor zurückzuführen, bei dem der Übergang zwischen den beiden Detektormaterialien knapp unterhalb von $2000 \mathrm{~cm}^{-1}$ erfolgt, zum anderen aber auch auf die Transmissionsgrenzen eingesetzter Spektralfilter. Bei den Spektren a) und b) der Reinsubstanzen wurden durch Wahl der Konzentrationen Anteile von Hexameren und größeren Aggregaten in den Expansionen vermieden, um Bandenüberlappungen im Spektrum gering zu halten. Die Positionen der stark infrarotaktiven Banden der Aggregate vom Dimer bis zum Pentamer können Tabelle 4.6 entnommen werden. Die Spektren der synchronen Expansionen c) zeigen einen gegenüber den Einzelsubstanzspektren höheren Gesamtwasseranteil, der wie bereits in Abschnitt 3.2.4 erläutert auf die geringeren Nachfüllzeiten bei der Verwendung von zwei Reservoiren zurückzuführen ist. Auch bei einer Vormischung von $\mathrm{H}_{2} \mathrm{O}$ und $\mathrm{D}_{2} \mathrm{O}$ (Abbildung $4.11 \mathrm{~d}$ ) ist die Wasserkonzentration gegenüber den Einzelkomponentenexpansionen a) und b) erhöht, obwohl auch hier nur ein Reservoir verwendet wurde. Dies ist auf die gepulste Gaszufuhr aus den beiden Sättigern zu erklären, da so während des Nachfüllens immer wieder Pausen entstehen, in denen sich die Gasphase im Sättiger mit Wasser anreichern kann. Entsprechend werden in den synchronen und vorgemischten Expansionen auch größere Cluster mit mindestens sechs Wassermolekülen gebildet, wie beispielsweise an den Banden bei 3214 und $3150 \mathrm{~cm}^{-1}$ zu erkennen ist. ${ }^{185} \mathrm{Im}$ O-D-Streckschwingungsbereich wird die Identifizierung von Banden größerer Cluster durch die $\mathrm{CO}_{2}$-Bande zwischen 2400 und $2300 \mathrm{~cm}^{-1}$ erschwert.

Im Bereich der Knickschwingungen kann weder für das reine $\mathrm{D}_{2} \mathrm{O}$ eine Verunreinigung durch HDO festgestellt werden, noch ist die charakteristische HDO-Bande um 


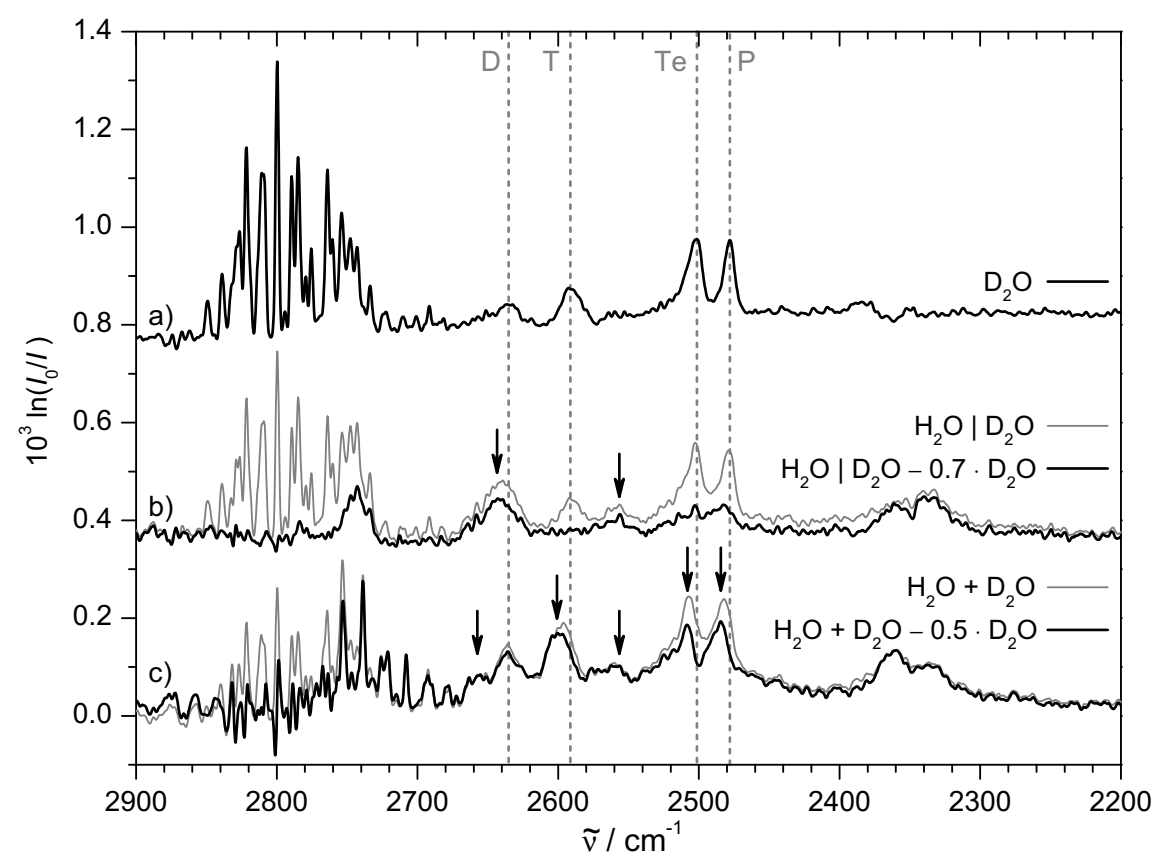

Abbildung 4.12: Differenzspektren im O-D-Streckschwingungsbereich der synchronen (b) und vorgemischten (c) Expansionen von $\mathrm{H}_{2} \mathrm{O}$ und $\mathrm{D}_{2} \mathrm{O}$ aus Abbildung 4.11 nach skalierter Subtraktion des reinen $\mathrm{D}_{2} \mathrm{O}$-Spektrums (a). Die Spektren vor der Differenzbildung sind zusätzlich in grau dargestellt. Pfeile markieren die Positionen zusätzlicher oder verschobener Banden. D: Dimer, T: Trimer, Te: Tetramer, P: Pentamer. Bandenzuordnungen wurden Referenz 5 entnommen. Banden zwischen 2400 und $2300 \mathrm{~cm}^{-1}$ sind zumindest teilweise auf $\mathrm{CO}_{2}$ zurückzuführen.

$1400 \mathrm{~cm}^{-1}$ bei der synchronen Expansion in Spektrum c) mit einer Intensität oberhalb des Rauschlevels zu erkennen. Im Spektrum der vorgemischten Expansion d) stellt sie hingegen wie erwartet die stärkste Bande dar, während die Restintensitäten der $\mathrm{H}_{2} \mathrm{O}$ und $\mathrm{D}_{2} \mathrm{O}$-Knickschwingungen eine statistische Verteilung der Isotopologe bestätigen. Als erstes, wichtiges Ergebnis kann also festgehalten werden, dass in den synchronen Expansionen auch bei einem Abstand von $2 \mathrm{~cm}$ trotz des Vorliegens cyclischer Aggregate kein Isotopenaustausch beobachtet werden kann. Dabei kann an dieser Stelle jedoch noch nicht ausgeschlossen werden, dass die Ursache in der ausbleibenden Bildung gemischter Aggregate liegt.

Hierfür muss eine genauere Betrachtung der Streckschwingungsbereiche wasserstoffbrückengebundener $\mathrm{O}-\mathrm{H}-$ und $\mathrm{O}-\mathrm{D}-\mathrm{Gruppen}$ erfolgen. Da aus Abbildung 4.11 bereits ersichtlich ist, dass sich der O-D-Bereich durch ein höheres Signal-zu-Rauschen-Verhältnis und schmalere Banden auszeichnet, soll dieser hier zuerst betrachtet werden. Vergrößerte Ausschnitte der Spektren der reinen $\mathrm{D}_{2} \mathrm{O}$-Expansion sowie der Differenzspektren der synchronen und vorgemischten Expansionen im O-D-Streckschwingungsbereich nach skalierter Subtraktion des $\mathrm{D}_{2} \mathrm{O}$-Spektrums sind in Abbildung 4.12 gezeigt. 
Zusätzlich sind die Spektren vor der Subtraktion in grau eingezeichnet. Als Referenz für die Differenzbildung diente der $\mathrm{D}_{2} \mathrm{O}$-Monomerbereich. Da die Gesamtwasserkonzentration im reinen $\mathrm{D}_{2} \mathrm{O}$-Spektrum jedoch geringer ist als in den beiden Zweikomponentenspektren, sind in den Differenzspektren insbesondere für größere Cluster Restintensitäten zu erwarten.

So zeigt auch das Spektrum b) der synchronen Expansion von $\mathrm{H}_{2} \mathrm{O}$ und $\mathrm{D}_{2} \mathrm{O}$ nach der Subtraktion eine Bande bei $2740 \mathrm{~cm}^{-1}$, die auf die Streckschwingungen freier O-DGruppen innerhalb von Aggregaten zurückzuführen ist. Während bei den gebundenen O-D-Schwingungen die Bande des Trimers durch die Differenzbildung praktisch vollständig wegfällt, bleiben leichte Restbanden für Tetramere und Pentamere. Bemerkenswerter sind jedoch die große Restintensität im Bereich der Dimerbande, die aufgrund der höheren mittleren Clustergröße relativ zum $\mathrm{D}_{2} \mathrm{O}$-Spektrum a) nicht zu erwarten ist, sowie die zusätzliche Bande bei $2559 \mathrm{~cm}^{-1}$ (Pfeil zwischen Trimer- und Tetramerbande in Abbildung 4.12 b). Es liegt nahe, dass beide Banden die gleiche Ursache besitzen, wobei hierfür sowohl gemischte Aggregate aus $\mathrm{H}_{2} \mathrm{O}$ und $\mathrm{D}_{2} \mathrm{O}$ als auch größere Cluster in Frage kommen. Letztere sollten zusätzliche Banden bei kleineren Wellenzahlen aufweisen ${ }^{186}$, die hier aufgrund der Überlappung mit der antisymmetrischen $\mathrm{CO}_{2}$ Streckschwingungsbande nicht identifiziert werden können.

Differenzspektrum c) der vorgemischten Expansion zeigt deutlich mehr Restbanden. Dies ist einerseits auf den aufgrund des Isotopenaustauschs kleineren Vorfaktor des subtrahierten $\mathrm{D}_{2} \mathrm{O}$-Spektrums zurückzuführen, andererseits aber auch auf die bevorzugte Einbringung der O-D-Gruppen in Wasserstoffbrückenbindungen. ${ }^{184}$ Diese Präferenz lässt sich auch aus Berechnungen der Donor- und Akzeptorstärken analog zu Gleichungen 4.1 und 4.2 erkennen. Auf B3LYP/6-311++ $\mathrm{G}^{* *}$-Niveau ergeben sich für das System $\mathrm{D}_{2} \mathrm{O} / \mathrm{H}_{2} \mathrm{O}$ Werte von $d \approx 1.5$ und $a \approx 0.7$, so dass $\mathrm{D}_{2} \mathrm{O}$ sowohl den stärkeren Donor als auch den besseren Akzeptor darstellt, wobei die Donorstärke überwiegt. Die restliche Monomerintensität in Spektrum 4.12 c ist auf den entkoppelten O-D-Oszillator in freiem HDO zurückzuführen. Die in Spektrum b) beobachtete Bande noch unbekannten Ursprungs bei $2559 \mathrm{~cm}^{-1}$ tritt auch im Spektrum der vorgemischten Expansionen auf, jedoch mit relativ zu den benachbarten Trimer- und Tetramerbanden geringerer Intensität. Dies ist konsistent mit der Theorie, dass ihr ein gemischtes Aggregat aus $\mathrm{H}_{2} \mathrm{O}$ und $\mathrm{D}_{2} \mathrm{O}$ zu Grunde liegt, da diese Cluster in der vorgemischten Expansion eine geringere relative Häufigkeit besitzen, gleiches gilt jedoch auch für große reine $\mathrm{D}_{2} \mathrm{O}$-Cluster. Zusätzlich zeigen sich im Differenzspektrum der vorgemischten Expansionen blauverschobene Schultern (Dimer) bzw. Blauverschiebungen der cyclischen Clusterbanden (Trimer, Tetramer, Pentamer) um etwa $5 \mathrm{~cm}^{-1}$ (Pfeilpositionen in Abbildung 4.12), die in ähnlicher Größenordnung bereits in Edelgasmatrixmessungen für einige gemischte $\mathrm{H}_{2} \mathrm{O} / \mathrm{D}_{2} \mathrm{O} / \mathrm{HDO}$-Trimere gefunden wurden ${ }^{179}$ und möglicherweise auf die entfallende Kopplung der Schwingungen gebundener und freier O-D-Bindungen zurückzuführen sind. Das Fehlen dieser Bandenverschiebungen im Spektrum b) der synchronen Expansionen bestätigt erneut den dort ausbleibenden Isotopenaustausch.

Eine analoge Darstellung des reinen $\mathrm{H}_{2} \mathrm{O}$-Spektrums sowie der beiden Zweikompo- 


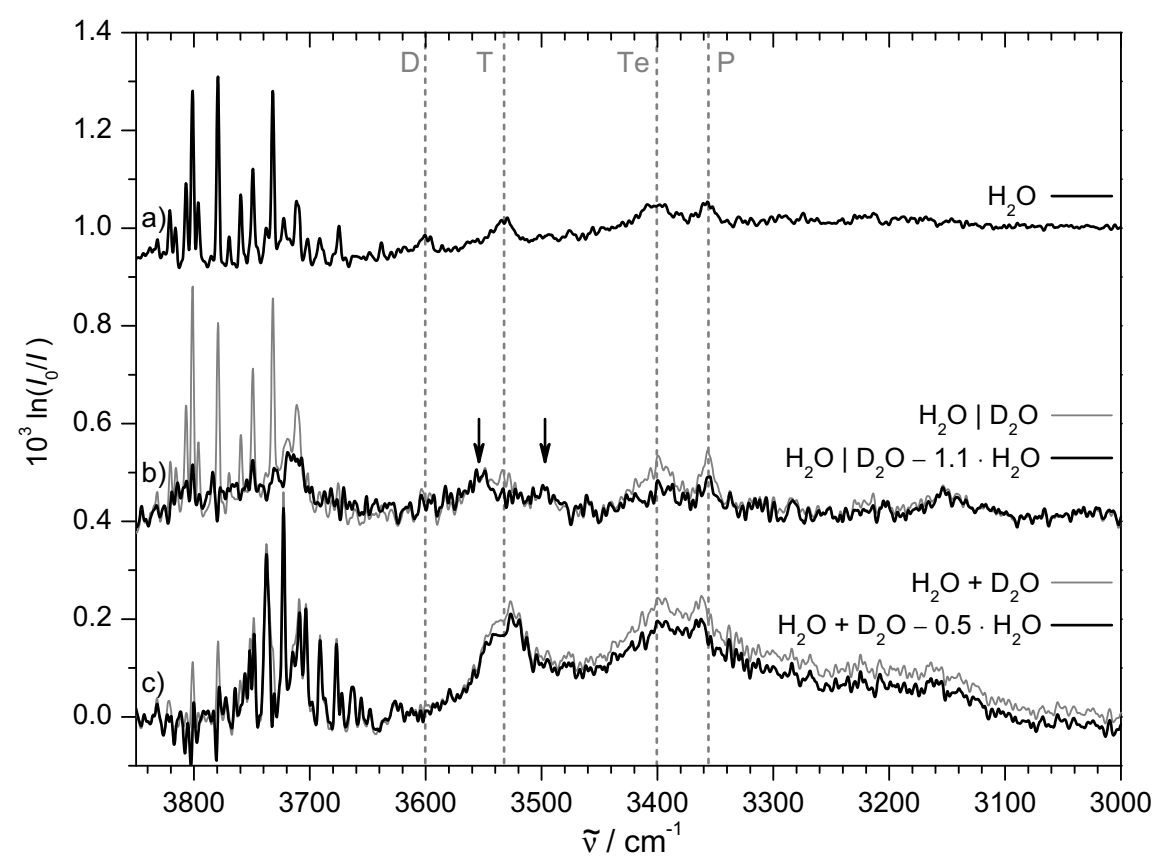

Abbildung 4.13: Differenzspektren im O-H-Streckschwingungsbereich der synchronen (b) und vorgemischten (c) Expansionen von $\mathrm{H}_{2} \mathrm{O}$ und $\mathrm{D}_{2} \mathrm{O}$ aus Abbildung 4.11 nach skalierter Subtraktion des reinen $\mathrm{H}_{2} \mathrm{O}$-Spektrums (a). Die Spektren vor der Differenzbildung sind zusätzlich in grau dargestellt. Pfeile markieren die Positionen zusätzlicher oder verschobener Banden. D: Dimer, T: Trimer, Te: Tetramer, P: Pentamer, Bandenzuordnungen wurden Referenz 5 entnommen.

nenten-Differenzspektren im O-H-Streckschwingungsbereich ist in Abbildung 4.13 gezeigt. Aufgrund des geringeren Signal-zu-Rauschen-Verhältnisses und der schlechteren Bandenseparation ist eine Analyse hier allerdings noch problematischer als im O-DBereich. Im Spektrum der synchronen Expansion b) sind erneut Restbanden in den Bereichen freier Aggregat-O-H-Schwingungen um $3710 \mathrm{~cm}^{-1}$ und der gebundenen Tetramer- und Pentamerschwingungen sowie der größeren Aggregate bei $3150 \mathrm{~cm}^{-1} \mathrm{zu}$ erkennen, die sich wie zuvor aus den unterschiedlichen Gesamtwasserkonzentrationen der zur Differenzbildung herangezogenen Spektren ergeben. Im Gegensatz zum O-DSpektrum in Abbildung $4.12 \mathrm{~b}$ ist im O-H-Bereich bei der synchronen Expansion keine hohe Intensität im Dimerbereich zu erkennen, da $\mathrm{H}_{2} \mathrm{O}$ zum einen in geringerer Konzentration vorliegt und zum anderen bevorzugt als Akzeptor ${ }^{179}$ agiert. Diese Trennung zwischen Donor- und Akzeptorrolle ist in den typischen cyclischen Aggregaten nicht möglich. Zusätzliche Banden relativ zum reinen $\mathrm{H}_{2} \mathrm{O}$-Spektrum sind bei 3554 und eventuell $3497 \mathrm{~cm}^{-1}$ zu erkennen, wobei erstere möglicherweise Wasseraggregaten mit Doppeldonoren, also Wassermolekülen, die in zwei Wasserstoffbrückenbindungen als Donor fungieren, zugeordnet werden können. ${ }^{133}$ Insgesamt ist der O-H-Bereich des $\mathrm{H}_{2} \mathrm{O} \mid \mathrm{D}_{2} \mathrm{O}$-Spektrums somit weniger aussagekräftig als der O-D-Bereich. Er kann bei 


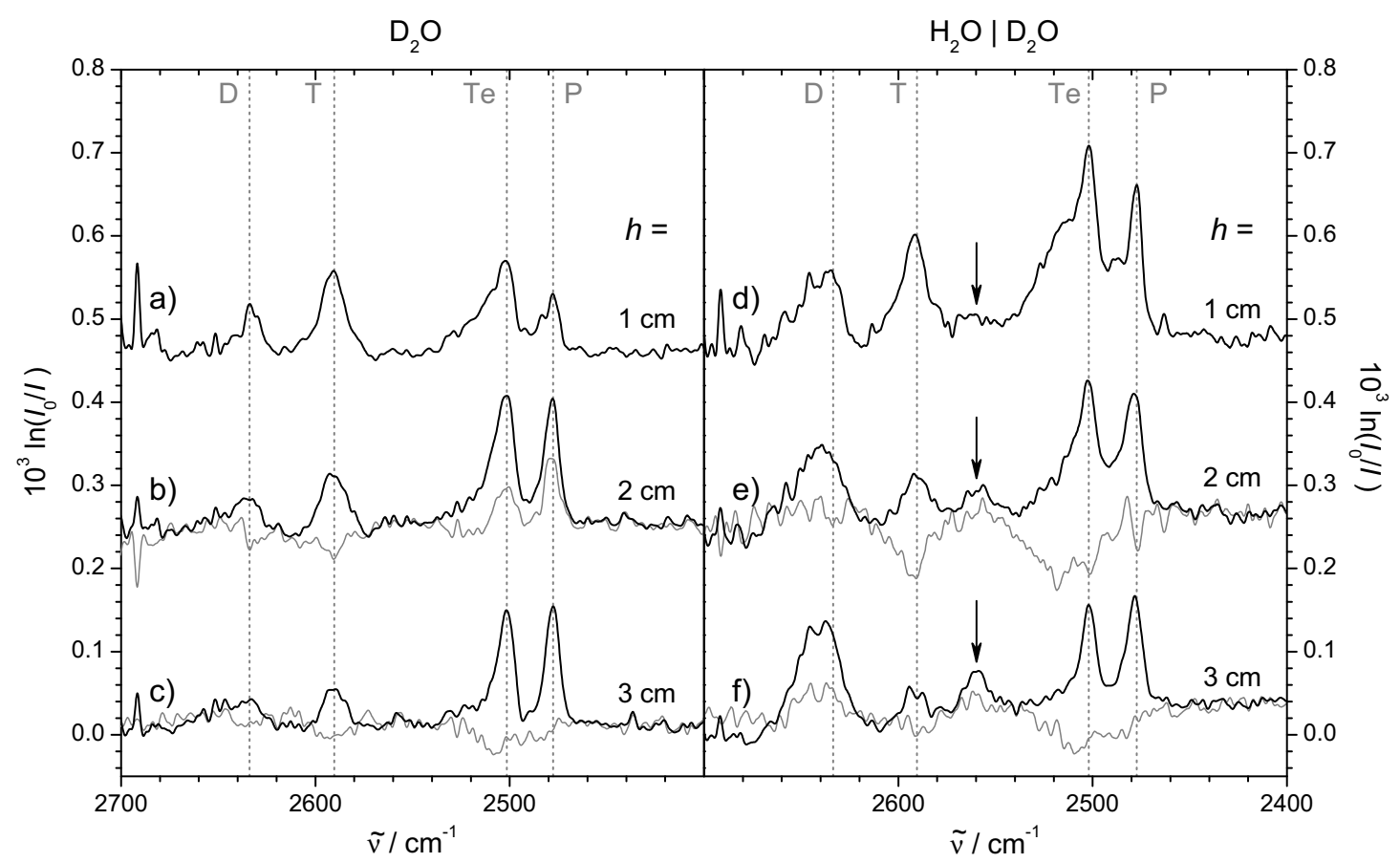

Abbildung 4.14: O-D-Streckschwingungsspektren von Wasserclustern aus Expansionen von Deuteriumoxid in Helium $(\mathrm{a}-\mathrm{c})$ und der synchronen Expansion von $\mathrm{H}_{2} \mathrm{O}$ und $\mathrm{D}_{2} \mathrm{O}$ $(\mathrm{d}-\mathrm{f})$ in verschiedenen Abständen $h$ zum Düsenausgang. Verwendet wurde die konvergierende Düsenanordnung des Muesli-Jets, $p_{0}=1000$ mbar. D, T, Te, P: Positionen der intensiven $\mathrm{D}_{2} \mathrm{O}$ Dimer-, Trimer-, Tetramer- und Pentamer-Absorptionsbanden. Graue Linien für Abstände von 2 und $3 \mathrm{~cm}$ geben die Differenz zum Spektrum bei 1 bzw. $2 \mathrm{~cm}$ an. Pfeile kennzeichnen die Bande unbekannten Ursprungs bei $2559 \mathrm{~cm}^{-1}$.

Interpretationsansätzen, die gemischte Cluster beinhalten, jedoch möglicherweise die notwendigen Zusatzinformationen liefern.

Im Differenzspektrum 4.13 c der vorgemischten Expansion ist zunächst einmal die HDO-Monomerschwingung des $\mathrm{O}-\mathrm{H}$-Oszillators zu erkennen. Insgesamt treten wenige separierte Banden auf, auffällig ist jedoch das Fehlen einer Dimerbande aufgrund der bereits angesprochenen OD-Donorpräferenz. Für Trimere, Tetramere und Pentamere sind jeweils noch einzelne Peaks im Spektrum identifizierbar, diese weisen aber gegenüber den reinen $\mathrm{H}_{2} \mathrm{O}$-Clustern keine erkennbare Verschiebung auf und werden zusätzlich von einem breiten Bandenuntergrund überlagert, der vermutlich von Aggregaten mit mindestens sechs Wassermolekülen in zahlreichen Isotopenkombinationen verursacht wird. Aufgrund dieser Beobachtungen werden sich die weiteren Betrachtungen auf den $\mathrm{O}-\mathrm{D}-$ Streckschwingungsbereich und die synchronen Expansionen von $\mathrm{H}_{2} \mathrm{O}$ und $\mathrm{D}_{2} \mathrm{O}$ konzentrieren.

Zusätzliche Information kann aus einer Gegenüberstellung des Verhaltens der synchronen mit den reinen $\mathrm{D}_{2} \mathrm{O}$-Expansionen bei verschiedenen Abständen zum Düsenaus- 
gang erhalten werden. Abbildung 4.14 zeigt diese Spektren im Bereich der gebundenen O-D-Clusterschwingungen bei Messabständen von 1, 2 und $3 \mathrm{~cm}$ zur Düse. Die Positionen der reinen $\mathrm{D}_{2} \mathrm{O}$-Aggregatbanden sind mit gestrichelten Linien markiert, zusätzlich sind mit steigenden Abständen jeweils die Differenzspektren nach Subtraktion des vorangegangenen Spektrums in grau eingezeichnet. Die Bande unbekannten Ursprungs bei $2559 \mathrm{~cm}^{-1}$ ist mit Pfeilen markiert.

In den Spektren der $\mathrm{D}_{2} \mathrm{O}$-Expansionen links zeigt sich bei einer Abstanderhöhung von 1 auf $2 \mathrm{~cm}$ ein Intensitätsabfall für die Dimer- und Trimerbanden, während die Bandenintensitäten der Tetramere und Pentamere steigen. Dies ist auf die Kombination von Dichteabfall und verzögerter Bildung größerer Aggregate in der Expansion zurückzuführen. Die gleichzeitig abnehmenden Bandenbreiten weisen auf die zunehmende Abkühlung hin. In einem weiter erhöhten mittleren Abstand von $3 \mathrm{~cm}$ zur Düse sind für die verschiedenen Clustergrößen kaum Unterschiede zu erkennen, alle Banden verlieren aufgrund des Dichteabfalls an Intensität und eine weitere leichte Reduktion der Bandenbreiten zeigt die weiter sinkende Temperatur an.

Im Vergleich zu der reinen $\mathrm{D}_{2} \mathrm{O}$-Expansion a) zeigt das Spektrum $4.14 \mathrm{~d}$ der synchronen Expansionen von $\mathrm{H}_{2} \mathrm{O}$ und $\mathrm{D}_{2} \mathrm{O}$ bei einem Messabstand von $1 \mathrm{~cm}$ die bereits beobachteten größeren Clusterkonzentrationen. Auffällig ist dabei insbesondere die relativ hohe Dimerintensität, die bei einer allgemein höheren mittleren Clustergröße zunächst nicht zu erwarten ist ${ }^{133}$ und mit steigendem Abstand zusätzlich an Intensität gewinnt. Zusammen mit der hohen Halbwertsbreite der Dimerbande weist dies erneut auf eine Überlagerung mit anderen Banden aus größeren Clustern oder gemischten Aggregaten hin. Die ausgeprägten blauverschobenen Schultern der Tetramer- und Pentamerbanden bei kleinen Abständen zur Düse (Spektrum d) sind hingegen eher auf Anteile warmer Aggregate (siehe auch Kapitel 4.2.2) zurückzuführen, da sie bei größeren Düsenabständen nicht mehr auftreten. Im Abstand von $2 \mathrm{~cm}$ zur Düse zeigt sich wie beim reinen $\mathrm{D}_{2} \mathrm{O}$ eine abnehmende Halbwertsbreite der Banden durch zunehmende Abkühlung. Während die durch den Dichteabfall zu erwartende sinkende Intensität für Trimer und Tetramer beobachtet werden kann, wachsen die Banden des Dimers und Pentamers, zusätzlich wird die Bande bei $2559 \mathrm{~cm}^{-1}$ nun deutlich erkennbar. Als Ursache für diesen Intensitätsanstieg kommen zum einen eine hohe Clustergröße und somit verzögerte Bildung innerhalb der Expansion infrage, wie sie beim Pentamer vorliegt, oder aber möglicherweise eine gemischte Struktur aus $\mathrm{H}_{2} \mathrm{O}$ und $\mathrm{D}_{2} \mathrm{O}$, da diese erst in der Expansion aufeinandertreffen und so die Aggregationsprozesse ebenfalls erst später abgeschlossen sein könnten. Während bei einem Übergang vom $2 \mathrm{~cm}$ - auf das $3 \mathrm{~cm}$-Spektrum bei den reinen $\mathrm{D}_{2} \mathrm{O}$-Expansionen nur noch der Dichteabfall sichtbar ist (Spektren $4.14 \mathrm{~b}$ und c), kann in den synchronen Expansionen weiterhin ein Anstieg im Dimerbereich und um $2559 \mathrm{~cm}^{-1}$ beobachtet werden. Sollte die Ursache für diese Banden(überlappungen) in größeren, reinen $\mathrm{D}_{2} \mathrm{O}$-Aggregaten begründet liegen, müssen diese mindestens sechs Moleküle enthalten, da für die Pentamerbande die Intensität zwischen $2 \mathrm{~cm}$ und $3 \mathrm{~cm}$ sinkt.

Die Herkunft dieser beiden Banden bei 2645 und $2559 \mathrm{~cm}^{-1}$ ist also zu diskutieren. In 
früheren FTIR-Spektren der Einfachschlitzdüsenexpansionen von $\mathrm{D}_{2} \mathrm{O}$ in Argon kann bei steigender Deuteriumoxidkonzentration ebenfalls ein auffälliger Anstieg von Banden bei 2640 und $2560 \mathrm{~cm}^{-1}$ beobachtet werden (siehe Referenz 107, S. 307). Dies kann einerseits als Hinweis auf einen Ursprung in großen, reinen $\mathrm{D}_{2} \mathrm{O}-$ Clustern gesehen werden. Andererseits werden diese Banden in den Argon-Expansionen von weiteren Peaks bei etwa 2400,2380,2330, 2430 und $2250 \mathrm{~cm}^{-1}$ begleitet, die teilweise nicht von der $\mathrm{CO}_{2}$-Bande überlagert sein dürften und dennoch in Abbildung $4.12 \mathrm{~b}$ nicht zu erkennen sind. Zudem führt im Spektrum aus Referenz 107 die Bildung großer Aggregate zu einem deutlich erkennbaren, breiten Untergrund im Spektrum zwischen 2650 und $2200 \mathrm{~cm}^{-1}$, der hier ebenfalls nicht auftritt. Untersuchungen von Deuteriumoxid-Helium-Expansionen durch Infrarot-Cavity-Ringdown-Laserabsorptionsspektroskopie zeigten ebenfalls Banden bei 2640 und $2570 \mathrm{~cm}^{-1}$, die dort Aggregaten mit mindestens 7 $\mathrm{D}_{2} \mathrm{O}-$ Molekülen zugeordnet wurden ${ }^{186}$, auch hier fehlt jedoch die zusätzlich gefundene Bande bei $2450 \mathrm{~cm}^{-1}$ in den Spektren in Abbildung 4.14 .

Die Literaturspektren weisen somit auf einen möglichen Ursprung der Banden bei 2645 und $2559 \mathrm{~cm}^{-1}$ in großen, reinen $\mathrm{D}_{2} \mathrm{O}$-Clustern hin, wobei hierbei von Aggregaten mit mehr als sechs $\mathrm{D}_{2} \mathrm{O}$ Molekülen auszugehen ist, da die charakteristische Bande der im Jet bevorzugt gebildeten Käfig-Hexamerstruktur ${ }^{145,187}$ bei $2395 \mathrm{~cm}^{-1186}$ nicht beobachtet werden kann. Dabei ist allerdings zu beachten, dass sich auch die O-DStreckschwingungen größerer Aggregate mit einzelnen $\mathrm{H}_{2} \mathrm{O}$-Molekülen nicht signifikant von denen reiner $\mathrm{D}_{2} \mathrm{O}$-Cluster unterscheiden dürften. Zumindest bieten sowohl die Methanol-tert-Butylalkohol-Spektren in Abschnitt 4.1.1 als auch die schwache Dimerbande im O-H-Streckschwingungsbereich des Spektrums bei synchroner Expansion von $\mathrm{H}_{2} \mathrm{O}$ und $\mathrm{D}_{2} \mathrm{O}$ in Abbildung $4.13 \mathrm{~b}$ Anhaltspunkte für eine Bildung gemischter Aggregate, so dass auch die Streckschwingungsbanden kleinerer gemischter Cluster in den Spektren auftreten sollten.

Da der Muesli-Jet keine Möglichkeit der massenselektiven Aufnahme von Infrarotspektren ${ }^{188}$ bietet, kann an dieser Stelle keine Bandenzuordnung für gemischte Aggregate erfolgen. Weitere Spektren bei geringeren Wasserkonzentrationen und anderen $\mathrm{H}_{2} \mathrm{O} / \mathrm{D}_{2} \mathrm{O}$-Verhältnissen könnten dennoch für die Bandeninterpretation aufschlussreich sein. Um die Suche nach gemischten $\mathrm{H}_{2} \mathrm{O}-\mathrm{D}_{2} \mathrm{O}$-Aggregaten zu unterstützen und infrage kommende kleine Strukturen für die Banden bei 2645 und $2559 \mathrm{~cm}^{-1} \mathrm{zu}$ identifizieren sollen hier berechnete Spektren für verschiedene Aggregatgrößen und -strukturen vorgestellt werden.

Bei früheren Rechnungen zu den Normalschwingungen von $\mathrm{H}_{2} \mathrm{O}-\mathrm{D}_{2} \mathrm{O}$-Aggregaten hat sich die DFT-Methode B3LYP/6-311++ $\mathrm{G}^{* *}$ als geeigneter Kompromiss zwischen guter Beschreibung und Rechenaufwand erwiesen. ${ }^{5}$ Dies ist eher auf Effekte der Fehlerkompensation als auf die Realitätsnähe der Methode zurückzuführen, was für die qualitativen Abschätzungen an dieser Stelle jedoch nicht von Belang sein soll. Da Aggregate mit mindestens sechs Wasser-Molekülen delokalisierte Schwingungen durch Kopplungen zwischen intra- und intermolekularen Moden aufweisen ${ }^{146}$, zeigen sie eine große Anzahl an Schwingungen, die teils mit denen kleinerer Cluster überlappen. 
Aufgrund der typischen Abweichungen zwischen gemessenen und harmonisch berechneten Spektren von Wasserclustern kann hier für derartige größere Systeme ohnehin keine zuverlässige Bandenzuordnung erfolgen. Daher werden folgende Messreihen mit geringeren Wasserkonzentrationen durchgeführt und die hier vorgestellten Berechnungen konzentrieren sich auf gemischte Wasseraggregate mit höchstens fünf Molekülen. Da das Knickschwingungsspektrum der synchronen Expansion von $\mathrm{H}_{2} \mathrm{O}$ und $\mathrm{D}_{2} \mathrm{O}$ in Abbildung $4.11 \mathrm{c}$ keine Anzeichen für die Bildung von HDO lieferte, werden hierbei nur Cluster aus $\mathrm{H}_{2} \mathrm{O}$ und $\mathrm{D}_{2} \mathrm{O}$ berücksichtigt.

Betrachtet werden sollen die typischen globalen Minimumstrukturen vom Dimer bis zum Pentamer, bei denen Trimer, Tetramer und Pentamer cyclisch vorliegen. Aufgrund der gegenüber Einfachschlitzdüsenexpansionen höheren Temperaturen und den noch nicht bekannten Bildungsmechanismen gemischter Aggregate mit der konvergierenden Schlitzdüsenanordnung sollen jedoch auch atypischere Strukturen untersucht werden (siehe Abbildung 4.15). Hierbei handelt es sich zum einen um offenkettige Trimere, wie sie in den warmen Expansionen in Abschnitt 4.2.2 gefunden wurden. Da $\mathrm{D}_{2} \mathrm{O}$ und $\mathrm{H}_{2} \mathrm{O}$ keine identischen Donor- und Akzeptorstärken aufweisen ${ }^{184}$, werden zum anderen offene Trimerstrukturen, bei denen das mittlere Molekül entweder als zweifacher Donor oder zweifacher Akzeptor fungiert, untersucht. Eine günstige Anordnung der Monomer-Isotopologe kann hierbei eine leichte Reduktion der für reine $\mathrm{H}_{2} \mathrm{O}$ - und $\mathrm{D}_{2} \mathrm{O}$ Aggregate berechneten Energiedifferenz von etwa $22-25 \mathrm{~kJ} \mathrm{~mol}^{-1}$ gegenüber den Minimumstrukturen bewirken und so zur Bildung in wärmeren Expansionen beitragen. Da außerdem bereits vor der Durchmischung der Expansionen eine Bildung von Homoaggregaten stattfinden kann, ist auch eine Bildung von Mischaggregaten aus zwei je isotopeneinheitlichen Aggregaten oder aus einem Aggregat und einem zusätzlichen Monomer denkbar. Eine so gebildete Struktur aus einem cyclischen Trimer mit einer angelagerten Seitenkette wäre vermutlich aufgrund der Ringspannung nicht stabil. „Lassoförmige“ Aggregate aus einem Tetramer und (beispielsweise) einem Monomer sind zwar wahrscheinlicher und wurden bereits für Alkohole mit sperrigen Alkylketten wie dem tert-Butylalkohol diskutiert ${ }^{115}$, müssten allerdings entsprechend auch in den reinen $\mathrm{D}_{2} \mathrm{O}$-Expansionen auftreten. Im Gegensatz zu den Alkoholen ist bei Wasser eine Orientierung des Seitenkettenmonomers sowohl als Donor als auch als Akzeptor möglich.

Abbildung 4.15 zeigt auf der linken Seite das Übersichtsspektrum der synchronen Expansion von $\mathrm{H}_{2} \mathrm{O}$ und $\mathrm{D}_{2} \mathrm{O}$ aus Abbildung $4.11 \mathrm{c}$ im Vergleich zu den Bandenbereichen einiger der angesprochenen Aggregattypen. Die gekennzeichneten Bereiche wurden dabei auf Basis der harmonischen B3LYP/6-311++ $\mathrm{G}^{* *}$-Wellenzahlen erhalten. Die Skalierungsfaktoren für jede Clustergröße wurden anhand der stabilsten cyclischen $\mathrm{H}_{2} \mathrm{O}$ - und $\mathrm{D}_{2} \mathrm{O}$-Strukturen erhalten, indem die jeweils intensivste berechnete Streckschwingungsbande mit der entsprechenden experimentellen Bandenposition aus Tabelle 4.6 in Übereinstimmung gebracht wurde. Dabei wurden Streckschwingungsbanden mit einer Intensität von mindestens $50 \mathrm{~km} \mathrm{~mol}^{-1}$ aller möglichen Kombinationen der beiden Wasserisotopologe in den Clustern berücksichtigt. Eine Ausnahme hiervon 


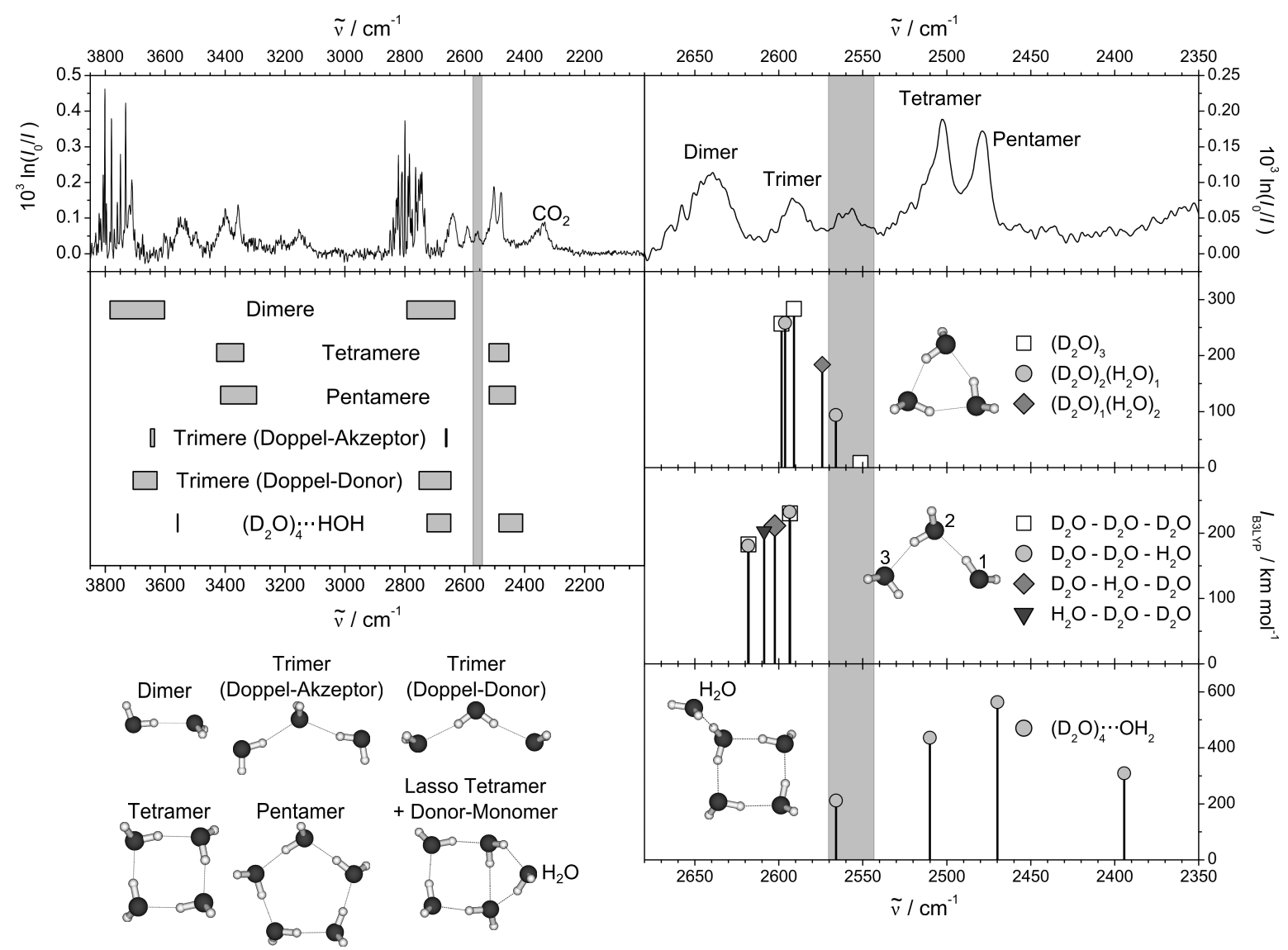

Abbildung 4.15: Vergleich experimenteller Spektren der synchronen Expansion von $\mathrm{H}_{2} \mathrm{O}$ und $\mathrm{D}_{2} \mathrm{O}$ aus Abbildung $4.11 \mathrm{c}$ mit berechneten Wellenzahlen verschiedener gemischter Aggregate von $\mathrm{H}_{2} \mathrm{O}$ und $\mathrm{D}_{2} \mathrm{O}$ auf B3LYP/6-311++G**-Niveau. Theoretische Wellenzahlen sind auf die Übereinstimmung der analogen globalen Minimumstrukturen reiner $\mathrm{H}_{2} \mathrm{O}$ - bzw. $\mathrm{D}_{2} \mathrm{O}$-Aggregate mit den experimentellen intensiven Streckschwingungsbanden skaliert. Links: Übersicht und Schwingungsbereiche von Aggregaten ohne Übereinstimmung mit der bislang nicht zugeordneten Bande bei $2559 \mathrm{~cm}^{-1}$. Rechts: Ausschnitt der O-D-Aggregatstreckschwingungen und Linienspektren ausgewählter Strukturen. D: Dimer, T: Trimer, Te: Tetramer, P: Pentamer, Bandenpositionen aus Tabelle 4.6. Weitere Informationen zu unskalierten Wellenzahlen, Intensitäten und Skalierungsfaktoren $f$ befinden sich im Anhang A.4. 
stellt die Lasso-Struktur $\left(\mathrm{D}_{2} \mathrm{O}\right)_{4} \cdots \mathrm{HOH}$ dar, bei der als Referenz für die Skalierung das cyclische $\mathrm{D}_{2} \mathrm{O}$-Tetramer verwendet wurde. Aufgrund des $\mathrm{D}_{2} \mathrm{O}$-Überschusses in der Expansion und des angenommenen Bildungsmechanismus wurden hier außerdem keine weiteren Isotopomere und Isotopologe dieses Aggregats mit einbezogen.

Der für die Dimere eingezeichnete Bereich kommt durch die Berücksichtigung sowohl der gebundenen als auch der freien $\mathrm{O}-\mathrm{H}$ - und $\mathrm{O}-\mathrm{D}$-Streckschwingungen zustande, hierbei ist der Einfluss des Akzeptorisotopologs auf die Donorschwingung nur gering. Bei den cyclischen Tetrameren zeigt sich, dass das Einbringen von Fremdisotopologen in den Ring lediglich geringfügige Verschiebungen, überwiegend zu kleinen Wellenzahlen, hervorruft. Dies entspricht allerdings nicht den experimentellen Ergebnissen für die gemischten Tetramere in Neon-Matrizen ${ }^{185}$, die im O-D-Streckschwingungsbereich eher blauverschobene Banden zwischen 2508 und $2490 \mathrm{~cm}^{-1}$ (relativ zu $2492 \mathrm{~cm}^{-1}$ für $\left.\left(\mathrm{D}_{2} \mathrm{O}\right)_{4}\right)$ liefern. Ein ähnliches Verhalten wie bei den Tetrameren sagen die Rechnungen auch für cyclische Pentamere voraus. Die Schwingungen der Trimere mit mittlerem Doppel-Donor- oder -Akzeptormolekül stimmen in etwa mit den Bereichen der Dimerschwingungen überein, so dass derartige Bindungsmotive - wenn auch vermutlich in größeren Aggregaten ${ }^{133}$ - unter Umständen zu der hohen Intensität nahe der Dimerbande in den Spektren der synchronen Expansionen um $2645 \mathrm{~cm}^{-1}$ beitragen. Die Lassostruktur mit einzelnem $\mathrm{H}_{2} \mathrm{O}$-Donormolekül besitzt Ähnlichkeit mit der des cyclischen Pentamers, was sich auch in den berechneten Schwingungswellenzahlen widerspiegelt. Andererseits enthält sie auch dreifach koordinierte Moleküle (Donor-DonorAkzeptor und Donor-Akzeptor-Akzeptor), so dass sich zusätzlich Schwingungswellenzahlen ähnlich derer der zuvor besprochenen Donor-Donor- bzw. Akzeptor-AkzeptorTrimere ergeben. Für die Deutung der Bande bei $2559 \mathrm{~cm}^{-1}$ sind alle diese Strukturen voraussichtlich nicht relevant.

Die rechte Seite von Abbildung 4.15 zeigt einen Ausschnitt des experimentellen Spektrums auf der linken Seite im Bereich der O-D-Streckschwingungen der kleinen Cluster vom Dimer bis zum Pentamer. Zum Vergleich sind die theoretischen Linienspektren einzelner Strukturen gezeigt, die für eine Interpretation der Bande bei $2559 \mathrm{~cm}^{-1}$ von Bedeutung sein könnten. Die Skalierung der Banden ist wie zuvor an den intensiven O-D-Streckschwingungen der jeweiligen stabilsten Aggregate orientiert.

Koexpansionen von Methanol und Monodeuteromethanol $\left(\mathrm{CH}_{3} \mathrm{OD}\right)$ haben für gemischte Trimere der beiden Methanol-Isotopologe im O-H-Streckschwingungsbereich eine Rotverschiebung der intensiven Streckschwingungsbanden um etwa 20 und $35 \mathrm{~cm}^{-1}$ gegenüber der des $\left(\mathrm{CH}_{3} \mathrm{OH}\right)_{3}$-Trimers gezeigt. Somit stellen gemischte Trimere auch eine mögliche Ursache für die im O-D-Bereich der Wasserspektren beobachtete Bande bei $2559 \mathrm{~cm}^{-1}$ (zwischen denjenigen der Trimere und der Tetramere) dar. Zudem stimmt die Bandenposition von $2559 \mathrm{~cm}^{-1}$ mit der der ramanaktiven Trimerbande ${ }^{5}$ von $\left(\mathrm{D}_{2} \mathrm{O}\right)_{3}$ überein, was eine Infrarotaktivierung der Bande in den gemischten, symmetrieärmeren Trimeren vermuten lässt. Allerdings zeigen die Rechnungen für die gemischten Trimere ausschließlich Banden bei deutlich höheren Wellenzahlen. Das erhaltene Bandenmuster wird außerdem durch das der analogen Fluorwasserstofftrimere bestätigt. ${ }^{189,190}$ Das 
gemischte Trimer $\left(\mathrm{D}_{2} \mathrm{O}\right)_{1}\left(\mathrm{H}_{2} \mathrm{O}\right)_{2}$ ist als Verursacher der Bande bei $2559 \mathrm{~cm}^{-1}$ einerseits aufgrund des höheren $\mathrm{D}_{2} \mathrm{O}$-Anteils in der Expansion unwahrscheinlich, andererseits aber auch aufgrund von Messungen in der Argon- beziehungsweise Krypton-Matrix, in denen für diesen Cluster Wellenzahlen von etwa $2571 \mathrm{~cm}^{-1}$ (im Vergleich zu $2578 \mathrm{~cm}^{-1}$ für $\left.\left(\mathrm{D}_{2} \mathrm{O}\right)_{3}\right)$ erhalten wurden. ${ }^{191}$ Für das deuteriumreichere Trimer $\left(\mathrm{D}_{2} \mathrm{O}\right)_{2}\left(\mathrm{H}_{2} \mathrm{O}\right)_{1}$ wurden an gleicher Stelle Wellenzahlen um 2579 und $2557 \mathrm{~cm}^{-1}$ erhalten, die zunächst grob zu den Jet-Banden bei $2591\left(\mathrm{D}_{2} \mathrm{O}\right.$-Trimer $)$ und $2559 \mathrm{~cm}^{-1}$ zu passen scheinen. Allerdings treten sie in einem Intensitätsverhältnis von 3:1 auf, das insbesondere mit dem Spektrum der synchronen Expansionen im Abstand von $3 \mathrm{~cm}$ in Abbildung $4.14 \mathrm{f}$ nicht vereinbar ist. Dagegen spricht auch die Wellenzahldifferenz von $14 \mathrm{~cm}^{-1}$ für die intensive $\left(\mathrm{D}_{2} \mathrm{O}\right)_{3}$-Bande in Jet und Matrix, die vermutlich auch entsprechend im gemischten Aggregat auftreten wird, so dass die Wellenzahlübereinstimmung nur mäßig ist. Auch die Auswirkung der Verteilung der Wasserisotopologe in den gemischten Trimeren bezüglich der Ausrichtung der freien Wasserstoffatome ${ }^{192}$ ist laut Rechnungen mit etwa $0.1 \mathrm{~cm}^{-1}$ vernachlässigbar.

Das Auftreten ungewöhnlicher Effekte im Bereich der Trimerbanden in Kombination mit einer hohen Intensität im Dimerbereich lässt eine Parallele zur Diskussion offenkettiger Trimerstrukturen in Kapitel 4.2.2 vermuten. Aufgrund der etwas höheren Donorstärke von $\mathrm{D}_{2} \mathrm{O}$ gegenüber $\mathrm{H}_{2} \mathrm{O}^{184}$ könnten derartige Strukturen selbst bei niedrigeren Expansionstemperaturen als den dort betrachteten begünstigt sein. In Abbildung 4.15 sind aufgrund des Isotopenverhältnisses in der Expansion nur die berechneten Banden offenkettiger $\left(\mathrm{D}_{2} \mathrm{O}\right)_{2}\left(\mathrm{H}_{2} \mathrm{O}\right)_{1}$-Trimere bei verschiedener Anordnungsreihenfolge der Moleküle sowie des offenen reinen $\mathrm{D}_{2} \mathrm{O}$-Trimers gezeigt. Die angegebene Molekülreihenfolge richtet sich dabei vom Donor- zum Akzeptormolekül. Die Strukturen wurden durch Fixierung eines O-O-Abstands auf $4 \AA$ erhalten. Wie bereits bei den $\mathrm{H}_{2} \mathrm{O}$-Trimeren beobachtet, entspricht auch für $\mathrm{D}_{2} \mathrm{O}$ die Position der intensivsten Streckschwingung des offenen Trimers recht genau der der experimentellen Bande des analogen cyclischen Aggregats. Sämtliche Banden der gemischten Aggregate weisen höhere Wellenzahlen auf und fallen somit ebenfalls nicht in den hier interessanten Bereich. Dabei muss allerdings beachtet werden, dass die Wellenzahlen eine deutliche Abhängigkeit vom $\mathrm{O}-\mathrm{O}-\mathrm{O}-$ Winkel und den O-O-Abständen aufweisen, sodass für verlässliche Aussagen weitere Rechnungen unter Variation des Winkels vonnöten wären, die vor der Aufnahme weiterer Messreihen den Rahmen des Sinnvollen übersteigen.

Die Geometrie der Expansionen lässt die zumindest intermediäre Bildung von Lassostrukturen nicht unwahrscheinlich erscheinen. Zudem zeigt das Übersichtsspektrum links in Abbildung 4.15, dass für $\mathrm{H}_{2} \mathrm{O}$ die Monomerkonzentration recht hoch ist, während $\mathrm{D}_{2} \mathrm{O}$ aufgrund des höheren Anteils in der Expansion mehr Aggregate bildet, bei denen das Tetramer in der Intensität dominierend ist. Obwohl $\mathrm{D}_{2} \mathrm{O}$ ein besserer Donor als Akzeptor ist, ist die rechts in der Abbildung gezeigte Lassostruktur gegenüber der verbrückenden Struktur links laut Rechnungen um etwa $6 \mathrm{~kJ} \mathrm{~mol}^{-1}$ und gegenüber einem cyclischen Pentamer $\left(\mathrm{D}_{2} \mathrm{O}\right)_{4}\left(\mathrm{H}_{2} \mathrm{O}\right)_{1}$ um $16 \mathrm{~kJ} \mathrm{~mol}^{-1}$ benachteiligt. Das simulierte Spektrum weist zwar sowohl eine Bande im für die $2559 \mathrm{~cm}^{-1}$-Bande interessanten Be- 
reich als auch zwei Banden im Tetramer/Pentamer-Bereich auf, jedoch auch noch eine relativ intensive, weiter rotverschobene Bande, für die das experimentelle Spektrum keine klare Entsprechung besitzt. Somit liefert auch dieses Aggregat keine überzeugende Erklärung.

Insgesamt bestätigen die Berechnungen die Notwendigkeit zusätzlicher Messungen für die Identifikation gemischter $\mathrm{H}_{2} \mathrm{O}-\mathrm{D}_{2} \mathrm{O}$-Aggregate in den Muesli-Jet-Expansionen, auch wenn sie sicherlich keine quantitativ verwertbaren Resultate oder gar ein vollständiges Bild der potentiell in den Expansionen gebildeten Strukturen liefern. Bisherige Ergebnisse für die Banden in den Spektren der synchronen Expansionen, die zusätzlich zu den Reinsubstanzspektren beobachteten auftreten, favorisieren eine Zuordnung zu großen (isotopenreinen oder gemischten) Aggregaten. Sowohl die Ergebnisse der gemischten Alkoholspektren in Kapitel 4.1.1 als auch die schwache O-H-Dimerdonorbande in den synchronen Expansionen lassen jedoch auch eine ausbleibende Bildung gemischter Aggregate unwahrscheinlich erscheinen. Die Rechnungen zeigen, dass für den Nachweis gemischter Aggregate das Trimer am geeignetsten ist, die zugehörigen Banden werden in den bislang gemessenen Spektren jedoch möglicherweise durch die größerer Cluster überlagert. Weitere Messungen mit geringeren Konzentrationen und anderen Isotopenverhältnissen sind somit notwendig und auch eine Ausweitung der Untersuchungen auf Raman-spektroskopische Messungen erscheint aufgrund der gegenüber den FTIR-Spektren unterschiedlichen Schwingungsbandenintensitäten sinnvoll.

\subsection{Zusammenfassung und Ausblick}

Die mit dem Multischlitzdüsenaufbau aufgenommenen und hier vorgestellten Spektren haben gezeigt, dass sich hiermit sowohl ungewöhnlich warme Expansionen als auch die Koexpansionen nicht vormischbarer Substanzen realisieren lassen. Durch diese Fähigkeiten hebt sich der Muesli-Jet von vergleichbaren Einfachschlitzdüsenaufbauten ab. Zudem besitzt er den Vorteil der Variierbarkeit der Schlitzanordnung im Bezug auf Winkel, Anzahl, Abstände und Schlitzdimensionen, so dass die Düsengeometrie mit vergleichsweise geringem Aufwand an die Problemstellung angepasst werden kann.

Die Messungen haben gezeigt, dass so sowohl die Strukturaufklärung von Heteroaggregaten - sogar unter Unterdrückung von Isotopenaustauschreaktionen - ermöglicht wird als auch die Erforschung des Grenzgebiets zwischen Expansions- und Gasphasenbedingungen. Letztes kann sich insbesondere als interessant für den Themenkreis der Atmosphärenchemie erweisen.

Folgende Messungen sollten sich weiter mit dem System $\mathrm{H}_{2} \mathrm{O} / \mathrm{D}_{2} \mathrm{O}$ beschäftigen, da hier bei ausbleibendem Isotopenaustausch eine deutliche Vereinfachung der strukturellen Vielfalt innerhalb der Expansion gegenüber vorgemischten Systemen erzielt werden kann. Neben Variationen der eingesetzten Wasserkonzentrationen könnten auch Messungen des verwandten, jedoch weniger komplexen Systems Methanol/Methanol-D1 in Kombination mit den bekannten Kopplungsmatrixelementen der Isotopologe von 
Methanol ${ }^{105}$ und Wasser ${ }^{5}$ die Identifikation von Schwingungsbanden gemischter Wasseraggregate unterstützen.

Aufgrund der breiten Einsetzbarkeit des Muesli-Jets, die nur durch den Dampfdruck und die Infrarotaktivität der verwendeten Substanzen limitiert ist, wurde er so zu einer universellen Methode zur Untersuchung der intermolekularen Wechselwirkungen gemischter und thermisch angeregter Aggregate weiterentwickelt. 


\section{Donor-Akzeptor-Präferenzen}

Wie im vorhergegangenen Kapitel gezeigt, besitzt Wasser für die Ausbildung von Wasserstoffbrückenbindungen je zwei Donor- und Akzeptorfunktionalitäten. Diese stellen die Grundlage für die cyclischen Strukturen der kleinen Aggregate vom Trimer bis zum Pentamer und die vernetzten Strukturen ab dem Hexamer sowie für das flüssige Vorliegen bei Standardbedingungen dar. ${ }^{3,123,193}$ Flüssiges Wasser ist für viele Substanzen ein sehr gutes Lösungsmittel, wobei bei der Solvatation eine Restrukturierung des Wasserstoffbrückennetzwerks des Wassers bei gleichzeitiger Optimierung der intermolekularen Wechselwirkungen mit den gelösten Molekülen stattfindet. Als „bevorzugtes Solvens der Natur" 194 sind insbesondere die Interaktionen von Wasser mit biologisch relevanten Substanzen von Bedeutung, wobei nicht nur das makroskopische Verhalten in einer wässrigen Umgebung, sondern auch die Betrachtung der Mikrohydratation, also der Anlagerung einzelner Wassermoleküle, von Interesse ist. Ihre Relevanz wurde bereits für Prozesse wie die molekulare Erkennung, die enzymatische Katalyse und den Ladungstransfer gezeigt, bei denen die Art der Anbindung einzelner Wassermoleküle entscheidend für den Reaktionsmechanismus sein kann. 7,8,194,195,196

Isolierte Molekülcluster, wie sie in Überschallexpansionen generiert werden, können geeignete Modellsysteme für die Untersuchung der Wasserstoffbrückenbindungsmuster solcher mikrosolvatisierter Systeme sein. Dies gilt insbesondere, da in Röntgenbeugungsexperimenten die Detektion von Wasserstoffatomen durch deren kleine Streuamplituden erschwert wird. Für Gasphasenuntersuchungen sind hingegen die oft geringen Dampfdrücke von Biomolekülen problematisch. Daher bieten sich sowohl die Reduktion des betrachteten Systems auf kleinere Modellmoleküle an, die die gleichen primären Wechselwirkungen eingehen, als auch die Verwendung von Apparaturen wie dem Popcorn-Jet mit seiner Kombination aus heizbarer Probenkammer für die schwerflüchtige Substanz und Sättigersystem für die Anreicherung von Wasser im Trägergas.

Die mögliche Anwendungspalette für Biomolekül-Wasser-Koexpansionen ist enorm. Da aber speziell in den Aktivzentren von Enzymen einzelne Wassermoleküle von grundlegender Bedeutung für die Reaktionsmechanismen sein können ${ }^{7,8,195,197}$, sind Wechselwirkungen von Wasser mit den Seitenketten proteinogener Aminosäuren von besonderer Relevanz. Für die Untersuchung in der Gasphase bietet sich dabei der Einsatz der reinen Seitenkettenfunktionalitäten ohne die tatsächlichen Aminosäuregruppen an, da letztere zum einen innerhalb der Enzyme üblicherweise in Peptidketten eingebunden sind und zum anderen der unzersetzte Übergang der im festen Zustand zwitterionisch vorliegenden Aminosäuren ${ }^{198,199}$ in die Gasphase häufig nicht in ausreichendem Umfang $\mathrm{zu}$ realisieren ist. 
Während für einige Aminosäuren eine einzelne Geometrie der Wasserstoffbrückenbindung zwischen Wasser und der Seitenkette eindeutig bevorzugt ist, sind für andere verschiedene Anordnungen denkbar. Ein Beispiel hierfür ist die proteinogene Aminosäure Histidin $^{200}$, die eine Imidazolylgruppe in der Seitenkette aufweist. Imidazol (1,3-Diazol, $\mathrm{C}_{3} \mathrm{~N}_{2} \mathrm{H}_{4}$ ) ist ein aromatischer Heterocyclus, der sowohl eine $\mathrm{N}-\mathrm{H}$-Donor- als auch eine N-Akzeptorfunktionalität besitzt ${ }^{195}$ und somit wie Wasser beide Positionen innerhalb von Wasserstoffbrückenbindungen einnehmen kann. Außer in Histidin treten ImidazolUnterstrukturen auch beispielsweise in Purinen wie Adenin und Guanin als Teil der DNA auf, sind aber auch in Vitamin $B_{12}$ und dem biogenen Amin Histamin ${ }^{195,201}$ sowie zahlreichen pharmakologisch wirksamen Substanzen ${ }^{202}$ zu finden. Sie sind also in Organismen sehr präsent und aufgrund ihrer Donor- und Akzeptorgruppen sowie ihrer leichten Protonierbarkeit häufig essentiell für die jeweiligen Reaktionsabläufe. ${ }^{196,200}$ Gleichzeitig ist aber auch das reine Wasser-Imidazol-Bindungsmotiv für in vitro-Katalysen von praktischer Relevanz. ${ }^{203}$

Daher soll hier zunächst die Untersuchung der bevorzugten Wechselwirkung zwischen Wasser und Imidazol erfolgen, wobei der Fokus auf der Verteilung von Donor- und Akzeptorrolle im gemischten, wasserstoffbrückengebundenen Dimer liegt. Da Imidazol in biologischen Systemen jedoch nicht isoliert sondern als Imidazolylgruppe angebunden an weitere Funktionalitäten vorliegt (wie beispielsweise in Histidin), ergibt sich für diese Fälle zusätzlich die Frage des bevorzugten Tautomers. Als zweites Molekül soll daher 4beziehungsweise 5-Phenylimidazol ${ }^{204,205,206}$ untersucht werden, in dem zwar einerseits tautomere Formen vorliegen können, andererseits der Substituent selber aber keine zusätzlichen starken, gerichteten Wechselwirkungen mit Wasser eingehen sollte.

\subsection{Wasser und Imidazol}

Bei Imidazol $\left(\mathrm{C}_{3} \mathrm{~N}_{2} \mathrm{H}_{4}, i\right)$ handelt es sich um einen schwingungsspektroskopisch gut untersuchten Heterocyclus. Die stickstoffbasierten Donor- und Akzeptorfunktionalitäten im Molekül sind dabei weit genug voneinander entfernt, um sich, anders als beim Pyrazol $^{207}$, nicht gegenseitig zu beeinflussen. Es sind zwei verschiedene Strukturen des gemischten Dimers möglich: Entweder bindet eins der Wasserstoffatome des Wassermoleküls an das sich in der Ringebene befindende freie Elektronenpaar des einen Stickstoffs im Imidazol, oder eins der freien Elektronenpaare des Wasser-Sauerstoffs fungiert als Akzeptor für die N-H-Gruppe des anderen Imidazol-Stickstoffs. Eine Bindung an die ,aktivierte“ C2-H-Bindung des Imidazols ist hingegen sehr unwahrscheinlich. ${ }^{208,209}$ Somit gestaltet sich die Strukturaufklärung des gemischten Dimers mit Wasser problematischer als beim verwandten $\mathrm{Pyrrol}^{210,211}$, das nur eine N-H-Funktionalität aufweist, oder dem protonierten Imidazol ${ }^{8}\left(i \mathrm{H}^{+}\right)$mit zwei identischen $\mathrm{N}-\mathrm{H}$-Gruppen.

Bisherige Untersuchungen zur Struktur des energetisch bevorzugten gemischten Imidazol-Wasser-Dimers kamen zu keinem überzeugenden Resultat. Imidazol stellt in wässriger Lösung eine organische Base dar $\left(\mathrm{p} K_{\mathrm{a}}=14.2\right)^{200}$, so dass eine Dimerstruktur mit 


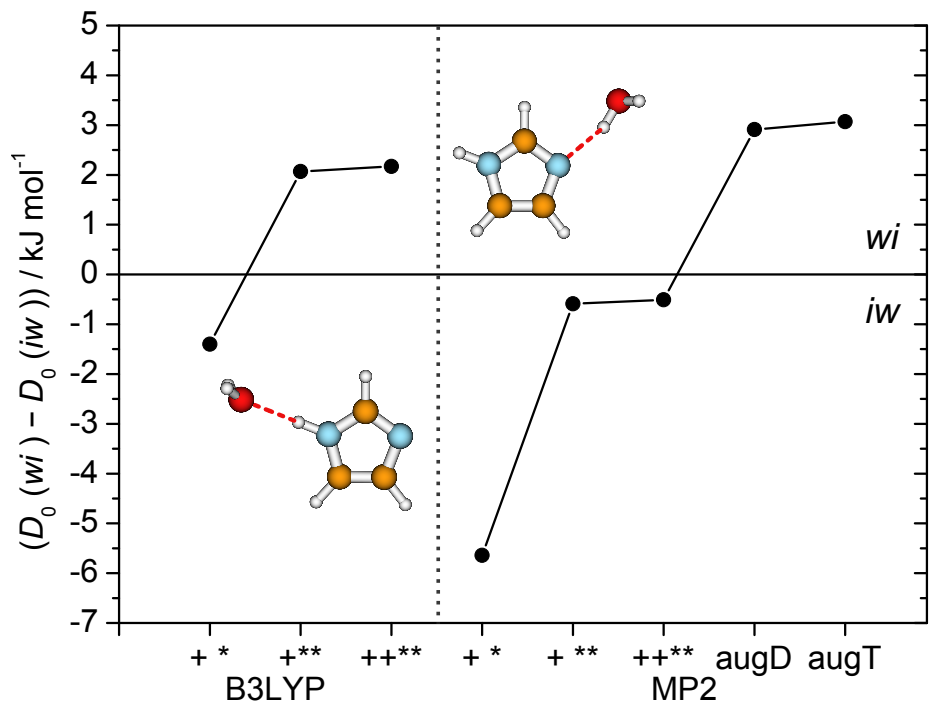

Abbildung 5.1: Basissatzabhängigkeit der Unterschiede in den nullpunktskorrigierten Dissoziationsenergien $D_{0}$ der beiden gemischten Imidazol-Wasser-Dimere in B3LYP- und MP-Rechnungen. ${ }^{117}$ Der Pople-Basissatz 6-311G wurde mit den angegebenen diffusen Funktionen $(+,++)$ und Polarisationsfunktionen $(*, * *)$ erweitert. Für die ab initioMethode MP2 wurden zusätzlich die Dunning-Basissätze aug-cc-pV(D,T)Z verwendet. Die zur Veranschaulichung der vorhergesagten Donor- und Akzeptorpräferenzen dargestellten Aggregatstrukturen wurden auf MP2/aug-cc-pVDZ-Niveau erhalten.

Akzeptor-Imidazol naheliegt. Allerdings muss das Verhalten kalter, isolierter 1:1-Dimere mit je nur einer Wasserstoffbrückenbindung nicht dem der makroskopischen wässrigen Lösung bei Zimmertemperatur entsprechen, da es sich bei der Lösung um ein dynamisches Gleichgewichtssystem mit einem typischerweise größeren Wasser/Imidazol-Verhältnis handelt und zusätzlich Effekte wie Kooperativität und intermolekulare Schwingungskopplung vorhanden sind. ${ }^{212}$ Quantenchemische Rechnungen ${ }^{117}$ führen in Abhängigkeit von Methode und Basissatz zu unterschiedlichen Resultaten. Werden Standard-Pople-Basissätze eingesetzt, ergibt sich bei B3LYP-Rechnungen ${ }^{121,213}$ überwiegend eine energetische Bevorzugung der $\mathrm{O}-\mathrm{H}$-Donor-Struktur wi, während MP2Rechnungen ${ }^{121,213,214}$ eher die N-H-Donor-Struktur $i w$ favorisieren. Insgesamt sind jedoch in fast allen Fällen die energetischen Unterschiede zwischen den beiden gemischten Imidazol-Wasser-Dimeren gering und betragen weniger als $5 \mathrm{~kJ} \mathrm{~mol}^{-1}$, häufig sogar weniger als $1 \mathrm{~kJ} \mathrm{~mol}^{-1}$. Die Abhängigkeit der Energiedifferenz der beiden Isomere vom verwendeten Basissatz ist in Abbildung 5.1 exemplarisch dargestellt. Die Nomenklatur der Heterodimere folgt dem Schema „Donorkürzel Akzeptorkürzel“, im Dimer wi ist also die O-H-Gruppe des Wassers $(w)$ der Donor und Imidazol $(i)$ fungiert als Akzeptor. Es zeigt sich, dass für MP2-Rechnungen beim Einsatz korrelationskonsistenter Dunning-Basissätze ${ }^{215}$ die Vorhersagen für die nullpunktskorrigierte Energiedifferenz schnell konvergieren und bereits der Einsatz des Triple-Zeta-Basissatzes aug-cc-pVTZ 
fast keine Veränderungen gegenüber dem Double-Zeta-Basissatz aug-cc-pVDZ bewirkt. Im Folgenden wird die Methode MP2/aug-cc-pVDZ für Betrachtungen gemischter Imidazol-Wasser- und Imidazol-Methanol-Dimere verwendet, da sie einen sinnvollen Kompromiss zwischen Rechenaufwand und Vorhersagegenauigkeit darstellt; mit ihr ergibt sich wi, also die O-H-Donor-Struktur, als energetisch begünstigtes Dimer. Beachtet werden muss allerdings, dass trotz guter Beschreibungen von Aggregatgeometrien und Dissoziationsenergien die Schwingungsvorhersagegenauigkeit von MP2/aug-cc-pVDZRechnungen für aromatische Systeme nur mäßig ist. ${ }^{216}$

Auch frühere experimentelle Arbeiten liefern kein klares Bild des bevorzugten Dimers. Statistische Betrachtungen wasserhaltiger Kristalle von Imidazolderivaten haben Wasser als den häufigeren Donor identifiziert ${ }^{209}$, die Umgebung im Einkristall stellt jedoch kein geeignetes Modell für isolierte Gasphasencluster oder Aktivzentren von Enzymen dar. Ergebnisse aus der Rydberg-Elektronen-Transfer-Spektroskopie weisen ebenfalls auf eine Donorrolle des Wassers hin, die Spektreninterpretation ist hier jedoch aufgrund von Unsicherheiten bezüglich berechneter und gemessener Bindungsenergie des Überschusselektrons sowie der Dipolmomente der flexiblen Dimere nicht eindeutig. ${ }^{217}$ FTIR-Spektren der Heterodimere in der Argon-Matrix ${ }^{213}$ zeigen das Vorliegen beider Isomere im Verhältnis wi : $i w=1: 1$ bis $2: 1$, allerdings unterliegt die Aggregatbildung in kryogenen Matrizen einer kinetischen Kontrolle und Einflüssen durch die Matrix-Umgebung. Somit liefern diese Spektren keine zuverlässigen Aussagen über thermodynamische Präferenzen. Weniger ausgeprägte Matrix-Effekte liegen innerhalb von Helium-Nanotröpfchen vor, in denen ebenfalls ein Isomerenverhältnis von etwa 1:1 nachgewiesen wurde ${ }^{218}$, die kinetische Kontrolle ist hier allerdings noch ausgeprägter. Auch wenn so ebenfalls keine Aussagen über energetische Präferenzen getroffen werden können, sind doch die Bandenpositionen der Infrarotspektren in Helium-Nanotröpfchen im Gegensatz zu denen aus der Matrixisolationsspektroskopie gut mit denen aus FTIRExperimenten an Überschallstrahlen vergleichbar.

Bevor die Popcorn-Jet-Spektren gemischter Imidazol-Solvens-Expansionen vorgestellt werden, soll hier zunächst eine Betrachtung der Spektren des reinen Imidazols stattfinden, um eine Übersicht über die auftretenden Banden und Aggregate zu erhalten. Abbildung 5.2 zeigt die Spektren der Expansionen von Imidazol in Helium bei einem Stagnationsdruck von $p_{0}=1.5$ bar und Substanztemperaturen von $110^{\circ} \mathrm{C}$ (a) und $130^{\circ} \mathrm{C}(\mathrm{b})$. Zusätzlich gezeigt ist das Spektrum der ersten Nachscans der Expansionen bei $\vartheta(i)=130{ }^{\circ} \mathrm{C}(\mathrm{c})$.

Das Probenspektrum a) bei geringerer Imidazolkonzentration zeigt zwei dominante Banden bei 3518 und $3214 \mathrm{~cm}^{-1}$, die der freien $\mathrm{N}-\mathrm{H}-$ Streckschwingung des ImidazolMonomers und der gebundenen des Imidazol-Dimers zugeordnet werden können. Zusätzlich sind schwächere Banden bei 3494, 3381 und $3322 \mathrm{~cm}^{-1}$ zu erkennen. Während die Bande bei $3494 \mathrm{~cm}^{-1}$ vermutlich der N-H-Streckschwingung von Akzeptormolekülen in Imidazol-Dimeren zugeschrieben werden kann, ist der Ursprung der anderen beiden Banden weniger eindeutig. Aufgrund ihrer Wellenzahlen zwischen der des ImidazolMonomers und der des Dimers kann gefolgert werden, dass sie ihren Ursprung in weni- 


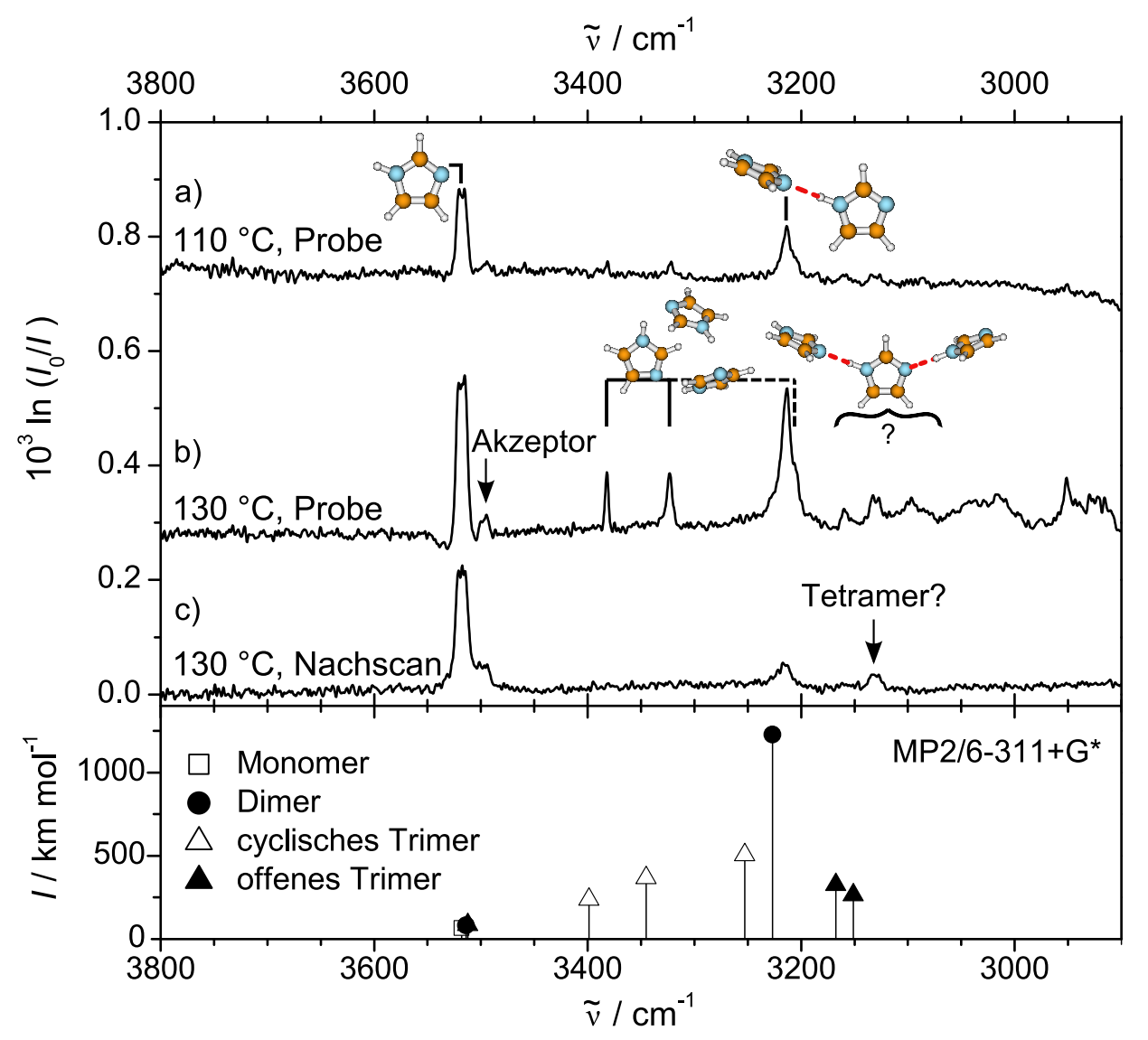

Abbildung 5.2: Popcorn-Jet-Expansionen von Imidazol in Helium $\left(p_{0}=1.5\right.$ bar) bei verschiedenen Substanztemperaturen und Vergleich mit berechneten Linienspektren verschiedener Imidazolaggregate. a) $\vartheta(i)=110^{\circ} \mathrm{C}$, Probescans. b) $\vartheta(i)=130{ }^{\circ} \mathrm{C}$, Probescans. c) $\vartheta(i)=130^{\circ} \mathrm{C}, 1$. Nachscans.

Berechnete Spektren wurden auf MP2/6-311+G*-Niveau nach Verschiebung der Spektren um $-160 \mathrm{~cm}^{-1}$ zur Übereinstimmung der experimentellen und theoretischen $\mathrm{N}-\mathrm{H}-$ Streckschwingung des Imidazol-Monomers erhalten. 
ger starken Wasserstoffbrückenbindungen haben, als sie im Dimer auftreten. In Frage kommen hierfür entweder Mischaggregate mit Verunreinigungen wie Wasser oder aber größere reine Imidazol-Aggregate, auch wenn die bisherigen Betrachtungen von Methanol und Wasser selbst für die gespannten cyclischen Trimere Wellenzahlen unterhalb der des Dimers ergeben haben. Bei einer Erhöhung der Probenkammertemperatur auf $130^{\circ} \mathrm{C}$ und entsprechend höherer Imidazol-Konzentration in der Expansion (Spektrum 5.2 b) zeigt sich ein deutlicher Intensitätsanstieg aller drei zuvor schwachen Banden, so dass Verunreinigungen als Ursache praktisch auszuschließen sind. Eine Zuordnung der Banden bei 3381 und $3322 \mathrm{~cm}^{-1}$ zu Aggregaten aus mindestens drei Imidazolmolekülen ist hingegen wahrscheinlich, während der Intensitätsanstieg der Akzeptorbande bei $3494 \mathrm{~cm}^{-1}$ auf die gestiegene Dimerkonzentration zurückgeführt werden kann.

Rechnungen auf B3LYP-D/aug-cc-pVDZ-Niveau haben für die Imidazol-Trimere zwei verschiedene stabile Strukturen vorhergesagt. ${ }^{201}$ Hierbei handelt es sich um eine stark gespannte, asymmetrische Ringstruktur und eine um etwa $21 \mathrm{~kJ} \mathrm{~mol}^{-1}$ weniger stabile, kettenförmige Struktur. ${ }^{201}$ Ähnliche Ergebnisse liefern auch MP2/6-311+G*-Rechnungen. Die so erhaltenen Geometrien der Imidazol-Aggregate und nullpunktskorrigierte Dissoziationsenergien $D_{0}$ sind in der oberen Hälfte von Abbildung 5.3 dargestellt. Die zugehörigen Strichspektren der harmonischen Frequenzrechnungen befinden sich zum Vergleich mit den experimentellen Spektren unten in Abbildung 5.2. Die theoretischen Wellenzahlen wurden dabei um $-160 \mathrm{~cm}^{-1}$ verschoben um eine Übereinstimmung der berechneten mit der experimentellen $\mathrm{N}-\mathrm{H}$-Streckschwingungsbande des Imidazol-Monomers zu erreichen. Für das Dimer ergibt sich so eine Abweichung zwischen theoretischer und gemessener Donorbandenposition von $13 \mathrm{~cm}^{-1}$, die Übereinstimmung ist also relativ gut. Auch für die zwei Banden bei 3381 und $3322 \mathrm{~cm}^{-1}$ ergibt sich eine Differenz zu den vorhergesagten Banden des cyclischen Trimers von je nur etwa $20 \mathrm{~cm}^{-1}$, so dass eine entsprechende Zuordnung sinnvoll ist. Die am weitesten rotverschobene der drei Ringtrimer-Streckschwingungsbanden ist vermutlich von der Dimerbande überlagert und somit nicht eindeutig zu identifizieren. Die hohen N-H-Streckschwingungswellenzahlen und starke Bandenaufspaltung des cyclischen Trimers sind vermutlich auf die geringe Symmetrie des Aggregats, die hohe Ringspannung und die aufgrund der Separierung von Donor- und Akzeptorfunktionalität geringere Kooperativität im Vergleich zu beispielsweise Wasser zurückzuführen.

Zusätzlich werden bei der höheren Substanztemperatur in Spektrum 5.2 b noch weitere Banden unterhalb von $3200 \mathrm{~cm}^{-1}$ sichtbar. Hierbei kann es sich sowohl um die relativ schwachen $\mathrm{C}-\mathrm{H}-\mathrm{Streckschwingungsbanden} \mathrm{des} \mathrm{Monomers} \mathrm{und} \mathrm{der} \mathrm{Aggregate} \mathrm{als}$ auch um N-H-Schwingungen größerer Aggregate und möglicherweise auch des offenkettigen Trimers handeln, wobei dies aufgrund der großen Energiedifferenz zum cyclischen Trimer (s. Abb. 5.3) eher unwahrscheinlich ist. Weitere Informationen liefert das in Abbildung 5.2 c gezeigte Spektrum der Nachscans, die im Mittel etwa 200 ms nach Beendigung der Gaspulse gemessen werden. Die so aufgenommene Gasphase enthält im Wesentlichen Monomere sowie stabile Aggregate. Neben den Banden des Imidazol-Monomers und des Dimers ist im Spektrum nur eine zusätzliche Bande bei etwa $3130 \mathrm{~cm}^{-1}$ 


\begin{tabular}{lcccc}
\hline \multirow{2}{*}{ Aggregat } & & \multicolumn{2}{c}{$\tilde{\nu} / \mathrm{cm}^{-1}$} & \\
& Popcorn-Jet & $\mathbf{H e}^{218}$ & He (korr.) & \\
& Ard & Ar-Matrix $^{213}$ \\
\hline \hline$i$ & 3518 & 3517.9 & - & 3500 \\
$i i^{\mathrm{D}}$ & 3214 & 3200.1 & $\approx 3214$ & - \\
$i i^{\mathrm{A}}$ & 3494 & 3515.8 & - & - \\
$i w$ & - & 3411.8 & $\approx 3418$ & 3373 \\
$w i$ & 3458 & 3447.9 & $\approx 3458$ & 3394 \\
\hline
\end{tabular}

Tabelle 5.1: Wellenzahlen der intensiven experimentellen Streckschwingungsbanden des Imidazol-Monomers $(i)$, -Dimers $(i i)$ sowie der gemischten Dimere von Imidazol mit Wasser (iw: N-H-Donor, wi: O-H-Donor). Vergleich der Popcorn-Jet-Ergebnisse (siehe auch Referenz 121) mit denen aus Helium-Nanotröpfchen-Experimenten ${ }^{218}$ (ohne und mit Einbeziehung der bekannten Verschiebungen ${ }^{219}$ gegenüber Gasphasenexperimenten) sowie denen aus Argon-Matrizen ${ }^{213}$. Superskript D: Donorbande, A: Akzeptorbande.

zu erkennen. Diese könnte auf das cyclische, sehr stabile Tetramer zurückzuführen sein, da das Tetramer mit einer berechneten Dissoziationsenergie pro Wasserstoffbrückenbindung von $47 \mathrm{~kJ} \mathrm{~mol}^{-1}$ als einziges kleines Aggregat stärker als das Dimer gebunden ist. ${ }^{201}$ Ohne die Ergänzung durch (beispielsweise) Raman-spektroskopische Messungen kann jedoch eine alternative Bandenzuordnung wie etwa zum offenkettigen ImidazolTrimer nicht ausgeschlossen werden. Das Wegfallen der übrigen Banden bestätigt ihren Ursprung in Schwingungen schwächer gebundener Aggregate. Zur Vermeidung unnötig hoher Komplexität der erhaltenen Spektren wurde für die Koexpansionen mit Solvensmolekülen eine Imidazol-Temperatur von $110^{\circ} \mathrm{C}$ gewählt.

Nachdem sich der Einsatz von Methanol als einfacheres Modell für Wasser bereits in Kapitel 4.1 bewährt hat, sollen auch hier zunächst Koexpansionen von Imidazol und Methanol untersucht werden. Neben seiner Modellfunktion besitzt dieses System zusätzlich auch eine praktische Relevanz für Membranen in Direkt-Methanol-Brennstoffzellen, in denen zur Verbesserung der Protonenleitfähigkeit Imidazolylsubstituenten eingesetzt werden. ${ }^{220,221}$

Abbildung 5.4 zeigt sowohl die Spektren der Einzelkomponentenexpansionen von Imidazol (a) und Methanol (b) in Helium als auch das Koexpansionsspektrum beider Substanzen unter ansonsten konstanten Bedingungen (c) und das Koexpansionsspektrum nach skalierter Subtraktion der Einzelspektren (d). Zum Vergleich sind die berechneten (MP2/aug-cc-pVDZ) N-H- und O-H-Streckschwingungsspektren der Heterodimere von Imidazol $(i)$ und Methanol $(m)$, im und $m i$, nach einer Verschiebung der Wellenzahlen um $-144 \mathrm{~cm}^{-1}$ zur Übereinstimmung der berechneten und experimentellen N-H-Streckschwingungsbande des Imidazol-Monomers gezeigt. Während für die Bestimmung verlässlicher Absolutwerte der Wellenzahlen und Dissoziationsenergien anharmonische Rechnungen unter Berücksichtigung des Basissatzüberlagerungsfehlers (basis set superposition error, BSSE) notwendig wären, sollen an dieser Stelle vor allem 


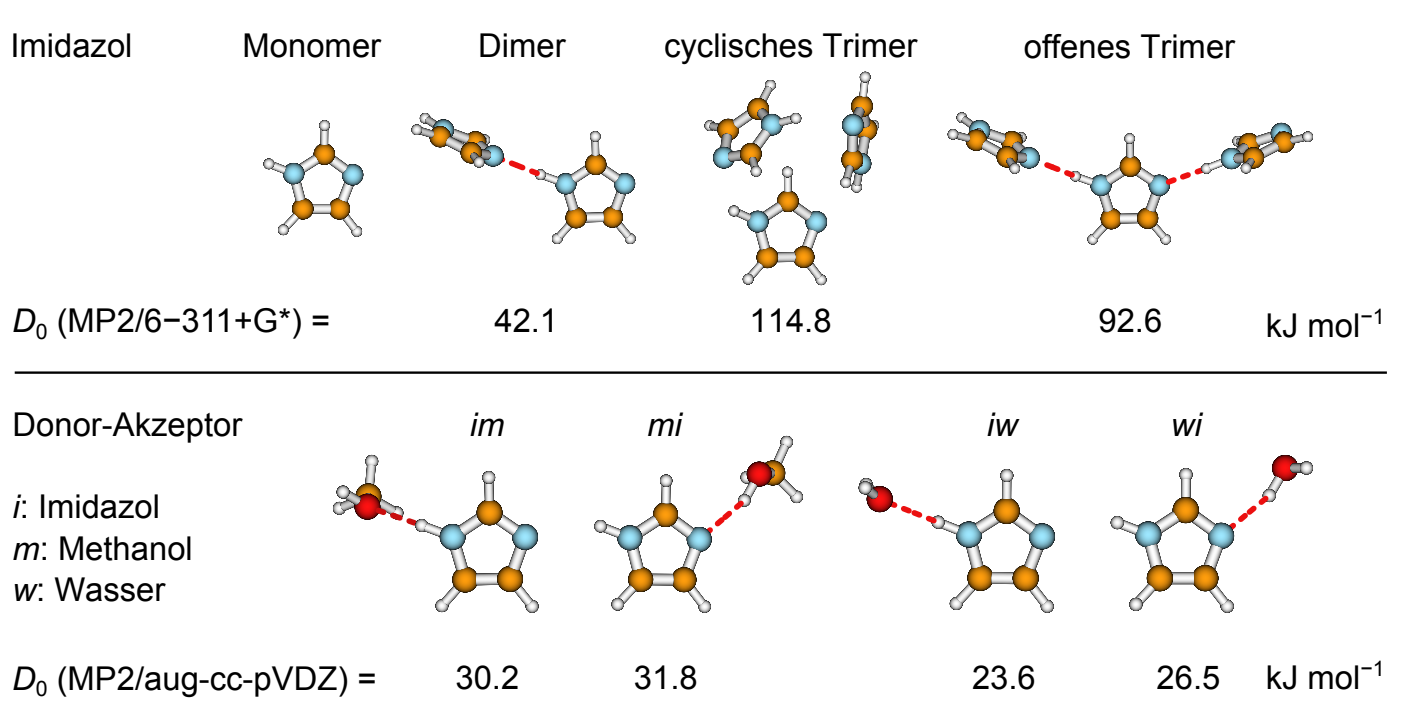

Abbildung 5.3: Berechnete Strukturen und nullpunktskorrigierte Dissoziationsenergien für kleine Imidazolaggregate (MP2/6-311+ $\mathrm{G}^{*}$, oben) und gemischte Imidazol-Methanolund Imidazol-Wasser-Dimere (MP2/aug-cc-pVDZ, unten). Weitere Informationen zu Energien, Wellenzahlen und Intensitäten befinden sich in Abschnitt A.4.

qualitative Aussagen getroffen werden, so dass harmonische Rechnungen ohne BSSEKorrekturen hinreichend sind.

Die Konzentrationen der Einzelsubstanzen in den Überschallexpansionen wurden gering gewählt, um signifikante Anteile von Trimeren und größeren Aggregaten bei der Koexpansion zu vermeiden. Für Imidazol wurde daher die geringere Substanztemperatur von $110^{\circ} \mathrm{C}$ aus Spektrum 5.2 a gewählt, so dass vor allem Monomer- und Dimerbanden erkennbar sind. Bei Methanol wurde durch das apparativ erreichbare Temperaturminimum des Sättigers von $-28^{\circ} \mathrm{C}$ und zusätzliche Verdünnung über eine weitere Helium-Zumischung die Konzentration so niedrig gehalten, dass in Spektrum 5.4 b praktisch nur die O-H-Streckschwingungsbande des Methanol-Monomers bei $3686 \mathrm{~cm}^{-1}$ sichtbar ist. Das Koexpansionsspektrum c) von Imidazol und Methanol zeigt neben den Reinsubstanzbanden bereits deutlich eine zusätzliche Bande bei $3439 \mathrm{~cm}^{-1}$ und eine Verbreiterung der schwachen Imidazol-Trimerbande bei $3381 \mathrm{~cm}^{-1}$, die nach Subtraktion der Homoaggregatbanden in Spektrum d) als separate Bande bei $3377 \mathrm{~cm}^{-1}$ erkennbar wird. Beide Banden sind somit auf gemischte Imidazol-MethanolAggregate zurückzuführen, wobei aufgrund der geringen Konzentrationen und fehlenden weiteren Banden (abgesehen von einer Restbande des Imidazol-Dimers) eine Zuordnung zu gemischten Dimeren naheliegend ist. Weitere schwache Banden unterhalb von $3100 \mathrm{~cm}^{-1}$ sind nicht eindeutig identifizierbar und teilweise durch Restintensitäten der Methanol-C-H-Streckschwingungen verursacht, sie sollen an dieser Stelle nicht eingehend betrachtet werden.

Ein Vergleich mit den berechneten Strichspektren liefert insbesondere für die stär- 


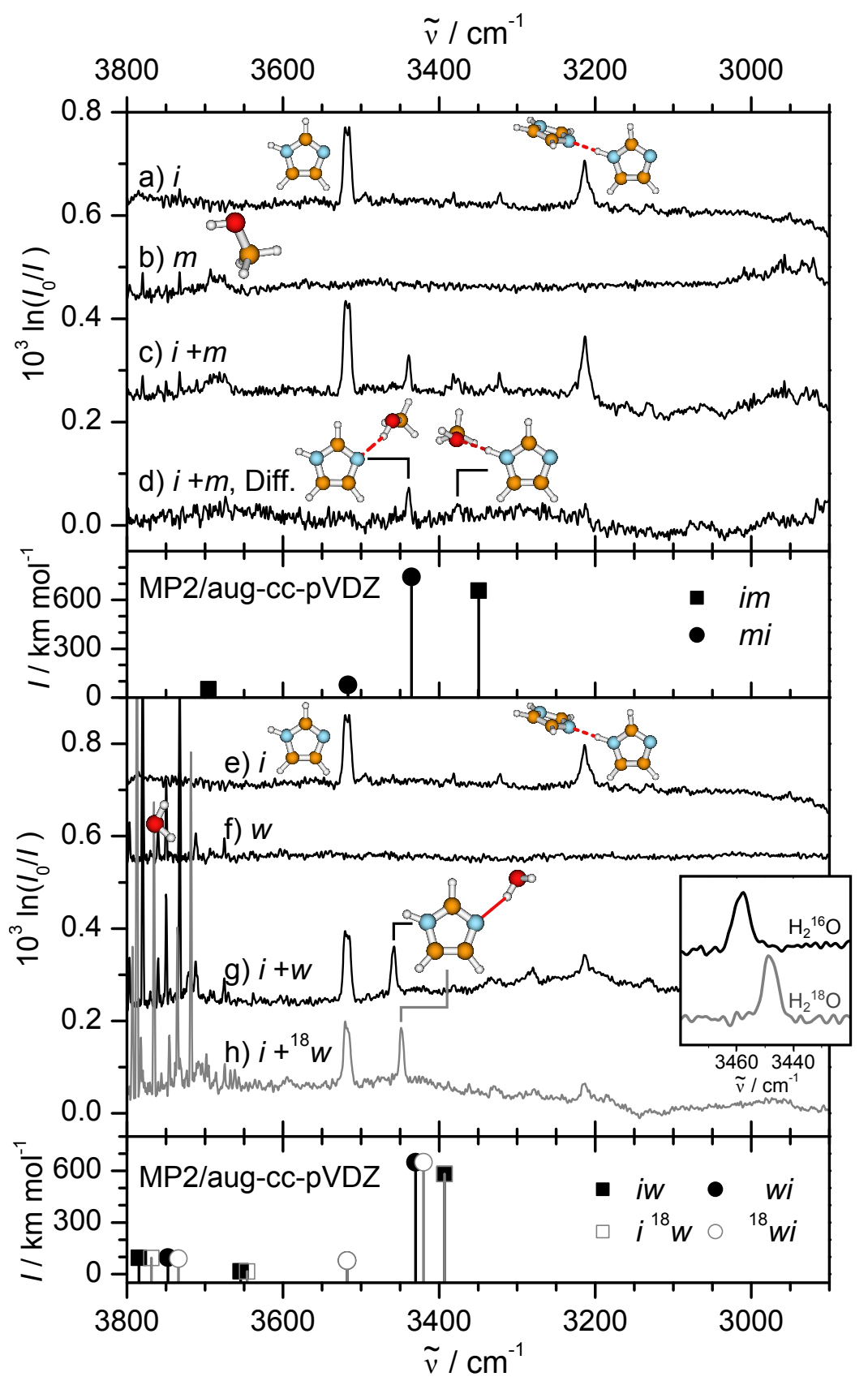

Abbildung 5.4: Einzelsubstanz- und Koexpansionsspektren der Popcorn-Jet-Expansionen von Imidazol und Methanol bzw. Imidazol und Wasser (Trägergas Helium, $p_{0}=1.5$ bar) und Vergleich mit berechneten Linienspektren (MP2/aug-cc-pVDZ, $-144 \mathrm{~cm}^{-1}$ ). a) Imidazol. b) Methanol. c) Koexpansion von Imidazol und Methanol. d) Entspricht c) nach skalierter Subtraktion von a) und b). e) Imidazol. f) Wasser. g) Koexpansion von Imidazol und Wasser. h) Koexpansion von Imidazol und ${ }^{18}$ O-isotopenmarkiertem Wasser. Die Ausschnittsvergrößerung zeigt die Streckschwingungsbanden gemischter Imidazol-Wasser-Dimere aus Spektren g) und h). 
kere Heteroaggregatbande bei $3439 \mathrm{~cm}^{-1}$ eine sehr gute Übereinstimmung mit der vorhergesagten Donor-O-H-Streckschwingungsbande des mi-Dimers. Jedoch ist auch die Differenz zwischen der zweiten Bande bei $3377 \mathrm{~cm}^{-1}$ und der theoretischen im-N-HStreckschwingungsbande mit $28 \mathrm{~cm}^{-1}$ noch im akzeptablen Bereich, zumal auch die relative Lage der beiden gemischten Dimerbanden zueinander gut wiedergegeben wird. Ein Vergleich der nullpunktskorrigierten Dissoziationsenergien der Heterodimere in Abbildung 5.3 zeigt eine mit weniger als $2 \mathrm{~kJ} \mathrm{~mol}^{-1}$ geringfügig höhere Stabilität der $m i$ gegenüber der $\mathrm{im}$-Geometrie, die sich gut mit den beobachteten relativen Bandenintensitäten im Differenzspektrum 5.4 d deckt. Auf diese Weise ergibt sich eine schlüssige Zuordnung für beide beobachtete Mischaggregatbanden, die zur nun folgenden Interpretation der Imidazol-Wasser-Koexpansionsspektren beitragen kann. Da die berechnete Energiedifferenz für die analogen gemischten Dimere wi und $i w$ mit etwa $3 \mathrm{~kJ} \mathrm{~mol}^{-1}$ fast doppelt so groß ist wie für Methanol, sollte sich dies auch in den Mischclusterbandenintensitäten widerspiegeln.

Abbildung 5.4 e zeigt zur besseren Vergleichbarkeit noch einmal das Spektrum der Expansion von Imidazol $\left(110^{\circ} \mathrm{C}\right)$ in Helium aus Abbildung 5.2 a. Die Konzentration von Wasser wurde - analog zum Methanol - durch Wahl einer Temperatur von $2{ }^{\circ} \mathrm{C}$ so eingestellt, dass im Spektrum $5.4 \mathrm{f}$ bei $3600 \mathrm{~cm}^{-1}$ keine Bande des Wasser-Dimers erkennbar ist. Für die Identifikation der Schwingungen von Imidazol-Wasser-Aggregaten stehen Daten aus Helium-Nanotröpfchen-Experimenten zur Verfügung. ${ }^{218}$ Bei diesen kann die Zuordnung zuverlässig durch die Polarisationsabhängigkeit der Bandenintensität einer betrachteten Schwingungsmode und dem daraus gefolgerten Winkel zwischen der Richtung des Übergangsdipolmoments der betrachteten Mode und der des permanenten Dipolmoments des Aggregats erfolgen. ${ }^{219}$ Die Bandenpositionen der Monomere zeigen üblicherweise keine substantiellen Unterschiede zu denen aus Überschallexpansionen, während bei den Streckschwingungen wasserstoffbrückengebundener X-H-Oszillatoren ein bekannter linearer Zusammenhang zwischen Bandenverschiebung relativ zum Gasphasenspektrum und Rotverschiebung relativ zur freien X-H-Monomerschwingung besteht: ${ }^{219}$

$$
\tilde{\nu}_{\text {gas }}-\tilde{\nu}_{\mathrm{He}} \approx 0.04 \cdot \Delta \tilde{\nu}_{\text {rot }}+1.6 \mathrm{~cm}^{-1}
$$

Die erhaltenen Bandenpositionen können daher für den Vergleich mit den Messungen im Überschallstrahl korrigiert werden. So erhaltene unkorrigierte und korrigierte Werte für das Imidazol-Monomer und die Dimere $i i$, $i w$ und wi sind in Tabelle 5.1 aufgeführt. Dabei wurde für den Wert der Rotverschiebung die Wellenzahldifferenz zwischen der Clusterbande und dem jeweiligen Donor-Monomer in Helium-Nanotröpfchen verwendet, wobei für Imidazol die Wellenzahl der N-H-Streckschwingung von $3518 \mathrm{~cm}^{-1}$ und für Wasser die der symmetrischen Streckschwingung ${ }^{163}$ bei $3657 \mathrm{~cm}^{-1}$ eingesetzt wurde.

Das erhaltene Koexpansionsspektrum 5.4 g von Imidazol und Wasser zeigt (im Unterschied zum Koexpansionsspektrum c mit Methanol) nur eine zusätzliche Bande im aus den Helium-Nanotröpfchen bekannten relevanten Spektralbereich zwischen 3400 und $3500 \mathrm{~cm}^{-1}$, wie es aufgrund der höheren Energiedifferenz zwischen den beiden He- 
terodimerisomeren auch plausibel ist. Die Bandenposition entspricht mit $3458 \mathrm{~cm}^{-1}$ exakt dem korrigierten Wert aus den He-Tröpfchen-Messungen für das wi-Dimer mit Wasser als Wasserstoffbrückendonor. Die entsprechenden Werte können Tabelle 5.1 entnommen werden. Ebenso stimmt die Bandenposition mit einer Abweichung von nur etwa $20 \mathrm{~cm}^{-1}$ recht gut mit der des analogen Methanol-Aggregats $m i$ überein. Auch ein Vergleich mit den theoretischen Spektren auf MP2/aug-cc-pVDZ-Niveau in Abbildung 5.4, deren Wellenzahlen analog zu denen im Methanol-Fall verschoben wurden, zeigt eine Abweichung von nur $8 \mathrm{~cm}^{-1}$ zwischen der berechneten wi-Bande und der experimentellen bei $3458 \mathrm{~cm}^{-1}$. Unterstützt wird diese Zuordnung zudem noch durch die energetische Begünstigung gegenüber dem $i w$-Isomer. Dieses müsste entsprechend der korrigierten Werte für die Infrarotspektren der Helium-Nanotröpfchen-Messungen ${ }^{218}$ in Tabelle 5.1 eine $\mathrm{N}-\mathrm{H}$-Streckschwingungswellenzahl von etwa $3418 \mathrm{~cm}^{-1}$ aufweisen, das Koexpansionsspektrum 5.4 g lässt hier jedoch keine Bande erkennen.

Da die theoretischen Spektren für die Imidazol-Wasser-Heterodimere in Abbildung 5.4 eine Wellenzahldifferenz zwischen den beiden Strukturen $i w$ und $w i$ von weniger als $40 \mathrm{~cm}^{-1}$ voraussagen, ist eine Überlappung der Banden beider Isomere im experimentellen Spektrum denkbar. Dies würde allerdings selbst bei der gemessenen Bandenbreite der Mischaggregatbande bei $3458 \mathrm{~cm}^{-1}$ in Spektrum g) von $7 \mathrm{~cm}^{-1}$ noch einer durch die Helium-Nanotröpfchen-Umgebung verursachten Bandenverschiebung von über $40 \mathrm{~cm}^{-1}$ entsprechen, die in dieser Größenordnung sehr unwahrscheinlich ist. ${ }^{219}$ Dennoch soll an dieser Stelle ein experimenteller Nachweis durch Isotopenmarkierung erfolgen. Der Einsatz einer deuterierten Substanz ist hierbei nicht möglich, da sich die Komponenten einer Koexpansion beim Popcorn-Jet bereits vor der Expansion vermischen und so ein Isotopenaustausch erfolgen würde. Da der Muesli-Jet über keine Vorrichtung zur Aufheizung von Substanzen verfügt und der Dampfdruck von Imidazol bei Zimmertemperatur gering ist, wurde ${ }^{18} \mathrm{O}$-markiertes Wasser $\left({ }^{18} w\right)$ für die Popcorn-JetKoexpansionen verwendet. Auch hiermit erfolgt eine Verschiebung wasserstoffbrückengebundener $\mathrm{X}-\mathrm{H}$-Dimerschwingungen durch die Isotopenmarkierung nur dann, wenn die markierte Komponente (hier Wasser) als Donor fungiert. Die Wellenzahländerung ist jedoch im Vergleich zur Deuterierung klein, da der Einfluss auf die reduzierte Masse des Oszillators geringer ist.

Das Spektrum der Koexpansion von Imidazol und $\mathrm{H}_{2}{ }^{18} \mathrm{O}$ in Abbildung $5.4 \mathrm{~h}$ zeigt gegenüber dem mit $\mathrm{H}_{2}{ }^{16} \mathrm{O}$ in Abbildung $5.4 \mathrm{~g}$ für die Bande des gemischten Aggregats eine Rotverschiebung um $10 \mathrm{~cm}^{-1}$ ohne Restintensität bei $3458 \mathrm{~cm}^{-1}$, die gezeigte Ausschnittsvergrößerung bestätigt die Beobachtung. Diese Rotverschiebung entspricht auch der durch die MP2/aug-cc-pVDZ-Rechnungen für das wi-Dimer ermittelten, während für das $i w$-Isomer keine Bandenverschiebung zu erwarten ist.

Offenbar nimmt im System Imidazol-Wasser das Wasser ausschließlich die Donorrolle ein, wobei die Ausprägung dieser Präferenz bei einer berechneten Energiedifferenz von nur $3 \mathrm{~kJ} \mathrm{~mol}^{-1}$ gegenüber dem $i w$-Dimer einerseits auf die geringen Temperaturen in Überschallexpansionen zurückgeführt werden kann. Andererseits wurden in HeliumNanotröpfchen ${ }^{218}$ und in der Argon-Matrix ${ }^{213}$ beide Heterodimere gefunden, so dass 
die Bevorzugung im Jet nicht allein der Temperatur geschuldet sein kann, sondern auch dem geringeren Ausmaß an kinetischer Kontrolle bei der Aggregatbildung im Jet. Dabei ist zwar davon auszugehen, dass sich auch in der Überschallexpansion durch geeignete Stoßgeometrien zunächst beide Dimere bilden, die N-H-Donor-Aggregate iw aufgrund der hohen Anzahl an Stößen bei geringen Düsenabständen jedoch praktisch vollständig isomerisieren. Ein möglicher Mechanismus hierfür könnte eine intermolekulare Variante der lösungsmittelunterstützten Konformationsisomerisierung ${ }^{222}$ sein. Lagert sich an ein $i w$-Dimer ein weiteres Wassermolekül als Donor an das freie Elektronenpaar des zweiten Stickstoffs an, so könnte die frei werdende Bindungsenergie durch Umverteilung innerhalb des Aggregats zur Dissoziation des schwächer gebundenen Wassermoleküls führen ${ }^{223}$ und so eine Isomerisierung befördern. Dieser Prozess ist in der kollisionsreichen Zone einer Überschallexpansion dicht hinter dem Düsenausgang sehr viel wahrscheinlicher als in kryogenen Matrizen ${ }^{213}$ oder Lösungsmitteln ${ }^{218}$, so dass auf diese Weise die Diskrepanz zu den dort erhaltenen Isomerenhäufigkeiten erklärt werden kann.

Nachdem die Bildungspräferenz bei isolierten wasserstoffbrückengebundenen Dimeren zugunsten der Struktur mit Wasser als Donor und Imidazol als Akzeptor nachgewiesen wurde, lohnt trotz der bereits angesprochenen grundlegenden Unterschiede ein Vergleich mit dem makroskopischen Fall von Imidazol in wässriger Lösung. Die $\mathrm{p} K_{\mathrm{a}}$ Werte von Imidazol (14.2) und protoniertem Imidazol (7.1) sind bekannt: ${ }^{200}$

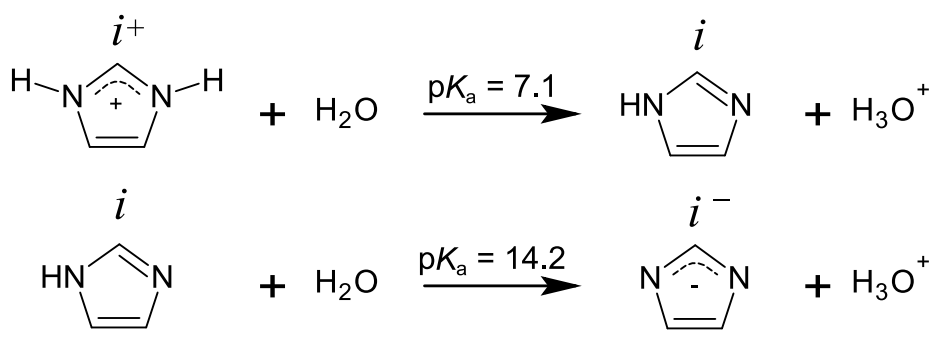

Für die Konzentrationen $c$ in neutraler wässriger Lösung folgt:

$$
\begin{aligned}
\frac{c(i) \cdot c\left(\mathrm{H}_{3} \mathrm{O}^{+}\right)}{c\left(i^{+}\right)} & =10^{-7.1} \frac{\mathrm{mol}}{\mathrm{L}} \\
\frac{c\left(i^{-}\right) \cdot c\left(\mathrm{H}_{3} \mathrm{O}^{+}\right)}{c(i)} & =10^{-14.2} \frac{\mathrm{mol}}{\mathrm{L}} \\
c\left(\mathrm{H}_{3} \mathrm{O}^{+}\right) & =10^{-7} \frac{\mathrm{mol}}{\mathrm{L}}
\end{aligned}
$$

Unter Annahme eines Gleichgewichtszustands bei Zimmertemperatur $T_{300}$ ergibt sich 
so die Differenz der freien Standard-Enthalpie $\Delta G^{\ominus}$ zwischen protoniertem und deprotoniertem Imidazol als:

$$
\begin{aligned}
\Delta G^{\ominus} & =-\ln \left(\frac{c\left(i^{+}\right)}{c\left(i^{-}\right)}\right) \cdot R \cdot T_{300} \\
& =-\ln \left(\frac{c(i) \cdot c\left(\mathrm{H}_{3} \mathrm{O}^{+}\right)}{10^{-7.1 \frac{\mathrm{mol}}{\mathrm{L}}}} \cdot \frac{c\left(\mathrm{H}_{3} \mathrm{O}^{+}\right)}{c(i) \cdot 10^{-14.2} \frac{\mathrm{mol}}{\mathrm{L}}}\right) \cdot 8.31447 \frac{\mathrm{J}}{\mathrm{mol} \mathrm{K}} \cdot 300 \mathrm{~K} \\
& \approx-42 \frac{\mathrm{kJ}}{\mathrm{mol}}
\end{aligned}
$$

Das System Imidazol-Wasser ist somit ein Beispiel dafür, wie sich ein ausgeprägtes makroskopisches Verhalten aus einem geringfügigen Energieunterschied auf der Ebene einzelner Wasserstoffbrückenbindungen entwickeln kann. Gleichzeitig stellt es ein Modell für Histidin-Wasser-Wechselwirkungen innerhalb der aktiven Zentren von Enzymen wie beispielsweise den Serinproteasen ${ }^{7}$ dar, wo es innerhalb einer katalytischen Triade den Protonentransfer im Peptidspaltungsprozess ermöglicht.

\subsection{Wasser und 4-/5-Phenylimidazol}

Im Gegensatz zum Modellsystem Imidazol kann die proteinogene Aminosäure Histidin in zwei verschiedenen Tautomeren vorliegen, da die Anbindung der Imidazolylgruppe sowohl an der 4-Position (neben dem Akzeptor-Stickstoff) oder an der 5-Position (neben der N-H-Gruppe) erfolgen kann. Durch Protonentransfer sind beide Formen ineinander überführbar, so dass sich anstelle der bislang betrachteten zwei gemischten Dimerstrukturen für Imidazol und Wasser mindestens vier Möglichkeiten für Histidin und Wasser ergeben. Ramanspektroskopische Messungen haben eine Bevorzugung der Anbindung am 4-C-Kohlenstoff mit einer Energiedifferenz von etwa $4 \mathrm{~kJ} \mathrm{~mol}^{-1}$ relativ zur 5-Position ergeben. ${ }^{224}$ Da Histidin selbst aufgrund der vielfältigen Wechselwirkungsmöglichkeiten zwischen Wasser und der Aminosäuregruppe ${ }^{199}$ sowie des geringen Dampfdrucks nicht für Koexpansionen eingesetzt werden soll, ist ein entsprechendes Modellsystem mit vergleichbaren tautomeren Formen notwendig.

Hierfür bietet sich 4-/5-Phenylimidazol $(4 p, 5 p)$ an, da es strukturell dem Histidin stark ähnelt und die Anbindung der Phenylgruppe an den 4-C-Kohlenstoff mit einer (rechnerischen) energetischen Präferenz ${ }^{204,225,226}$ von $3-5 \mathrm{~kJ} \mathrm{~mol}^{-1}$ bevorzugt ist. Zudem sind die möglichen gerichteten Wechselwirkungen zwischen der Phenylgruppe und Wasser im Wesentlichen auf schwache $\mathrm{C}-\mathrm{H} \cdots \mathrm{O}$ - und $\mathrm{O}-\mathrm{H} \cdots \pi$-Wasserstoffbrückenbindungen beschränkt, die gegenüber starken $\mathrm{O}-\mathrm{H} \cdots \mathrm{N}-$ und $\mathrm{N}-\mathrm{H} \cdots \mathrm{O}$-Bindungen von geringerer Relevanz sein sollten. Zusätzlich besitzt Phenylimidazol einen für Untersuchungen mit dem Popcorn-Jet ausreichenden Dampfdruck, so dass auch die praktische Umsetzbarkeit gegeben ist. Bemerkenswert ist außerdem die in RIDIR- („Resonant Ion Dip InfraRed-“) Spektren der Überschallexpansion von Phenylimidazol in Helium 


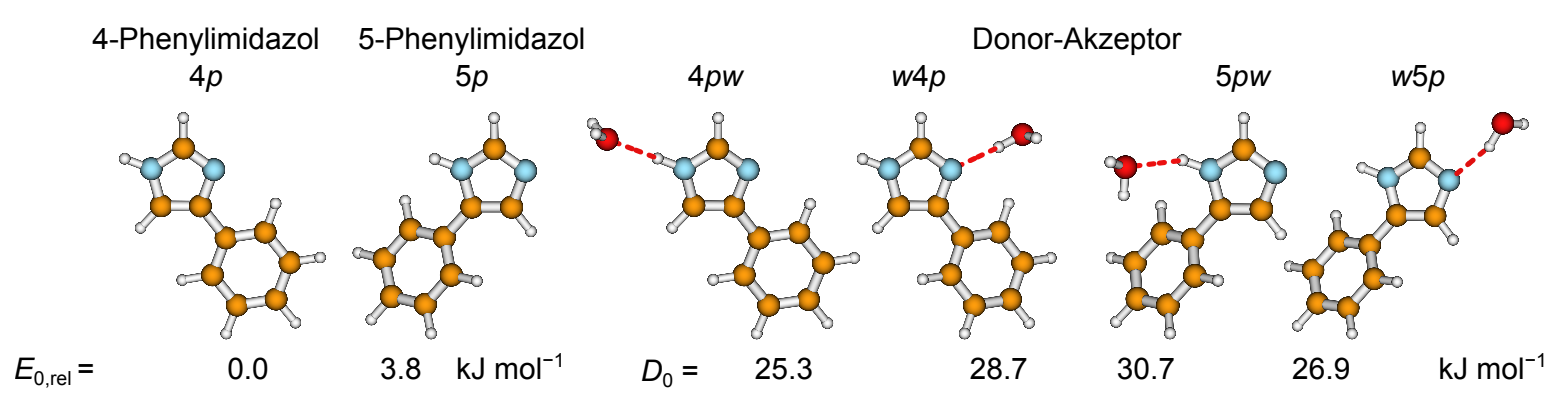

Abbildung 5.5: Berechnete (MP2/aug-cc-pVDZ) Strukturen und nullpunktskorrigierte relative $\left(E_{0, \text { rel }}\right)$ und Dissoziationsenergien $\left(D_{0}\right)$ der Tautomere 4- und 5-Phenylimidazol $(4 p, 5 p)$ sowie ihrer Heterodimere mit Wasser $(w)$. Aggregatnomenklatur: „Donorkürzel Akzeptorkürzel". Die Dissoziationsenergien sind relativ zu den jeweiligen Monomeren angegeben. Weitere Informationen zu Energien, Wellenzahlen und Intensitäten befinden sich im Anhang A.4.

gefundene ausschließliche Bildung von $4 p w$-Heterodimeren ${ }^{204,206}$, in denen die $\mathrm{N}-\mathrm{H}$ Gruppe des 4-Phenylimidazols den Wasserstoffbrückendonor und Wasser den Akzeptor darstellt. Diese Ergebnisse widersprechen der in Abschnitt 5.1 belegten Donorrolle von Wasser im gemischten Dimer mit Imidazol, so dass die Untersuchung dieser Diskrepanz zusätzlich von Interesse ist.

Während Phenylimidazol im Kristall ausschließlich in der $4 p$-Form vorliegt, können in Lösung abhängig vom Lösungsmittel etwa 10 bis $20 \%$ des $5 p$-Isomers nachgewiesen werden. ${ }^{227}$ In der Gasphase ist der Anteil mit $4 \%$ geringer ${ }^{227}$, aber noch immer im nachweisbaren Bereich. Für die Überschallexpansionen kann dabei möglicherweise ein hiervon abweichender $5 p$-Anteil erwartet werden, da im Vorfeld nicht eindeutig abschätzbar ist, in wieweit die Tautomerisierung im Nichtgleichgewichtssystem der Überschallexpansionen dem flüssigen Zustand in der Probenkammer oder dem gasförmigen entspricht.

Abbildung 5.5 zeigt die auf MP2/aug-cc-pVDZ-Niveau erhaltenen Strukturen der beiden Phenylimidazoltautomere sowie ihrer gemischten Dimere mit Wasser. Für das 4-Phenylimidazol ist eine annähernd planare Struktur zu erkennen, so dass von einer stabilisierenden Konjugation der $\pi$-Systeme der beiden aromatischen Untereinheiten auszugehen ist. Für das 5-Phenylimidazol ist hingegen eine deutliche Torsion der Ringstrukturen zueinander von etwa $32^{\circ}$ zu erkennen, die vermutlich auf die an beiden Seiten auftretenden sterischen Wechselwirkungen zweier Wasserstoffatome der Phenyl- und der Imidazolylgruppen zurückzuführen ist. Anzeichen für eine solche Torsion wurden auch experimentell mittels elektronischer Spektroskopie erhalten. ${ }^{205} \mathrm{Im}$ 4-Phenylimidazol hingegen tritt ein solcher, energetisch ungünstiger geringer Wasserstoff-Wasserstoff-Abstand nur einmal auf, so dass zwar keine Abweichung von der Planarität des Moleküls entsteht, hierdurch jedoch die eher geringe energetische Bevorzugung des $4 p$-Tautomers von $3.8 \mathrm{~kJ} \mathrm{~mol}^{-1}$ gegenüber $5 p$ nachvollziehbar wird.

Abbildung 5.6 a zeigt das Spektrum der Popcorn-Jet-Expansion von Phenylimida- 


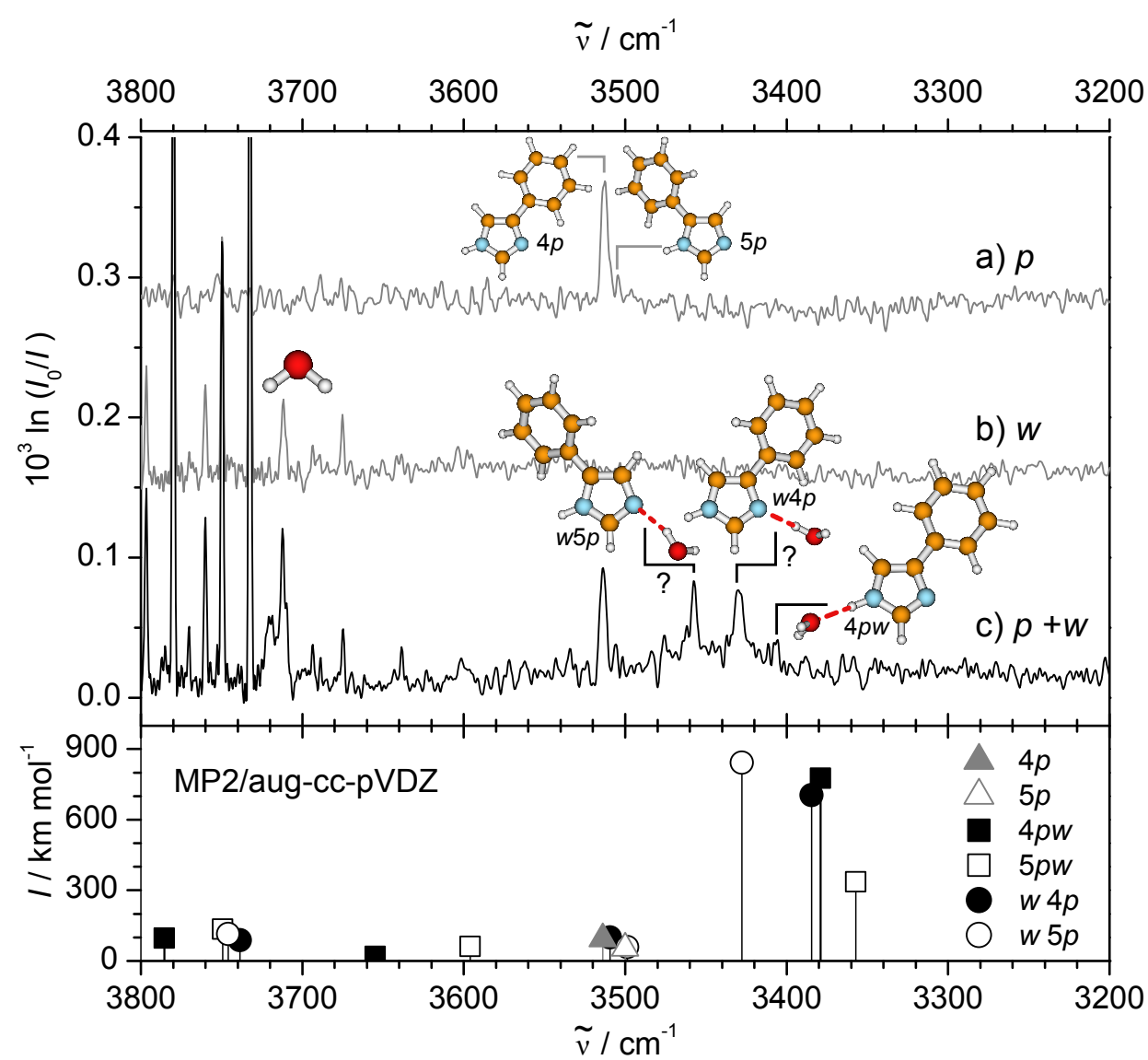

Abbildung 5.6: Spektren der Popcorn-Jet-Expansionen von a) 4-/5-Phenylimidazol, b) Wasser und c) beider Substanzen. Trägergas: Helium, $p_{0}=1.5$ bar. Theoretische Linienspektren wurden aus harmonischen MP2/aug-cc-pVDZ-Rechnungen nach Verschiebung um $-144 \mathrm{~cm}^{-1}$ analog zu den Imidazolspektren in Abbildung 5.4 erhalten. Die Molekül- und Aggregatbenennung entspricht der aus Abbildung 5.5.

zol $\left(\vartheta=160^{\circ} \mathrm{C}\right)$ in Helium bei einem Stagnationsdruck von 1.5 bar. Zu erkennen sind zwei Banden bei 3513 und $3505 \mathrm{~cm}^{-1}$ in einem Intensitätsverhältnis von $>10: 1$. Die stärkere Bande bei $3513 \mathrm{~cm}^{-1}$ ähnelt in ihrer Wellenzahl der des Imidazol-Monomers $\left(3518 \mathrm{~cm}^{-1}\right)$ und ist dem Monomer des 4-Phenylimidazols zuzuordnen, während die schwächere Bande bei $3505 \mathrm{~cm}^{-1}$ zum 5-Phenylimidazol gehört. ${ }^{206}$ Das sich mit den berechneten absoluten Bandenstärken von $94 \mathrm{~km} \mathrm{~mol}^{-1}(4 p)$ und $54 \mathrm{~km} \mathrm{~mol}^{-1}(5 p)$ ergebende Anzahlverhältnis von >6:1 weist dabei auf eine mögliche Abweichung des Tautomerenverhältnisses in der Überschallexpansion von dem der Gleichgewichtsgasphase $(24: 1)^{227}$ hin.

Für die Koexpansion wurde die Wasserkonzentration erneut so eingestellt, dass im reinen Wasserspektrum 5.6 b praktisch nur Banden des Monomers erkennbar sind. Im Koexpansionsspektrum c) von Phenylimidazol und Wasser sind zusätzlich zu den je- 
weiligen Monomerbanden drei intensive Banden bei 3720 , 3458 und $3430 \mathrm{~cm}^{-1}$ sowie möglicherweise eine schwache Bande bei $3407 \mathrm{~cm}^{-1} \mathrm{zu}$ sehen. Hierbei ist die Position von $3720 \mathrm{~cm}^{-1}$ typisch für die Schwingungen freier $\mathrm{O}-\mathrm{H}$-Bindungen von Wasser-Donormolekülen in wi-Aggregaten ${ }^{218}$ und auch die Bande bei $3458 \mathrm{~cm}^{-1}$, deren Wellenzahl exakt der der wi-Bande in Abschnitt 5.1 entspricht, weist auf das Vorliegen von $w p$ Aggregaten mit ähnlicher Bindungsgeometrie wie im wi-Dimer hin. Die Zuordnung zu einem Tautomer kann allein aufgrund der experimentellen Daten jedoch nicht getroffen werden.

Vergleichbare Koexpansionen von 4-Phenylimidazol und Wasser haben in RIDIRSpektren nur eine Heterodimerbande bei $3409 \mathrm{~cm}^{-1}$ ergeben, die dem N-H-Donor-Aggregat $4 p w$ zugeordnet wurde. ${ }^{206}$ Hinweise auf das exklusive Vorliegen dieses Heterodimers erbrachten auch R2PI- („Resonant 2 Photon Ionization“) Spektren. ${ }^{204,206}$ Aufgrund der guten Übereinstimmung der N-H-Streckschwingungsbanden der Phenylimidazol-Monomere aus RIDIR-Spektren mit den hier vorgestellten mit einer Abweichung von jeweils nur $1 \mathrm{~cm}^{-1}$ ist auch für die Aggregatbanden von einer ähnlich geringen Diskrepanz auszugehen. Entsprechend könnte die schwache Bande bei $3407 \mathrm{~cm}^{-1}$ im Koexpansionsspektrum $5.6 \mathrm{c}$ dem $4 p w$-Dimer zugeordnet werden, wobei eine Identifikation dieses Peaks als tatsächliche Bande aufgrund des geringen Signal-zu-Rauschen-Verhältnisses nur unter Vorbehalt erfolgen kann. Interessant ist hierbei, dass das 4pw-Aggregat in den Popcorn-Jet-Expansionen offenbar nur einen sehr geringen Anteil der Heterodimere ausmacht, während die zugehörige Bande in den RIDIR-Spektren die stärkste (und einzige) Mischaggregatbande darstellt. ${ }^{206}$ Neben diesem bemerkenswerten Unterschied liefert die in Referenz 206 getroffene Zuordnung auch einen Anhaltspunkt für die weitere Spektreninterpretation.

Für eine Zuordnung der stärkeren Heterodimerbanden von Phenylimidazol und Wasser ist ein Vergleich mit den Ergebnissen quantenchemischer Rechnungen vonnöten. Abbildung 5.5 zeigt die erhaltenen Minimumstrukturen der gemischten Dimere beider Tautomere auf MP2/aug-cc-pVDZ-Niveau. Die nullpunktskorrigierten Dissoziationsenergien $D_{0}$ wurden durch Subtraktion der Monomerenergien von Wasser und dem jeweils enthaltenen Phenylimidazol-Tautomer erhalten, so dass für einen energetischen Vergleich der Heterodimere untereinander gegebenenfalls $3.8 \mathrm{~kJ} \mathrm{~mol}^{-1}$ für die $5 p$-Strukturen abgezogen werden müssen.

Bei den 4-Phenylimidazol-Wasser-Dimeren, die aufgrund des Monomerverhältnisses in Spektrum 5.6 a das Koexpansionsspektrum dominieren sollten, ist das Aggregat $w 4 p$ mit Wasser als Donor gegenüber dem Wasser-Akzeptor-Cluster um $3.4 \mathrm{~kJ} \mathrm{~mol}^{-1}$ stabiler. Interessanterweise bewirkt die Anbindung eines Wassermoleküls an das 3-NStickstoffatom des Imidazolylrings eine Aufhebung der Planarität des 4-Phenylimidazols, der Torsionswinkel zwischen den beiden aromatischen Untereinheiten beträgt $19^{\circ}$. Dies könnte darauf zurückzuführen sein, dass - ähnlich zum 5-Phenylimidazol eine sterische Wechselwirkung zwischen dem Wasser und dem nächstgelegenen Phenyl-Wasserstoffatom auftritt. Der energetische Nachteil durch Verlust der Konjugation beider aromatischer Systeme wird dabei vermutlich teilweise durch eine zusätzli- 
che stabilisierende $\mathrm{C}-\mathrm{H} \cdots \mathrm{O}$-Wechselwirkung zwischen einem Phenyl-C-H und einem freien Elektronenpaar des Wasser-Sauerstoffs kompensiert. Im Isomer $4 p w$ tritt hinge-

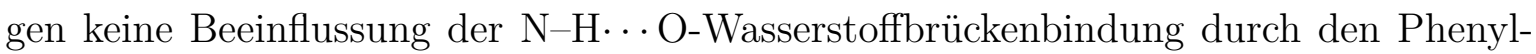
ring auf, so dass ein vergleichbares Verhalten zum analogen Imidazol-Wasser-Dimer $i w$ zu erwarten ist. Entsprechende Strukturen für $w 4 p$ und $4 p w$ wurden auch, allerdings praktisch isoenergetisch, auf B3LYP/6-31+G*-Niveau erhalten. ${ }^{206}$ Rechnungen auf MP2/6-31G*-Niveau fanden ebenfalls vergleichbare Strukturen ${ }^{204}$, hier jedoch mit einer energetischen Bevorzugung des $4 p w$-Isomers, die sich mit den Beobachtungen der unzureichenden Beschreibung dieser Systeme bei Verwendung kleiner Basissätze aus Abbildung 5.1 in Abschnitt 5.1 deckt. Allerdings wurde mit dieser Methode eine zusätzliche lokale Minimumstruktur mit Wasser als Donor, jedoch ohne Deformation des planaren 4-Phenylimidazols gefunden, die durch die hier durchgeführten MP2/aug-ccpVDZ-Rechnungen nicht reproduziert werden konnte.

Da die Wasserstoffbrückenbindung im Aggregat 4pw nicht durch den Phenylsubstituenten beeinflusst sein sollte, ist auch ein dem Cluster $i w$ sehr ähnliches Spektrum zu erwarten. Dies wird durch den Vergleich der Wellenzahl der 4pw-N-H-Streckschwingung von $3407 \mathrm{~cm}^{-1}\left(3409 \mathrm{~cm}^{-1}\right.$ aus RIDIR-Spektren $\left.{ }^{206}\right)$ mit der korrigierten Wellenzahl für $i w$ in Helium-Nanotröpfchen ${ }^{218,219}$ von $3418 \mathrm{~cm}^{-1}$ (siehe auch Tabelle 5.1) bestätigt. Da die entsprechende Bande allenfalls mit einer Intensität knapp über dem Rauschlevel im Koexpansionsspektrum 5.6 c auftritt, wird dieses Aggregat offenbar höchstens zu sehr geringen Anteilen in den Popcorn-Jet-Expansionen gebildet.

Anders verhält es sich für das Heterodimer $w 4 p$, bei dem die Torsion des 4-Phenylimidazols und die zusätzliche $\mathrm{C}-\mathrm{H} \cdots \mathrm{O}$-Wechselwirkung zu einer Beeinflussung der $\mathrm{O}-\mathrm{H} \cdots \mathrm{N}$-Streckschwingung im Vergleich zum wi-Dimer führen sollten. Dies wird auch durch das berechnete Linienspektrum in Abbildung 5.6 bestätigt, wo für beide 4-Phenylimidazol-Wasser-Dimere ähnliche Wellenzahlen $\left(\Delta \tilde{\nu}=6 \mathrm{~cm}^{-1}\right)$ vorausgesagt werden, im Gegensatz zu einer (experimentellen ${ }^{218}$ und theoretischen) Differenz von etwa $40 \mathrm{~cm}^{-1}$ für wi und $i w$. Während eine Zuordnung des $w 4 p$-Dimers zur Bande bei $3458 \mathrm{~cm}^{-1}$ also auszuschließen ist, wäre die Abweichung zwischen Theorie und Experiment für die $3430 \mathrm{~cm}^{-1}$-Bande noch im akzeptablen Bereich. Das Auftreten von $w 4 p$ im Spektrum wird außerdem durch die Stabilität des Aggregats sowie die hohe Häufigkeit des $4 p$-Tautomers im Spektrum 5.6 a der reinen Expansion von Phenylimidazol unterstützt.

Bei den berechneten Strukturen der 5-Phenylimidazol-Wasser-Aggregate in Abbildung 5.5 fällt zunächst auf, dass im Hinblick auf die Dissoziationsenergien hier das $\mathrm{N}-\mathrm{H}-$ Donor-Isomer $5 \mathrm{pw}$ die energetisch bevorzugte Struktur darstellt. Dies ist vermutlich auf die $\mathrm{O}-\mathrm{H} \cdots \pi$-Wechselwirkung zurückzuführen, die eine Stabilisierung des Clusters bewirkt. Das zugehörige berechnete Linienspektrum in Abbildung 5.6 zeigt jedoch, dass diese zusätzliche Wechselwirkung zu einer weiteren Rotverschiebung der $\mathrm{N}-\mathrm{H}$-Streckschwingungsbande führt. Aufgrund der großen Wellenzahldifferenz zu den tatsächlich im Koexpansionsspektrum c) von Phenylimidazol und Wasser beobachteten 
Banden ist ein Vorliegen signifikanter Mengen des Heterodimers $5 p w$ in der gemischten Expansion unwahrscheinlich.

Stellt 5-Phenylimidazol den Wasserstoffbrückenakzeptor im gemischten Dimer mit Wasser dar, ergibt sich hierfür zwar eine geringere Dissoziationsenergie, jedoch auch eine durch die Phenylgruppe praktisch ungestörte $\mathrm{O}-\mathrm{H} \cdots \mathrm{N}$-Bindung, wie sie für einen der Bande bei $3458 \mathrm{~cm}^{-1}$ zuzuschreibenden Cluster zu erwarten ist. Tatsächlich ergibt sich für das w5p-Aggregat in Abbildung 5.6 auch die geringste Differenz zwischen der simulierten intensiven Streckschwingungsbande und der gemessenen Bande bei $3458 \mathrm{~cm}^{-1}$, so dass eine Zusammengehörigkeit von Aggregat und Bande sinnvoll erscheint. Dagegen spricht allerdings die geringe Intensität der Bande des 5-Phenylimidazol-Monomers bei $3505 \mathrm{~cm}^{-1}$ sowohl im Einzelsubstanzspektrum 5.6 a als auch im Koexpansionsspektrum 5.6 c, die eine so starke Heterodimerbande, wie sie bei $3548 \mathrm{~cm}^{-1}$ beobachtet werden kann, nahezu ausschließt. Für eine Begünstigung des Tautomerisierungsprozesses durch Aggregation mit Wasser sind praktisch nur Mechanismen denkbar, die mehrere Wassermoleküle beinhalten. Dies ist aufgrund der geringen Wasserkonzentration im Spektrum c) ebenfalls eher unwahrscheinlich.

Als Alternative für die Deutung der Bande bei $3458 \mathrm{~cm}^{-1}$ kommt allerdings noch eine zusätzliche $w 4 p$-Struktur mit planarem 4-Phenylimidazol, wie sie in MP2/6-31G*Rechnungen $^{206}$ gefunden wurde, in Frage. Hier bestünde ebenfalls eine relativ ungestörte $\mathrm{O}-\mathrm{H} \cdots \mathrm{N}$-Wasserstoffbrückenbindung, die mit den in Abschnitt 5.1 erhaltenen Ergebnissen konform ginge. Diese Hypothese wird ebenfalls durch die höhere Häufigkeit von $4 p$ gegenüber $5 p$ unterstützt.

Auch wenn also für das System 4-/5-Phenylimidazol/Wasser keine eindeutige Strukturzuweisung für die beobachteten Heterodimerbanden möglich ist, sprechen Vergleiche mit Imidazol-Wasser-Koexpansionen, ab initio-Rechnungen und literaturbekannten „Resonant Ion Dip Infrared“-Spektren ${ }^{206}$ für eine Präferenz von Strukturen mit Wasser als Wasserstoffbrückendonor, analog zu den Imidazol-Wasser-Dimeren in Abschnitt 5.1. Dieses Resultat widerspricht der ebenfalls in Überschallexpansionen von Phenylimidazol und Wasser in Helium erhaltenen Präferenz einer Akzeptorrolle des Wassers. ${ }^{204,206}$ Eine Erklärung hierfür könnte der fehlende lineare Zusammenhang zwischen Häufigkeit und Bandenstärke für resonanzbasierte Methoden liefern, durch den eine bevorzugte Detektion des $4 p w$-Dimers möglich wäre. Als Modellsystem für Histidin hat sich Phenylimidazol jedenfalls als nur partiell geeignet erwiesen, da es zwar nötige Strukturmerkmale aufweist, der Phenylsubstituent sich jedoch als zu stark in intermolekulare Wechselwirkungen involviert erwiesen hat um eine ideale Vergleichbarkeit zu Histidin zu erzielen.

\subsection{Zusammenfassung und Ausblick}

Für das System Imidazol-Wasser wurde eine klare Wechselwirkungspräferenz mit Wasser als Wasserstoffbrückendonor gefunden, die analog für Methanol zwar auch existiert, 
jedoch schwächer ausgeprägt ist. Es hat sich weiterhin gezeigt, dass im Gegensatz zur kinetisch kontrollierten Clusterbildung in kryogenen Matrizen ${ }^{213}$ und Lösungsmitteln ${ }^{218}$ die Aggregation in den vorgestellten Überschallstrahlexperimenten überwiegend thermodynamisch kontrolliert verläuft. Dies ist mechanistisch bemerkenswert und spricht für Wasseraustauschprozesse in den frühen Stadien der Expansionen. Die gefundene energetische Reihenfolge der Imidazol-Wasser-Heterodimere kann daher als Benchmark für neue und verbesserte intermolekulare Imidazol- ${ }^{228}$ und Histidin-Kraftfelder Verwendung finden.

Simulationen der gemischten Dimere mittels quantenchemischer Rechnungen haben die Notwendigkeit größerer Basissätze für eine hinreichende energetische Beschreibung, insbesondere bei MP2-Rechnungen, gezeigt. Für qualitative Struktur-, Energie- und Frequenzvorhersagen haben sich harmonische MP2/aug-cc-pVDZ-Rechnungen jedoch als ausreichend gezeigt, obwohl ihnen lediglich zwei Basisfunktionen pro Valenzorbital zugrunde liegen und weder eine Korrektur des Basissatzüberlagerungsfehlers noch eine Berücksichtigung anharmonischer Effekte beinhaltet ist.

Mittels FTIR-Spektroskopie im Überschallstrahl ist es also möglich, isolierte Wechselwirkungen zwischen Aminosäureseitenketten und einzelnen Wassermolekülen, wie sie in Enzymen auftreten 7,8 , zu modellieren. Im Gegensatz zu anderen experimentellen Ansätzen wie der IR/UV-Doppelresonanz ${ }^{229}$ ist hierfür keine zusätzliche Chromophoreinheit im Molekül notwendig.

Für die Einbeziehung von Tautomeriephänomenen in die Simulation von HistidinWasser-Wechselwirkungen in Enzymen hat sich Phenylimidazol als nur eingeschränkt verwendbar erwiesen, da aufgrund der Konjugation der beiden aromatischen Untereinheiten und der zusätzlichen $\mathrm{C}-\mathrm{H} \cdots \mathrm{O}$ - und $\mathrm{O}-\mathrm{H} \cdots \pi$-Wechselwirkungen zu starke Effekte auf die Aggregatenergien zu erwarten sind. Während 4-/5-Phenylimidazol daher zwar ein interessantes System für die Untersuchung konkurrierender Wechselwirkungen bei der Aggregation mit Wasser ist, stellen möglicherweise alkylierte Imidazolderivate die besseren Modelle für Histidin dar, auch wenn sich hier ebenfalls zusätzliche $\mathrm{C}-\mathrm{H} \cdots$ O-Interaktionen sowie die für Berechnungen ungünstige Vielfalt an Konformationen als problematisch erweisen können.

Insgesamt hat sich die Popcorn-Jet-Apparatur jedoch als gut geeignet für die Untersuchung konkurrierender Wechselwirkungen schwerflüchtiger Substanzen mit Lösungsmittelmolekülen erwiesen, so dass eine Übertragung auf weitere gemischte Wasser-Imidazolderivat-Cluster lohnend erscheint. Ein interessantes System hierfür könnte das biogene Amin Histamin (2-(4-/5-Imidazolyl)-ethylamin) ${ }^{194}$ liefern, das im menschlichen Organismus an zahlreichen physiologischen und pathologischen Prozessen beteiligt ist. ${ }^{230}$ Aufgrund seiner beiden Tautomere, der flexiblen Seitenkette sowie der Aminogruppe sollte es in der Lage sein, bei der Aggregation mit Wasser eine Vielzahl von Wechselwirkungen einzugehen. ${ }^{231}$ Die Bestimmung des präferierten Interaktionstyps kann dabei aufschlussreich für das Verständnis der Hierarchien innerhalb konkurrierender Wechselwirkungen sein. 


\section{Infrarotspektroskopische Bildgebung von Überschallexpansionen}

\subsection{Distickstoffmonoxid $\left(\mathrm{N}_{2} \mathrm{O}\right)$}

Distickstoffmonoxid ( $\mathrm{N}_{2} \mathrm{O}$, Lachgas) ist unter Standardbedingungen ein farbloses, süßlich riechendes Gas. ${ }^{232}$ Seine Konzentration in der Atmosphäre beträgt etwa 320 ppb, wo es infolge seiner stark infrarotaktiven Schwingungsmoden als effizientes Treibhausgas wirkt. ${ }^{233}$ Gleichzeitig eignet es sich aufgrund seiner hohen Bandenintensitäten ausgezeichnet als Testsubstanz für die Charakterisierung neuer Apparaturen ${ }^{57}$ und besitzt zudem Schwingungsmoden im mit dem Chilli-Jet untersuchbaren Spektralbereich zwischen 3000 und $800 \mathrm{~cm}^{-1}$ (siehe Tabelle 6.1). Aufgrund der ausgeprägten P- und RZweig-Struktur der Monomerbanden und der linearen Molekülgeometrie kann zudem auch bei geringer spektraler Auflösung eine Untersuchung der Rotationstemperaturen mithilfe Gleichung 2.11 (S. 16) erfolgen.

Neben den Fundamentalschwingungen eigenen sich auch viele Kombinations- und Obertonbanden für die schwingungsspektroskopische Untersuchung. ${ }^{236,237}$ Insbesondere die $\nu_{1}+\nu_{3}$ Kombinationsbande ist ausführlich untersucht worden, da sie sich als gut geeignet für Clustertemperaturanalysen erwiesen hat. ${ }^{238}$ Mit ihrer Schwingungswellenzahl von $3481.2 \mathrm{~cm}^{-1}$ liegt sie jedoch aufgrund der Einschränkung durch das Infrarotobjektiv außerhalb des mit dem Chilli-Jet untersuchbaren Bereichs.

Gasförmiges Distickstoffmonoxid ist schwingungsspektroskopisch über weite Bereiche vom fernen ${ }^{239}$ über das mittlere ${ }^{240}$ bis zum nahen Infrarot ${ }^{241}$ und mit einem breiten Methodenspektrum reichend von Überschallexpansionen ${ }^{10,41,57}$ über Kollisionsküh-

\begin{tabular}{lccc}
\hline & $\nu_{1}$ & $\nu_{2}$ & $\nu_{3}$ \\
\hline \hline Typ & symmetrische & Knick- & asymmetrische \\
Entartung & Streckschwingung & schwingung & Streckschwingung \\
Wellenzahl $^{234}$ & 1 & 2 & 1 \\
Symmetrie $^{232}$ & $\Sigma^{+}$ & $588.77 \mathrm{~cm}^{-1}$ & $2223.76 \mathrm{~cm}^{-1}$ \\
Exp. integrierte & $60 \mathrm{~km} \mathrm{~mol}^{-1}$ & $8.2 \mathrm{~km} \mathrm{~mol}^{-1}$ & $350 \mathrm{~km} \mathrm{~mol}^{-1}$ \\
Bandenstärke $^{235}$ & & & \\
\hline
\end{tabular}

Tabelle 6.1: Schwingungseigenschaften von Distickstoffmonoxid. 
a)

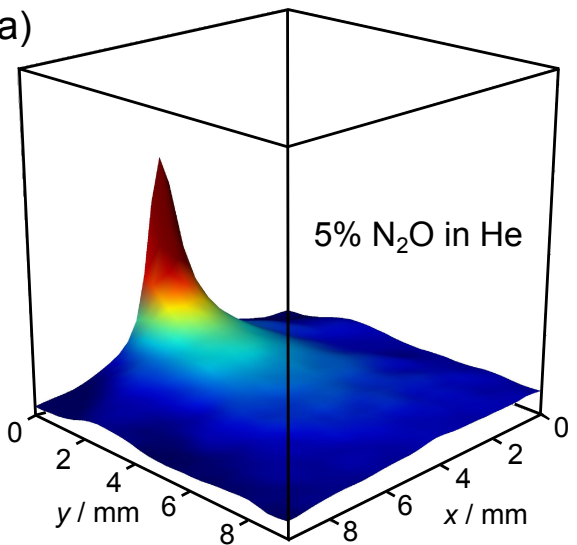

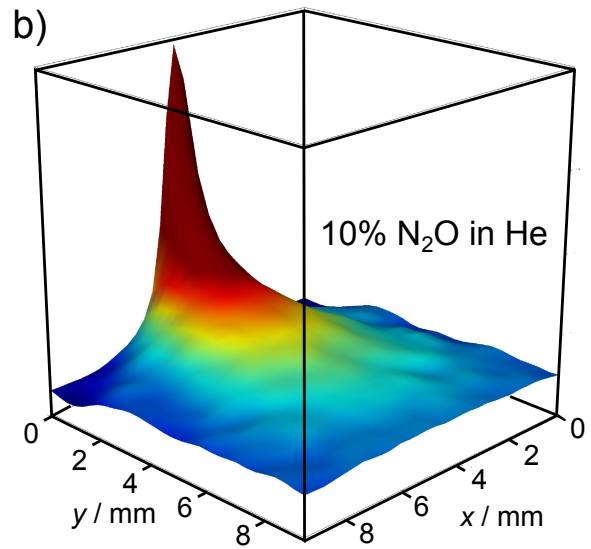

Abbildung 6.1: Dichteverlauf gepulster Expansionen von Distickstoffmonoxid in Helium. Die $x$ - und $y$-Koordinaten geben die räumliche Position in der Expansion wieder, Düsenausgang bei $x \approx 5 \mathrm{~mm}, y \approx 0 \mathrm{~mm}$. Die $z$-Werte entsprechen dem Integral über die gesamte $\nu_{3}$-Bande von $\mathrm{N}_{2} \mathrm{O}$. a) Expansion von $5 \% \mathrm{~N}_{2} \mathrm{O}$ in He. b) Expansion von $10 \% \mathrm{~N}_{2} \mathrm{O}$ in He. $p_{0}=1$ bar, $p_{\mathrm{BG}}<0.2$ mbar, gleiche Skalierung der $z$-Achsen.

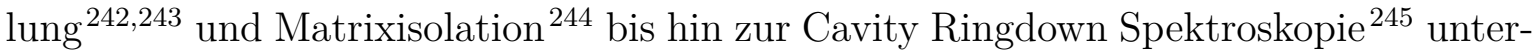
sucht. Auch die Spektren kleiner ${ }^{244,246,247,248,249}$ und großer ${ }^{10,41,242,243,246}$ Lachgasaggregate sind entsprechend gut bekannt. Sie werden aufgrund des kleinen Dipolmoments des Monomers ${ }^{232}$ von $0.161 \mathrm{D}=5.37 \cdot 10^{-31} \mathrm{Cm}$ und trotz des mäßig großen Quadrupolmoments ${ }^{250}$ von $-11.22 \cdot 10^{-40} \mathrm{Cm}^{2}$ überwiegend aufgrund von Dispersionswechselwirkungen gebildet. ${ }^{251}$ Ebenso sind auch die Spektren gemischter Aggregate mit anderen kleinen Molekülen oder Atomen wie Wasser ${ }^{252}$ oder Helium ${ }^{253}$ bereits untersucht. Durch die geringen elektrostatischen Anteile an den intermolekularen Wechselwirkungen von Lachgas sind die Cluster eher schwach gebunden. So beträgt die experimentell bestimmte Dimerisierungsenergie ${ }^{254}$ mit $5 \mathrm{~kJ} \mathrm{~mol}^{-1}$ nur gut ein Drittel des Werts von Wasser $^{159}$.

Aufgrund dieser Kombination von Moleküleigenschaften aus stark infrarotaktiven Banden, einfacher Temperaturanalyse auch bei geringer spektraler Auflösung und der Ausbildung von Molekülaggregaten im Jet wird Distickstoffmonoxid hier als Testsystem für den neuen Aufbau zur chemischen Bildgebung von Überschallexpansionen verwendet.

\subsection{Gepulste Expansionen}

Abbildung 6.1 zeigt den Dichteabfall gepulster Expansionen von Distickstoffmonoxid in Helium mit verschiedenen $\mathrm{N}_{2} \mathrm{O}$-Konzentrationen bei einem Stagnationsdruck von 1 bar und einem geringen Hintergrunddruck von maximal 0.2 mbar. Als Maß für die Dichte wurde das Integral über die gesamte Bande der antisymmetrischen Streckschwingung 
des Distickstoffmonoxids gewählt und als Funktion des Ortes innerhalb der Expansion dargestellt. Hierbei wurde der Effekt unterschiedlicher Bandenstärken der Schwingungen von freien und in Clustern gebundenen $\mathrm{N}_{2} \mathrm{O}$-Molekülen vernachlässigt, da die Intensitätsverstärkung durch Aggregation im Gegensatz zu wasserstoffbrückengebundenen Systemen gering ist. ${ }^{251}$ Direkt hinter der Schlitzdüse $(x \approx 5 \mathrm{~mm}, y \approx 0 \mathrm{~mm})$ ist die Lachgasdichte am höchsten, sinkt mit steigendem Düsenabstand jedoch schnell auf einen Wert nur leicht oberhalb der Dichte des Hintergrundgases - erkennbar an den Bildecken für kleine $y$-Werte - ab. Dieser schnelle Dichteabfall veranschaulicht gut die stark verdünnten Gasphasenbedingungen innerhalb von Überschallexpansionen. Der stufenartige Dichteabfall seitlich des Düsenausgangs markiert die Grenze der Expansion.

Obwohl der $\mathrm{N}_{2} \mathrm{O}$-Anteil in der Gasmischung in Abbildung $6.1 \mathrm{~b}$ mit $10 \%$ eigentlich doppelt so hoch sein sollte wie in 6.1 a wird für das $\nu_{3}$-Bandenintegral direkt hinter der Schlitzdüse ein Wert erreicht, der nur etwa 1.8mal so hoch ist. Die Abweichung vom idealen Integralverhältnis kann einerseits auf unpräzise Druckverhältnisse bei der Vormischung von Lachgas und Helium sowie auf eine unvollständige Durchmischung der Gase innerhalb der Druckgasflaschen zurückgeführt werden. Andererseits kann bei der hohen $\mathrm{N}_{2} \mathrm{O}$-Konzentration direkt hinter der Düse in Kombination mit der großen Infrarotintensität der $\nu_{3}$-Schwingung der Transmissionsgrad des Infrarotlichts so stark absinken, dass Streulichteffekte und Artefakte überwiegen und so zu geringe Absorbanzwerte erhalten werden, auch wenn diese aufgrund der geringen spektralen Auflösung von $2 \mathrm{~cm}^{-1}$ eventuell nicht unmittelbar zu erkennen sind. Um das Ausmaß solch eines Effekts einschätzen zu können, soll an dieser Stelle eine Abschätzung der Transmission direkt hinter der Schlitzdüse erfolgen.

Für die Expansion von 10\% Lachgas in Helium weist das Spektrum mit einer Auflösung von $\Delta \tilde{\nu}_{\exp }=2 \mathrm{~cm}^{-1}$ an der Position direkt hinter der Schlitzdüse eine Maximalabsorbanz von $A_{\text {exp }}=\ln \left(\frac{I_{0}}{I}\right) \approx 0.3$ auf. Die Maximalabsorbanz in einem nicht auflösungslimitierten Spektrum ergibt sich dann mit der Anzahl $n_{\text {Banden }}$ von Rotationslinien im betrachteten Bereich von $2 \mathrm{~cm}^{-1}$ mit Halbwertsbreiten (FWHM) von $\Delta \tilde{\nu}_{\mathrm{FWHM}}$ als:

$$
A_{\max }=\frac{A_{\text {exp }} \cdot \Delta \tilde{\nu}_{\exp }}{n_{\text {Banden }} \cdot \Delta \tilde{\nu}_{\mathrm{FWHM}}}
$$

Aus der Rotationskonstanten ${ }^{255} B_{\mathrm{N}_{2} \mathrm{O}} \approx 0.419 \mathrm{~cm}^{-1}$ ergibt sich eine Anzahl von ungefähr 2.5 Rotationslinien pro $2 \mathrm{~cm}^{-1}$-Intervall. Für die Abschätzung der Doppler- und Druckverbreiterung der Banden werden eine Temperatur von $100 \mathrm{~K}$ und ein Druck von 50 mbar direkt hinter dem Düsenausgang angenommen. Die Temperaturbestimmung erfolgte dabei gemäß Gleichung 2.11, der Druck wurde durch Bandenintegralvergleich mit einem $\mathrm{N}_{2} \mathrm{O}$-Gasphasenspektrum bei bekanntem Druck erhalten. So ergibt sich eine Dopplerbreite (FWHM) von ${ }^{100}$

$$
\Delta \tilde{\nu}_{\mathrm{D}}=2 \cdot \frac{\tilde{\nu}}{c} \sqrt{\frac{2 k T \ln 2}{m}} \approx 2 \cdot 10^{-3} \mathrm{~cm}^{-1}
$$


mit der Wellenzahl der Bande $\tilde{\nu}$ und der Masse $m$ von $\mathrm{N}_{2} \mathrm{O}$.

Die Druckverbreiterung kann aus den Summen der Stoßdurchmesser $D_{i}$ der absorbierenden Substanz $\left(\mathrm{N}_{2} \mathrm{O}\right)$ und der möglichen Stoßpartner $\left(\mathrm{He}, \mathrm{N}_{2} \mathrm{O}\right)$ und den Anzahldichten $N_{i}$ der Moleküle gemäß

$$
\Delta \tilde{\nu}_{p}=\frac{1}{4 \pi} \sum_{i}^{i=\mathrm{N}_{2} \mathrm{O}, \mathrm{He}} N_{i} \cdot\left(D_{\mathrm{N}_{2} \mathrm{O}, i}\right)^{2}\left[2 \pi k T\left(\frac{1}{m_{\mathrm{N}_{2} \mathrm{O}}}+\frac{1}{m_{i}}\right)\right]^{0.5}
$$

berechnet werden. ${ }^{256}$

Mit den Stoßdurchmessern ${ }^{257}$ von $0.4 \AA(\mathrm{He})$ und $8.4 \AA\left(\mathrm{N}_{2} \mathrm{O}\right)$ ergibt sich so eine Halbwertsbreite durch Druckverbreiterung von $0.02 \mathrm{~cm}^{-1}$. Diese liegt etwa eine Größenordnung über der Dopplerverbreiterung, so dass für weitere Berechnungen nur der Wert der Druckverbreiterung berücksichtigt werden muss. Für die minimale Transmission ergibt sich mit Gleichung 6.1:

$$
T r_{\min }=\exp \left(-\frac{A_{\text {exp }} \cdot \Delta \tilde{\nu}_{\exp }}{n_{\text {Banden }} \cdot \Delta \tilde{\nu}_{\mathrm{FWHM}}}\right) \approx \exp \left(-\frac{0.3 \cdot 2 \mathrm{~cm}^{-1}}{2.5 \cdot 0.02 \mathrm{~cm}^{-1}}\right) \approx 6 \cdot 10^{-6}
$$

Direkt hinter dem Düsenausgang wird daher in der Spitze praktisch das gesamte Infrarotlicht absorbiert, so dass von einer klaren Beeinträchtigung der Intensitäten auszugehen ist. Hierdurch wird also vermutlich die Diskrepanz zwischen rechnerischem und gemessenem Verhältnis der Bandenintegrale der Expansionen mit 5\% und 10\% $\mathrm{N}_{2} \mathrm{O}$ in Helium an Düsenausgang bedingt. Da sowohl gemäß Gleichung 6.3 die Druckverbreiterung als auch die Absorbanz in etwa linear mit der Anzahldichte abnehmen, bleibt dieses Sättigungsproblem solange bestehen, bis die Bandenbreite durch die Dopplerverbreiterung dominiert wird.

Die ortsaufgelöste Darstellung des Bandenintegrals kann zwar als Maß für den Dichteverlauf verwendet werden, sie macht jedoch keine Aussagen über Änderungen der Bandenformen und enthält daher auch keine Information über Aggregationsprozesse. Die Spektren in den Bereichen der $\nu_{3}$ - und $\nu_{1}$-Fundamentalschwingungen der gepulsten Expansionen von 5\% und 10\% Lachgas in Helium sind in Abhängigkeit vom Abstand zur Düse in Abbildung 6.2 gezeigt. Die einzelnen Spektren stellen dabei Mittelungen über jeweils drei benachbarte der insgesamt dreißig Pixelreihen dar, die orthogonal zur Ausbreitungsrichtung der Expansion verlaufen. Pixel in Regionen außerhalb der Expansion, die nur das Hintergrundgas erfassen, wurden dabei nicht mit einbezogen. Alle Spektren wurden nach Anwendung der in Kapitel 3.4.5 beschriebenen Datennachbehandlung erhalten, im Bereich der symmetrischen Streckschwingungsbande $\nu_{1}$ wurde zusätzlich ein Mittelwertspektrum der Pixel außerhalb der Expansion subtrahiert, um das Signal-zu-Rauschen-Verhältnis zu verbessern. Im $\nu_{3}$-Bereich wurde dieses Verfahren nicht angewendet, da hier aufgrund der höheren Infrarotintensitäten die Bandenstärken des Hintergrundgases deutlich über dem Niveau des pixelübergreifenden Rauschens liegen. 


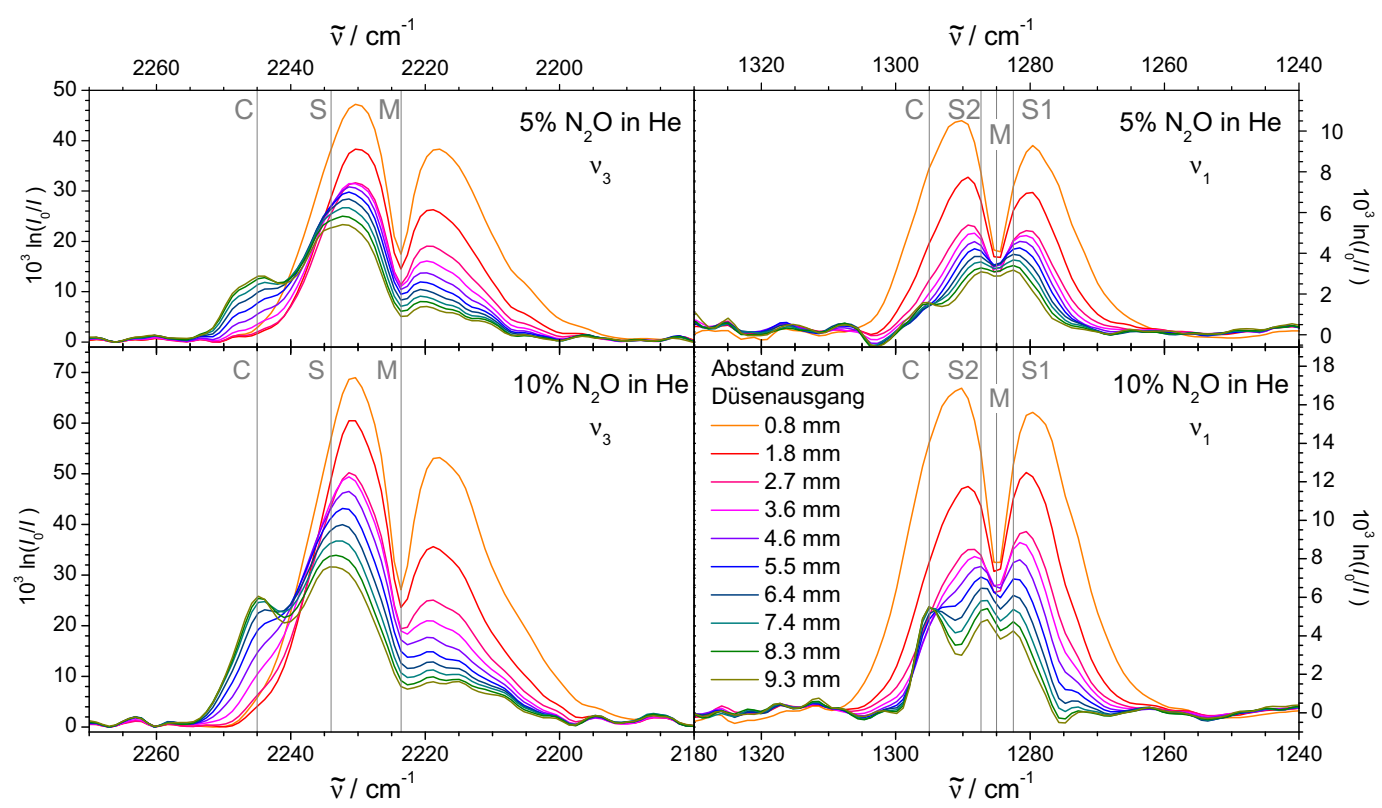

Abbildung 6.2: $\nu_{3}$ - und $\nu_{1}$-Streckschwingungsbanden aus den in Abb. 6.1 gezeigten Expansionen in Abhängigkeit vom Düsenabstand. Die Spektren wurden durch Mittelung von jeweils drei benachbarten Pixelreihen orthogonal zur Ausbreitungsrichtung ohne Berücksichtigung der Bereiche außerhalb der Expansionsgrenzen erhalten. Düsenabstandswerte sind als mittlere $y$-Werte zu verstehen. M: Monomer-Bandenzentrum, C: Clusterkern, S/S1/S2: Clusteroberfläche.

Der Farbverlauf der Spektren in Abbildung 6.2 gibt von orange über rot und blau bis zu grün steigende Abstände zum Düsenausgang wieder. Bei kleinen Abständen (orange Spektren) ist für beide Lachgaskonzentrationen und Spektrenbereiche deutlich die P- und R-Zweig-Struktur der Monomerbanden mit Bandenzentren bei $2224\left(\nu_{3}\right)$ und $1285 \mathrm{~cm}^{-1}\left(\nu_{1}\right)$ erkennbar. Die Monomer-Rotationstemperatur kann mithilfe von Gleichung 2.11 (S. 16) aus der Wellenzahldifferenz der Maxima von P- und R-Zweig und einem Gasphasen-Referenzwert von $\Delta \tilde{\nu}_{\mathrm{PR}}^{300 \mathrm{~K}}\left(\nu_{3}\right) \approx 21 \mathrm{~cm}^{-1}$ auf etwa $80 \mathrm{~K}$ abgeschätzt werden, offenbar findet also bereits innerhalb der Schlitzdüse und während der ersten $0.3 \mathrm{~mm}$ der Expansion, die aufgrund des Glättungsverfahrens nicht vollständig erfasst werden, eine beträchtliche Abkühlung statt. Für größere Düsenabstände lässt die Überlagerung der Monomerzweige mit Aggregatbanden eine derartige Temperaturauswertung nicht zu.

Mit steigendem Düsenabstand erfolgt ein schneller Abfall der Monomerbanden, während zusätzliche Banden bei 2235, 1287 und $1283 \mathrm{~cm}^{-1}$ an Intensität gewinnen. Diese Banden wurden bereits früher Molekülen an der Oberfläche mittelgroßer Aggregate zugeordnet $^{10}$, sie werden daher als S-Banden (surface) bezeichnet. ${ }^{10,41}$ Die Zuordnung erscheint sinnvoll, da sich in der Expansion zunächst kleinere Cluster ausbilden, bei denen nahezu alle Moleküle an der Oberfläche lokalisiert sind. Die Aufspaltung in zwei 


\begin{tabular}{ccccc}
\hline \multirow{2}{*}{ Bande } & \multicolumn{2}{c}{$\nu_{\mathbf{3}} / \mathrm{cm}^{-1}$} & \multicolumn{2}{c}{$\nu_{\mathbf{1}} / \mathrm{cm}^{-1}$} \\
& Chilli-Jet & Literatur & Chilli-Jet & Literatur \\
\hline \hline M & 2224 & $2223.76^{234}$ & 1285 & $1284.91^{234}$ \\
C & 2245 & $2244.3^{41}$ & 1296 & $1295^{41}$ \\
S & 2235 & $2235.4^{41}$ & & \\
S1 & & & 1283 & $1282.6^{41}$ \\
S2 & & & 1287 & $1286.7^{41}$ \\
\hline
\end{tabular}

Tabelle 6.2: Bandenpositionen der Streckschwingungen von $\mathrm{N}_{2} \mathrm{O}$, Vergleich der Chilli-JetMessungen mit Literaturwerten. Die Bandenbezeichnungen entsprechen denen in Abbildung 6.2 .

Banden im $\nu_{1}$-Bereich ist vermutlich auf eine unterschiedliche Orientierung der Oberflächenmoleküle zurückzuführen ${ }^{41}$ Mit weiter steigendem Abstand werden zwei weitere Banden bei 2245 und $1296 \mathrm{~cm}^{-1}$ erkennbar, die zuvor mit Molekülen im Clusterinneren (C-Banden, $\boldsymbol{c o r e}$ ) in Verbindung gebracht wurden. ${ }^{10,41} \mathrm{Ihr}$ relativ spätes Auftreten in der Expansion bestätigt auch diese Zuordnung. Eine Auflistung der verschiedenen Bandenpositionen und ein Vergleich mit Literaturwerten ist in Tabelle 6.2 gegeben.

Ein Vergleich der Spektren von Expansionen mit 5\% und 10\% Distickstoffmonoxid in Helium zeigt zunächst, dass der prozentuale Abfall der Monomerbandenintensität mit steigendem Düsenabstand bei beiden ähnlich schnell verläuft. Da dieser zum großen Teil auf den allgemeinen Dichteabfall innerhalb der Expansion zurückführbar ist und für beide Expansionen der gleiche Stagnationsdruck verwendet wurde, entspricht dieses Verhalten den Erwartungen. Eine Analyse der Clusterbanden ist insbesondere für die Oberflächenbanden und den $\nu_{1}$-Bereich aufgrund der starken Überlappung mit der Monomer-Zweigstruktur erschwert. Ein Vergleich der Positionen der gemeinsamen Monomer+S-Banden-Maxima mit den Positionen der reinen S-Banden zeigt jedoch, dass für die $\mathrm{N}_{2} \mathrm{O}$-reichere Mischung die Peakpositionen schneller auf den S-Banden-Wert konvergieren als bei den verdünnteren Mischungen. Dies ist besonders gut im Bereich der $\nu_{3}$-Bande zu sehen, da hier die Verschiebung des Gesamtmaximums durch Abkühlung des Monomers (Shift des R-Zweig-Maximums in Richtung Bandenzentrum) der durch Clusterbildung entgegengerichtet verläuft.

Während die Oberflächenbanden bei geringerer Lachgaskonzentration mit steigendem Abstand nur leicht schwächer an Intensität gewinnen als bei höherer Konzentration, ist dieser Effekt für die C-Banden deutlich ausgeprägter und aufgrund ihrer besseren Separierung von den Monomerbanden auch in beiden Bandensystemen gut erkennbar. Verdeutlicht wird dieses verschiedene Verhalten auch in den unterschiedlichen Intensitätsverhältnissen von S- und C-Banden zueinander sowohl im $\nu_{3}$ - als auch im $\nu_{1}$ Bereich. Insgesamt weist dies darauf hin, dass bei gleichen Düsenabständen und relativ zur Monomer-Anfangskonzentration die gebildeten Aggregate bei höherem Lachgasan- 
teil zahlreicher und größer sind. Dies ist auf die geringere Anzahl von $\mathrm{N}_{2} \mathrm{O}-\mathrm{N}_{2} \mathrm{O}$-Stößen in der verdünnteren Expansion zurückzuführen.

Eine Größenabschätzung der gebildeten Cluster ist für große Düsenabstände und somit geringe Beeinträchtigungen der Clusterbandenintensitäten durch Monomerbeiträge aus den relativen Intensitäten von Kern- und Oberflächenbanden möglich. Kunzmann et al. ${ }^{243}$ haben hierbei ein einfaches Abschätzungsverfahren angewendet, das auf folgenden groben Näherungen beruht: Die Cluster werden als kugelförmige Gebilde einheitlicher Dichte mit einem Radius $r$ angesehen. Sie besitzen eine Oberflächenschicht der Dicke einer Elementarzelle von kristallinem Distickstoffmonoxid, $r_{\mathrm{S}}=0.566 \mathrm{~nm}$. Schwingungen von Molekülen innerhalb dieser Oberflächenschicht tragen ausschließlich zur Intensität der S-Banden bei, während Moleküle im Aggregatkern ausschließlich zu den C-Banden beitragen. Es wird weiterhin davon ausgegangen, dass die Oszillatorstärke der Molekülschwingungen nicht von ihrer Position innerhalb des Aggregats abhängt, die Existenz einer Clustergrößenverteilung wird ignoriert und nur eine einzige mittlere Clustergröße angenommen. Für die Bandenanalyse werden dann die einzelnen $\nu_{1}$-Clusterbanden durch Gaußfunktionen angenähert und das zugehörige Bandenintegral $A$ bestimmt. Aus diesen Annahmen folgt:

$$
\frac{A_{\mathrm{S}}}{A_{\mathrm{S}}+A_{\mathrm{C}}}=\frac{A_{\mathrm{S} 1}+A_{\mathrm{S} 2}}{A_{\mathrm{S} 1}+A_{\mathrm{S} 2}+A_{\mathrm{C}}}=\frac{n_{\mathrm{S}}}{n_{\mathrm{ges}}}=\frac{V_{\mathrm{S}}}{V_{\mathrm{ges}}}=\frac{r^{3}-\left(r-r_{\mathrm{S}}\right)^{3}}{r^{3}}=1-\frac{\left(r-r_{\mathrm{S}}\right)^{3}}{r^{3}}
$$

( $n$ : Anzahl, $V$ : Volumen, Index ges: gesamter Cluster)

Eine solche Auswertung ergibt für einen Düsenabstand von $9.3 \mathrm{~mm}$ einen mittleren Clusterradius von $1.3 \mathrm{~nm}$ für die Expansion von $5 \% \mathrm{~N}_{2} \mathrm{O}$ in Helium und $1.9 \mathrm{~nm}$ für einen Distickstoffmonoxidanteil von 10\%. Wird ein aus der Kristalldichte von Lachgas gewonnener Wert von 90 Molekülen in einem Aggregat mit dem Radius $1 \mathrm{~nm}$ zugrunde gelegt $^{243}$ und eine Abweichung der tatsächlichen Oberflächenschichtdicke des Aggregats von der Größe der $\mathrm{N}_{2} \mathrm{O}$-Elementarzelle um etwa $\pm 20 \%$ angenommen, so ergeben sich für die beiden Expansionen beim betrachteten Düsenabstand mittlere Clustergrößen von etwa $200 \pm 100$ und $600 \pm 300 \mathrm{~N}_{2} \mathrm{O}$-Molekülen.

Die Spektren der $\nu_{3}$-Bande in Abbildung 6.2 zeigen, dass trotz einer generell starken Überlagerung der Banden der P-Zweig der Monomerbande und ein Teil der C-Bande separiert vorliegen. Diese können daher zur Bildgebung des Monomer- und Clusterverlaufs in der Expansion herangezogen werden. Abbildung 6.3 zeigt farbskaliert sowohl den Verlauf des Integrals über den hochfrequenten Teil der C-Bande als Maß für die Ausprägung der Aggregation (links) als auch den Verlauf des Integrals über den Monomer-P-Zweig (rechts) jeweils im Vergleich mit der Gesamtdichte (weiße Konturlinien, analog zu Abbildung 6.1). Das Spektrum in der Mitte der Abbildung zeigt exemplarisch die für die Integration herangezogenen Bandenbereiche. Die Farbkodierung reicht von blau für kleine Integralwerte bis zu rot für hohe Werte, für die Expansionen mit 5\% und $10 \% \mathrm{~N}_{2} \mathrm{O}$ in Helium wurde jeweils die gleiche Farbskala verwendet.

Auf der linken Seite von Abbildung 6.3 ist der Verlauf der Aggregatbildung im Vergleich zur Dichteentwicklung gezeigt. Aufgrund der Verwendung der C-Bande wird 

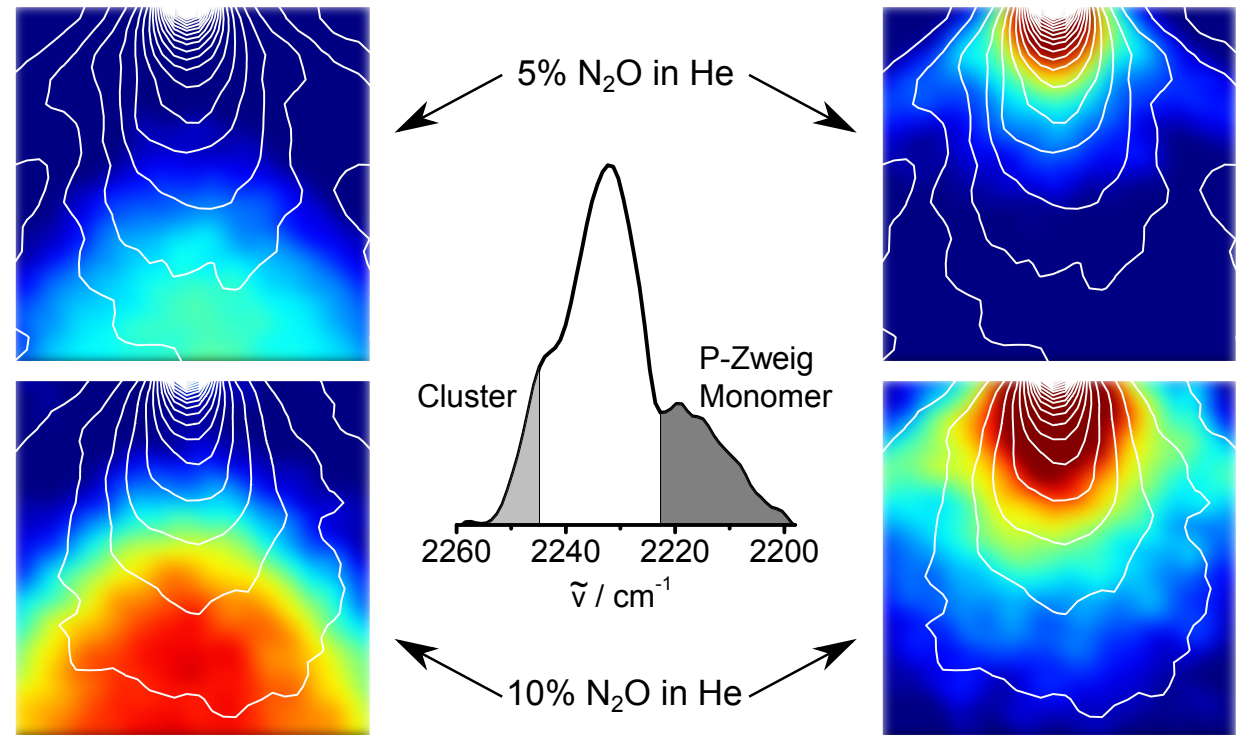

Abbildung 6.3: Entwicklungen der Cluster- und Monomerkonzentrationen im Vergleich zur Gesamtdichte der in Abb. 6.1 gezeigten Expansionen von 5 und $10 \% \mathrm{~N}_{2} \mathrm{O}$ in $\mathrm{He}-$ lium, Schlitzdüsenausgang oben. Mitte: Verwendete Integrationsgrenzen. Links: Integral über separierten Bereich der $\nu_{3}$-C-Bande $\left(2255-2245 \mathrm{~cm}^{-1}\right)$. Rechts: Integral über den Monomer-P-Zweig der $\nu_{3}$-Bande $\left(2223-2180 \mathrm{~cm}^{-1}\right)$. Integralwerte steigen von blau nach rot. Weiße Konturlinien: Verlauf der Gesamtdichte (Integral von 2260$2170 \mathrm{~cm}^{-1}$ ), entspricht Abbildung 6.1. Die Farb- und Konturskalen sind für $5 \%$ und $10 \% \mathrm{~N}_{2} \mathrm{O}$-Gasmischungen identisch.

hier genaugenommen der Bildungs- und Wachstumsverlauf großer Cluster dargestellt. Es zeigt sich, dass der Aggregationsgrad in der Expansion offenbar nicht nur vom Abstand zur Düse, sondern auch vom Winkel relativ zur Ausbreitungsrichtung abhängig ist. Hierbei ist die Clusterbildung an einer Position umso stärker, je weniger sie von der Ausbreitungsebene abweicht. Dies ist einerseits auf die höhere Dichte insbesondere schwererer Komponenten ${ }^{28}$ (hier $\mathrm{N}_{2} \mathrm{O}$ ) im Zentrum der Expansion zurückzuführen. Andererseits bewirken die lateralen Stoßfronten aufgrund der dort vorliegenden höheren Temperaturen eine Reduktion der Aggregation in der Nähe der seitlichen Expansionsgrenzen, so dass beide Effekte zu der betrachteten Winkelabhängigkeit der Clusterbildung beitragen. Wie bereits in den Spektren beobachtet, ist für die verdünntere Gasmischung dabei eine spätere und geringer ausgeprägte Bildung der (großen) Aggregate zu erkennen.

Der Vergleich der Monomerkonzentrationen mit der Gesamtdichte rechts in Abbildung 6.3 zeigt für das Monomer ein Absinken der Konzentration hinter der Schlitzdüse, das schneller verläuft als das der allgemeinen Dichte. Dies weist auf einen signifikanten Verbrauch von $\mathrm{N}_{2} \mathrm{O}$-Monomeren durch die Clusterbildung hin, wie er aufgrund der Größe der gebildeten Aggregate auch zu erwarten ist. Gleichzeitig zeigt die Abbildung 
auch anschaulich die erhöhte Monomerkonzentration an den lateralen Stoßfronten und bestätigt somit den dortigen Zerfall der eher schwach gebundenen Aggregate aufgrund der vorherrschenden höheren Temperaturen.

\subsection{Kontinuierliche Expansionen}

Nachdem die Messungen an gepulsten Systemen gezeigt haben, dass innerhalb der lateralen Stoßfronten offenbar ein Clusterzerfall durch Erwärmung stattfindet, liegt es nahe auch die Auswirkungen der normalen Stoßfront auf das expandierte Gasgemisch zu betrachten. Um diese mit dem eingeschränkten Sichtfeld von $1 \mathrm{~cm}^{2}$ untersuchen zu können ohne Informationen über die Prozesse direkt hinter dem Düsenausgang zu verlieren, muss die Zone freier Expansion durch Variation der experimentellen Parameter verkleinert werden. Hierzu wurde durch eine Verringerung der Öffnung zwischen Expansionsrohr und Puffervolumen die Pumpeffizienz mechanisch verschlechtert und so der Hintergrunddruck erhöht. Um einen konstanten Hintergrunddruck innerhalb der gesamten Messreihe realisieren zu können, wurden zunächst mehrere Hintergrundscans bei evakuiertem Expansionsvolumen aufgenommen. Anschließend wurde das Probegasgemisch kontinuierlich expandiert und erst nach Erreichen eines stabilen Hintergrunddrucks eine entsprechende Anzahl an Probenscans aufgenommen. Für eine weitere Reduktion des Abstands zwischen Düsenausgang und normaler Stoßfront wurde der Stagnationsdruck im Reservoir abgesenkt. Um dennoch ausreichende Bandenintensitäten für Dichte- und Temperaturanalysen zu erhalten, wurde nur mit der höheren Lachgaskonzentration von $10 \%$ gearbeitet und ausschließlich die intensivere $\nu_{3}$-Bande betrachtet.

\subsubsection{Dichteverlauf}

Abbildung 6.4 zeigt das Integral über die $\nu_{3}$-Bande von $\mathrm{N}_{2} \mathrm{O}$ als $\mathrm{Maß}$ für die Lachgasdichte in kontinuierlichen Expansionen von 10\% Distickstoffmonoxid in Helium bei einem Stagnationsdruck von $p_{0}=380$ mbar und unterschiedlichen Hintergrunddrücken. Der Schlitzdüsenausgang befindet sich bei $x \approx 5 \mathrm{~mm}$ und $y \approx 0 \mathrm{~mm}$.

Bei einem relativ niedrigen Hintergrunddruck von 10 mbar (Abb. 6.4 a) ist, wie auch bei den gepulsten Expansionen bereits beobachtet wurde, direkt hinter der Schlitzdüse zunächst ein schneller Dichteabfall und die Ausbildung seitlicher Stoßfronten zu erkennen. Während die Dichte an den lateralen Stoßfronten das Niveau des Hintergrundgases leicht übersteigt, sinkt sie in der Zone freier Expansion auf einen deutlich kleineren Wert ab. An diesen steilen Dichtegradienten schließt sich der etwa $4 \mathrm{~mm}$ lange Bereich der Ruhezone an, der sich durch einen Minimalwert der Dichte innerhalb der Expansion auszeichnet und durch nahezu parallele laterale Stoßfronten begrenzt wird. Bei einem Düsenabstand von etwa $6 \mathrm{~mm}$ erfolgt ein rapider Dichteanstieg, der die Expansion in ihrer Ausbreitungsrichtung begrenzt und einen Wert ähnlich dem 

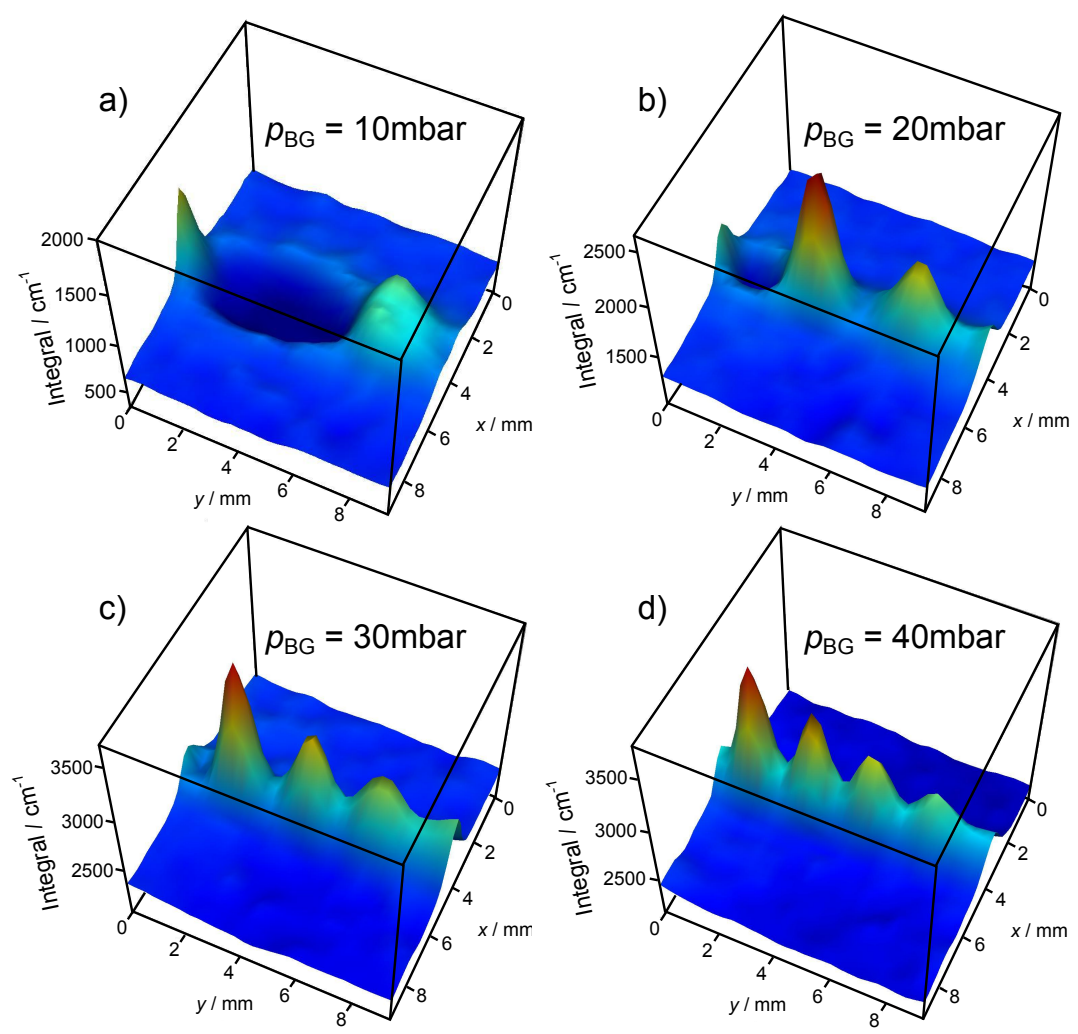

Abbildung 6.4: Dichteverlauf kontinuierlicher Expansionen von 10\% Distickstoffmonoxid in Helium mit $p_{0}=380$ mbar und unterschiedlichen Hintergrunddrücken. Die $x$ - und $y$-Koordinaten geben die räumliche Position in der Expansion wieder, Düsenausgang bei $x \approx 5 \mathrm{~mm}, y \approx 0 \mathrm{~mm}$. Die $z$-Werte entsprechen dem Integral über die gesamte $\nu_{3}$-Bande von $\mathrm{N}_{2} \mathrm{O}$, gleiche Skalierungen der $z$-Achsen nach Höhenanpassung auf die Hintergrunddichte.

am Schlitzdüsenausgang erreicht. Da gemäß Gleichung 2.3 bei der eingesetzten Düsengeometrie und dem vorliegenden Druckverhältnis eine Überkreuzung der lateralen Stoßfronten erst bei deutlich höheren Abständen zur Düse erfolgen sollte, handelt es sich bei dem beobachteten Druckanstieg um die erste normale Stoßfront.

Wird der Hintergrunddruck schrittweise weiter auf 20,30 und 40 mbar erhöht (Abbildungen 6.4 b, c und d), ist zunächst einmal eine Erhöhung des $\nu_{3}$-Integrals im warmen Hintergrundgas zu erkennen. Der Abstand zwischen Schlitzdüsenausgang und normaler Stoßfront sinkt und die Zone freier Expansion wird kürzer. Gleichzeitig wird auch die seitliche Ausbreitung der Expansion aufgrund der höheren Dichte des Hintergrundgases eingeschränkt, die Expansion wird schmaler. Diese Verengung der Ruhezone bestätigt dabei die Beobachtungen bei der Charakterisierung der Multischlitzdüsenexpansionen in Abschnitt 3.2.4, dass nebenliegende Expansionen, die ja auch eine Art seitlichen Hintergrunddruck generieren, eine Verformung der Expansion bewirken. Bei einem Druck von 40 mbar (Abb. 6.4 d) ist die Expansionszone so geschrumpft, dass sie kaum noch 


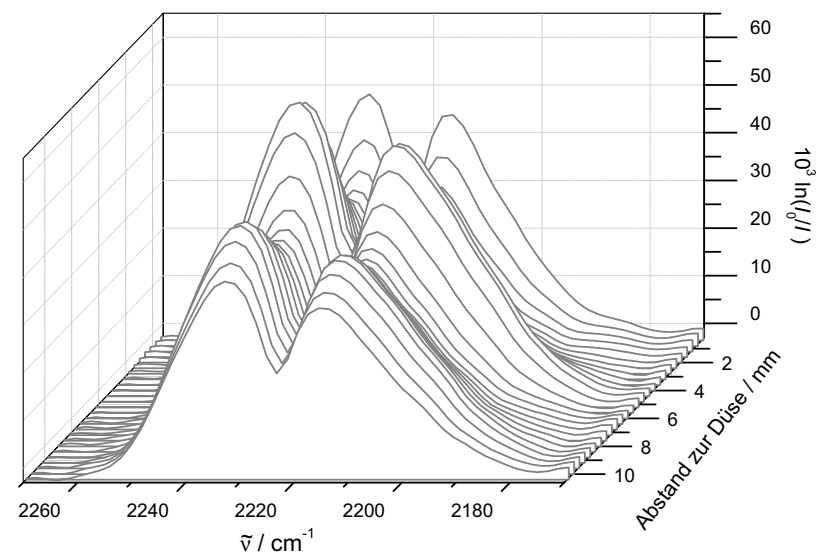

Abbildung 6.5: Entwicklung der $\nu_{3}$-Bande von $\mathrm{N}_{2} \mathrm{O}$ bei der kontinuierlichen Expansion mit 20 mbar Hintergrunddruck aus Abbildung $6.4 \mathrm{~b}$ mit $x \approx 5 \mathrm{~mm}$ und variablem Abstand zur Düse.

erkennbar ist. Da bei steigendem Hintergrunddruck die Expansionszeit bis zum Eintreten der normalen Stoßfront immer geringer wird, erhöht sich auch der Minimalwert der Dichte und unterschreitet teilweise nicht einmal den der Hintergrunddichte. Darüber hinaus sinkt die Dichte direkt hinter der Schlitzdüse ab, so dass der Dichtepeak der ersten frontalen Stoßwelle den am Düsenausgang übersteigt. Dahinter wird eine periodische Struktur aus abwechselnder Expansion und Kompression des Gases sichtbar, wobei mit steigendem Abstand zur Düse die Dichtemaxima in den Kompressionsregionen und die Minima in den Reexpansionen sich immer mehr einem gemeinsamen Wert annähern. Bereits das sekundäre Dichteminimum unterschreitet jedoch schon nicht mehr die Dichte des Hintergrundgases. Insbesondere in Abbildung $6.4 \mathrm{~d}$ ist zusätzlich eine Krümmung des Expansionsverlaufs zu kleinen $x$-Werten zu beobachten. Diese resultiert aus einer Krümmung des Expansionsrohrs zum Puffervolumen.

Während der Dichteverlauf wie in Kapitel 2.3 dargestellt auch mit traditionelleren Methoden wie der Schlierenfotographie untersucht werden kann und wird, bietet der Chilli-Jet-Aufbau zusätzlich den Vorteil chemischer Informationen durch Aufnahme der Schwingungsspektren. Abbildung 6.5 veranschaulicht die Entwicklung der Bande der antisymmetrischen Streckschwingung von $\mathrm{N}_{2} \mathrm{O}$ aus der in Abbildung 6.4 b gezeigten Messung mit einem Hintergrunddruck von 20 mbar. Abgebildet sind die Spektren auf der zentralen Expansionsachse $(x \approx 5 \mathrm{~mm})$ mit steigendem Abstand zum Düsenausgang. Spektren hinten im Bild entsprechen dabei einer Position nahe der Schlitzdüse. Wie bereits bei der Darstellung der Bandenintegrale gezeigt, weisen auch die Intensitäten an der Schlitzdüsenposition und den beiden auf der untersuchten Spur liegenden Stoßfronten Maxima auf. Zusätzlich ist zu erkennen, dass sich im Bereich der Stoßfronten die Halbwertsbreite der Bande und der Abstand der Maxima der Monomer-P- und R-Zweige zueinander erhöhen. In den Expansionszonen hingegen verringert sich die 


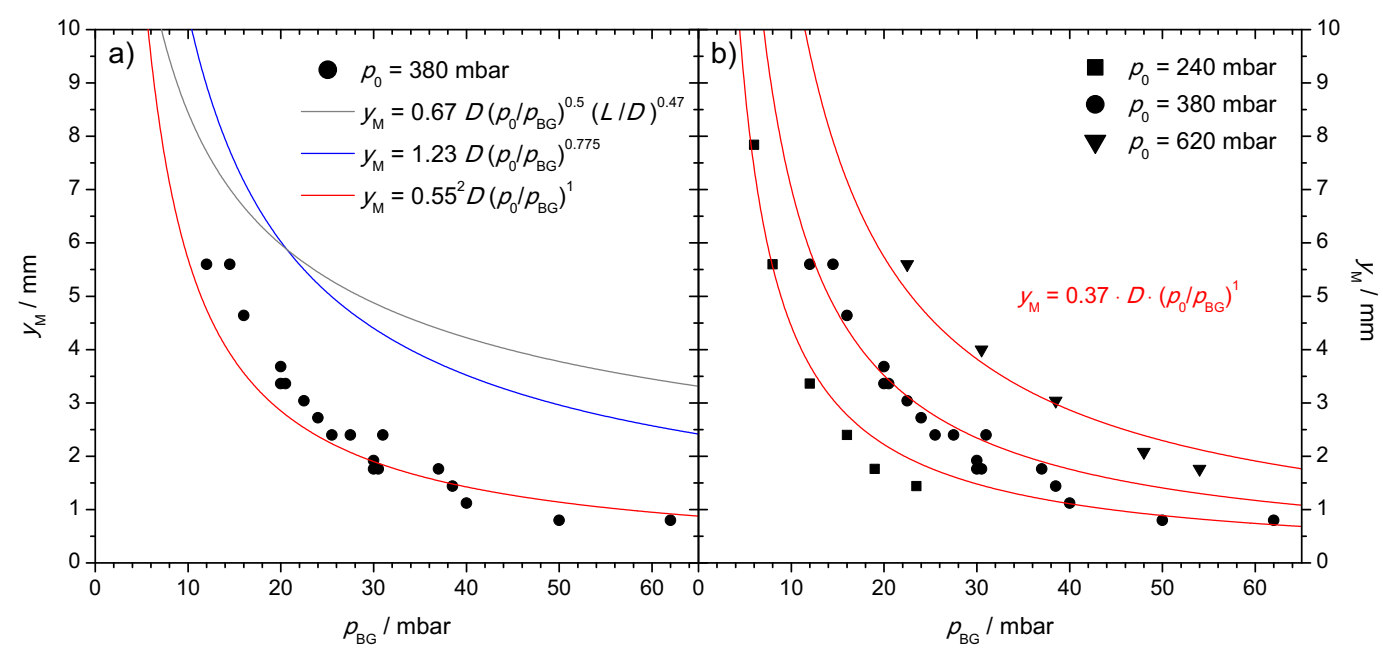

Abbildung 6.6: Position $y_{\mathrm{M}}$ der ersten normalen Stoßfront als Funktion des Hintergrunddrucks $p_{\mathrm{BG}}$ bei Expansionen von $10 \% \mathrm{~N}_{2} \mathrm{O}$ in Helium. a) $p_{0}=380 \mathrm{mbar}$; die drei eingezeichneten Kurven entsprechen den Gleichungen 2.2 (grau), 2.1 (blau) und 6.6 (rot). b) Daten aus Messungen mit verschiedenen Stagnationsdrücken mit empirisch angepasster Fitfunktion angelehnt an Gleichung 6.6.

Bandenbreite. In keinem der Spektren sind Anzeichen für signifikante Clusterbeiträge zu erkennen, was einerseits auf höhere Temperaturen relativ zu den gepulsten Expansionen hinweist und andererseits eine Temperaturuntersuchung gemäß Gleichung 2.11 (S. 16) zulässt.

Zuvor soll jedoch die Abhängigkeit der Position der ersten normalen Stoßfront von den eingesetzten Druckverhältnissen untersucht werden, um die Gültigkeit der in Abschnitt 2.3 vorgestellten Modelle zu überprüfen. Während dort empirisch gefundene Zusammenhänge für die Voraussetzungen (annähernd) unendlich langer Schlitzdüsen (Gleichung 2.1) oder - bei der hier eingesetzten Düsengeometrie - von Abständen der Stoßfront zum Schlitzdüsenausgang von mehr als $8 \mathrm{~mm}$ vorgestellt wurden, die aufgrund der Schlitzdüsenlänge von $10 \mathrm{~mm}$ und der begrenzten untersuchbaren Fläche von $1 \times 1 \mathrm{~cm}^{2}$ bereits formal von den gegebenen experimentellen Bedingungen abweichen, existiert für endlich lange Schlitzdüsen und kleine Druckverhältnisse $\frac{p_{0}}{p_{\mathrm{BG}}}$ eine Vorhersage aus Referenz 25. Diese prognostiziert einen Zusammenhang der Form

$$
y_{\mathrm{M}}=0.55^{2} \cdot D \cdot \frac{p_{0}}{p_{\mathrm{BG}}} .
$$

Die linke Hälfte von Abbildung 6.6 zeigt einen Vergleich der experimentell bestimmten Stoßfrontpositionen $y_{\mathrm{M}}$ kontinuierlicher Expansionen mit $p_{0}=380$ mbar als Funktion des Hintergrunddrucks mit verschiedenen Vorhersagemodellen. Hierbei wurden die Punkte mit dem größten Dichteanstieg zwischen dem Minimum der freien Expansion und dem Maximum der ersten normalen Stoßfront unter Einbeziehung einer Abstandskorrektur zur Berücksichtigung der bei der Bildglättung weggefallenen Pixel 
als Stoßfrontposition verwendet. Messungen mit Hintergrunddrücken unterhalb von 12 mbar wurden nicht mit einbezogen, da die Bestimmung der Stoßfrontpositionen hier aufgrund der langgestreckten Dichteanstiege zwischen Expansions- und Kompressionszone stark fehlerbehaftet war. Schwarze Kreise stellen die einzelnen Messpunkte dar. Die Funktionen $y_{\mathrm{M}}\left(p_{\mathrm{BG}}\right)$ sind durch Linien dargestellt. Die graue Kurve zeigt den empirisch gefundenen Verlauf für große $y_{\mathrm{M}}$ nach Gleichung 2.2, die blaue Kurve den für die unendlich lange Düse gefundenen Zusammenhang (Gl. 2.1) und die rote Kurve die Vorhersage für kleine $y_{\mathrm{M}}$ (Gl. 6.6). Hierbei markiert der Schnittpunkt der roten und grauen Kurve knapp außerhalb des gezeigten Bereichs den theoretischen Übergang zwischen den beiden Modellen aus Referenz 25.

Es zeigt sich, dass die Vorhersage für eine unendlich lange Schlitzdüse (blaue Kurve in Abbildung 6.6 a) zwar den prinzipiellen Verlauf wiedergibt, allerdings zu zu hohen Werten von $y_{\mathrm{M}}$ führt. Diese Abweichungen sind modellbedingt zu erwarten, da die verwendete Schlitzdüse ein Länge/Breite-Verhältnis von nur 20 besitzt. Der Verlauf der grauen Kurve entsprechend Gleichung $2.2^{25}$ wurde empirisch nur für größere Verhältnisse $\frac{p_{0}}{p_{\mathrm{BG}}}$ erhalten, hieraus erklären sich auch die deutlichen Abweichungen zu den dargestellten Messpunkten. Der bislang nicht experimentell belegte, einfach reziproke Verlauf für kleinere Druckverhältnisse gemäß Gleichung 6.6 (rote Kurve) zeigt hingegen eine bemerkenswerte Übereinstimmung mit den Datenpunkten. Auch die Gegenprobe durch eine Fitfunktion mit variablem Exponenten liefert Werte für diesen, die nur leicht über 1 liegen.

Um diese Übereinstimmung auch für weitere Messreihen mit verschiedenen Stagnationsdrücken überprüfen zu können, zeigt der Graph in Abbildung 6.6 b eine Anpassung entsprechend dem Zusammenhang $y_{\mathrm{m}} \propto \frac{p_{0}}{p_{\mathrm{BG}}}$ mit einem empirisch bestimmten Proportionalitätsfaktor von $0.185 \mathrm{~mm}=0.37 \mathrm{D}$, der somit nahe am Literaturfaktor von $0.55^{2} D \approx 0.30 D$ liegt.

$$
y_{\mathrm{M}}=0.37 \cdot D \cdot \frac{p_{0}}{p_{\mathrm{BG}}} .
$$

Es wird eine gute Übereinstimmung mit allen drei durchgeführten Messreihen erhalten, auch wenn die Abweichungen eine bessere Beschreibung durch einen leicht höheren Exponenten erkennen lassen. Differenzen zwischen den experimentellen Daten und der Fitfunktion können auch entstehen, wenn beispielsweise bei der Justierung der Düsenkante auf die Bildgrenze die Düse zu weit aus dem Bild geschoben wird, so dass die aus diesen Messungen erhaltenen Datenpunkte systematisch zu klein sind. Zudem ist die Bestimmung der Stoßfrontposition innerhalb gewisser Grenzen subjektiv und der Anzeigeoffset der verwendeten Druckmessgeräte für die Einstellung der Stagnationsund Hintergrunddrücke kann über den Messtag leicht schwanken.

Neben der Bestätigung der prognostizierten einfach reziproken Abhängigkeit der Stoßfrontposition vom Hintergrunddruck bei kleinen Abständen zur Düse aus Referenz 25 bestätigen die erhaltenen Daten zusätzlich, dass bei den betrachteten Abständen $y<L$ noch kein Übergang des ebenen- in einen achsensymmetrischen Fluss erfolgt, wie er für größere Düsenabstände vorliegt. ${ }^{28}$ 

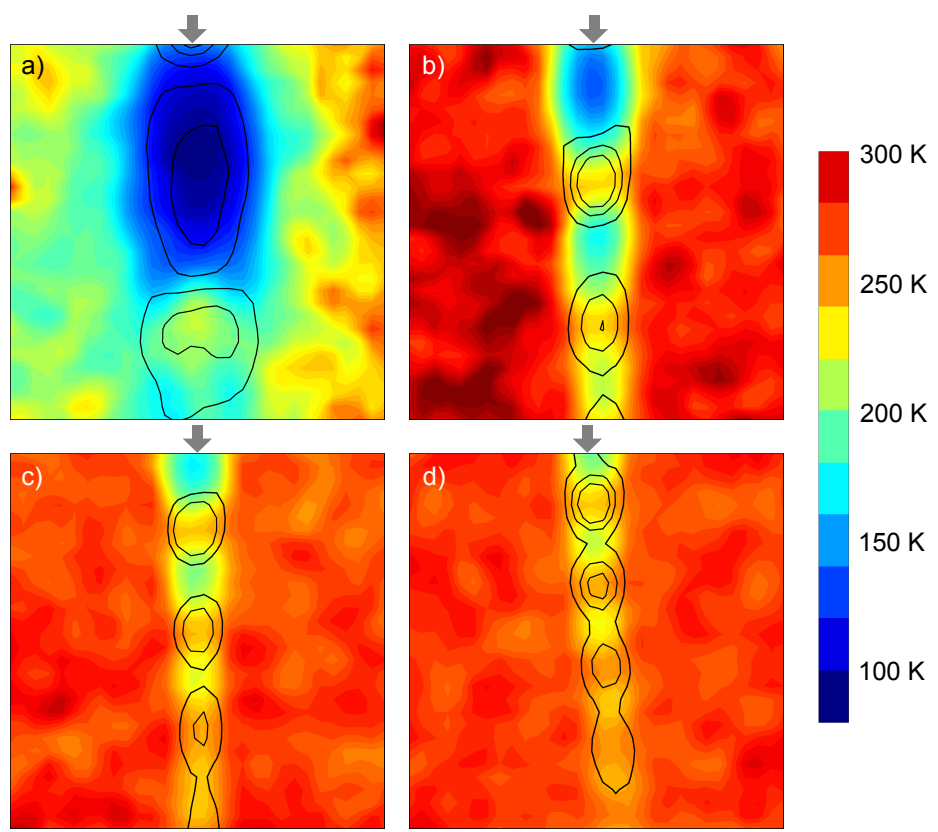

Abbildung 6.7: Temperaturverlauf der Expansionen aus Abbildung 6.4. Die Temperaturen wurden gemäß Gleichung 2.11 unter Zuhilfenahme eines Gasphasenspektrums als 300 K-Referenz bestimmt. Die Skala rechts ermöglicht die Zuordnung von Farbbereichen zu Temperaturen, schwarze Konturlinien deuten den Dichteverlauf an. Mit Ausnahme von Abbildung a) sind nur Dichtemaxima gezeigt. Graue Pfeile markieren die Positionen des Düsenausgangs. a) $p_{\mathrm{BG}}=10 \mathrm{mbar}$. b) $p_{\mathrm{BG}}=20 \mathrm{mbar}$. c) $p_{\mathrm{BG}}=30$ mbar. d) $p_{\mathrm{BG}}=40$ mbar.

\subsubsection{Temperaturverlauf}

Die Spektren in Abbildung 6.5 haben gezeigt, dass aufgrund fehlender Clusterbanden die Anwendung von Gleichung 2.11 zur Bestimmung der Rotationstemperatur aus den Positionen der Maxima von P- und R-Zweig der $\mathrm{N}_{2} \mathrm{O}-\nu_{3}$-Monomerbande möglich sein sollte. Anstelle der Berechnung über die Rotationskonstante von Distickstoffmonoxid wurde ein bei $300 \mathrm{~K}$ aufgenommenes Referenzspektrum mit einer Peakdifferenz von $\Delta \tilde{\nu}_{\mathrm{PR}}^{300 \mathrm{~K}}\left(\nu_{3}\right)=21 \mathrm{~cm}^{-1}$ verwendet. Die erhaltenen Temperaturen ergeben sich somit als

$$
T=\frac{300 \mathrm{~K}}{\left(21 \mathrm{~cm}^{-1}\right)^{2}} \cdot\left(\Delta \tilde{\nu}_{\mathrm{PR}}^{\max }\right)^{2} \approx 0.68 \frac{\mathrm{K}}{\mathrm{cm}^{-2}} \cdot\left(\Delta \tilde{\nu}_{\mathrm{PR}}^{\max }\right)^{2} .
$$

Die so erhaltenen Abschätzungen der Monomer-Rotationstemperatur für die in Abbildung 6.4 gezeigten Expansionen sind in Abbildung 6.7 dargestellt. Die Färbung gibt die erhaltenen Temperaturwerte entsprechend der seitlichen Skala an, während die schwarzen Konturlinien den Dichteverlauf (entsprechend Abb. 6.4) zeigen. Mit Ausnahme der Expansion mit einem Hintergrunddruck von 10 mbar in Abbildung 6.7 a, bei der auch das Dichteminimum in der Ruhezone eingezeichnet ist, zeigen die Konturlinien nur die Lage von Dichtemaxima an. 
Die erhaltenen Temperaturkarten bestätigen eine Temperatur des Hintergrundgases von knapp $300 \mathrm{~K}$, wie sie in durch die Expansion unbeeinflussten Regionen vorliegen sollte. Am Düsenausgang ist das expandierte Gas gegenüber den Stagnationsbedingungen bereits auf einen Wert unterhalb von $200 \mathrm{~K}$ abgekühlt, wobei allerdings zu beachten ist, dass in den geglätteten Spektren der erste Datenpunkt hinter der Düse in etwa dem Wert bei einem mittleren Abstand von $0.5 \mathrm{~mm}$ entspricht. Im weiteren Verlauf der freien Expansionen sinkt der Wert weiter auf bis zu $80 \mathrm{~K}$ bei einem Hintergrunddruck von 10 mbar (Abbildung 6.7 a). Je höher jedoch der Hintergrunddruck ist, desto kleiner ist auch die Zone der freien Expansion, entsprechend ist dort die zur Abkühlung zur Verfügung stehende Zeitdauer geringer und die erreichte Minimaltemperatur höher. In der sich anschließenden Folge abwechselnder Kompression und Reexpansion ist die Korrelation zwischen Dichte- und Temperaturverlauf hervorragend zu erkennen. Dichteanstiege werden von einer Erwärmung begleitet, wobei Temperaturen von bis zu $230 \mathrm{~K}$ in der ersten normalen Stoßfront erreicht werden. Die Reexpansionen hingegen werden von einer Abkühlung auf bis zu $170 \mathrm{~K}$ begleitet, die den in den Dichteverläufen nicht so offensichtlichen Charakter gerichteter Expansionen bestätigen. Je mehr Expansions-Kompressions-Zyklen der Freistrahl durchläuft, desto höher ist die Temperatur der Minima und Maxima, bis sie schließlich, ebenso wie die Dichte, das Niveau des Hintergrundgases erreicht.

Wie bereits beim Dichteverlauf erfolgt, so soll auch für den Temperaturverlauf ein Vergleich mit Vorhersagen aus verschiedenen Modellen vorgenommen werden. Diese Modelle sind nur auf den Temperaturabfall zu Beginn der Expansion anwendbar, daher wird als experimenteller Datensatz die Expansion mit dem geringstem Hintergrunddruck und somit der längsten Abkühlphase (Abbildung 6.7 a) verwendet.

Für die Temperaturvorhersage existieren zum einen die empirischen Formeln ${ }^{25}$ aus Gleichung 2.4, die für die hier betrachteten geringen Abstände der Stoßfront zur Düse $\left(y_{\mathrm{M}}<L\right)$ eine Abhängigkeit von der Stagnationstemperatur $T_{0}$, der Schlitzbreite $D$ und dem Poisson-Koeffizienten $\gamma=\frac{C_{p}}{C_{V}}$ vorhersagen. Zum anderen soll ein Vergleich mit dem Modell einer isentropen Expansion eines idealen Gases vorgenommen werden, in dem die Temperatur für alle Freiheitsgrade identisch und nur an den Dichteverlauf gekoppelt ist ${ }^{24,28,95}$ (Gleichung 2.5).

Abbildung 6.8 zeigt den experimentellen Temperaturverlauf im Zentrum $(x \approx 5 \mathrm{~mm})$ der Expansion von $10 \% \mathrm{~N}_{2} \mathrm{O}$ in Helium mit einem Stagnationsdruck von 380 mbar und einem Hintergrunddruck von 10 mbar aus Abbildung 6.7 a als schwarze Linie. Die Ergebnisse aus dem empirischen Temperaturverlauf gemäß Gleichung 2.4a mit $D=0.5 \mathrm{~mm}$ sind rot eingezeichnet. Für das Modell der isentropen Expansionen wurde das Verhältnis zwischen lokalem und Stagnationsdruck durch das Verhältnis der integrierten $\nu_{3}$ Bandenintensitäten $A$ gewählt. $A_{0}$ wird dabei aus einem Gasphasenspektrum mit bekanntem $\mathrm{N}_{2} \mathrm{O}$-Druck von 4 mbar und Anpassung an den $\mathrm{N}_{2} \mathrm{O}$-Partialdruck im Reservoir bei den Expansionen erhalten. Da der Zusammenhang nach Gleichung 2.5 so nur für achsensymmetrische Expansionen gilt, muss zusätzlich ein (empirischer) Vorfaktor $C$ eingeführt werden ${ }^{28}$, der hier so gewählt wurde, dass für kleine Werte von 


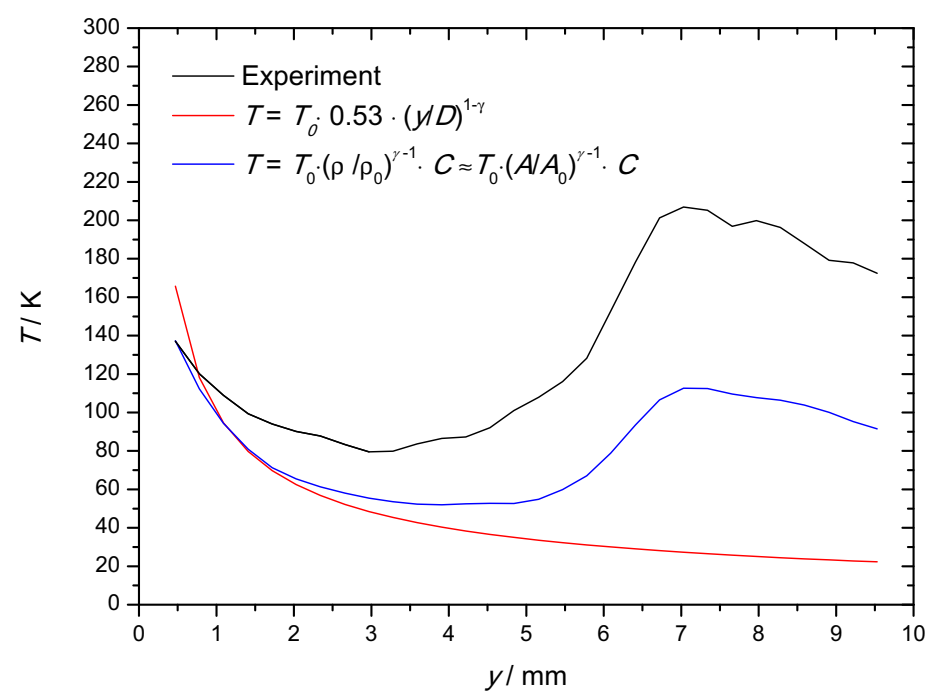

Abbildung 6.8: Vergleich des experimentellen Rotationstemperaturverlaufs mit Modellvorhersagen. Schwarze Linie: Temperaturverlauf einer kontinuierlichen Expansion von $10 \% \mathrm{~N}_{2} \mathrm{O}$ in Helium mit einem Hintergrunddruck von 10 mbar aus Abbildung 6.7 a, $x=5 \mathrm{~mm}$. Rote Linie: Temperatur gemäß empirischer Formel aus Referenz 25 für $y<L$ (Gleichung 2.4a). Blaue Linie: Bestimmung aus der Dichte / den Bandenintegralen unter Annahme der isentropen Expansion eines idealen einatomigen Gases (Gleichung 2.5, modifiziert mit empirischen Vorfaktor). $T_{0}=300 \mathrm{~K}, \gamma=\frac{5}{3}$, $A_{0}\left(10^{3} \ln \frac{I_{0}}{I}\right)=26500 \mathrm{~cm}^{-1}, D=0.5 \mathrm{~mm}, C=3.1$.

$y$ eine Übereinstimmung mit der experimentellen Temperatur erreicht wird. Der aus diesem Ansatz erhaltene Temperaturverlauf ist in Abbildung 6.8 als blaue Linie dargestellt. Obwohl das Verfahren aufgrund des Dichteanstiegs an der normalen Stoßfront in der Lage ist, die damit verbundene Temperaturerhöhung qualitativ wiederzugeben, ist auch hier eine Anwendung aufgrund der Isentropiebedingung nur im Bereich der Abkühlung zu Beginn der Expansion zulässig. Für beide Berechnungen wurde eine Stagnationstemperatur von $T_{0}=300 \mathrm{~K}$ und ein Verhältnis der spezifischen Wärmekapazitäten von $\gamma=\frac{5}{3}$ entsprechend dem eines idealen einatomigen Gases verwendet, da Helium in der Expansion als Überschusskomponente vorliegt.

Beide Verfahren zur Temperaturmodellierung zeigen einen gegenüber den experimentellen Daten deutlich schnelleren Temperaturabfall hinter der Schlitzdüse. Im Falle der empirischen Gleichung $2.4 \mathrm{a}^{25}$ entspricht zwar die Anfangstemperatur in etwa der experimentell gefundenen, im weiteren Verlauf ergibt sich jedoch eine deutliche Diskrepanz. Diese ist auf das in den Chilli-Jet-Expansionen deutlich kleinere Druckverhältnis $\frac{p_{0}}{p_{\mathrm{BG}}}$ zurückzuführen, das bereits zu Abweichungen bei der Bestimmung der Stoßfrontposition (graue Kurve in Abbildung 6.6) geführt hat. Ähnlich große Abweichungen zwischen Experiment und Vorhersage zeigen sich für das Modell der isentropen Expansionen eines idealen Gases, dargestellt als blaue Kurve in Abbildung 6.8, obwohl hier bereits 
der Vorfaktor so angepasst wurde, dass sich für kleine Abstände zur Düse eine Übereinstimmung mit der experimentellen Temperatur ergibt und die Simulation aufgrund der Verwendung der Bandenintegrale als Maß für die Dichte sogar auf den Messdaten beruht. Die zunehmende Differenz zwischen der durch das Modell vorhergesagten Temperatur und den experimentellen Werten kann dabei als Hinweis auf eine eingeschränkte Gültigkeit der Isentropiebedingung und/oder auf den unvollständigen Energietransfer zwischen den Freiheitsgraden von Distickstoffmonoxid und Helium gesehen werden. Gleichzeitig muss jedoch beachtet werden, dass auch die Rotationstemperaturbestimmung aus den ortsaufgelösten Spektren einem groben Modell folgt und somit ebenfalls als fehlerbehaftet anzusehen ist.

\subsection{Zusammenfassung und Ausblick}

Die vorgestellten Untersuchungen von Distickstoffmonoxid als erstem Testsystem haben ergeben, dass die Chilli-Jet-Apparatur für die Untersuchung von Dichte- und Temperaturverläufen sowie der Aggregationsdynamik in Expansionen gut geeignet ist. Die Methode hebt sich von anderen Bildgebungsverfahren durch die Möglichkeit der Korrelation von Expansionsstruktur und chemischer Information bei synchroner Aufnahme des gesamten Probebereichs ab. Im Rahmen dieser Arbeit wurde so durch sukzessive Optimierung des optischen Aufbaus, der Mess- und Auswertemethodiken eine funktionstüchtige Apparatur zur chemischen Bildgebung von Überschallexpansionen mit deutlichen Vorteilen gegenüber Rastermethoden entwickelt.

Trotz der Reduktion des Bild- und pixelübergreifenden Rauschens ist die Einsatzbreite des Chilli-Jets aktuell aufgrund des im Vergleich zu Einzelelementdetektoraufbauten hohen Rauschlevels und des durch das Infrarotobjektiv eingeschränkten untersuchbaren Spektralbereichs auf Systeme mit stark infrarotaktiven Banden zwischen 3000 und $800 \mathrm{~cm}^{-1}$ limitiert. Eine Messung von typischen wasserstoffbrückenbildenden Substanzen wie Wasser und Alkoholen war daher bislang nicht möglich. Neuere Detektorgenerationen weisen aufgrund verbesserter Datenübertragungstechniken ein geringeres Rauschniveau auf, auch die Verwendung eines InSb Focal Plane Array Detektors könnte die Einsatzbreite der Apparatur erweitern. Da die Anschaffungskosten für diese Detektoren jedoch sehr hoch sind, sollte eine Verbesserung der Apparatur zunächst auf anderem Wege angestrebt werden.

Der Einbau eines neuen Objektivs, das auch für Wellenlängen oberhalb von $3000 \mathrm{~cm}^{-1}$ durchlässig ist, sollte Messungen mit konzentrierten Wasser- und Alkohol-TrägergasMischungen ermöglichen. Auch eine Verlängerung des Absorptionswegs durch Einsatz einer längeren Schlitzdüse ist durch die daraus folgende Erhöhung des Signal-zu-Rauschen-Verhältnisses sinnvoll. Eine Abschätzung der maximal verwendbaren Düsenlänge ohne signifikante Einbußen bei der räumlichen Auflösung zeigt Abbildung 6.9. Dargestellt sind die im Justiermodus erhaltenen Bilder geschwärzter Metall-U-Profile unterschiedlicher Länge, die im Fokus des Infrarotlichts möglichst parallel zum Lichtstrahl 


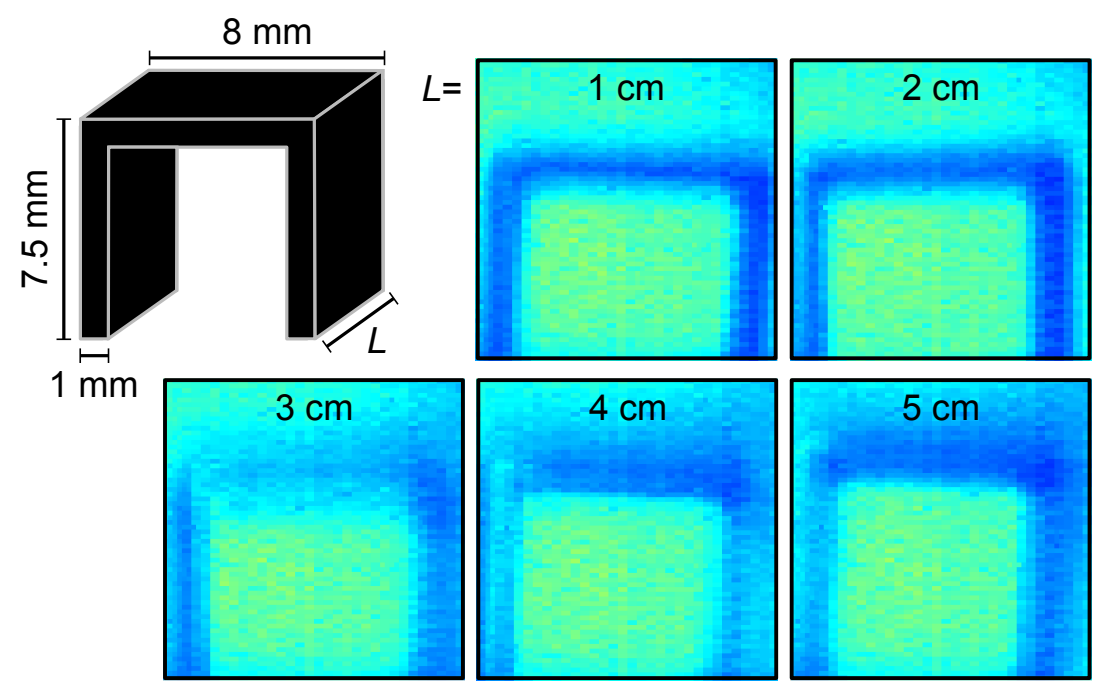

Abbildung 6.9: Abschätzung der maximal einsetzbaren Düsenlänge. Gezeigt sind im Justiermodus aufgenommene Bilder geschwärzter U-Profile der links oben gezeigten Abmessungen im Fokus des Infrarotstrahls. Blau: geringe Lichtintensität; gelb/grün: hohe Lichtintensität.

positioniert wurden. Blaue Bereiche kennzeichnen dabei Orte starker Lichtabschwächung, grüne und gelbe Bereiche repräsentieren hohe Lichtintensitäten. Die Bilder zeigen deutlich, dass bei einem zu langen Probekörper die Unschärfe des abgebildeten Profils stark zunimmt. Bei einer Expansion durch eine Schlitzdüse entsprechender Länge ist somit auch mit massiven Einbußen bei der räumlichen Auflösung zu rechnen. Eine Länge von $1 \mathrm{~cm}$ entspricht der aktuellen Schlitzdüsenlänge. Abbildung 6.9 zeigt deutlich, dass der Kontrast zwischen Probekörper und Umgebung bei Profillängen von 1 und $2 \mathrm{~cm}$ ähnlich ist, ab einer Länge von $3 \mathrm{~cm}$ aber stark abnimmt, so dass eine Verlängerung der Schlitzdüse auf $2 \mathrm{~cm}$ möglich scheint, dieser Wert jedoch nicht überschritten werden sollte. Dies entspricht immerhin einer Verdoppelung des Absorptionswegs, allerdings auch des Gasdurchsatzes, was eine Herausforderung für das Pumpensystem darstellt. Unter Umständen ist bei einer längeren Schlitzdüse daher der Übergang auf den „Single Sided“-Messmodus notwendig, um so die Pulsdauern zu verkürzen.

Wird auf diese Weise das Signal-zu-Rauschen-Verhältnis leicht erhöht, bieten sich auch ohne einen Wechsel des Objektivs neue Messsysteme an. Im Fokus stehen hierbei insbesondere Moleküle mit $\mathrm{C}=\mathrm{O}$-Doppelbindungen, die stark infrarotaktive Streckschwingungen mit Wellenzahlen unterhalb von $3000 \mathrm{~cm}^{-1}$ (typischerweise um $1700 \mathrm{~cm}^{-1}$ ) aufweisen. Neben Kohlenstoffdioxid wären so vor allem Ketone wie Aceton von Interesse, bei denen auch beispielsweise die gemischten Aggregate mit Wasser und der Einfluss der Ausbildung von Wasserstoffbrückenbindungen auf die $\mathrm{C}=\mathrm{O}$-Streckschwingung ortsaufgelöst untersucht werden könnten.

Längerfristige Projekte für die chemische Bildgebung von Überschallexpansionen 
können sich mit der Aufklärung der Aggregationsdynamik von Alkoholen ${ }^{114}$ befassen. Aufgrund der Ortsauflösung könnten so Bildungsmechanismen von Clustern unterschiedlicher Größe untersucht werden. Auch für Systeme mit konkurrierenden Wechselwirkungen (wie Imidazol / Wasser) ${ }^{121}$ oder mehreren Konformeren (z. B. 3,3,3-Trifluorpropanol $)^{60}$ könnte die Bildgebung interessante Aspekte im Bezug auf Umlagerungsprozesse gerade zu frühen Zeitpunkten der Expansion beisteuern. Ebenso kann hier die Betrachtung des Einflusses der periodischen Expansions- und Kompressionszonen aufschlussreich für das Verständnis inter- und intramolekularer Wechselwirkungen sein. 


\section{Zusammenfassung und Ausblick}

Im Rahmen dieser Arbeit wurden mittels Fourier-Transformations-Infrarot-Spektroskopie an Überschall-Schlitzdüsenexpansionen der Einfluss thermischer Anregung auf die Strukturen kleiner Wasseraggregate sowie die bevorzugten Bindungsmotive in Koaggregationsprozessen untersucht. Dies erfolgte mithilfe verschiedener, an die jeweilige Problemstellung angepasster experimenteller Aufbauten.

Für Koexpansionen von Wasser mit schwerflüchtigen Substanzen kam hierbei der bereits bestehende Popcorn-Jet ${ }^{108,109,110}$ zum Einsatz, der über eine heizbare Probenkammer für Komponenten mit geringen Dampfdrücken verfügt. Der Multischlitzdüsenaufbau des Muesli-Jets ${ }^{59,60,258}$ wurde hinsichtlich seiner Expansionseigenschaften charakterisiert und die Fähigkeit zur Generierung warmer Expansionen wurde maximiert. Durch den Entwurf einer alternativen Düsengeometrie ist nun zusätzlich die Erzeugung gemischter Aggregate innerhalb von Überschallexpansionen ohne Vormischung der Komponenten möglich, wie sie für eine Koexpansion reaktiver Mischungen vonnöten ist. Der im Chilli-Jet realisierte neuartige Ansatz der chemischen Bildgebung von Überschallexpansionen durch Einsatz eines Focal-Plane-Array-Detektors ermöglicht nun auch die Aufnahme ortsaufgelöster FTIR-Spektren. Diese liefern nicht nur Einblicke in die Aggregationsdynamik innerhalb der Expansionen, sondern ermöglichen auch Untersuchungen der Dichte- und Temperaturprofile in Überschallstrahlen.

Die Erzeugung warmer Wassercluster in Muesli-Jet-Expansionen liefert ein Bindeglied zwischen den bekannten, kalten Aggregaten, die in typischen Schlitzdüsenexpansionen erzeugt werden ${ }^{107}$, und den warmen Clustern in der Gasphase, deren Nachweis sich problematisch gestaltet und für das Dimer erst kürzlich mittels Rotationsspektroskopie und relativ hohen Wasser-Konzentrationen ${ }^{6}$ gelang. Vergleiche der Spektren warmer Expansionen mit simulierten Bandenformen weisen auf Monomer-Rotationstemperaturen von bis zu $160 \mathrm{~K}$ hin. Es zeigt sich, dass die thermische Anregung intermolekularer Moden unterschiedliche Auswirkungen auf die Strukturen kleiner Wasseraggregate hat. Für das stabile, cyclische Tetramer kann bei gegenüber typischen Expansionsbedingungen erhöhten Temperaturen eine deutliche Blauverschiebung der intensiven O-H-Streckschwingungsbande im Spektrum beobachtet werden. Diese spricht für eine Schwächung der Wasserstoffbrückenbindungen und die daraus folgende gleichmäßige Aufweitung des Ringaggregats. Im Gegensatz hierzu steht das Verhalten des gespannten cyclischen Trimers in warmen Expansionen, beim dem die intensivste O-HStreckschwingungsbande gegenüber kälteren Bedingungen praktisch keine Wellenzahlverschiebung erfährt, jedoch deutlich an Intensität verliert, während zusätzliche Bandenintensität bei höheren Wellenzahlen auftritt. Dieses Verhalten ist mit einer Ring- 
öffnung bei Erwärmung und der daraus folgenden Bildung flexibler, kettenförmiger Trimere zu erklären, die bislang vor allem als Bestandteil überkritischen Wassers angenommen wurden. ${ }^{176}$ Über das Strukturverhalten von Wasser-Dimeren kann aufgrund einer wahrscheinlichen Bandenüberlagerung keine verlässliche Aussage getroffen werden. Aufgrund der geringen Stärke der intermolekularen Wechselwirkung ${ }^{159}$ ist jedoch von einer eher geringen Dimerkonzentration auszugehen.

Die Technik der Multischlitzdüsenexpansionen ermöglicht mit einer entsprechenden Düsengeometrie auch die synchrone Expansion und Mischaggregatbildung zweier getrennt zugeführter Komponenten, bei denen eine Vormischung zu unerwünschten Reaktionen führen würde. Ein Beispiel hierfür stellen $\mathrm{H}_{2} \mathrm{O}$ und $\mathrm{D}_{2} \mathrm{O}$ dar, bei deren Mischung eine Bildung von HDO durch Isotopenaustausch erfolgt. Dies führt zu einer deutlich höheren Anzahl möglicher Isotopenkombinationen für die gebildeten Cluster und somit auch zu stärkeren Bandenüberlappungen. Da die Analyse der Schwingungsspektren von Wasser aufgrund der komplexen Rotationsstruktur und der Kopplung der Normalmoden generell erschwert ist, wurde zusätzlich das Modellsystem tert-Butylalkohol+Methanol bzw. + Deuteromethanol betrachtet. Dies hat gezeigt, dass bei einer parallelen Anordnung der Schlitzdüsen keine Bildung von Mischaggregaten nachweisbar ist. Mit einer paarweise konvergierenden Anordnung der Schlitze ist eine Erzeugung gemischter Aggregate in der Expansion möglich, wobei Isotopenaustauschreaktionen hingegen selbst bei größeren Düsenabständen unterdrückt bleiben. Eine Unterdrückung des Isotopenaustauschs kann auch für synchrone Expansionen von $\mathrm{H}_{2} \mathrm{O}$ und $\mathrm{D}_{2} \mathrm{O}$ bestätigt werden, es werden keine signifikanten Mengen an HDO gebildet. Entsprechend reduziert sich auch die Anzahl möglicher gebildeter Isotopologe und Isotopomere der gemischten Cluster. Da Wasser bevorzugt große, netzwerkartige Aggregate bildet ${ }^{113}$, konnte aufgrund von Bandenüberlappungen eine eindeutige Zuordnung von $\mathrm{H}_{2} \mathrm{O}-\mathrm{D}_{2} \mathrm{O}$ Mischclusterbanden bislang nicht erfolgen. Für zukünftige Messungen mit geringeren Konzentrationen haben Rechnungen das gemischte Trimer $\left(\mathrm{H}_{2} \mathrm{O}\right)_{1}\left(\mathrm{D}_{2} \mathrm{O}\right)_{2}$ als aussichtsreichstes Aggregat für die Bandenidentifikation ergeben. Zusätzlich kann sich das Modellsystem Methanol/Methanol-D1 als hilfreich für Bandenzuordnungen sowie die Analyse von Kopplungsmustern erweisen.

Neben den reinen Wasser-Clustern sind auch gemischte Aggregate von Bedeutung. Die jeweils zwei Wasserstoffbrückendonor- und -akzeptorfunktionalitäten pro Molekül bewirken nicht nur das flüssige Vorliegen von Wasser bei Standardbedingungen. Auf ihnen basieren auch die teilweise für enzymatische Katalyseprozesse essentiellen Bindungen zwischen Aminosäureresten und einzelnen Wassermolekülen, wie sie beispielsweise zwischen Histidin und Wasser in der katalytischen Triade innerhalb von Serinproteasen auftreten. ${ }^{7}$ Die Imidazolylgruppe in Histidin kann - wie Wasser - in Wasserstoffbrückenbindungen sowohl den Donor als auch den Akzeptor darstellen, so dass zwei verschiedene Bindungsmotive (O-H. ․ N, N-H ․ O ) möglich sind. Die Untersuchungen am Popcorn-Jet haben dabei ergeben, dass im Modellsystem Imidazol-Wasser die Orientierung des Wassermoleküls als Donor bevorzugt ist, wobei das am Imidazol-N3-Stickstoff lokalisierte, in der Molekülebene liegende freie Elektronenpaar als Akzeptor fungiert. ${ }^{121}$ 
Das makroskopisch basische Verhalten von Imidazol in wässriger Lösung zeigt sich folglich bereits auf molekularer Ebene. Diese Präferenz in den Überschallexpansionen ist mechanistisch interessant und beruht wahrscheinlich auf Wasseraustauschprozessen in den frühen, stoßreichen Stadien der Expansion. Wird Methanol als Solvens verwendet, besteht diese Präferenz weiter, ist jedoch schwächer ausgeprägt. Der Einsatz von phenylsubstituiertem Imidazol zur Nachbildung der in Histidin auftretenden Tautomerie zeigt eine nur eingeschränkte Übertragbarkeit, stellt allerdings ein interessantes System für die Untersuchung des Einflusses von $\mathrm{C}-\mathrm{H} \cdots \mathrm{O}$ - und $\mathrm{O}-\mathrm{H} \cdots \pi$-Bindungen als konkurrierende Wechselwirkungen zu den typischen $\mathrm{O}-\mathrm{H} \cdots \mathrm{N}$ und $\mathrm{N}-\mathrm{H} \cdots \mathrm{O}-$ Wasserstoffbrückenbindungen dar.

Ein Nachteil der bislang vorgestellten Versuchsanordnungen liegt darin, dass aufgrund von hohen Infrarotstrahldurchmessern ein großer Bereich der Expansionszone gemittelt wird und so dynamische Prozesse wie Aggregatbildungen und Umlagerungen nur sehr eingeschränkt untersucht werden können. Eine ortsaufösende, synchrone, schwingungsspektroskopische Untersuchung von Aggregationsprozessen wird durch die Kombination mit Focal Plane Array (FPA) Detektoren ermöglicht. Da diese gegenüber entsprechenden Einzelelementdetektoren ein deutlich geringeres Signal-zu-Rauschen Verhältnis aufweisen, wurde aufgrund seiner hohen Bandenstärken als erstes Testsystem Lachgas $\left(\mathrm{N}_{2} \mathrm{O}\right)$ gewählt. Die durchgeführten Messungen haben gezeigt, dass die chemische Bildgebung mit dem Chilli-Jet fähig ist, den Dichte- und Temperaturverlauf in Expansionen wiederzugeben. ${ }^{88}$ Dabei zeigte sich hinter der ersten normalen Stoßfront ein periodisches Muster aus Reexpansions- und Kompressionszonen mit entsprechenden Temperaturminima und -maxima, dessen Einfluss auf Aggregatstrukturen und Konformerenverhältnisse Gegenstand folgender Untersuchungen sein wird. Aus den Dichteuntersuchungen konnte zusätzlich ein prognostizierter Zusammenhang ${ }^{25}$ zwischen der Position der ersten normalen Stoßfront und dem Druckverhältnis $\frac{p_{0}}{p_{\mathrm{BG}}}$ für kleine Stagnations- und hohe Hintergrunddrücke experimentell verifiziert werden. Außerdem können Aggregationsprozesse ortsaufgelöst visualisiert werden, so dass Bildungs-, Zerfalls- und Umlagerungsmechanismen zugänglich werden.

Neben den erhaltenen Erkenntnissen zu den Strukturen warmer und gemischter Wasseraggregate wurde im Rahmen dieser Arbeit auch die Anwendungsbreite der FTIRSpektroskopie an Überschall-Schlitzdüsenexpansionen erweitert. Der Muesli-Jet ermöglicht die Untersuchung von thermisch angeregten Clustern sowie von Heteroaggregaten reaktiver Komponenten durch synchrone Expansion bei getrennter Zufuhr. Der ChilliJet bietet Zugang zur chemischen Bildgebung von Überschallexpansionen und wird in der Zukunft weiter optimiert werden, so dass auch schwächer absorbierende Substanzen betrachtet werden können. Ein Fernziel stellt die ortsaufgelöste Infrarotspektroskopie von Multischlitzdüsenexpansionen, also eine Kombination beider Apparaturen dar. Diese würde Untersuchungen der Dynamik von Koaggregationsprozessen ermöglichen und so unser Verständnis intermolekularer Wechselwirkungen erweitern. 


\section{A Anhang}

\section{A.1 Publikationen}

Im Rahmen dieser Arbeit sind folgende Publikationen entstanden:

- J. Zischang, J. J. Lee, M. A. Suhm, Where does the first water molecule go in imidazole?, J. Chem. Phys. 2011, 135, 061102.

- J. Zischang, M. A. Suhm, Infrared absorption imaging of 2D supersonic jet expansions: Free expansion, cluster formation, and shock wave patterns, J. Chem. Phys. 2013, 139, 024201.

- J. Zischang, M. A. Suhm, The $\mathrm{OH}$ stretching spectrum of warm water clusters J. Chem. Phys. 2014, 140, 064312.

\section{A.2 Verwendete Chemikalien}

\begin{tabular}{lclcl}
\hline Substanz & CAS-Nr. & Hersteller & Reinheit & Indent.-Nr. \\
\hline \hline Wasser & $7732-18-5$ & Acros & HPLC grade & A0260415 \\
Wasser & $7732-18-5$ & Carl Roth & p.a. & 049101881 \\
Wasser & $7732-18-5$ & - & demin. & - \\
Deuteriumoxid & $7789-20-0$ & euriso-top & $99.90 \%$ D & X3191 \\
Wasser- ${ }^{18}$ O & $14314-42-2$ & & $98.37 \%{ }^{18} \mathrm{O}$ & \\
Methanol & $67-56-1$ & Merck & $>99.9 \%$ & K37186309 \\
Methanol & $67-56-1$ & Sigma-Aldrich & p.a. & SZBA0490 \\
Methanol-D1 & $1455-13-6$ & euriso-top & $99.00 \%$ & X1071 \\
Methanol-D1 & $1455-13-6$ & euriso-top & $99.80 \%$ & U0181 \\
tert-Butylalkohol & $75-65-0$ & Carl Roth & $\geq 99 \%$ & 012180819 \\
Imidazol & $288-32-4$ & Alfa Aesar & $99 \%$ & $91935636 \mathrm{WO}$ \\
Imidazol & $288-32-4$ & ABCR & $99 \%$ & 1115039 \\
4-Phenylimidazol & $670-95-1$ & ABCR & $98 \%$ & 1123613 \\
4-Phenylimidazol & $670-95-1$ & Alfa Aesar & $98 \%$ & 10132469 \\
Lachgas & $10024-97-2$ & Linde & 4.5 & \\
Chlorwasserstoff & $7647-01-0$ & Messer & 2.8 & \\
Helium & $7440-59-7$ & Linde & 4.6 & \\
Helium & $7440-59-7$ & Air Liquide & 4.6 & \\
Molekularsieb 3 Å & & Carl Roth & & 369103515 \\
\hline
\end{tabular}




\section{A.3 Spektrenverzeichnis}

\begin{tabular}{|c|c|c|c|}
\hline Abb. & Datum & Kennung.Scanzahl & Parameterblock \\
\hline $2.1 \mathrm{~b}$ & 130131 & e. e.50, h.50 & 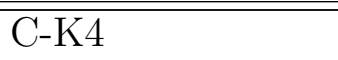 \\
\hline $2.1 \mathrm{c}$ & 130131 & d. 50, f. .50 & $\mathrm{C}-\mathrm{K} 4$ \\
\hline $3.4 \mathrm{a}$ & 130514 & b. 50, c. $75, \mathrm{~d} .75$ & M-I \\
\hline $3.4 \mathrm{~b}$ & 130514 & h.100 & M-I \\
\hline $3.4 \mathrm{c}$ & 130514 & f.100, g.100 & M-I \\
\hline $3.4 \mathrm{~d}$ & 130523 & c. 100, d.100 & M-I \\
\hline $3.4 \mathrm{e}$ & 130524 & e. 50, f. .50, g. 50 & M-I \\
\hline $3.5 \mathrm{~b}$ & 130502 & e. 40 & M-MI \\
\hline $3.5 \mathrm{c}$ & 130731 & b.50, d.100, e.100, f.100 & $\mathrm{M}-\mathrm{W} 1 / 20$ \\
\hline $3.5 \mathrm{~d}$ & 130802 & c. 100, d. 100, e. 50, f.100 & M-W1/20 \\
\hline $3.6 \mathrm{a}$ & 130506 & d. 50 & $\mathrm{M}-\mathrm{I}$ \\
\hline $3.6 \mathrm{~b}$ & 130806 & h.100, i.100 & M-I \\
\hline $3.7 \mathrm{a}$ & 131001 & c. 50 & M-MI \\
\hline $3.7 \mathrm{~b}$ & 131011 & b. 50, c. 50, d. 50, e. 50, f.50 & M-I \\
\hline \multirow[t]{2}{*}{$3.7 \mathrm{c}$} & 131009 & f. 50, g. 50, h. 50, i. 50 & M-I \\
\hline & 131011 & a. 50 & M-I \\
\hline \multirow[t]{2}{*}{$3.7 \mathrm{~d}$} & 131011 & g. 50 & M-I \\
\hline & 131015 & a. 50, b. 50, d. 50, f. 50 & M-I \\
\hline $3.11 \mathrm{a}$ & 131023 & c. 100 & $\mathrm{C}-\mathrm{K} 2$ \\
\hline $3.11 \mathrm{~b}$ & 101018 & 10.32 & $\mathrm{C}-\mathrm{K} 2$ \\
\hline 3.12 & 121008 & test_a. 50 & $\mathrm{C}-\mathrm{K} 2$ \\
\hline 3.13 & 130131 & e. 50, h. .50 & $\mathrm{C}-\mathrm{K} 4$ \\
\hline 3.14 & 121108 & c. 1, a. .50 & $\mathrm{C}-\mathrm{K} 2$ \\
\hline $4.1 \mathrm{a}$ & 130514 & b. 50, c. 75, d. 75 & M-I \\
\hline $4.1 \mathrm{~b}$ & 130418 & f. .50, g. 100 , h. 100 , i. 50 & M-MI \\
\hline \multirow[t]{2}{*}{$4.1 \mathrm{c}$} & 130425 & f. 10 , g. 50 , h. 50 , i. 150 & M-MI \\
\hline & 130506 & d. 50 & M-I \\
\hline \multirow[t]{2}{*}{$4.1 \mathrm{~d}$} & 130429 & g.100 & M-MI \\
\hline & 130506 & e. 50 & M-I \\
\hline $4.1 \mathrm{e}$ & 130508 & c. 50 & M-I \\
\hline $4.1 \mathrm{f}$ & 130806 & b.50, c.50, j.100 & M-I \\
\hline $4.1 \mathrm{~g}$ & 130806 & d.100, k.100 & M-I \\
\hline $4.1 \mathrm{~h}$ & 130806 & h.100, i.100 & M-I \\
\hline $4.2 \mathrm{a}$ & 130806 & b. 50, c. 50, j.100 & M-I \\
\hline $4.2 \mathrm{~b}$ & 130808 & e.100 & M-I \\
\hline $4.2 \mathrm{c}$ & 130806 & d.100, k.100 & M-I \\
\hline \multirow[t]{2}{*}{$4.2 \mathrm{e}$} & 130808 & g. 100 , h. 100 , i.100 & M-I \\
\hline & 130813 & c. 100, d. 100 & M-I \\
\hline
\end{tabular}




\begin{tabular}{|c|c|c|c|}
\hline \multicolumn{4}{|c|}{ Fortsetzung Spektrenverzeichnis } \\
\hline Abb. & Datum & Kennung.Scanzahl & Parameterblock \\
\hline & 130814 & h.50 & M-I \\
\hline \multirow[t]{2}{*}{$4.2 \mathrm{f}$} & 130813 & e.50, f. 100 & M-I \\
\hline & 130814 & g. 50 & M-I \\
\hline $4.2 \mathrm{~g}$ & 130814 & d.10, e.100, f.100 & M-I \\
\hline $4.3 \mathrm{~b}$ & 130731 & b.50, d.100, e.100, f.100 & $\mathrm{M}-\mathrm{W} 1 / 20$ \\
\hline $4.3 \mathrm{c}$ & 130620 & h. 50, i. .50 & M-W05/20 \\
\hline \multirow[t]{3}{*}{$4.3 \mathrm{~d}$} & 130725 & j. $600, \mathrm{k} .600$ & M-W1/10 \\
\hline & 130726 & d. 343, e. $.400, f .400$ & $\mathrm{M}-\mathrm{W} 1 / 10$ \\
\hline & 130729 & c. 100 , d. 400 , e. 800 , g. 600 , h. 800 & $\mathrm{M}-\mathrm{W} 1 / 10$ \\
\hline \multirow[t]{2}{*}{$4.3 \mathrm{e}$} & 130725 & o.40, p.800 & $\mathrm{M}-\mathrm{W} 1 / 20$ \\
\hline & 130726 & b. 100 & $\mathrm{M}-\mathrm{W} 1 / 20$ \\
\hline 4.5 & 130704 & c. $50-\mathrm{s} .50$ & \\
\hline $4.6 \mathrm{a}, \mathrm{b}, \mathrm{c}, \mathrm{e}$ & $(=\mathrm{Abb}$ & 4.3 a,b,c,e) & \\
\hline $4.7 \mathrm{a}-\mathrm{e}$ & $(=\mathrm{Abb}$ & $4.3 \mathrm{a}-\mathrm{e})$ & \\
\hline $4.9 \mathrm{a}, \mathrm{d}, \mathrm{e}$ & $(=\mathrm{Abb}$ & $4.3 \mathrm{a}, \mathrm{d}, \mathrm{e})$ & \\
\hline 4.10 c) a,b,c,e & $(=\mathrm{Abb}$ & 4.3 a,b,c,e) & \\
\hline $4.11 \mathrm{a}$ & 131022 & c. 50, d. 50, e. 50, f. 50, h. 50 & M-I \\
\hline \multirow[t]{2}{*}{$4.11 \mathrm{~b}$} & 131009 & d.50, f.50, g. 50, h. .50 , i. .50 & M-I, M-M (d) \\
\hline & 131011 & a. 50 & M-I \\
\hline \multirow[t]{2}{*}{$4.11 \mathrm{c}$} & 130911 & f. 50, g. 50, h. 50 & M-MI \\
\hline & 130924 & d. 50, e. 50, f. 50 & M-MI \\
\hline $4.11 \mathrm{~d}$ & 131016 & c. 50, d. 50, e. 50, f. 50, g. 50 & M-MI \\
\hline $4.12 \mathrm{a}-\mathrm{c}$ & $(=\mathrm{Abb}$ & $4.11 \mathrm{~b}-\mathrm{d})$ & \\
\hline $4.13 \mathrm{a}, \mathrm{b}, \mathrm{c}$ & $(=\mathrm{Abb}$ & $4.11 \mathrm{a}, \mathrm{c}, \mathrm{d})$ & \\
\hline $4.14 \mathrm{a}$ & 131011 & b.50, c. 50, d. .50, e. 50, f.50 & M-I \\
\hline $4.14 \mathrm{~b}$ & $(=\mathrm{Abb}$ & $4.11 \mathrm{~b})$ & \\
\hline \multirow[t]{2}{*}{$4.14 \mathrm{c}$} & 131011 & g. 50 & M-I \\
\hline & 131015 & a. 50, b. 50, d. 50, f. 50 & M-I \\
\hline \multirow[t]{2}{*}{$4.14 \mathrm{~d}$} & 130910 & f. .50, g. 50, h. 50 & M-MI \\
\hline & 130911 & c. 50, d. .50, e. 50 & M-MI \\
\hline $4.14 \mathrm{e}$ & $(=\mathrm{Abb}$ & 4.11 c) & \\
\hline $4.14 \mathrm{f}$ & 131002 & c. 50, d. 50, e. 50, f. 50, g. 50 & M-I \\
\hline 4.15 & $(=\mathrm{Abb}$ & $4.11 \mathrm{c})$ & \\
\hline $5.2 \mathrm{a}$ & 090825 & j. $50, \mathrm{k} .50,1.50, \mathrm{~m} .50, \mathrm{n} .50$, t. 50 & INSB35DSFR \\
\hline \multirow[t]{2}{*}{$5.2 \mathrm{~b}, \mathrm{c}$} & 091014 & d. .50, e. 50, f. .50 & INSB4DSFR \\
\hline & 091015 & 1.50, m.50, n.50 & INSB4DSFR \\
\hline $5.4 \mathrm{a}$ & $(=\mathrm{Abb}$ & $5.2 \mathrm{a})$ & \\
\hline $5.4 \mathrm{~b}$ & 091202 & i. .50, j.50, 1.50, m.50, n.50, o.50 & INSB4DSFR \\
\hline $5.4 \mathrm{c}$ & 100105 & f. .50, g. 50, h. 50, i. 50, j. 50, k. 50 & INSB35DSFR \\
\hline
\end{tabular}




\begin{tabular}{|c|c|c|c|}
\hline \multicolumn{4}{|c|}{ Fortsetzung Spektrenverzeichnis } \\
\hline Abb. & Datum & Kennung.Scanzahl & Parameterblock \\
\hline $5.4 \mathrm{e}$ & \multicolumn{3}{|c|}{$(=$ Abb. $5.2 \mathrm{a})$} \\
\hline $5.4 \mathrm{f}$ & 091027 & d.50, e.50, f.50, g.50, h.50, i.50 & INSB4DSFR \\
\hline $5.4 \mathrm{~g}$ & 091026 & f. .50, g. 50, h. 50, i. .50, j. .50, k. 50 & INSB4DSFR \\
\hline $5.4 \mathrm{~h}$ & 110609 & e.50, f.50, g.50, h.50, i.50, j.50 & INSB4DSFR \\
\hline $5.6 \mathrm{a}$ & 110629 & e. .50, f. 50, g. .50, h. 50, i. 50, j. 50, k. 50 & INSB35DSFR \\
\hline $5.6 \mathrm{~b}$ & \multicolumn{3}{|c|}{$(=\mathrm{Abb} .5 .2 \mathrm{a})$} \\
\hline $5.6 \mathrm{c}$ & 110630 & f. .50, g. 50 , h. 50 , i. 50, j. 50, k. 50 & INSB35DSFR \\
\hline \multirow[t]{2}{*}{$6.1 \mathrm{a}$} & 121008 & g.47, h.48; & C-P2 \\
\hline & 130206 & c. 100 & C-P2 \\
\hline $6.1 \mathrm{~b}$ & 121002 & h. 32, i. 44 & C-P2 \\
\hline \multirow[t]{2}{*}{6.2 lo } & 121008 & g. 47, h. 48 & $\mathrm{C}-\mathrm{P} 2$ \\
\hline & 130206 & c. 100 & $\mathrm{C}-\mathrm{P} 2$ \\
\hline \multirow[t]{2}{*}{6.2 ro } & 121008 & c. 48, d. 50 & C-P2 \\
\hline & 130206 & d. 17 & $\mathrm{C}-\mathrm{P} 2$ \\
\hline $6.2 \mathrm{lu}$ & 121002 & h. 32, i. 44 & C-P2 \\
\hline $6.2 \mathrm{ru}$ & 120927 & b. 40, c. 42, d. 42, e. 45 & C-P2 \\
\hline $6.3 \mathrm{o}$ & \multicolumn{3}{|c|}{$(=\mathrm{Abb} .6 .2 \mathrm{lo})$} \\
\hline $6.3 \mathrm{u}$ & \multicolumn{3}{|c|}{$(=$ Abb. $6.2 \mathrm{lu})$} \\
\hline $6.4 \mathrm{a}$ & 130131 & e. 50, h. 50 & $\mathrm{C}-\mathrm{K} 4$ \\
\hline $6.4 \mathrm{~b}$ & 130131 & b. $50 \mathrm{i} .50$ & $\mathrm{C}-\mathrm{K} 4$ \\
\hline $6.4 \mathrm{c}$ & 130131 & $\mathrm{~d} .50 \mathrm{f} .50$ & $\mathrm{C}-\mathrm{K} 4$ \\
\hline $6.4 \mathrm{~d}$ & 130131 & c. 50, g. 50 & $\mathrm{C}-\mathrm{K} 4$ \\
\hline 6.5 & $(=\mathrm{Abb}$. & $5.4 \mathrm{~b})$ & \\
\hline \multirow[t]{4}{*}{$6.6380 \mathrm{mbar}$} & 120524 & a. $50-$ u. 50 & $\mathrm{C}-\mathrm{K} 4$ \\
\hline & 120604 & b.50-f.50, h.50--1.50 & $\mathrm{C}-\mathrm{K} 4$ \\
\hline & 120611 & a. $.50-$ g. .50, i. $.50-1.50$ & $\mathrm{C}-\mathrm{K} 4$ \\
\hline & 130131 & b.50-d.50, f.50, g.50, i.50 & C-K4 \\
\hline \multirow[t]{2}{*}{$6.6620 \mathrm{mbar}$} & 120524 & y.50-z2.50 & $\mathrm{C}-\mathrm{K} 4$ \\
\hline & 130131 & p. 25 & $\mathrm{C}-\mathrm{K} 4$ \\
\hline $6.6240 \mathrm{mbar}$ & 130131 & j.25-o. 25 & $\mathrm{C}-\mathrm{K} 4$ \\
\hline $6.7 \mathrm{a}-\mathrm{d}$ & $(=\mathrm{Abb}$. & $6.4 \mathrm{a}-\mathrm{d})$ & \\
\hline \multirow[t]{2}{*}{6.8} & 130131 & e. 50, h. .50 & $\mathrm{C}-\mathrm{K} 4$ \\
\hline & 120524 & a. $50-\mathrm{e} .50$ & $\mathrm{C}-\mathrm{K} 4$ \\
\hline $6.91 \mathrm{~cm}$ & 131023 & b.100 & C-K2 \\
\hline $6.92 \mathrm{~cm}$ & 131023 & d.100 & $\mathrm{C}-\mathrm{K} 2$ \\
\hline $6.93 \mathrm{~cm}$ & 131023 & f. 100 & $\mathrm{C}-\mathrm{K} 2$ \\
\hline $6.94 \mathrm{~cm}$ & 131023 & h.100 & $\mathrm{C}-\mathrm{K} 2$ \\
\hline $6.95 \mathrm{~cm}$ & 131023 & j.100 & C-K2 \\
\hline
\end{tabular}




\section{A.4 Detaillierte Ergebnisse der quantenchemischen Rechnungen}

\begin{tabular}{|c|c|c|c|c|c|c|c|c|}
\hline \multicolumn{9}{|c|}{ Zu: Tabelle 4.1, B3LYP/6-311++G** und MP2/aug-cc-pVDZ } \\
\hline \multirow{2}{*}{ Aggregat } & \multicolumn{2}{|c|}{$\tilde{\nu} / \mathrm{cm}^{-1}$} & \multicolumn{2}{|c|}{$I / \mathrm{km} \mathrm{mol}^{-1}$} & \multicolumn{2}{|c|}{ Skalierungsfaktor } & \multicolumn{2}{|c|}{$D_{e} / \mathrm{kJ} \mathrm{mol}^{-1}$} \\
\hline & B3LYP & MP2 & B3LYP & MP2 & B3LYP & MP2 & B3LYP & MP2 \\
\hline $\mathrm{MeOH}$ & 3847 & 3841 & 30 & 34 & 0.958 & 0.960 & & \\
\hline$(\mathrm{MeOH})_{2}$ & 3690 & 3675 & 508 & 483 & 0.958 & 0.960 & 24.4 & 26.8 \\
\hline MeOD & 2801 & 2797 & 22 & 24 & 0.970 & 0.972 & & \\
\hline$(\mathrm{MeOD})_{2}$ & 2689 & 2677 & 291 & 274 & 0.970 & 0.972 & 24.4 & 26.8 \\
\hline$t \mathrm{BuOH}$ & 3817 & 3793 & 14 & 17 & 0.954 & 0.961 & & \\
\hline$(t \mathrm{BuOH})_{2}$ & 3658 & 3599 & 465 & 484 & 0.954 & 0.961 & 21.6 & 40.9 \\
\hline$t \mathrm{BuOD}$ & 2780 & 2761 & 10 & 12 & 0.967 & 0.974 & & \\
\hline$(t \mathrm{BuOD})_{2}$ & 2667 & 2622 & 257 & 266 & 0.967 & 0.974 & 21.6 & 40.9 \\
\hline $\mathrm{MeOH}-t \mathrm{BuOH}$ & 3666 & 3635 & 573 & 512 & 0.958 & 0.960 & 25.0 & 34.8 \\
\hline$t \mathrm{BuOH}-\mathrm{MeOH}$ & 3678 & 3644 & 414 & 390 & 0.954 & 0.961 & 21.8 & 31.3 \\
\hline $\mathrm{MeOD}-t \mathrm{BuOH}$ & 2671 & 2648 & 327 & 290 & 0.970 & 0.972 & 25.0 & 34.8 \\
\hline$t \mathrm{BuOH}-\mathrm{MeOD}$ & 3680 & 3644 & 412 & 385 & 0.954 & 0.961 & 21.8 & 31.3 \\
\hline
\end{tabular}

\begin{tabular}{|c|c|c|c|c|c|c|}
\hline \multicolumn{7}{|c|}{ Zu: Abbildung 4.9, MP2/aug-cc-pVTZ } \\
\hline \multirow[b]{2}{*}{ Aggregat } & \multicolumn{3}{|c|}{ fopt/popt } & \multicolumn{3}{|c|}{ planar } \\
\hline & $\begin{array}{c}\tilde{\nu} / \\
\mathrm{cm}^{-1}\end{array}$ & $\begin{array}{l}I / \\
\frac{\mathrm{km}}{\mathrm{mol}}\end{array}$ & $\begin{array}{c}D_{\mathrm{e}} / \\
\frac{\mathrm{kJ}}{\mathrm{mol}}\end{array}$ & $\begin{array}{c}\tilde{\nu} / \\
\mathrm{cm}^{-1}\end{array}$ & $\begin{array}{l}I / \\
\frac{\mathrm{km}}{\mathrm{mol}}\end{array}$ & $\begin{array}{c}D_{\mathrm{e}} / \\
\frac{\mathrm{kJ}}{\mathrm{mol}}\end{array}$ \\
\hline \multirow[t]{3}{*}{ cyclisches Trimer } & 3650.3 & 487 & 68.2 & 3711.1 & 366 & 62.1 \\
\hline & 3641.6 & 525 & & 3711.1 & 366 & \\
\hline & 3578.3 & 10 & & 3675.7 & 0 & \\
\hline \multirow[t]{3}{*}{ offenes Trimer } & 3797.6 & 22 & 51.6 & 3824.1 & 12 & 43.2 \\
\hline & 3681.3 & 336 & & 3730.5 & 150 & \\
\hline & 3644.8 & 404 & & 3701.2 & 546 & \\
\hline \multirow[t]{4}{*}{ cyclisches Tetramer } & 3529.8 & 22 & 119.6 & 3625.8 & 0 & 106.7 \\
\hline & 3489.7 & 1397 & & 3603.5 & 1077 & \\
\hline & 3489.6 & 1396 & & 3603.4 & 1077 & \\
\hline & 3393.5 & 0 & & 3547.2 & 0 & \\
\hline \multirow{4}{*}{ offenes Tetramer } & 3813.9 & 15 & 79.4 & 3823.7 & 13 & 69.6 \\
\hline & 3667.9 & 375 & & 3713.0 & 291 & \\
\hline & 3644.2 & 267 & & 3694.2 & 121 & \\
\hline & 3574.6 & 955 & & 3650.8 & 925 & \\
\hline
\end{tabular}




\begin{tabular}{|c|c|c|c|c|}
\hline \multicolumn{5}{|c|}{ Zu: Abbildung 4.10, MP2/aug-cc-pVTZ } \\
\hline$r_{1} / \AA$ & $r_{2} / \AA$ & $\tilde{\nu} / \mathrm{cm}^{-1}$ & $I / \frac{\mathrm{km}}{\mathrm{mol}}$ & $E / E_{\mathrm{h}}$ \\
\hline-0.10 & -0.15 & 3502.2 & 1349 & -305.3580263 \\
\hline-0.10 & -0.10 & 3486.0 & 1419 & -305.3583686 \\
\hline-0.10 & -0.05 & 3475.6 & 1459 & -305.3585119 \\
\hline-0.10 & 0.00 & 3471.4 & 1466 & -305.3584612 \\
\hline-0.10 & 0.05 & 3473.6 & 1439 & -305.3582253 \\
\hline-0.10 & 0.10 & 3482.6 & 1380 & -305.3578161 \\
\hline$-\overline{-0.0 \overline{5}}$ & $-0.2 \overline{5}$ & $3 \overline{5} \overline{5} 1.4$ & $\overline{1} \overline{1} \overline{2} 4$ & $-\overline{3} 0 \overline{5} . \overline{3} \overline{5} 8 \overline{2} 5 \overline{6} \overline{4}$ \\
\hline-0.05 & -0.20 & 3525.0 & 1244 & -305.3591991 \\
\hline-0.05 & -0.15 & 3503.9 & 1342 & -305.3599304 \\
\hline-0.05 & -0.10 & 3487.1 & 1415 & -305.3604344 \\
\hline-0.05 & -0.05 & 3477.0 & 1454 & -305.3607023 \\
\hline-0.05 & 0.00 & 3473.9 & 1458 & -305.3607314 \\
\hline-0.05 & 0.05 & 3477.6 & 1424 & 605260 \\
\hline-0.05 & 0.10 & & 1357 & -30 \\
\hline-0.05 & 0.15 & 3505.5 & 1263 & -305.3594605 \\
\hline-0.05 & 0.20 & 3528.0 & 1151 & 6348 \\
\hline $0.0 \overline{0}$ & $-0.2 \overline{5}$ & $3 \overline{5} \overline{6} 3.2$ & $10 \overline{6} 4$ & $-\overline{3} 0 \overline{5} . \overline{3} \overline{5} 8 \overline{6} 1 \overline{8} \overline{4}$ \\
\hline 0.00 & -0.20 & 3539.0 & 1182 & 596657 \\
\hline 0.00 & -0.15 & 3515.9 & 1286 & -305.3604948 \\
\hline 0.00 & -0.10 & .9 & 1362 & 10883 \\
\hline 0.00 & -0.05 & 3490.2 & 1402 & -305.3614351 \\
\hline 0.00 & 00 & 3488.3 & 1400 & 15299 \\
\hline 0.00 & 0.05 & 3494.4 & 1359 & 3717 \\
\hline 0.00 & 10 & 3506.8 & 1285 & 609660 \\
\hline 0.00 & 0.15 & 3523.4 & 1191 & 3260 \\
\hline 0.00 & 20 & 3546.2 & 1078 & 4713 \\
\hline 0.00 & 0.25 & 3570.3 & 959 & 584243 \\
\hline $0.0 \overline{5}$ & $-0 . \overline{5}$ & $35 \overline{8} 3.3$ & $\overline{9} \overline{6} 6$ & $\overline{5} \overline{9} \overline{8} \overline{2} \overline{8}$ \\
\hline 0.05 & $U$ & & 079 & \\
\hline 0.05 & -( & & 1182 & 98735 \\
\hline 0.05 & -0.10 & & 1264 & 788 \\
\hline 0.05 & -0.05 & 3512.8 & 1308 & 608548 \\
\hline 0.05 & 0.00 & 2.5 & 1300 & .0048 \\
\hline 0.05 & 05 & 3520.5 & 1248 & 609178 \\
\hline 0.05 & 10 & 3534.7 & 1170 & 5818 \\
\hline 0.05 & 15 & 3551.7 & 1077 & 599997 \\
\hline 0.05 & .20 & 3572.6 & 973 & 591867 \\
\hline 0.05 & 0.25 & 3594.1 & 866 & 81657 \\
\hline $0 . \overline{0}$ & $-0.1 \overline{5}$ & $3 \overline{5} \overline{6} 7.9$ & $10 \overline{39}$ & $\overline{5} 8 \overline{2} \overline{7} 6 \overline{9}$ \\
\hline 0.10 & -0.10 & 3552.0 & 1124 & 588116 \\
\hline 0.10 & -0.05 & 3541.9 & 1180 & -305.3591531 \\
\hline 0.10 & 0.00 & 3544.2 & 1163 & -305.3593536 \\
\hline 0.10 & 0.05 & 3553.8 & 1100 & -305.3593732 \\
\hline 0.10 & 0.10 & 3567.6 & 1025 & -305.3591533 \\
\hline 0.10 & 0.15 & 3583.8 & 939 & -305.3586806 \\
\hline 0.10 & 0.20 & 3601.9 & 849 & -305.3579659 \\
\hline
\end{tabular}




\begin{tabular}{|c|c|c|c|c|}
\hline \multicolumn{5}{|c|}{ Zu: Abbildung 4.15 rechts, B3LYP/6-311++G** } \\
\hline Aggregat & Isotopologes & $\tilde{\nu} / \mathrm{cm}^{-1}$ & $I / \frac{\mathrm{km}}{\mathrm{mol}}$ & $f$ \\
\hline \multirow[t]{9}{*}{ cyc. Trimere } & \multirow{3}{*}{$\left(\mathrm{D}_{2} \mathrm{O}\right)_{3}$} & 2630.5 & 257 & 0.9878 \\
\hline & & 2623.1 & 283 & 0.9878 \\
\hline & & 2582.9 & 8 & 0.9878 \\
\hline & \multirow{3}{*}{$\left(\mathrm{D}_{2} \mathrm{O}\right)_{2}\left(\mathrm{H}_{2} \mathrm{O}\right)_{1}$} & 3600.6 & 387 & 0.9762 \\
\hline & & 2628.4 & 258 & 0.9878 \\
\hline & & 2597.8 & 94 & 0.9878 \\
\hline & \multirow[t]{3}{*}{$\left(\mathrm{D}_{2} \mathrm{O}\right)_{1}\left(\mathrm{H}_{2} \mathrm{O}\right)_{2}$} & 3629.4 & 517 & 0.9762 \\
\hline & & 3586.5 & 215 & 0.9762 \\
\hline & & 2606.0 & 184 & 0.9878 \\
\hline \multirow[t]{12}{*}{ offene- Trimere- } & \multirow{3}{*}{$\overline{\mathrm{D}_{2}} \overline{\mathrm{O}}-\overline{\mathrm{D}_{2}} \overline{\mathrm{O}}-\overline{\mathrm{D}_{2}} \overline{\mathrm{O}}$} & $\overline{2} \overline{6} \overline{25.4}$ & $\overline{2} \overline{3} 0^{-}$ & $0 . \overline{9} 8 \overline{7} \overline{8}$ \\
\hline & & 2650.4 & 183 & 0.9878 \\
\hline & & 2742.1 & 16 & 0.9878 \\
\hline & \multirow{3}{*}{$\mathrm{D}_{2} \mathrm{O}-\mathrm{D}_{2} \mathrm{O}-\mathrm{H}_{2} \mathrm{O}$} & 2625.6 & 232 & 0.9878 \\
\hline & & 2650.6 & 181 & 0.9878 \\
\hline & & 3802.7 & 28 & 0.9762 \\
\hline & \multirow[t]{3}{*}{$\mathrm{D}_{2} \mathrm{O}-\mathrm{H}_{2} \mathrm{O}-\mathrm{D}_{2} \mathrm{O}$} & 2634.5 & 211 & 0.9878 \\
\hline & & 2741.7 & 19 & 0.9878 \\
\hline & & 3647.1 & 404 & 0.9762 \\
\hline & \multirow[t]{3}{*}{$\mathrm{H}_{2} \mathrm{O}-\mathrm{D}_{2} \mathrm{O}-\mathrm{D}_{2} \mathrm{O}$} & 2641.0 & 203 & 0.9878 \\
\hline & & 2742.1 & 15 & 0.9878 \\
\hline & & 3636.2 & 427 & 0.9762 \\
\hline \multirow[t]{6}{*}{$\overline{\text { Lasso }}$} & \multirow[t]{6}{*}{$\overline{\left(\mathrm{D}_{2} \mathrm{O}\right)_{4}} \cdots \overline{\mathrm{OH}_{2}}$} & $2 \overline{4} \overline{2} 5.3$ & $\overline{13} \overline{2}^{-}$ & $0 . \overline{9} 9 \overline{2} \overline{6}$ \\
\hline & & 2473.8 & 910 & 0.9926 \\
\hline & & 2504.3 & 280 & 0.9926 \\
\hline & & 2667.1 & 127 & 0.9926 \\
\hline & & 2746.7 & 100 & 0.9926 \\
\hline & & 3629.1 & 367 & 0.9804 \\
\hline
\end{tabular}

\begin{tabular}{|c|c|c|c|c|}
\hline \multicolumn{5}{|c|}{$\begin{array}{l}\text { Zu: Abbildungen } 5.2 \text { und } 5.3 \text { oben, } \\
\text { MP2/6-311+G* }\end{array}$} \\
\hline Aggregat & $\tilde{\nu} / \mathrm{cm}^{-1}$ & $I / \frac{\mathrm{km}}{\mathrm{mol}}$ & $\overline{E_{0} / E_{\mathrm{h}}}$ & $D_{0} / \frac{\mathrm{kJ}}{\mathrm{mol}}$ \\
\hline Monomer & 3678.0 & 66 & $\overline{-225.538199}$ & \\
\hline \multirow[t]{2}{*}{ Dimer } & 3387.0 & 1227 & -451.092415 & 42.1 \\
\hline & 3674.0 & 82 & & \\
\hline \multirow[t]{3}{*}{ cyc. Trimer } & 3412.9 & 506 & -676.658309 & 114.8 \\
\hline & 3505.1 & 369 & & \\
\hline & 3558.8 & 238 & & \\
\hline \multirow[t]{3}{*}{ offenes Trimer } & 3311.3 & 266 & -676.649866 & 92.6 \\
\hline & 3327.5 & 326 & & \\
\hline & 3672.4 & 87 & & \\
\hline
\end{tabular}




\begin{tabular}{|c|c|c|c|c|}
\hline \multicolumn{5}{|c|}{$\begin{array}{l}\text { Zu: Abbildungen } 5.3 \text { unten und } 5.4 \\
\text { MP2/aug-cc-pVDZ }\end{array}$} \\
\hline Aggregat & $\tilde{\nu} / \mathrm{cm}^{-1}$ & $I / \frac{\mathrm{km}}{\mathrm{mol}}$ & $E_{0} / E_{\mathrm{h}}$ & $D_{0} / \frac{\mathrm{kJ}}{\mathrm{mol}}$ \\
\hline $\bar{i}$ & 3662.4 & 71 & -225.528961 & \\
\hline \multirow[t]{2}{*}{$i i$} & 3319.5 & 7 & -451.074810 & 44.3 \\
\hline & 3657.9 & 89 & & \\
\hline \multirow[t]{2}{*}{$w$} & 3803.3 & 4 & -76.239579 & \\
\hline & 3937.6 & 67 & & \\
\hline \multirow[t]{3}{*}{$w i$} & 3574.3 & 650 & -301.778638 & 26.5 \\
\hline & 3661.7 & 78 & & \\
\hline & 3891.5 & 96 & & \\
\hline \multirow[t]{3}{*}{$i w$} & 3537.0 & 582 & -301.777531 & 23.6 \\
\hline & 3798.2 & 16 & & \\
\hline & 3928.6 & 95 & & \\
\hline \multirow[t]{3}{*}{$i^{18} w$} & 3537.0 & 582 & -301.777624 & 23.7 \\
\hline & 3790.1 & 15 & & \\
\hline & 3912.6 & 94 & & \\
\hline \multirow[t]{3}{*}{${ }^{18} w i$} & 3564.1 & 650 & -301.778726 & 26.6 \\
\hline & 3661.7 & 78 & & \\
\hline & 3878.1 & 89 & & \\
\hline$m$ & 3840.4 & 34 & -115.370344 & \\
\hline \multirow[t]{2}{*}{$\mathrm{mm}$} & 3675.3 & 483 & -230.748854 & 21.4 \\
\hline & 3830.1 & 45 & & \\
\hline \multirow[t]{2}{*}{$i m$} & 3493.4 & 658 & -340.910814 & 30.2 \\
\hline & 3839.6 & 52 & & \\
\hline \multirow[t]{2}{*}{$m i$} & 3579.4 & 742 & -340.911420 & 31.8 \\
\hline & 3660.7 & 78 & & \\
\hline
\end{tabular}

Zu: Abbildungen 5.5 und 5.6 MP2/aug-cc-pVDZ

\begin{tabular}{lcrcc}
\hline Aggregat & $\tilde{\nu} / \mathrm{cm}^{-1}$ & $I / \frac{\mathrm{km}}{\mathrm{mol}}$ & $E_{0} / E_{\mathrm{h}}$ & $D_{0} / \frac{\mathrm{kJ}}{\mathrm{mol}}$ \\
\hline \hline $4 p$ & 3658.0 & 94 & -455.830566 & \\
$5 p$ & 3644.1 & 54 & -455.829110 & \\
$w$ & 3803.3 & 4 & -76.239579 & \\
$4 p w$ & 3937.6 & 67 & & \\
& 3522.9 & 776 & -532.079767 & 25.3 \\
& 3798.9 & 19 & & \\
$5 p w$ & 3929.5 & 96 & & \\
& 3501.2 & 336 & -532.080392 & 30.7 \\
& 3740.1 & 61 & & \\
$w 4 p$ & 3893.3 & 135 & & \\
& 3528.6 & 705 & -532.081087 & 28.7 \\
& 3653.4 & 100 & & \\
$w 5 p$ & 3882.6 & 87 & & \\
& 3571.8 & 842 & -532.078927 & 26.9 \\
& 3642.6 & 58 & & \\
\hline
\end{tabular}




\section{A.5 Experimentelle Parameter}

\section{A.5.1 Muesli-Jet}

\section{Allgemeine Parameter}

\begin{tabular}{llll}
\hline Instrument-Parameter & & & \\
\hline Spectrometer & Vertex 70 v & & \\
\hline Optik-Parameter & & & 0 \\
\hline Beamsplitter & KBr & Preamplifier Gain & MIR \\
Measurement Channel & Right Exit & Source Setting & \\
High Pass Filter & Open & & \\
\hline Akquisitions-Parameter & Single Sided & Delay Before Measurement & 0 \\
\hline Acquisition Mode & 0 & Stabilization Delay & 0 \\
Correlation Test Mode & 2 & & \\
Signal Gain & & & No \\
\hline FT-Parameter & 0 & Stored Phase Mode & 4 \\
\hline Non Linearity Correction & 0 & Zero Filling Factor & \\
Phase Correction Mode & Mertz & & \\
\hline
\end{tabular}

Parameterblöcke für trägergashaltige Expansionen

\begin{tabular}{llll}
\hline $\begin{array}{l}\text { Parameterblock } \\
\text { muesliJET-*.xpm }\end{array}$ & $\begin{array}{l}\text { M-I } \\
\text { InSb }\end{array}$ & $\begin{array}{l}\text { M-M } \\
\text { MCT }\end{array}$ & $\begin{array}{l}\text { M-MI } \\
\text { MCT-InSb }\end{array}$ \\
\hline Optik-Parameter & & & \\
\hline Apertur & $6 \mathrm{~mm}$ & $6 \mathrm{~mm}$ & $6 \mathrm{~mm}$ \\
Detector Setting & LN-InSb & LN-MCT Mid & LN-MCT Mid + LN-InSb \\
Low Pass Filter & 20 & 20 & 20 \\
Scanner Velocity & $80 \mathrm{kHz}$ & $80 \mathrm{kHz}$ & $80 \mathrm{kHz}$ \\
\hline Akquisitions-Parameter & & & \\
\hline Wanted High Frequency Limit & 5000 & 3000 & 5000 \\
Wanted Low Frequency Limit & 0 & 0 & 0 \\
Resolution & 2 & 2 & 2 \\
Sample Scans & 1 & 1 & 1 \\
Background Scans & 20 & 20 & 20 \\
\hline FT-Parameter & & & \\
\hline Apodization Function & BH3 & BH3 & BH3 \\
Phase Resolution & 32 & 32 & 32 \\
\hline
\end{tabular}


Parameterblöcke für trägergasfreie Expansionen

\begin{tabular}{llll}
\hline $\begin{array}{l}\text { Parameterblock } \\
\text { muesliJET-*.xpm }\end{array}$ & M-W1/20 & M-W1/10 & M-W05/20 \\
Optik-Parameter & & & \\
\hline Apertur & $4 \mathrm{~mm}$ & $4 \mathrm{~mm}$ & $3 \mathrm{~mm}$ \\
Detector Setting & LN-InSb & LN-InSb & LN-InSb \\
Low Pass Filter & 40 & 40 & 40 \\
Scanner Velocity & $120 \mathrm{kHz}$ & $120 \mathrm{kHz}$ & $120 \mathrm{kHz}$ \\
\hline Akquisitions-Parameter & & & \\
\hline Wanted High Frequency Limit & 4500 & 4500 & 4500 \\
Wanted Low Frequency Limit & 2700 & 2700 & 2700 \\
Resolution & 1 & 1 & 0.5 \\
Sample Scans & 1 & 1 & 1 \\
Background Scans & 20 & 10 & 20 \\
\hline FT-Parameter & & & \\
\hline Apodization Function & $\mathrm{NBM}$ & $\mathrm{NBM}$ & $\mathrm{NBM}$ \\
Phase Resolution & 4 & 4 & 4 \\
\hline
\end{tabular}

\section{A.5.2 Popcorn-Jet}

\section{Experiment INSB4DSFR.xpm (INSB35DSFR.xpm)}

\begin{tabular}{|c|c|c|c|}
\hline \multicolumn{4}{|l|}{ Instrument-Parameter } \\
\hline Spectrometer & IFS $66 \mathrm{v} / \mathrm{S}$ & Detector & InSb $2 \mathrm{~mm} \mathrm{neu}$ \\
\hline \multicolumn{4}{|l|}{ Optik-Parameter } \\
\hline Apertur & $4 \mathrm{~mm}(3.5 \mathrm{~mm})$ & Low Pass Filter & Open \\
\hline Beamsplitter & $\mathrm{KBr}$ & Source Setting & MIR Source \\
\hline Measurement Channel & Hüllstrom/RESS & Scanner Velocity & $80 \mathrm{kHz}$ \\
\hline Detector Setting & External A & & \\
\hline \multicolumn{4}{|c|}{ Akquisitions-Parameter } \\
\hline Acquisition Mode & Double Sided & High Freq. Limit & 15799.83 \\
\hline Correlation Test Mode & No & Low Freq. Limit & 0 \\
\hline Delay Before Measurement & 0 & Signal Gain & Automatic \\
\hline Stabilization Delay & 2 & Sample Scans & 1 \\
\hline Resolution & 2 & Background Scans & 20 \\
\hline \multicolumn{4}{|l|}{ FT-Parameter } \\
\hline Apodization Function & NMB & Phase Correction Mode & Mertz \\
\hline Non Linearity Correction & 0 & Stored Phase Mode & No \\
\hline Phase Resolution & 16 & Zero Filling Factor & 4 \\
\hline
\end{tabular}




\section{A.5.3 Chilli-Jet}

\section{Allgemeine Parameter}

\begin{tabular}{llll}
\hline Instrument-Parameter & & & \\
\hline Spectrometer & Vertex 70 v & Detector & MCT-FPA \\
\hline Optik-Parameter & & & \\
\hline Beamsplitter & KBr & Scanner Velocity & 5 \\
Measurement Channel & Front Exit & Imaging Device & Macro Chamber \\
$\begin{array}{l}\text { Detector Setting } \\
\text { Source Setting }\end{array}$ & Int. Pos. 1 & Objective & Macro Chamber \\
\hline Akquisitions-Parameter & & & \\
\hline Delay Before Measurement & 0 & Signal Gain & 1 \\
Stabilization Delay & 0 & High Freq. Limit & 3900 \\
Binning & No binning & Low Freq. Limit & 0 \\
\hline FT-Parameter & & & \\
\hline Phase Correction Mode & Mertz & Zero Filling Factor & 2 \\
\hline
\end{tabular}

\section{Variable Parameter}

\begin{tabular}{lllll}
\hline $\begin{array}{l}\text { Parameterblock } \\
\text { *.xpm }\end{array}$ & $\begin{array}{l}\text { C-P4 } \\
\text { FPAPuls }\end{array}$ & $\begin{array}{l}\text { C-P2 } \\
\sim 2 \mathrm{cmSsFb}\end{array}$ & $\begin{array}{l}\text { C-K4 } \\
\text { machdisk }\end{array}$ & $\begin{array}{l}\text { C-K2 } \\
\text { Signaltest-FPA-2cm }\end{array}$ \\
\hline Optik-Parameter & & & & \\
\hline Apertur & $8 \mathrm{~mm}$ & $6 \mathrm{~mm}$ & $8 \mathrm{~mm}$ & $6 \mathrm{~mm}$ \\
\hline \multicolumn{2}{l}{ Akquisitions-Parameter } & & & \\
\hline Acquisition Mode & $\mathrm{DsFb}$ & $\mathrm{SsFb}$ & $\mathrm{DsFb}$ & $\mathrm{SsFb}$ \\
Resolution & 4 & 2 & 4 & 2 \\
Sample Scans & 1 & 1 & - & - \\
Background Scans & 20 & 20 & - & - \\
\hline FT-Parameter & & & & \\
\hline Apodization Function & $\mathrm{NBM}$ & $\mathrm{BH} 3$ & $\mathrm{BH} 3$ & $\mathrm{BH} 3$ \\
Phase Resolution & 16 & 32 & 16 & 16 \\
\hline
\end{tabular}

\section{A.6 Pulssynchronisation}

\section{A.6.1 TRS-Methode}

In der TRS-Methode wird die Messabfolge für eine zeitaufgelöste Aufnahme von Scansequenzen festgelegt. Der Syntax ist dabei wie folgt festgelegt:

\begin{tabular}{ll}
\hline Befehl & Bedeutung \\
\hline \hline Set output high/low $n$ & TTL-Signal am Eingang $n$ auf $1 / 0$ setzen \\
Comment „text" & Kommentar \\
Set buffer $n$ & Messung an Position $n$ der Multispektrendatei speichern \\
Measure $n$ & $n$ Spektren messen \\
\hline
\end{tabular}




\section{A.7 Makros}

\section{A.7.1 Muesli-Jet}

\section{Messmakro muesliMEAS.mtx}

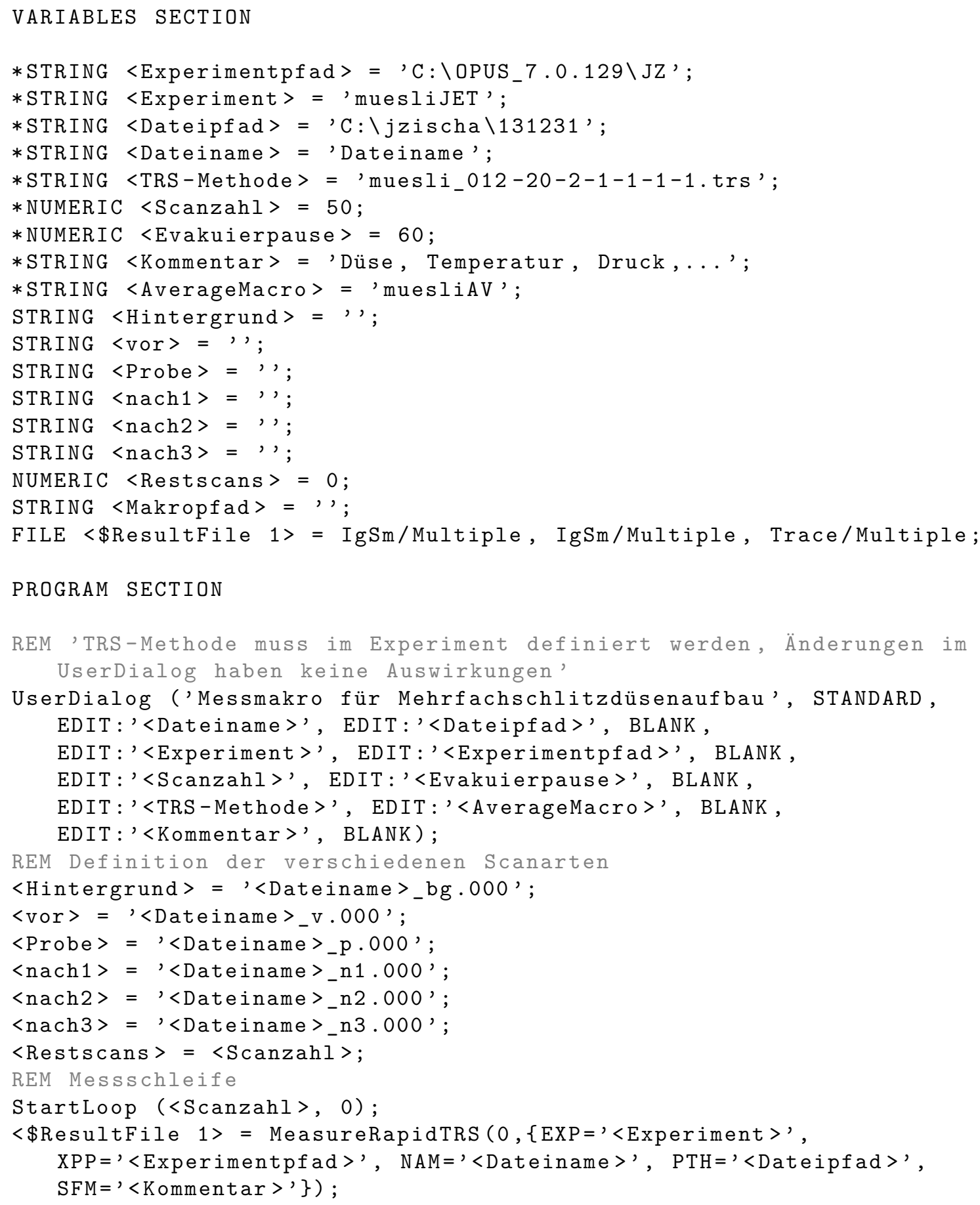


PostrunExtract ( $[<\$ R e s u l t F i l e 1>:$ IgSm/Multiple $], \quad\{E X S=0, \quad E X E=0$, $\mathrm{ENT}=0, \mathrm{ENE}=0, \mathrm{ECO}=0, \mathrm{XTP}=$ '<Dateipfad $\rangle^{\prime}, \mathrm{XTN}=$ ' $\langle\text { Hintergrund }\rangle^{\prime}$, $\left.\left.\mathrm{XTI}=0, \mathrm{EAB}=0, \mathrm{ELF}=0, \mathrm{EIL}=0^{\prime}, \mathrm{EUL}=1, \mathrm{EDM}=1\right\}\right)$;

PostrunExtract ( $[<\$ R$ ResultFile 1 $>$ : IgSm/Multiple $], \quad\{E X S=0, E X E=0$, $\mathrm{ENT}=1, \mathrm{ENE}=1, \mathrm{ECO}=0, \mathrm{XTP}=$ ' $^{2}$ Dateipfad $>$ ', XTN='<vor $>$ ', XTI=0, $\left.\left.\mathrm{EAB}=0, \mathrm{ELF}=0, \mathrm{EIL}={ }^{\prime}{ }^{\prime}, \mathrm{EUL}=1, \mathrm{EDM}=1\right\}\right)$;

PostrunExtract ( $[<\$ R e s u l t F i l e$ 1 $>$ : IgSm/Multiple $], \quad\{E X S=0, E X E=0$, $\mathrm{ENT}=2, \mathrm{ENE}=2, \mathrm{ECO}=0, \mathrm{XTP}={ }^{\prime}\langle\text { Dateipfad }\rangle^{\prime}, \mathrm{XTN}={ }^{\prime}\langle$ Probe $\rangle$ ', $\mathrm{XTI}=0$, $\left.\left.\mathrm{EAB}=0, \quad \mathrm{ELF}=0, \mathrm{EIL}={ }^{\prime}{ }^{\prime}, \mathrm{EUL}=1, \mathrm{EDM}=1\right\}\right)$;

PostrunExtract ( $[<\$ R e s u l t F i l e ~ 1>:$ IgSm/Multiple $], \quad\{E X S=0, E X E=0$, $\mathrm{ENT}=3, \mathrm{ENE}=3, \mathrm{ECO}=0, \mathrm{XTP}={ }^{\prime}<$ Dateipfad $\left.\rangle^{\prime}, \mathrm{XTN}^{\prime}{ }^{\prime}<\operatorname{nach} 1\right\rangle^{\prime}, \mathrm{XTI}=0$, $\left.\left.\mathrm{EAB}=0, \mathrm{ELF}=0, \mathrm{EIL}={ }^{\prime}{ }^{\prime}, \mathrm{EUL}=1, \mathrm{EDM}=1\right\}\right)$;

PostrunExtract ( $[<\$ R$ ResultFile $1>$ : IgSm/Multiple $],\{E X S=0, E X E=0$, $\mathrm{ENT}=4, \mathrm{ENE}=4, \mathrm{ECO}=0, \mathrm{XTP}={ }^{\prime}<$ Dateipfad $\left.\rangle^{\prime}, \mathrm{XTN}={ }^{\prime}<\mathrm{nach} 2\right\rangle^{\prime}, \mathrm{XTI}=0$, $\left.\left.\mathrm{EAB}=0, \mathrm{ELF}=0, \mathrm{EIL}={ }^{\prime} \mathbf{\prime}^{\prime}, \mathrm{EUL}=1, \mathrm{EDM}=1\right\}\right)$;

PostrunExtract ( $[<\$ R e s u l t F i l e ~ 1>:$ IgSm/Multiple $], \quad\{E X S=0, E X E=0$, $\mathrm{ENT}=5, \mathrm{ENE}=5, \mathrm{ECO}=0, \mathrm{XTP}={ }^{\prime}\langle\text { Dateipfad }\rangle^{\prime}, \mathrm{XTN}={ }^{\prime}\langle\operatorname{nach} 3\rangle^{\prime}, \mathrm{XTI}=0$, $\left.\left.\mathrm{EAB}=0, \mathrm{ELF}=0, \mathrm{EIL}=5^{\prime}, \mathrm{EUL}=1, \mathrm{EDM}=1\right\}\right)$;

Unload ( $[<$ ResultFile 1>:IgSm/Multiple $],\{\})$;

$\langle$ Restscans $>=\langle$ Restscans $>-1$;

Message ('Noch <Restscans> von <Scanzahl> Scans', ON_SCREEN, $<$ Evakuierpause >);

EndLoop (0);

REM Aufrufen und Durchführen des Spektrenmittelungsmakros

$<$ Makropfad $>$ GetMacroPath ();

CallMacro ('<Makropfad $>\backslash<$ AverageMacro>.mtx', $\{$ '<Dateipfad ' , '<Dateiname $>$ ' $\},\{\})$;

Message ('Messung beendet', ON_SCREEN, NO_TIMEOUT);

\section{PARAMETER SECTION}

INP $=$ C $: \backslash O P U S \_7.0 .129 \backslash$ METHODS ;

IFP $=$ C : \OPUS $\_7.0 .129 \backslash$ METHODS ;

INM $=$ DEFAULT;

IFN $=$ DEFAULT;

$\mathrm{DPA}=5$;

$\mathrm{DPO}=8$;

$\mathrm{SEP}=$, ; 


\section{Spektrenmittelungsmakro muesliAV.mtx}

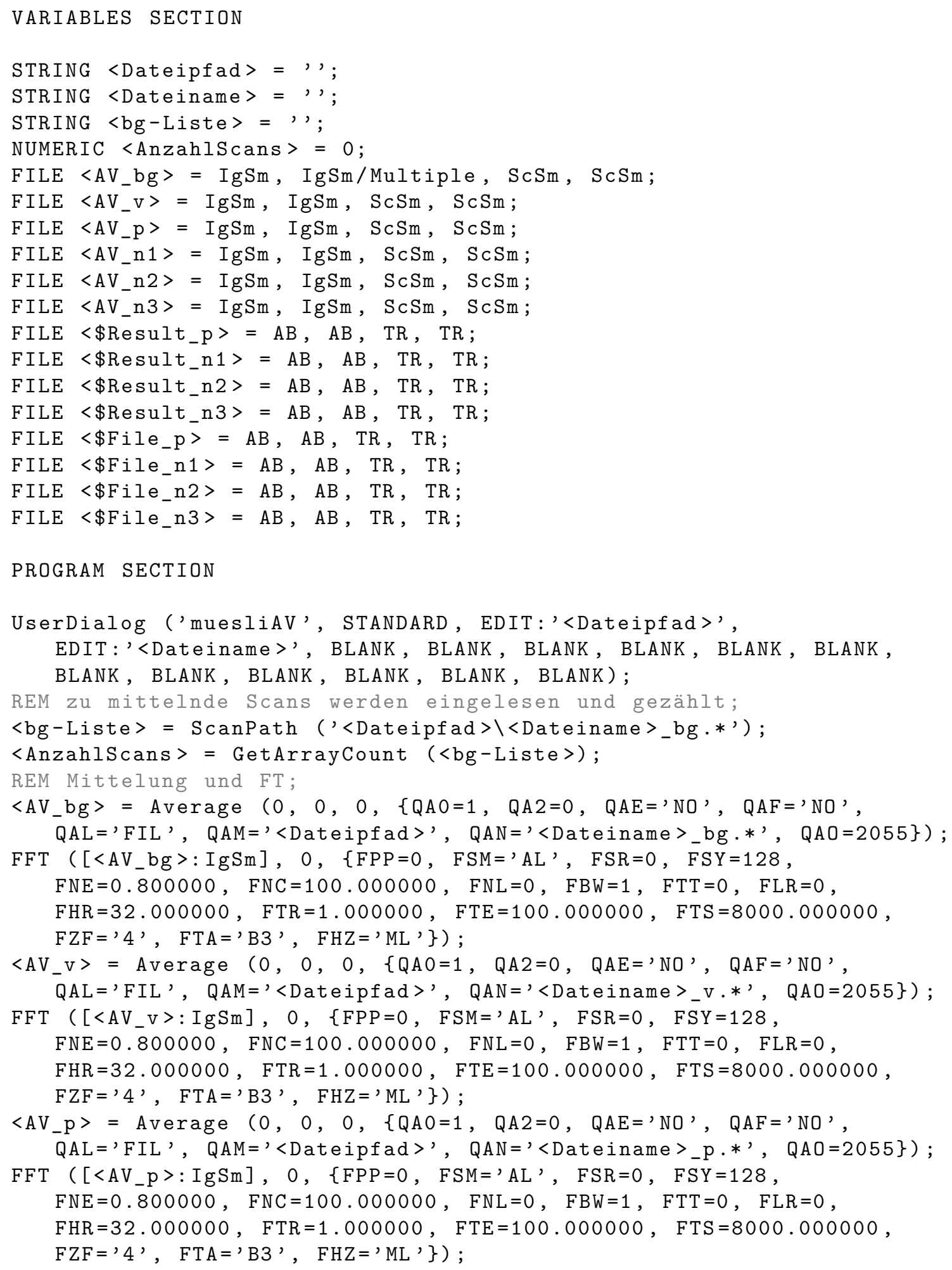


$\left\langle A V_{-} n 1>=\right.$ Average $\left(0,0,0,\left\{Q A 0=1, Q A 2=0, Q A E={ }^{\prime} N O{ }^{\prime}, Q A F=\right.\right.$ 'NO', $\mathrm{QAL}=$ 'FIL', $\mathrm{QAM}=$ '<Dateipfad >', $\mathrm{QAN}=$ '<Dateiname>_n1.*', QAO=2055\});

FFT ( $\left[<A V_{-} n 1>: I g S m\right], 0,\left\{F P P=0, F S M={ }^{\prime} A L^{\prime}, F S R=0, \quad F S Y=128\right.$, $\mathrm{FNE}=0.800000, \quad F N C=100.000000, F N L=0, F B W=1, F T T=0, F L R=0$, $\mathrm{FHR}=32.000000, \mathrm{FTR}=1.000000, \mathrm{FTE}=100.000000, \mathrm{FTS}=8000.000000$, $\left.\left.\mathrm{FZF}={ }^{\prime} 4, \quad \mathrm{FTA}={ }^{\prime} \mathrm{B} 3{ }^{\prime}, \mathrm{FHZ}={ }^{\prime} \mathrm{ML}{ }^{\prime}\right\}\right)$;

$\left\langle A V_{-} n 2>=\right.$ Average $\left(0,0,0,\left\{Q A 0=1, Q A 2=0, Q A E={ }^{\prime} N O{ }^{\prime}, Q A F={ }^{\prime} N O^{\prime}\right.\right.$, $\mathrm{QAL}=$ 'FIL', $\mathrm{QAM}=$ '<Dateipfad>', $\mathrm{QAN}=$ '<Dateiname>_n2.*', $\mathrm{QAO}=2055\})$;

FFT ( $\left[<A V_{-} n 2>: \operatorname{IgSm}\right], 0,\{F P P=0, F S M=' A L ', F S R=0, F S Y=128$, $\mathrm{FNE}=0.800000, \quad \mathrm{FNC}=100.000000, \mathrm{FNL}=0, \mathrm{FBW}=1, \mathrm{FTT}=0, \mathrm{FLR}=0$, $\mathrm{FHR}=32.000000, \mathrm{FTR}=1.000000, \mathrm{FTE}=100.000000, \mathrm{FTS}=8000.000000$, $\mathrm{FZF}={ }^{\prime} 4$ ', $\left.\left.\mathrm{FTA}={ }^{\prime} \mathrm{B} 3{ }^{\prime}, \mathrm{FHZ}={ }^{\prime} \mathrm{ML}{ }^{\prime}\right\}\right)$;

$\left\langle A V_{-} n 3>=\right.$ Average $\left(0,0,0,\left\{Q A 0=1, Q A 2=0, Q A E={ }^{\prime} N O^{\prime}, Q A F={ }^{\prime} N O^{\prime}\right.\right.$, QAL='FIL', $Q A M=$ '<Dateipfad >', $Q A N=$ '<Dateiname>_n3.*', $Q A 0=2055\})$;

FFT $\left(\left[<\mathrm{AV} \_n 3>: \operatorname{IgSm}\right], 0,\left\{\mathrm{FPP}=0, \mathrm{FSM}={ }^{\prime} \mathrm{AL},, \mathrm{FSR}=0, \quad \mathrm{FSY}=128\right.\right.$, $\mathrm{FNE}=0.800000, \mathrm{FNC}=100.000000, \mathrm{FNL}=0, \mathrm{FBW}=1, \mathrm{FTT}=0, \mathrm{FLR}=0$, $\mathrm{FHR}=32.000000, \mathrm{FTR}=1.000000, \mathrm{FTE}=100.000000, \mathrm{FTS}=8000.000000$, $\left.\left.\mathrm{FZF}={ }^{\prime} 4{ }^{\prime}, \mathrm{FTA}={ }^{\prime} \mathrm{B} 3^{\prime}, \mathrm{FHZ}={ }^{\prime} \mathrm{ML}{ }^{\prime}\right\}\right)$;

REM Berechnung von Absorptionsipektren;

$\left[\langle\right.$ Result_p $>: T R]=$ Calculator ( $\left[\left\langle\mathrm{AV}_{-} \mathrm{p}\right\rangle: \mathrm{ScSm}\right]\left[\left\langle\mathrm{AV} \mathrm{S}_{-} \mathrm{bg}\right\rangle: \mathrm{ScSm}\right]$, $\left\{\mathrm{FOR}={ }^{\prime}\left[\left\langle\mathrm{AV} \_\mathrm{p}\right\rangle: \mathrm{ScSm}\right] /\left[\left\langle\mathrm{AV}_{-} \mathrm{bg}\right\rangle: \mathrm{ScSm}^{\prime}\right\}\right) ;$

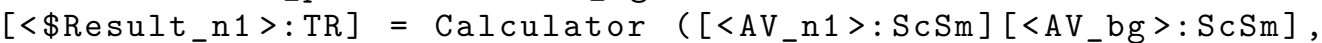
$\left.\left\{\mathrm{FOR}={ }^{\prime}\left[\left\langle\mathrm{AV} \_\mathrm{n} 1\right\rangle: \mathrm{ScSm}\right] /\left[\left\langle\mathrm{AV} \_\mathrm{bg}\right\rangle: \mathrm{ScSm}\right]^{\prime}\right\}\right) ;$

$[<$ Result_n2 $>: \mathrm{TR}]=\mathrm{Calculator}\left(\left[\left\langle\mathrm{AV} \_\mathrm{n} 2\right\rangle: \mathrm{ScSm}\right]\left[\left\langle\mathrm{AV} \_\mathrm{bg}\right\rangle: \mathrm{ScSm}\right]\right.$, $\left.\left\{\mathrm{FOR}={ }^{\prime}\left[\left\langle\mathrm{AV} \_\mathrm{n} 2\right\rangle: \mathrm{ScSm}\right] /\left[\left\langle\mathrm{AV} \_\mathrm{bg}\right\rangle: \mathrm{ScSm}\right]^{\prime}\right\}\right) ;$

$[<$ Result_n3 $>: T R]=$ Calculator $\left(\left[<\mathrm{AV}_{-} \mathrm{n} 3>: \mathrm{ScSm}\right]\left[\left\langle\mathrm{AV} \_\mathrm{bg}\right\rangle: \mathrm{ScSm}\right]\right.$, $\left\{\mathrm{FOR}={ }^{\prime}\left[\langle\mathrm{AV} \mathrm{n} 3>: \mathrm{ScSm}] /\left[\langle\mathrm{AV} \mathrm{bg}>: \mathrm{ScSm}]^{\prime}\right\}\right) ;\right.$

ABTR ( $[<\$ R$ esult_p $>: \mathrm{TR}],\{\mathrm{CCM}=3\})$;

ABTR ( $[<\$ R$ esult_n1 $>: T R],\{\mathrm{CCM}=3\})$;

ABTR ( $\left.\left[<\$ R e s u l t \_n 2>: T R\right],\{\mathrm{CCM}=3\}\right)$;

ABTR ( $\left.\left[<\$ R e s u l t \_n 3>: T R\right], \quad\{\mathrm{CCM}=3\}\right)$;

SaveAs ( $\left[\langle \$\right.$ Result_p $>],\{\text { DAP='<Dateipfad }\rangle^{\prime}, \quad$ OEX $=$ '0',

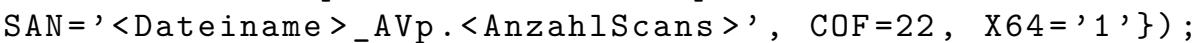

SaveAs ( $\left.\left[<\$ R e s u l t \_n 2\right\rangle\right], \quad\left\{\mathrm{DAP}={ }^{\prime}<\text { Dateipfad }\right\rangle^{\prime}, \quad \mathrm{OEX}={ }^{\prime} 0$ ', $\mathrm{SAN}={ }^{\prime}\left\langle\right.$ Dateiname $>$ AVn2. $\left.\left.\langle\text { AnzahlScans }\rangle^{\prime}, \quad \mathrm{COF}=22, \mathrm{X} 64={ }^{\prime} 1^{\prime}\right\}\right)$;

SaveAs ( $[<\$ R$ Result_n1 $>],\left\{\right.$ DAP='<Dateipfad $>$ ', $0 E X={ }^{\prime} 0$ ',

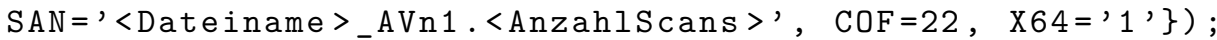
SaveAs ( $\left[\langle \$\right.$ Result_n3 $>],\left\{\mathrm{DAP}={ }^{\prime}<\right.$ Dateipfad $>$ ', $\mathrm{OEX}={ }^{\prime} 0^{\prime}$, , $\mathrm{SAN}=$ '<Dateiname >_AVn3.<AnzahlScans >', COF=22, X64='1'\}); Unload ( $\left.\left[\left\langle\mathrm{AV}_{-} \mathrm{bg}\right\rangle\right],\{\}\right)$;

Unload ( $\left.\left[\left\langle A V_{-} V\right\rangle\right],\{\}\right)$;

Unload ( $\left.\left.\left[<\mathrm{AV}_{-} \mathrm{p}\right\rangle\right],\{\}\right)$;

Unload ( $\left.\left[<\mathrm{AV} \_n 1>\right],\{\}\right)$;

Unload ( $\left.\left.\left[<\mathrm{AV}_{-} \mathrm{n} 2\right\rangle\right],\{\}\right)$;

Unload ( $\left.\left[<A V_{-} n 3>\right],\{\}\right)$;

Unload ( $[\langle \$$ Result_p $\rangle],\{\})$;

Unload ( $[<\$ R$ Result_n1 $\rangle],\{\})$;

Unload ( $[<\$ R$ Result_n2 $\rangle],\{\})$;

Unload ( $[<\$ R$ Result_n3>], \{\});

REM Probenspektrum wird geladen; 


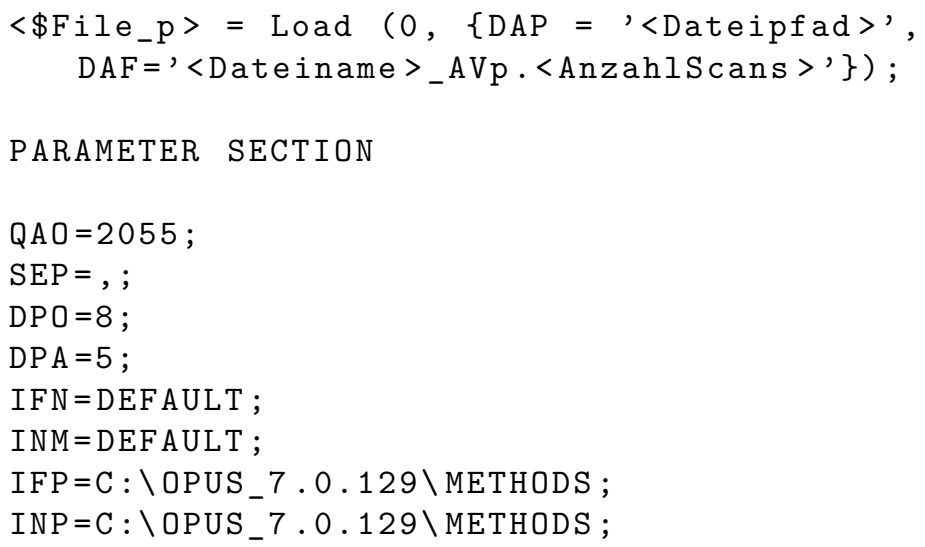

\section{A.7.2 Chilli-Jet}

\section{Messmakro FPACYCLEMEASvar.mtx}

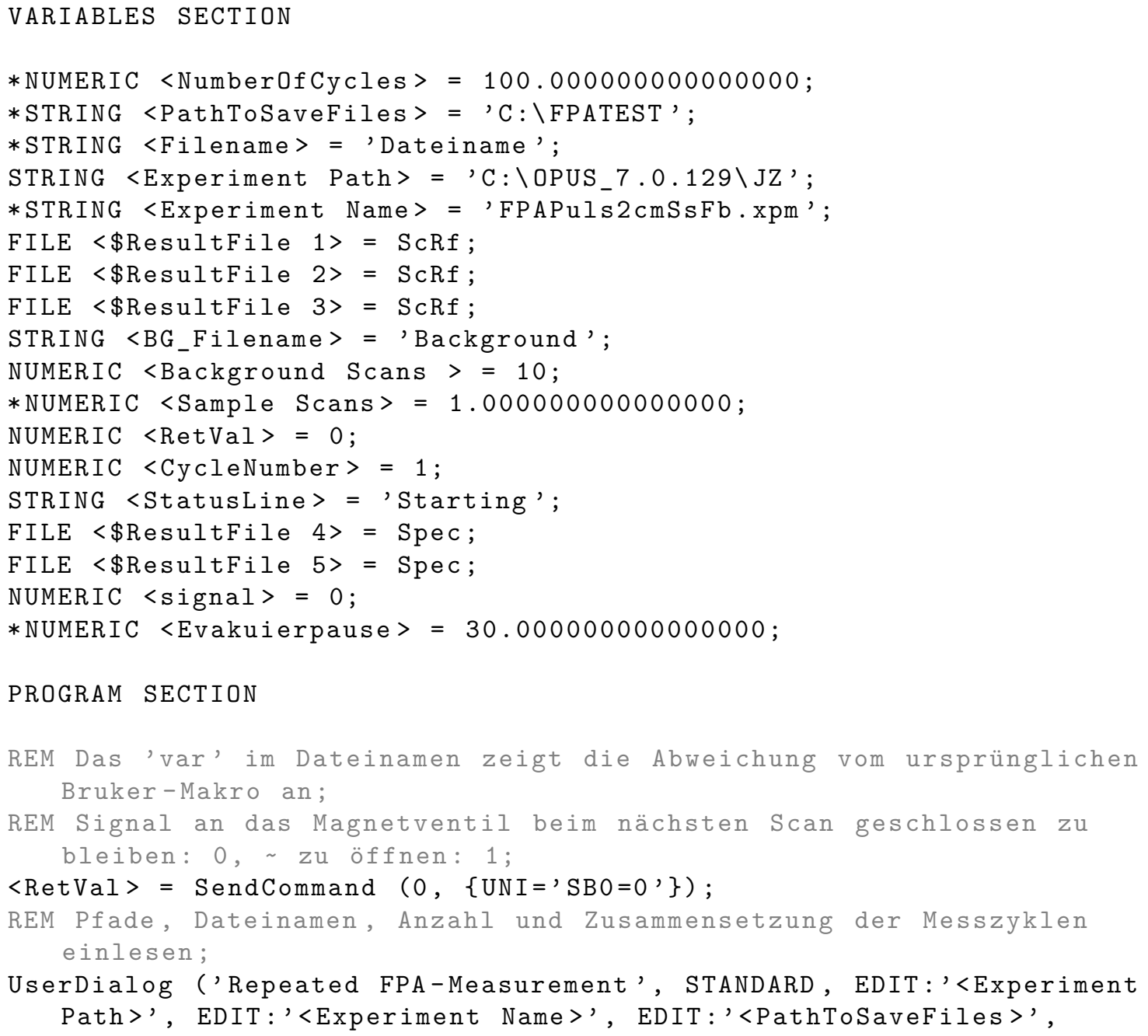




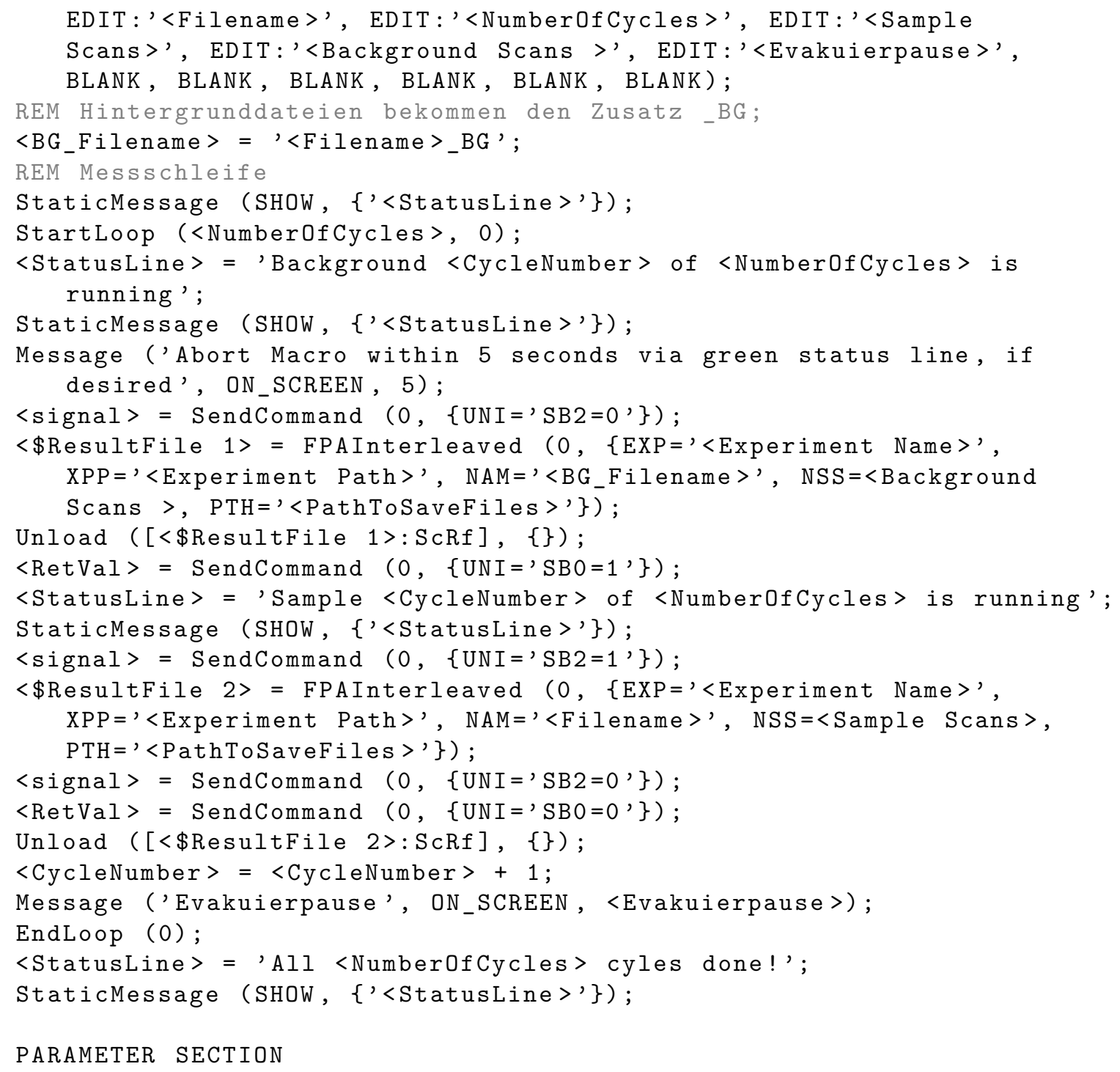

Fouriertransformations- und Mittelungsmakro chilliFT.mtx

VARIABLES SECTION

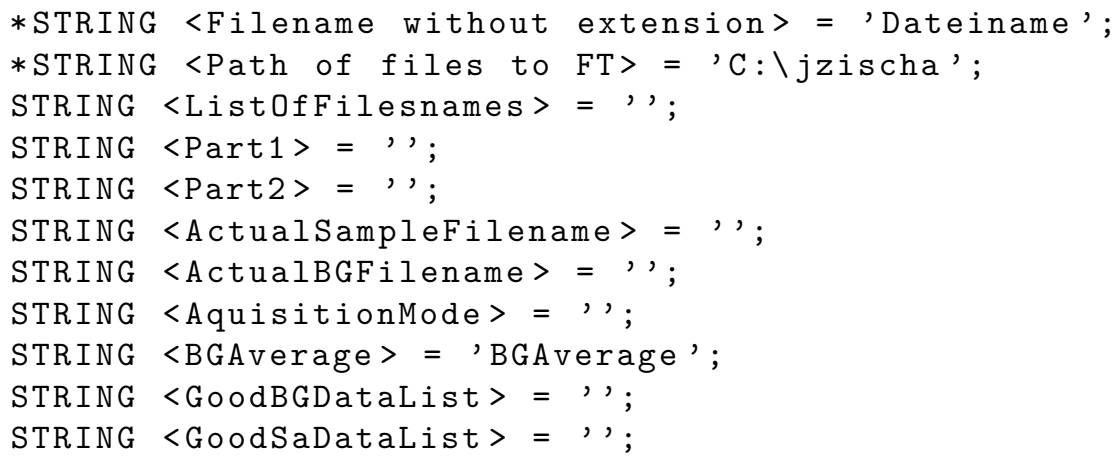




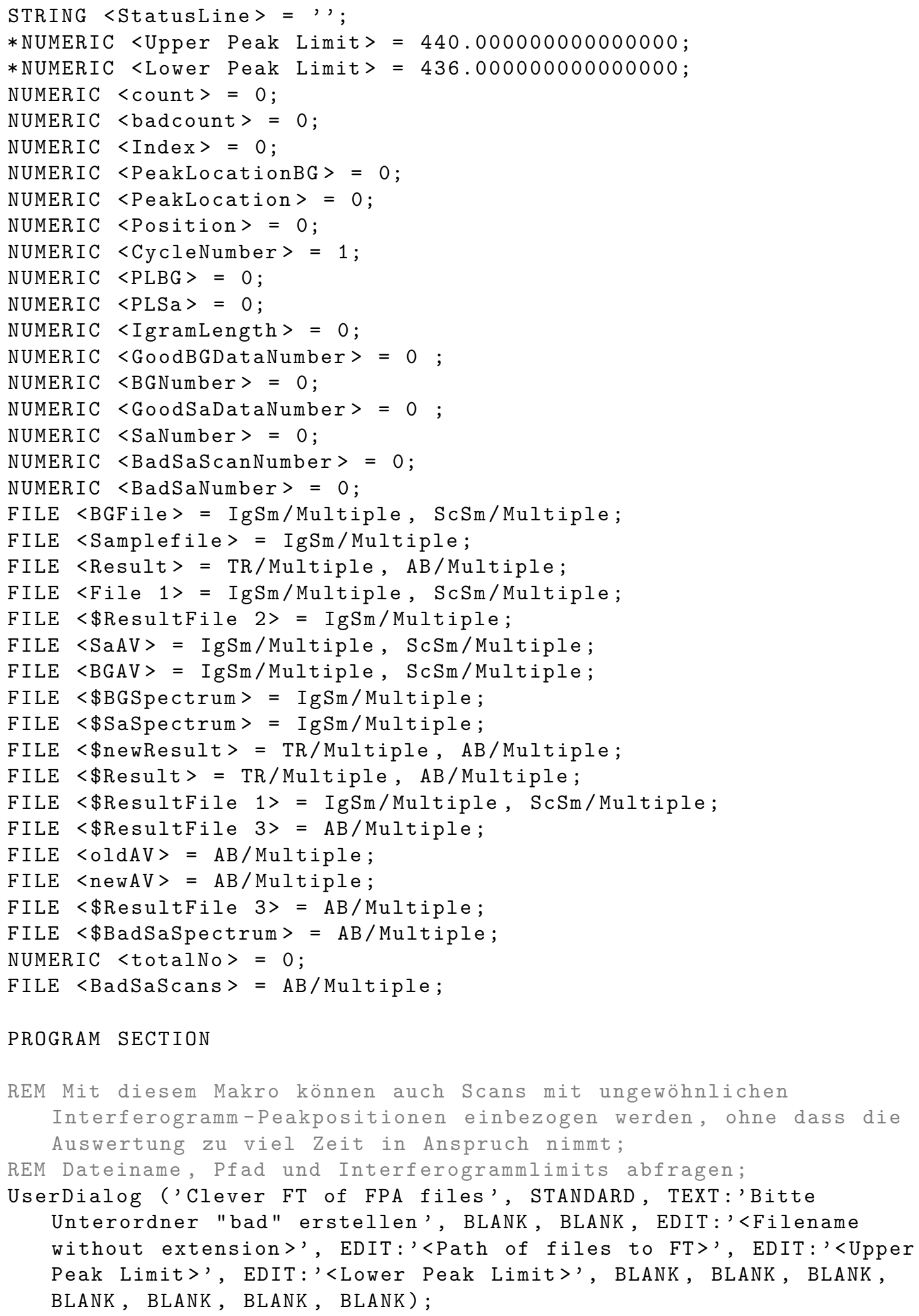


<StatusLine > = 'Starting Fourier Transformation Macro';

StaticMessage (SHOW, \{'<StatusLine $>$ ' $\}$ );

REM Hintergrunddateien einlesen und zählen;

<ListOfFilesnames $>=$ ScanPath ('<Path of files to FT $>\backslash$ Filename without extension $>$ BG*');

$\langle$ count $\rangle=$ GetArrayCount ( Listoffilesnames $>$ );

REM Dateinamen generieren;

<StatusLine > = 'Separating Scans with Bad Interferogram Positions';

StaticMessage (SHOW, \{'<StatusLine $>$ ' $\}$ );

StartLoop (<count $>, 0)$;

$\langle$ ActualBGFilename $>=$ '<ListOfFilesnames $>[\langle$ Index $>$ ' ;

REM Name des Probenscans generieren;

$\langle$ Position $>=$ FindString ('<ActualBGFilename >', '_BG.', NOCASE);

$<$ Part $1>=$ ' $<[0,<$ Position $>]$ ActualBGFilename $>$ ';

$\langle$ Position $\rangle=\langle$ Position $>+3$;

$\langle$ Part $2>=$ ' $<[<$ Position $>$, ] ActualBGFilename $>$ ';

$\langle$ ActualSampleFilename $\rangle=$ ' $\langle$ Part 1$\rangle\langle\text { Part } 2\rangle^{\prime}$;

REM Hintergrunddatei und Probendatei laden, Interferogrammpositionen einlesen;

[<BGFile $>$ :IgSm] = LoadFile ('<Path of files to FT $>\backslash<$ ActualBGFilename $>$ ', WARNING);

$\langle$ PeakLocationBG > GetParameter ( $[<B G F i l e>$ :IgSm/Multiple $]$, PKL);

[<Samplefile>:IgSm] = LoadFile ('<Path of files to FT $>\backslash<$ ActualSampleFilename $>$ ', WARNING);

<PeakLocation > = GetParameter ([<Samplefile>:IgSm/Multiple $]$, PKL);

REM Wenn die Peakposition für das BG-File nicht im vorgegebenen Rahmen liegt, werden BG und Sample Datei verschoben;

If (<PeakLocationBG>, .GT., <Upper Peak Limit >);

Rename ('<Path of files to FT> $<$ ActualBGFilename>', '<Path of files to FT $>\backslash$ bad $\backslash<$ ActualBGFilename $\rangle^{\prime}$ ) ;

Rename ('<Path of files to FT $><$ ActualSampleFilename $>$ ', '<Path of files to FT $>$ bad $\backslash<$ ActualSampleFilename $>^{\prime}$ );

Endif ();

If (<PeakLocationBG>, .LT., <Lower Peak Limit>);

Rename ('<Path of files to FT $>\backslash<$ ActualBGFilename $>$ ', '<Path of files to FT $>\backslash$ bad $\backslash<$ ActualBGFilename $\rangle^{\prime}$ ) ;

Rename ('<Path of files to FT $>\backslash<$ ActualSampleFilename $>$ ', '<Path of files to FT> $>$ bad $\backslash<$ ActualSampleFilename $>^{\prime}$ );

Endif ();

REM Ebenso für die Probenscans;

If (<PeakLocation>, .GT., <Upper Peak Limit>);

Rename ('<Path of files to FT $>\backslash<$ ActualBGFilename $>$ ', '<Path of files to FT $>\backslash$ bad $\backslash<$ ActualBGFilename $>^{\prime}$ );

Rename ('<Path of files to FT> \<ActualSamplefilename>', '<Path of files to FT $>\backslash$ bad $\backslash<$ ActualSampleFilename $>^{\prime}$ );

Endif ( ) ;

If (<PeakLocation>, . LT., <Lower Peak Limit>);

Rename ('<Path of files to FT $><$ ActualBGFilename $>$ ', '<Path of files to $F T>\backslash$ bad $\backslash<$ ActualBGFilename $>^{\prime}$ ) ; 
Rename ('<Path of files to FT $>\backslash<$ ActualSamplefilename $>$ ', '<Path of files to FT $>$ bad $\backslash<$ ActualSampleFilename $>^{\prime}$ );

Endif ();

Unload ( $[\langle$ BGFile $>],\{\})$;

Unload ( $[\langle$ Samplefile $\rangle],\{\})$;

$\langle$ Index $\rangle=\langle$ Index $\rangle+1$;

$\langle$ CycleNumber $\rangle=\langle$ CycleNumber $\rangle+1$;

EndLoop (0);

REM Interferogramm-Paare mit abweichenden Peakpositionen werden erst Fourier-transformiert und dann gemittelt;

$\langle$ StatusLine $\rangle=$ 'FT of Bad Scans';

StaticMessage (SHOW, \{'<StatusLine $>$ ' $\}$ );

$\langle$ Index $>=0$;

$\langle$ Position $>=0$;

LListOfFilesnames $>=$ ScanPath ('<Path of files to FT $>$ bad $\backslash<$ Filename without extension $>$ _BG*');

<badcount $>=$ GetArrayCount (<ListOfFilesnames $>$ );

StartLoop (<badcount $>, 1)$;

$\langle$ ActualBGFilename $>=$ ' $<$ ListOfFilesnames $>[\langle$ Index $>$ ]';

$\langle$ Position $>$ = FindString ('<ActualBGFilename>', '_BG.', NOCASE);

$\langle$ Part 1$\rangle=$ ' $<[0,<$ Position $>$ ActualBGFilename $>$ ';

$\langle$ Position $\rangle=\langle$ Position $\rangle+3$;

$\langle$ Part 2$\rangle=$ '<[<Position $>$, ] ActualBGFilename $>$ ';

$<$ ActualSampleFilename $>=$ ' $<$ Part $1><$ Part 2$\rangle^{\prime}$;

$[<\mathrm{BGFile}>: \mathrm{IgSm}]=$ LoadFile ('<Path of files to

FT $>\backslash$ bad $\backslash<$ ActualBGFilename $>$ ', WARNING);

LIgramLength $\rangle=$ GetParameter ([<BGFile >:IgSm/Multiple], NPT);

$\langle$ AquisitionMode $>$ = GetParameter ([<BGFile>:IgSm/Multiple $], A Q M)$;

$\langle$ PeakLocation $>$ = GetParameter ([<BGFile $>$ :IgSm/Multiple $], P K L)$;

FFT ( [<BGFile >:IgSm/Multiple], 0, \{FPP=<PeakLocation>, FSM='MA', $F S R=0, F S Y=128, F N E=0.000000, F N C=0.000000, F N L=0, F B W=3, F T T=0$, $\mathrm{FLR}=0, \mathrm{FHR}=32.000000, \mathrm{FTR}=1.000000, \mathrm{FTE}=50.000000$, $\left.\left.\mathrm{FTS}=3900.000000, \mathrm{FZF}={ }^{\prime} 2^{\prime}, \mathrm{FTA}={ }^{\prime} \mathrm{B} 3{ }^{\prime}, \mathrm{FHZ}={ }^{\prime} \mathrm{ML}{ }^{\prime}\right\}\right)$;

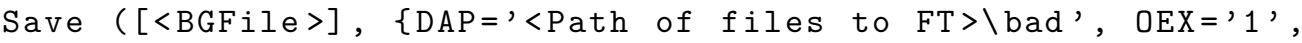
SAN ='<ActualBGFilename >', $C O F=130\})$;

[<Samplefile>:IgSm] = LoadFile ('<Path of files to FT $>\backslash$ bad $\backslash<$ ActualSampleFilename $>$ ', WARNING);

<IgramLength> = GetParameter ([<Samplefile>:IgSm/Multiple], NPT); $\langle$ AquisitionMode $\rangle=$ GetParameter ([<Samplefile>:IgSm/Multiple $], A Q M)$; <PeakLocation> = GetParameter ([<Samplefile>:IgSm/Multiple], PKL);

FFT ( [<Samplefile>:IgSm/Multiple], 0, \{FPP=<PeakLocation>, FSM='MA', $F S R=0, F S Y=128, F N E=0.000000, F N C=0.000000, F N L=0, F B W=3, F T T=0$, $F L R=0, F H R=32.000000, F T R=1.000000, F T E=50.000000$, $\left.\left.\mathrm{FTS}=3900.000000, \mathrm{FZF}={ }^{\prime} 2^{\prime}, \mathrm{FTA}={ }^{\prime} \mathrm{B} 3{ }^{\prime}, \mathrm{FHZ}={ }^{\prime} \mathrm{ML}{ }^{\prime}\right\}\right)$;

Save ( $[<$ Samplefile $>], \quad\left\{\mathrm{DAP}={ }^{\prime}<\right.$ Path of files to FT> $>$ bad', OEX='1', $\mathrm{SAN}=$ '<ActualSampleFilename $>$ ', $\mathrm{COF}=130\})$;

Calculate ([<Samplefile>:ScSm/Multiple][<BGFile>:ScSm/Multiple], $\{F O R=$ '[<Samplefile >:ScSm/Multiple]/[<BGFile >:ScSm/Multiple]' , $C D I=5248015\})$;

$\operatorname{ABTR}([<$ Samplefile >:TR/Multiple $],\{\mathrm{CCM}=3\})$; 
Save ( $[<$ Samplefile $>],\left\{\mathrm{DAP}=\right.$ '<Path of files to $\mathrm{FT}>\backslash \mathrm{bad},, \mathrm{PEX}={ }^{\prime}$ ', $\mathrm{SAN}=$ '<ActualSampleFilename $>$ ', $\mathrm{COF}=130\})$;

Unload ( $[\langle\mathrm{BGFile}\rangle],\{\})$;

Unload ([<Samplefile $>],\{\})$;

$\langle$ Index $\rangle=\langle$ Index $\rangle+1$;

EndLoop (1);

$\langle$ StatusLine $>=$ 'Averaging Bad Scans';

StaticMessage (SHOW, \{'<StatusLine $>$ ' $\}$ );

$\langle$ ListOfFilesnames $>=$ ScanPath (' $<$ Path of files to FT $>$ bad $\backslash<$ Filename without extension $>. *^{\prime}$ ) ;

$<$ BadSaScanNumber $>$ = GetArrayCount (<Listoffilesnames >);

$<$ BadSaScanNumber $>=<$ BadSaScanNumber $>-1$;

$<$ ResultFile 3$\rangle=$ Load $(0$, DAP $=$ '<Path of files to FT $>\backslash$ bad', $\mathrm{DAF}=$ '<ListOfFilesnames $>[0] '\})$;

StartLoop (<BadSaScanNumber $>, 2)$;

$\langle$ BadSaNumber $\rangle=\langle$ BadSaNumber $>+1$;

$\langle$ BadSaSpectrum $\rangle$ Load $(0,\{$ DAP $=$ ' $\langle$ Path of files to FT $>\backslash$ bad', DAF $=$ '<List0fFilesnames $\left.\left.>[<\text { BadSaNumber }>]^{\prime}\right\}\right)$;

$[<$ ResultFile 3>:AB/Multiple $]=$ Calculator ( $[<$ ResultFile

3>:AB/Multiple] [<\$BadSaSpectrum>:AB/Multiple],

$\{\mathrm{FOR}=$ ' $[<\$$ ResultFile

3>:AB/Multiple $\left.\left.]+[<\$ B \text { adSaSpectrum >:AB/Multiple }]^{\prime}\right\}\right)$;

EndLoop (2);

$\langle$ BadSaScanNumber $>=\langle$ BadSaScanNumber $>+1$;

[<\$ResultFile 3>:AB/Multiple $]=$ Calculator ( $[<$ ResultFile

3>:AB/Multiple $], \quad\{F O R='[<\$ R e s u l t F i l e$

3>:AB/Multiple]/<BadSaScanNumber $>$ '\});

SaveAs ( $[<\$ R e s u l t F i l e 3>], \quad\left\{\mathrm{DAP}={ }^{\prime}<\right.$ Path of files to FT $>\backslash$ bad', $0 E X=' 0^{\prime}$, $\mathrm{SAN}=$ '<Filename without extension >_AVold.<badcount>', COF=22\});

UnloadAll (0, \{\});

REM Bei 'normalen' Peakpositionen wird (aufgrund der Zeitersparnis) erst gemittelt und dann transformiert;

<StatusLine $>=$ 'Averaging Good BG Interferograms';

StaticMessage (SHOW, \{'<StatusLine $>$ ' $\}$ );

$<$ GoodBGDatalist $>$ ScanPath ('<Path of files to FT $>\langle$ Filename without extension >_BG*');

$<$ GoodBGDataNumber $>$ = GetArrayCount (<GoodBGDataList $>$ );

$\langle$ GoodBGDataNumber $>=\langle$ GoodBGDataNumber $>-1$;

$\langle$ ResultFile 1$\rangle=$ Load $(0,\{\mathrm{DAP}=$ ' $\langle$ Path of files to FT $>$ ', $\mathrm{DAF}=$ '<GoodBGDataList $>[0] '\})$;

StartLoop (<GoodBGDataNumber >, 3);

$\langle$ BGNumber $>=\langle$ BGNumber $>+1$;

$\langle \$ B G S p e c t r u m\rangle=$ Load $(0,\{D A P=$ ' $\langle$ Path of files to FT $>$ ', DAF $=$ ' $<$ GoodBGDataList $>[<B G N u m b e r>+1] '\})$;

[<\$ResultFile 1 >:IgSm/Multiple $]$ = Calculator ( $[<$ ResultFile 1>:IgSm/Multiple] [<\$BGSpectrum>:IgSm/Multiple], $\{\mathrm{FOR}=$ ' $[<\$$ ResultFile

$1>:$ IgSm/Multiple ] + [<\$BGSpectrum>: IgSm/Multiple ]'\});

EndLoop (3);

$\langle$ GoodBGDataNumber $>=\langle$ GoodBGDataNumber $>+1$; 


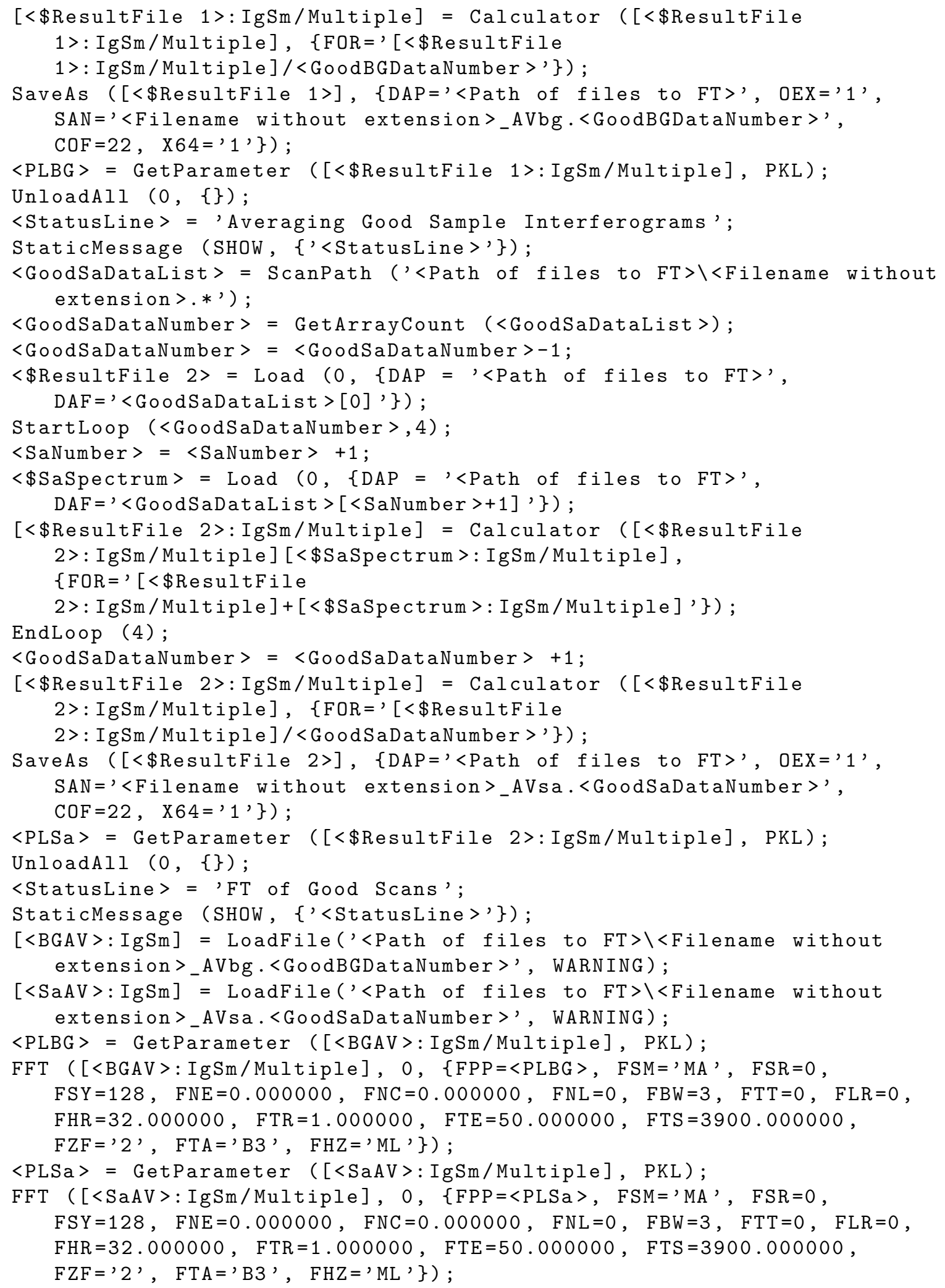




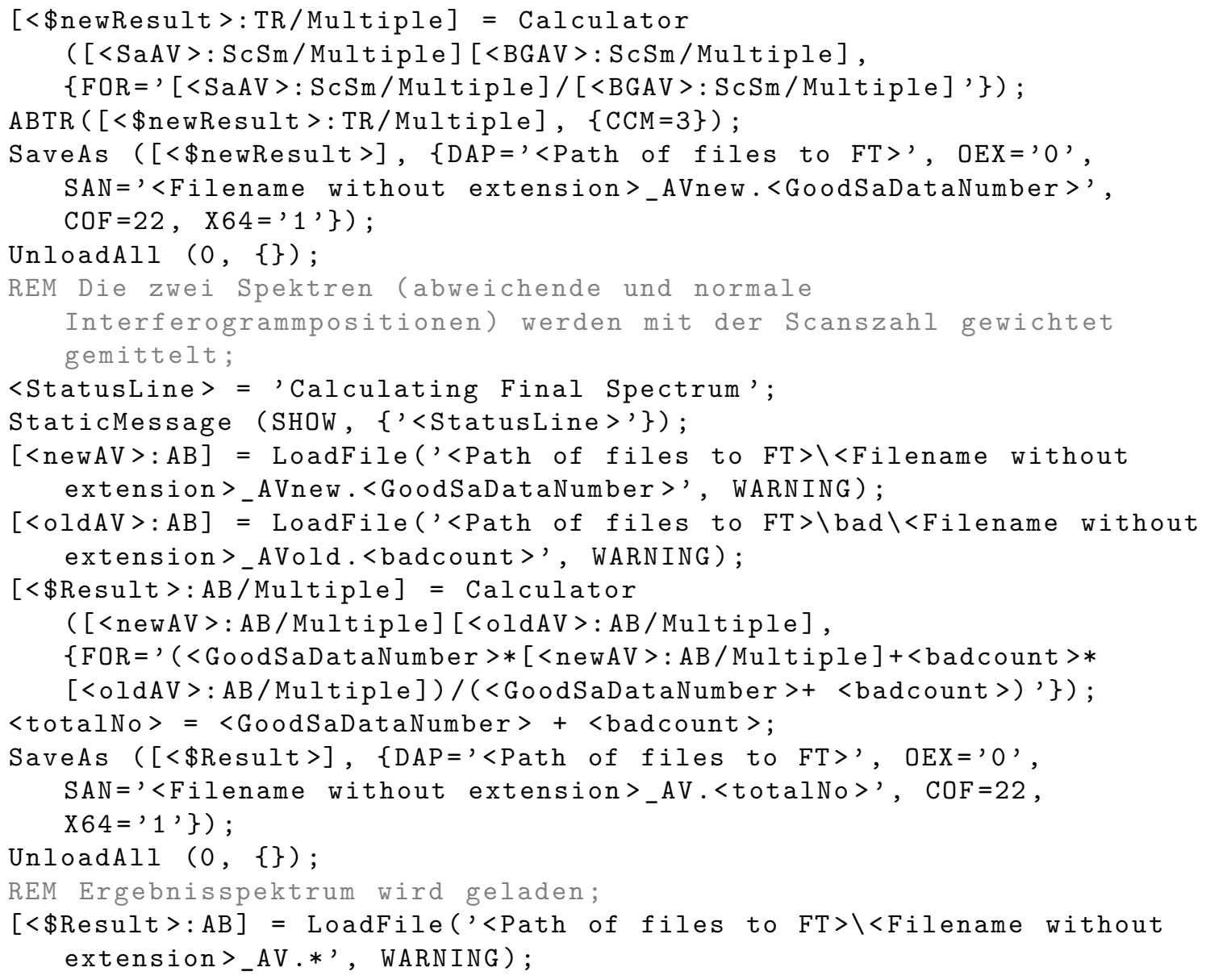




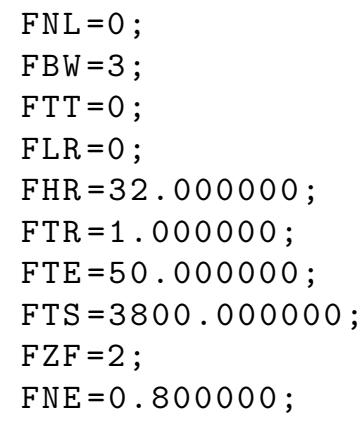

\section{Gauß-Binning-Makro}

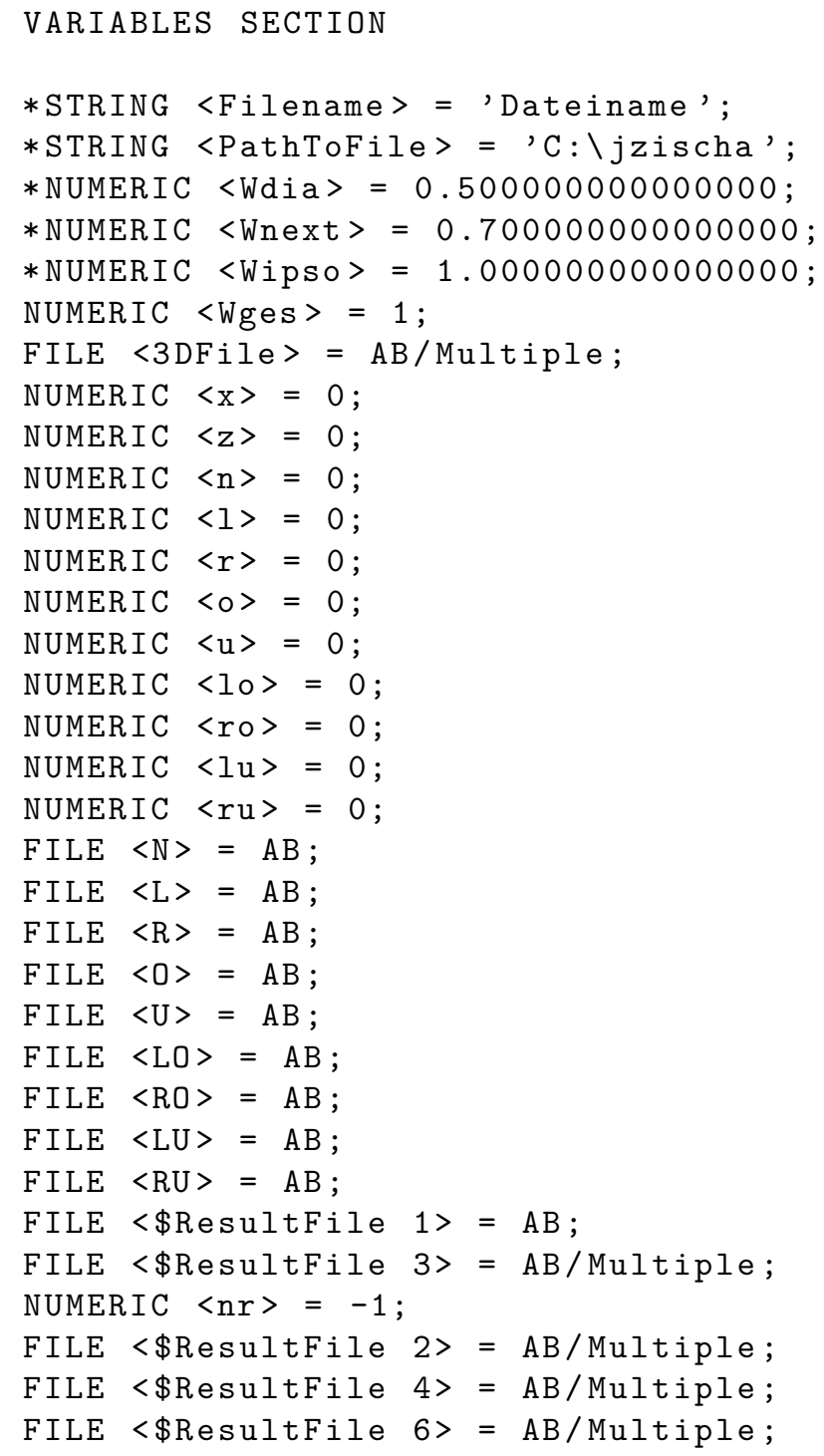


UserDialog ('Flexibles Datenbinning 32 x 32', STANDARD, EDIT:'<Filename>', EDIT:'<PathToFile>', BLANK, TEXT:'Gewichtung zentrales Spektrum', EDIT:'<Wipso>', BLANK, TEXT: 'Gewichtung Kantenspektren', EDIT:'<Wnext>', BLANK, TEXT:' Gewichtung Eckspektren', EDIT:'<Wdia>', BLANK, BLANK, BLANK);

REM Summe aus den Gewichtungen der Spektren;

$\langle$ Wges $\rangle=\langle$ Wipso $\rangle+4 *\langle$ Wnext $\rangle+4 *\langle$ Wdia $\rangle$;

REM Die Multiple-Datei wird in Einzelspektren zerlegt;

$[\langle 3 \mathrm{DFile}\rangle: \mathrm{AB}]=$ LoadFile ('<PathToFile $\rangle\langle\langle$ Filename $\rangle . *$ ', WARNING);

PostrunExtract ( $[\langle 3 \mathrm{DF} i l e\rangle: \mathrm{AB} / \mathrm{Multiple}],\{\mathrm{EXS}=1, \mathrm{EXE}=1, \mathrm{ECO}=0$,

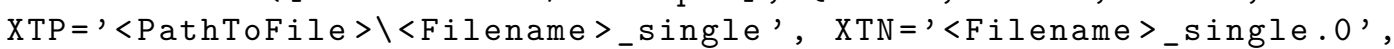
$\mathrm{XTI}=0, \mathrm{EAB}=0, \mathrm{ELF}=0, \mathrm{EUL}=0, \mathrm{EDM}=1\}$ );

REM Jedes nicht an der Kante liegende Spektrum wird mit seinen Nachbarn gewichtet gemittelt;

StartLoop (30, 0);

$\langle z\rangle=\langle z\rangle+1$;

StartLoop (30, 1);

$\langle\mathrm{x}\rangle=\langle\mathrm{x}\rangle+1$;

$\langle\mathrm{n} r\rangle=\langle\mathrm{n} r\rangle+1$;

$\langle\mathrm{n}\rangle=32 *\langle\mathrm{z}\rangle+\langle\mathrm{x}\rangle$;

$\langle 1\rangle=\langle\mathrm{n}\rangle+1$;

$\langle\mathrm{r}\rangle=\langle\mathrm{n}\rangle-1$;

$\langle 0\rangle=\langle\mathrm{n}\rangle-32$;

$\langle\mathrm{u}\rangle=\langle\mathrm{n}\rangle+32$

$\langle 10\rangle=\langle\mathrm{n}\rangle-31$;

$\langle$ ro $\rangle=\langle n\rangle-33$;

$\langle l u\rangle=\langle n\rangle+33$;

$\langle\mathrm{ru}\rangle=\langle\mathrm{n}\rangle+31$;

$[\langle\mathrm{N}\rangle: \mathrm{AB}]=$ LoadFile

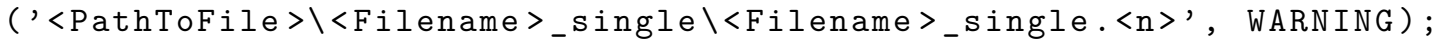

$[<\mathrm{R}\rangle: \mathrm{AB}]=$ LoadFile

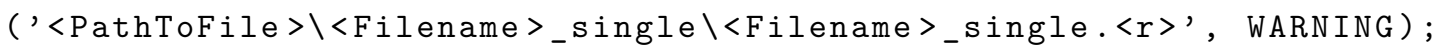

$[<\mathrm{L}\rangle: \mathrm{AB}]=$ LoadFile

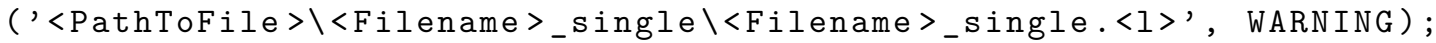
$[<0\rangle: \mathrm{AB}]=$ LoadFile

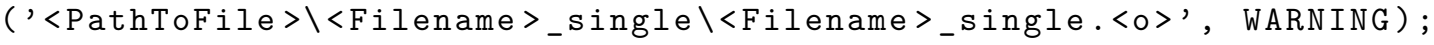

$[\langle\mathrm{U}\rangle: \mathrm{AB}]=$ LoadFile

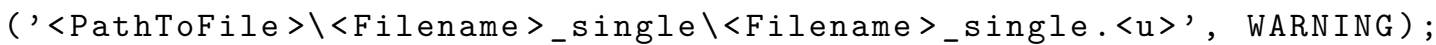

$[\langle\mathrm{LO}\rangle: \mathrm{AB}]=$ LoadFile

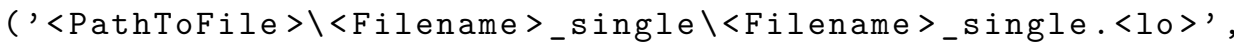
WARNING);

$[<R O\rangle: A B]=$ LoadFile

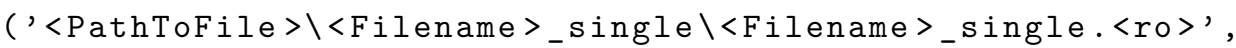
WARNING) ;

$[\langle\mathrm{LU}\rangle: \mathrm{AB}]=$ LoadFile

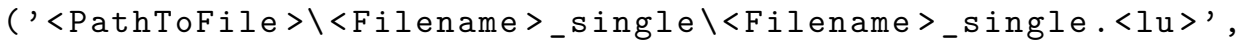
WARNING);

$[<\mathrm{RU}>: \mathrm{AB}]=$ LoadFile

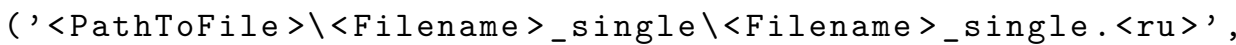
WARNING) ; 


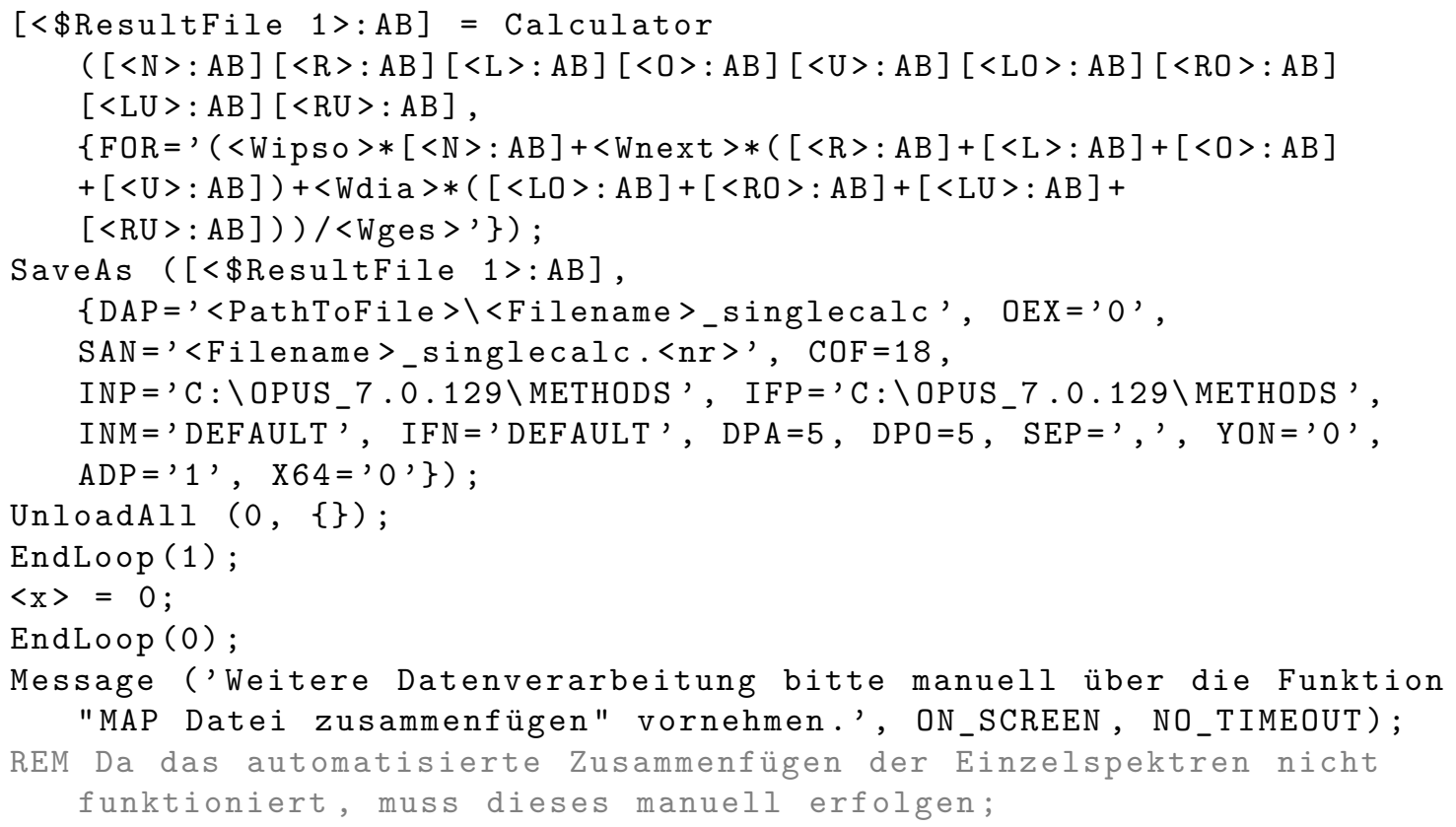

PARAMETER SECTION

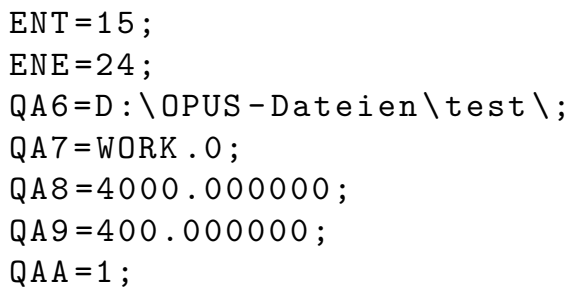




\section{Literaturverzeichnis}

[1] Y. Maréchal, The Hydrogen Bond and the Water Molecule: The physics and chemistry of water, aqueous and bio media Elsevier, Amsterdam, 2007.

[2] A. A. Vigasin, On the possibility to quantify contributions from true bound and metastable pairs to infrared absorption in pressurised water vapour, Mol. Phys. 2010, 108, 2309-2313.

[3] U. Buck, F. Huisken, Infrared spectroscopy of size-selected water and methanol clusters, Chem. Rev. 2000, 100, 3863-3890.

[4] F. N. Keutsch, R. J. Saykally, Water clusters: Untangling the mysteries of the liquid, one molecule at a time, Proceedings of the National Academy of Sciences 2001, 98, 10533-10540.

[5] K. E. Otto, Z. Xue, P. Zielke, M. A. Suhm, The Raman spectrum of isolated water clusters, Phys. Chem. Chem. Phys 2014, 16. Doi:10.1039/C3CP54272F.

[6] M. Y. Tretyakov, E. A. Serov, M. A. Koshelev, V. V. Parshin, A. F. Krupnov, Water dimer rotationally resolved millimeter-wave spectrum observation at room temperature, Phys. Rev. Lett. 2013, 110, 093001.

[7] W. Y. Wahlgren, G. Pál, J. Kardos, P. Porrogi, B. Szenthe, A. Patthy, L. Gráf, G. Katona, The catalytic aspartate is protonated in the Michaelis complex formed between trypsin and in vitro evolved substrate-like inhibitor, J. Biol. Chem. 2011, 286, 3587-3596.

[8] H.-S. Andrei, N. Solcà, O. Dopfer, Microhydration of protonated biomolecular building blocks: IR spectra of protonated imidazole-- ater $_{n}$ complexes, ChemPhysChem 2006, 7, 107-110.

[9] W. Massa, Kristallstrukturbestimmung, 5. Aufl. Teubner, Wiesbaden, 2007.

[10] T. Häber, U. Schmitt, M. A. Suhm, FTIR-spectroscopy of molecular clusters in pulsed supersonic slit-jet expansions, Phys. Chem. Chem. Phys. 1999, 1, 55735582.

[11] A. J. Stone, The Theory of Intermolecular Forces, International Series of Monographs on Chemistry, Bd. 32 Oxford University Press, Oxford, 1996. 
[12] F. London, The general theory of molecular forces, Trans. Faraday Soc. 1937, $33,8-26$.

[13] F. London, Zur Theorie und Systematik der Molekularkräfte, Z. Phys. 1930, 63, $245-279$.

[14] B. M. Axilrod, E. Teller, Interaction of the van der Waals type between three atoms, J. Chem. Phys. 1943, 11, 299-300.

[15] B. M. Axilrod, Triple-dipole interaction. I. Theory, J. Chem. Phys. 1951, 19, 719-724.

[16] L. Pauling, The nature of the chemical bond. Application of results obtained from the quantum mechanics and from a theory of paramagnetic susceptibility to the structure of molecules, J. Am. Chem. Soc. 1931, 53, 1367-1400.

[17] E. Arunan, G. R. Desiraju, R. A. Klein, J. Sadlej, S. Scheiner, I. Alkorta, D. C. Clary, R. H. Crabtree, J. J. Dannenberg, P. Hobza, H. G. Kjaergaard, A. C. Legon, B. Mennucci, D. J. Nesbitt, Definition of the hydrogen bond (IUPAC recommendations 2011), Pure Appl. Chem. 2011, 83, 1637-1641.

[18] P. A. Kollman, L. C. Allen, The theory of the hydrogen bond, Chem. Rev. 1972, 72, 283-303.

[19] J. Černý, P. Hobza, Non-covalent interactions in biomacromolecules, Phys. Chem. Chem. Phys. 2007, 9, 5291-5303.

[20] J. Joseph, E. D. Jemmis, Red-, blue-, or no-shift in hydrogen bonds: A unified explanation, J. Am. Chem. Soc. 2007, 129, 4620-4632.

[21] A. V. Iogansen, Direct proportionality of the hydrogen bonding energy and the intensification of the stretching $\nu(\mathrm{XH})$ vibration in infrared spectra, Spectrochim. Acta A 1999, 55, 1585-1612.

[22] A. Karpfen, Cooperative effects in hydrogen bonding, Adv. Chem. Phys. 2002, 123, 469-510.

[23] O. F. Hagena, Nucleation and growth of clusters in expanding nozzle flows, Surf. Sci. 1981, 106, 101-116.

[24] M. Herman, R. Georges, M. Hepp, D. Hurtmans, High resolution Fourier transform spectroscopy of jet-cooled molecules, Int. Rev. Phys. Chem. 2000, 19, 277325.

[25] A. E. Beylich, Struktur von Überschall-Freistrahlen aus Schlitzblenden, Z. Flugwiss. Weltraumforsch. 1979, 3, 48-58. 
[26] R. E. Smalley, L. Wharton, D. H. Levy, Molecular optical spectroscopy with supersonic beams and jets, Acc. Chem. Res. 1977, 10, 139-145.

[27] J. M. Hayes, Analytical spectroscopy in supersonic expansions, Chem. Rev. 1987, $87,745-760$.

[28] M. D. Morse, Supersonic beam sources, in: Experimental Methods in the Physical Sciences, Bd. 29B Academic Press, 1996, 21-47.

[29] S. DePaul, D. Pullman, B. Friedrich, A pocket model of seeded supersonic beams, J. Phys. Chem. 1993, 97, 2167-2171.

[30] C. Hartz, B. A. Wofford, R. F. Meads, R. R. Lucchese, J. W. Bevan, A nearinfrared Fourier-transform planar supersonic jet spectrometer for rovibrational studies of weakly bound dimers and trimers, Rev. Sci. Instrum. 1995, 66, 43754384 .

[31] M. Ito, Supersonic jet spectroscopy for the study of hydrogen bonding, J. Mol. Struct. 1988, 177, 173-190.

[32] D. J. Nesbitt, High-resolution, direct infrared laser absorption spectroscopy in slit supersonic jets: Intermolecular forces and unimolecular vibrational dynamics in clusters, Annu. Rev. Phys. Chem. 1994, 45, 367-399.

[33] D. Luckhaus, M. Quack, U. Schmitt, M. A. Suhm, On FTIR spectroscopy in asynchronously pulsed supersonic free jet expansions and on the interpretation of stretching spectra of HF clusters, Ber. Bunsenges. Phys. Chem. 1995, 99, 457-468.

[34] M. Quack, U. Schmitt, M. A. Suhm, Evidence for the $(\mathrm{HF})_{5}$ complex in the HF stretching FTIR absorption spectra of pulsed and continuous supersonic jet expansions of hydrogen fluoride, Chem. Phys. Lett. 1993, 208, 446-452.

[35] C. M. Lovejoy, D. J. Nesbitt, High sensitivity, high-resolution IR laser spectroscopy in slit supersonic jets: Application to $\mathrm{N}_{2} \mathrm{HF} \nu_{1}$ and $\nu_{5}+\nu_{1}-\nu_{5}$, J. Chem. Phys. 1987, 86, 3151-3165.

[36] A. Amirav, U. Even, J. Jortner, Absorption spectroscopy of ultracold large molecules in planar supersonic expansions, Chem. Phys. Lett. 1981, 83, 1-4.

[37] R. Georges, A. Bonnamy, A. Benidar, M. Decroi, J. Boissoles, FTIR free-jet setup for the high resolution spectroscopic investigation of condensable species, Mol. Phys. 2002, 100, 1551-1558.

[38] P. R. Griffiths, J. A. de Haseth, Fourier Transform Infrared Spectrometry, 2 Aufl. John Wiley \& Sons, Hoboken, 2007. 
[39] M. A. Suhm, F. Kollipost, Femtisecond single-mole infrared spectroscopy of molecular clusters, Phys. Chem. Chem. Phys. 2013, 15, 10702-10721.

[40] T. Di Paolo, C. Bourdéron, C. Sandorfy, Model calculations on the influence of mechanical and electrical anharmonicity on infrared intensities: Relation to hydrogen bonding, Can. J. Chem. 1972, 50, 3161-3166.

[41] T. Häber, U. Schmitt, C. Emmeluth, M. A. Suhm, Ragout-jet FTIR spectroscopy of cluster isomerism and cluster dynamics: from carboxylic acid dimers to $\mathrm{N}_{2} \mathrm{O}$ nanoparticles, Faraday Discuss. 2001, 118, 331-359.

[42] D. L. Snavely, S. D. Colson, K. B. Wiberg, Rotational cooling in a supersonic expansion of ammonia, J. Chem. Phys. 1981, 74, 6975-6976.

[43] A. Moudens, R. Georges, M. Goubet, J. Makarewicz, S. E. Lokshtanov, A. A. Vigasin, Direct absorption spectroscopy of water clusters formed in a continuous slit nozzle expansion, J. Chem. Phys. 2009, 131, 204312.

[44] B. E. Djakov, R. Enikov, D. Oliver, Supersonic jets with periodic structure due to arc plasma expansion into a low pressure ambient, Contrib. Plasma Phys. 1997, $37,57-76$.

[45] G. Dupeyrat, Progress in astronautics and aeronautics, in: Rarefied Gas Dynamics, 12th Symposium, Herausgeber S. S. Fisher, Bd. II, 74 1981, 812.

[46] B. Maté, I. A. Graur, T. Elizarova, I. Chirokov, G. Tejeda, J. M. Fernández, S. Montero, Experimental and numerical investigation of an axisymmetric supersonic jet, J. Fluid. Mech. 2001, 426, 177-197.

[47] A. Broc, S. de Benedictis, G. Dilecce, M. Vigliotti, R. G. Sharafutdinov, P. A. Skovorodko, Experimental and numerical investigation of an $\mathrm{O}_{2} / \mathrm{NO}$ supersonic free jet expansion, J. Fluid. Mech. 2004, 500, 211-237.

[48] M. Nishida, T. Nakagawa, H. Kobayashi, Density contours of a supersonic freejet, Exp. Fluids 1985, 3, 181-183.

[49] J. Panda, Shock oscillation in underexpanded screeching jets, J. Fluid. Mech. 1998, 363, 173-198.

[50] J. Panda, R. G. Seasholtz, Measurement of shock structure and shock-vortex interaction in underexpanded jets using Rayleigh scattering, Phys. Fluids 1999, 11, 3761-3777.

[51] B. André, T. Castelain, C. Bailly, Shock oscillations in a supersonic jet exhibiting antisymmetrical screech, AIAA Journal 2012, 50, 2017-2020. 
[52] C. K. W. Tam, Supersonic jet noise, Annu. Rev. Fluid Mech. 1995, 27, 17-43.

[53] D. C. Pack, On the formation of shock-waves in supersonic gas jets (twodimensional flow), Q. J. Mech. Appl. Math. 1948, 1, 1-17.

[54] E. V. Kuleznev, V. I. Baranov, D. N. Trubnikov, Numerical study of a free jet multinozzle source, Chem. Phys. Lett. 1994, 223, 255-262.

[55] T. Emilsson, T. D. Klots, R. S. Ruoff, H. S. Gutowsky, Rotational spectra and structures of the $\mathrm{OC}-$ and $\mathrm{H}_{3} \mathrm{~N}-\mathrm{HCN}-\mathrm{HF}$ trimers: Coaxial mixing nozzle for reactive species, J. Chem. Phys. 1990, 93, 6971-6976.

[56] M. Goubet, P. Asselin, P. Soulard, M. Lewerenz, Vibrational dynamics of medium strength hydrogen bonds: Fourier transform infrared spectra and band contour analysis of the DF stretching region of $\left(\mathrm{CH}_{2}\right)_{2} \mathrm{~S}-\mathrm{DF}, J$. Chem. Phys. 2004, 121, $7784-7794$.

[57] R. Georges, G. Durry, M. Bach, R. Pétrisse, R. Jost, M. Herman, Multinozzle supersonic expansion for Fourier transform absorption spectroscopy, Chem. Phys. Lett. 1995, 246, 601-606.

[58] Z. Xue, M. A. Suhm, Adding more weight to a molecular recognition unit: the low-frequency modes of carboxylic acid dimers, Mol. Phys. 2010, 108, 2279-2288.

[59] T. Scharge, C. Cézard, P. Zielke, A. Schütz, C. Emmeluth, M. A. Suhm, A peptide co-solvent under scrutiny: self-aggregation of 2,2,2-trifluoroethanol, Phys. Chem. Chem. Phys. 2007, 9, 4472-4490.

[60] T. Scharge, Schwache Bindungen mit starker Wirkung: Konformation und Aggregation von halogenierten Alkoholen, Dissertation, Georg-August-Universität Göttingen, 2007.

[61] T.-F. Hu, D. K. McLaughlin, Flow and acoustic properties of low Reynolds number underexpanded supersonic jets, J. Sound. Vib. 1990, 141, 485-505.

[62] A. Ben-Yakar, R. K. Hanson, Ultra-fast-framing schlieren system for studies of the time evolution of jets in supersonic crossflows, Exp. Fluids 2002, 32, 652-666.

[63] C. Huang, J. W. Gregory, J. P. Sullivan, A modified schlieren technique for micro flow visualization, Meas. Sci. Technol. 2007, 18, N32-N34.

[64] G. Raman, Cessation of screech in underexpanded jets, J. Fluid. Mech. 1997, 336, 69-90.

[65] G. Raman, Shock-induced flow resonance in supersonic jets of complex geometry, Phys. Fluids 1999, 11, 692-709. 
[66] R. S. Benson, D. E. Pool, Compressible flow through a two-dimensional slit, Int. J. Mech. Sci. 1965, 7, 315-336.

[67] C. Söller, R. Wenskus, P. Middendorf, G. E. A. Meier, F. Obermeier, Interferometric tomography for flow visualization of density fields in supersonic jets and convective flow, Appl. Opt. 1994, 33, 2921-2932.

[68] G. W. Faris, R. L. Byer, Quantitative optical tomographic imaging of a supersonic jet, Opt. Lett. 1986, 11, 413-415.

[69] G. Raman, Advances in understanding supersonic jet screech: review and perspective, Prog. Aerospace Sci. 1998, 34, 45-106.

[70] G. Tejeda, B. Maté, J. M. Fernández-Sánchez, S. Montero, Temperature and density mapping of supersonic jet expansions using linear Raman spectroscopy, Phys. Rev. Lett 1996, 76, 34-37.

[71] M. J. Thorpe, F. Adler, K. C. Cossel, M. H. G. de Miranda, J. Ye, Tomography of a supersonically cooled molecular jet using cavity-enhanced direct frequency comb spectroscopy, Chem. Phys. Lett. 2009, 468, 1-8.

[72] O. P. Postel, J. V. R. Heberlein, Two-dimensional optical emission imaging of a pulsed supersonic plasma jet, IEEE Trans. Plasma Sci. 1999, 27, 100-101.

[73] J. L. Palmer, R. K. Hanson, Temperature imaging in a supersonic free jet of combustion gases with two-line OH fluorescence, Appl. Opt. 1996, 35, 485-499.

[74] S. Montero, J. H. Morilla, G. Tejeda, J. M. Fernández, Experiments on small $\left(\mathrm{H}_{2}\right)_{\mathrm{n}}$ clusters, Eur. Phys. J. D 2009, 52, 31-34.

[75] B. Maté, G. Tejeda, S. Montero, Raman spectroscopy of supersonic jets of $\mathrm{CO}_{2}$ : Density, condensation, and translational, rotational, and vibrational temperatures, J. Chem. Phys. 1998, 108, 2676-2685.

[76] S. Montero, Temperature and entropy in supersonic free jets, Phys. Fluids 2013, 25, 056102 .

[77] A. Ramos, B. Maté, G. Tejeda, J. M. Fernández, S. Montero, Raman spectroscopy of hypersonic shock waves, Phys. Rev. E 2000, 62, 4940-4945.

[78] A. Ramos, J. M. Fernández, G. Tejeda, S. Montero, Quantitative study of cluster growth in free-jet expansions of $\mathrm{CO}_{2}$ by Rayleigh and Raman scattering, Phys. Rev. A 2005, 72, 053204.

[79] H.-Y. N. Holman, M. C. Martin, W. R. McKinney, Tracking chemical changes in a live cell: Biomedical applications of SR-FTIR spectromicroscopy, Spectroscopy 2003, 17, 139-159. 
[80] V. Sablinskas, G. Steiner, J. Ceponkus, R. Salzer, Infrared spectroscopic imaging with high spatial resolution and high sensitivity, in: Plasmonics in Biology and Medicine V, Proc. SPIE, Bd. 6869 2008, 68690X.

[81] I. W. Levin, R. Bhargava, Fourier transform infrared vibrational spectroscopic imaging: Integrating microscopy and molecular recognition, Annu. Rev. Phys. Chem. 2005, 56, 429-474.

[82] D. A. Scribner, M. R. Kruer, J. M. Killiany, Infrared focal plane array technology, Proc. IEEE 1991, 79, 66-85.

[83] S. G. Kazarian, K. L. A. Chan, Applications of ATR-FTIR spectroscopic imaging to biomedical samples, Biochim. Biophys. Acta 2006, 1758, 858-867.

[84] J. A. Dougan, S. G. Kazarian, Fourier transform infrared spectroscopic imaging of live cells, Spectrosc. Eur. 2013, 25, 6-12.

[85] E. N. Lewis, P. J. Treado, R. C. Reeder, G. M. Story, A. E. Dowrey, C. Marcott, I. W. Levin, Fourier transform spectroscopic imaging using an infrared focalplane array detector, Anal. Chem. 1995, 67, 3377-3381.

[86] C. M. Snively, J. L. Koenig, Characterizing the performance of a fast FT-IR imaging spectrometer, Appl. Spectrosc. 1999, 53, 170-177.

[87] C. M. Snively, S. Katzenberger, G. Oskarsdottir, J. Lauterbach, Fouriertransform infrared imaging using a rapid-scan spectrometer, Opt. Lett. 1999, 24, 1841-1843.

[88] J. Zischang, M. A. Suhm, Infrared absorption imaging of 2D supersonic jet expansions: Free expansion, cluster formation, and shock wave patterns, J. Chem. Phys. 2013, 139, 024201.

[89] L. J. Kozlowski, S. A. Cabelli, D. E. Cooper, K. Vural, Low background infrared hybrid focal plane array characterization, in: Infrared Detectors and Instrumentation, Proc. SPIE, Bd. 1946 1993, 199-213.

[90] J. F. Siliquini, L. Faraone, The vertical photoconductor: A novel device structure suitable for $\mathrm{HgCdTe}$ two-dimensional infrared focal plane arrays, Infrared Phys. Technol. 1997, 38, 205-221.

[91] T. Sprafke, J. W. Beletic, High-performance infrared focal plane arrays for space applications, Opt. Photonics News 2008, 19, 22-27.

[92] R. Bhargava, D. C. Fernandez, M. D. Schaeberle, I. W. Levin, Theory and application of gain ranging to Fourier transform infrared spectroscopic imaging, Appl. Spectrosc. 2001, 55, 1580-1589. 
[93] R. Bhargava, I. W. Levin, Effective time averaging of multiplexed measurements: A critical analysis, Anal. Chem. 2002, 74, 1429-1435.

[94] L. Shkedy, O. Amir, Z. Calahorra, J. Oiknine-Schlesinger, I. Szafranek, Temperature dependence of spatial noise in InSb focal plane arrays, in: Infrared Detectors and Focal Plane Arrays VI, Proc. SPIE, Bd. 4028 2000, 481-488.

[95] S. Tanimura, Y. Okada, K. Takeuchi, FTIR spectroscopy of $\mathrm{UF}_{6}$ clustering in a supersonic laval nozzle, J. Phys. Chem. 1996, 100, 2842-2848.

[96] G. Tejeda, F. Thibault, J. M. Fernández, S. Montero, Low-temperature inelastic collisions between hydrogen molecules and helium atoms, J. Chem. Phys. 2008, 128, 224308.

[97] E. R. T. Kerstel, K. K. Lehmann, B. H. Pate, G. Scoles, Reinvestigation of the acetylenic $\mathrm{C}-\mathrm{H}$ stretching fundamental of propyne via high resolution, optothermal spectroscopy: Nonresonant perturbations to $\nu_{1}, J$. Chem. Phys. 1994, 4 , $2588-2595$.

[98] G. Herzberg, Molecular spectra and molecular structure. I. Spectra of diatomic molecules, 2 Aufl. Van Nostrand, 1950.

[99] R. Georges, M. Bach, M. Herman, High resolution FTIR spectroscopy using a slit jet: sampling the overtone spectrum of ${ }^{12} \mathrm{C}_{2} \mathrm{H}_{4}$, Mol. Phys. 1997, 90, 381-387.

[100] J. M. Hollas, Modern Spectroscopy, 4. Aufl. John Wiley \& Sons, Chichester, 2004.

[101] R. D. Oparin, M. V. Fedotova, Influence of isobaric heating of hydrogen bonding in precritical water, Russ. J. Gen. Chem. 2007, 77, 17-24.

[102] J. N. Finch, E. R. Lippincott, Hydrogen bond systems: Temperature dependence of $\mathrm{OH}$ frequency shifts and $\mathrm{OH}$ band intensities, J. Chem. Phys. 1956, 24, 908909 .

[103] A. Ramos, G. Tejeda, J. M. Fernández, S. Montero, Nonequilibrium processes in supersonic jets of $\mathrm{N}_{2}, \mathrm{H}_{2}$, and $\mathrm{N}_{2}+\mathrm{H}_{2}$ mixtures: (I) Zone of silence, J. Phys. Chem. A 2009, 113, 8506-8512.

[104] C. Emmeluth, V. Dyczmons, M. A. Suhm, Tuning the hydrogen bond donor/acceptor isomerism in jet-cooled mixed dimers of aliphatic alcohols, J. Phys. Chem. A 2006, 110, 2906-2915.

[105] R. W. Larsen, P. Zielke, M. A. Suhm, Hydrogen-bonded OH stretching modes of methanol clusters: A combined IR and Raman isotopomer study, J. Chem. Phys. 2007, 126, 194307. 
[106] A. Bizzarri, S. Stolte, J. Reuss, J. G. C. M. van Duijneveldt-van de Rijdt, F. B. van Duijneveldt, Infrared excitation and dissociation of methanol dimers and trimers, Chem. Phys. 1990, 143, 423-435.

[107] D. J. Nesbitt, T. Häber, M. A. Suhm, General discussion, Faraday Discuss. 2001, $118,305-310$.

[108] C. Cézard, C. A. Rice, M. A. Suhm, OH-stretching red shifts in bulky hydrogenbonded alcohols: Jet spectroscopy and modeling, J. Phys. Chem. A 2006, 110, 9839-9848.

[109] C. A. Rice, Jet-FTIR Spectroscopy of Biomolecular Model Systems, Dissertation, Georg-August-Universität Göttingen, 2007.

[110] M. Albrecht, Biologisch relevante Wasserstoffbrückensysteme im Überschallstrahl: Steuerung der Aggregation durch Substitution, Dissertation, Georg-AugustUniversität Göttingen, 2009.

[111] J. M. Flaud, C. Camy-Peyret, Vibration-rotation intensities in $\mathrm{H}_{2}$ O-type molecules: Application to the $2 \nu_{2}, \nu_{1}$, and $\nu_{3}$ bands of $\mathrm{H}_{2}^{16} \mathrm{O}$, J. Mol. Spectrosc. 1975, $55,278-310$.

[112] J. M. Flaud, C. Camy-Peyret, The interacting states (020), (100), and (001) of $\mathrm{H}_{2}^{16} \mathrm{O}$, J. Mol. Spectrosc. 1974, 51, 142-150.

[113] W. O. George, B. F. Jones, R. H. Lewis, Water and its homologues: a comparison of hydrogen-bonding phenomena, Phil. Trans. R. Soc. Lond. A 2001, 359, 16111629 .

[114] M. A. Suhm, Hydrogen bond dynamics in alcohol clusters, Adv. Chem. Phys. 2009, 142, 1-57.

[115] D. Zimmermann, T. Häber, H. Schaal, M. A. Suhm, Hydrogen bonded rings, chains and lassos: the case of t-butyl alcohol clusters, Mol. Phys. 2001, 99, 413425.

[116] U. Buck, I. Ettischer, Vibrational predissociation spectra of size selected methanol clusters: New experimental results, J. Chem. Phys. 1998, 108, 33-38.

[117] M. J. Frisch, G. W. Trucks, H. B. Schlegel, G. E. Scuseria, M. A. Robb, J. R. Cheeseman, G. Scalmani, V. Barone, B. Mennucci, G. A. Petersson, H. Nakatsuji, M. Caricato, X. Li, H. P. Hratchian, A. F. Izmaylov, J. Bloino, G. Zheng, J. L. Sonnenberg, M. Hada, M. Ehara, K. Toyota, R. Fukuda, J. Hasegawa, M. Ishida, T. Nakajima, Y. Honda, O. Kitao, H. Nakai, T. Vreven, J. A. Montgomery, 
Jr., J. E. Peralta, F. Ogliaro, M. Bearpark, J. J. Heyd, E. Brothers, K. N. Kudin, V. N. Staroverov, R. Kobayashi, J. Normand, K. Raghavachari, A. Rendell, J. C. Burant, S. S. Iyengar, J. Tomasi, M. Cossi, N. Rega, J. M. Millam, M. Klene, J. E. Knox, J. B. Cross, V. Bakken, C. Adamo, J. Jaramillo, R. Gomperts, R. E. Stratmann, O. Yazyev, R. C. A. J. Austi and, C. Pomelli, J. W. Ochterski, R. L. Martin, K. Morokuma, V. G. Zakrzewski, G. A. Voth, P. Salvador, J. J. Dannenberg, S. Dapprich, A. D. Daniels, O. Farkas, J. B. Foresman, J. V. Ortiz, J. Cioslowski, D. J. Fox, Gaussian 09, Revision A.02, Gaussian, Inc., Wallingford CT, 2009.

[118] R. W. Larsen, Persönliche Mitteilungen.

[119] J. Gräfenstein, D. Cremer, An efficient algorithm for the density-functional theory treatment of dispersion interactions, J. Chem. Phys. 2009, 130, 124105.

[120] S. Abraham, Schwingungsspektroskopie nah- und überkritischer Lösungsmittel, Dissertation, Georg-August-Universität Göttingen, 2013.

[121] J. Zischang, J. J. Lee, M. A. Suhm, Where does the first water molecule go in imidazole?, J. Chem. Phys. 2011, 135, 061102.

[122] M. Koné, B. Illien, C. Laurence, J. Graton, Can quantum-mechanical calculations yield reasonable estimates of hydrogen-bonding acceptor strength? The case of hydrogen-bonded complexes of methanol, J. Phys. Chem. A 2011, 115, 1397513985.

[123] R. Ludwig, Wasser: von Clustern in die Flüssigkeit, Angew. Chem. 2001, 113, $1856-1876$.

[124] L. A. Curtiss, M. Blander, Thermodynamic properties of gas-phase hydrogenbonded complexes, Chem. Rev. 1988, 88, 827-841.

[125] Y. Scribano, N. Goldman, R. J. Saykally, C. Leforestier, Water dimers in the atmosphere III: Equilibrium constant from a flexible potential, J. Phys. Chem. A 2006, 110, 5411-5419.

[126] Y. J. Kim, B. E. Wyslouzil, G. Wilemski, J. Wölk, R. Strey, Isothermal nucleation rates in supersonic nozzles and the properties of small water clusters, J. Phys. Chem. A 2004, 108, 4365-4377.

[127] M. A. Suhm, HF vapor, Ber. Bunsenges. Phys. Chem. 1995, 99, 1159-1167.

[128] Y. Maréchal, IR spectra of carboxylic adics in the gas phase: A quantitative reinvestigation, J. Chem. Phys. 1987, 87, 6344-6353. 
[129] E. K. Plyler, W. W. Sleator, Further study of the absorption of infrared radiation by water vapor, Phys. Rev. 1931, 37, 1493-1507.

[130] S. Wuelfert, D. Herren, S. Leutwyler, Supersonic jet CARS spectra of small water clusters, J. Chem. Phys. 1987, 86, 3751-3753.

[131] G. T. Fraser, R. D. Suenram, L. H. Coudert, Microwave electric-resonance optothermal spectroscopy of $\left(\mathrm{H}_{2} \mathrm{O}\right)_{2}$, J. Chem. Phys. 1989, 90, 6077-6085.

[132] D. F. Coker, R. E. Miller, R. O. Watts, The infrared predissociation spectra of water clusters, J. Chem. Phys. 1985, 82, 3554-3562.

[133] J. B. Paul, C. P. Collier, R. J. Saykally, J. J. Scherer, A. O'Keefe, Direct measurement of water cluster concentrations by infrared cavity ringdown laser absorption spectroscopy, J. Phys. Chem. A 1997, 101, 5211-5214.

[134] D. J. Paynter, I. V. Ptashnik, K. P. Shine, K. M. Smith, Pure water vapor continuum measurements between 3100 and $4400 \mathrm{~cm}^{-1}$ : Evidence for water dimer absorption in near atmospheric conditions, Geophys. Res. Lett. 2007, 34, L12808.

[135] I. V. Ptashnik, K. P. Shine, A. A. Vigasin, Water vapour self-continuum and water dimers: 1. Analysis of recent work, J. Quant. Spectrosc. Radiat. Transfer 2011, 112, 1286-1303.

[136] H. R. Carlon, Variation in emission spectra from warm water fogs: evidence for clusters in the vapor phase, Infrared Phys. 1979, 19, 49-64.

[137] A. J. L. Shillings, S. M. Ball, M. J. Barber, J. Tennyson, R. L. Jones, An upper limit for water dimer absorption in the $750 \mathrm{~nm}$ spectral region and a revised water line list, Atmos. Chem. Phys. 2011, 11, 4273-4287.

[138] I. V. Ptashnik, R. A. McPheat, K. P. Shine, K. M. Smith, R. G. Williams, Water vapor self-continuum absorption in near-infrared windows derived from laboratory measurements, J. Geophys. Res. 2011, 116, D16305.

[139] I. V. Ptashnik, R. A. McPheat, K. P. Shine, K. M. Smith, R. G. Williams, Water vapour foreign-continuum absorption in near-infrared windows from laboratory measurements, Phil. Trans. R. Soc. A 2012, 370, 2557-2577.

[140] A. A. Vigasin, A. I. Pavlyuchko, Y. Jin, S. Ikawa, Density evolution of absorption bandshapes in the water vapor $\mathrm{OH}$-stretching fundamental and overtone: evidence for molecular aggregation, J. Mol. Struct. 2005, 742, 173-181.

[141] A. Borner, Z. Li, D. A. Levin, Development of a water cluster evaporation model using molecular dynamics, AIP Conf. Proc. 2011, 1333, 847-852.

\section{XXXVIII}


[142] W. S. Benedict, N. Gailar, E. K. Plyler, Rotation-vibration spectra of deuterated water vapor, J. Chem. Phys. 1956, 24, 1139-1165.

[143] C. Camy-Peyret, J.-M. Flaud, Line positions and intensities in the $\nu_{2}$ band of $\mathrm{H}_{2}^{16} \mathrm{O}$, Mol. Phys. 1976, 32, 523-537.

[144] J. E. Bertie, M. K. Ahmed, H. H. Eysel, Infrared intensities of liquids. 5. Optical and dielectric constants, integrated intensities, and dipole moment derivatives of $\mathrm{H}_{2} \mathrm{O}$ and $\mathrm{D}_{2} \mathrm{O}$ at $22^{\circ} \mathrm{C}$, J. Phys. Chem. 1989, 93, 2210-2218.

[145] V. Babin, F. Paesani, The curious case of the water hexamer: Cage vs. prism, Chem. Phys. Lett. 2013, 580, 1-8.

[146] C. J. Tainter, J. L. Skinner, The water hexamer: Three-body interactions, structures, energetics, and $\mathrm{OH}$-stretch spectroscopy at finite temperature, J. Chem. Phys. 2012, 137, 104304.

[147] C. Camy-Peyret, J. M. Flaud, G. Guelachvili, C. Amiot, High resolution Fourier transform spectrum of water between 2930 and $4255 \mathrm{~cm}^{-1}$, Mol. Phys. 1973, 26, $825-855$.

[148] D. R. Lide (Herausgeber), Handbook of Chemistry and Physics, 82. Aufl. CRC Press, Boca Raton, 2001.

[149] C. M. Western, pgopher, a Program for Simulating Rotational Structure, University of Bristol, 2013. URL http://pgopher.chm.bris.ac.uk, Version 8.0.102.

[150] G. J. Maclay, Integrated absorptances of spectral line groups in the 2.7- $\mu$ bands of hot water vapor, including effects of centrifugal distortion, J. Chem. Phys. 1965, 43, 185-191.

[151] C. Manca Tanner, M. Quack, D. Schmidiger, Nuclear spin symmetry conservation and relaxation in water $\left({ }^{1} \mathrm{H}_{2}^{16} \mathrm{O}\right)$ studied by cavity ring-down (CRD) spectroscopy of supersonic jets, J. Phys. Chem. A 2013, 117, 10105-10118.

[152] J. K. Messer, F. C. De Lucia, P. Helminger, The pure rotational spectrum of water vapor - a millimeter, submillimeter, and far infrared analysis, Int. J. Infrared Millim. Waves 1983, 4, 505-539.

[153] A. E. K. Sundén, K. Støchkel, S. Panja, U. Kadhane, P. Hvelplund, S. Brønsted Nielsen, H. Zettergren, B. Dynefors, K. Hansen, Heat capacities of freely evaporating charged water clusters, J. Chem. Phys. 2009, 130, 224308.

[154] J. M. Soler, N. García, Nonequilibrium internal and translational temperature of clusters in homogeneous nucleation, Phys. Rev. A 1983, 27, 3300-3306. 
[155] J. Brudermann, U. Buck, V. Buch, Isomerization and melting-like transition of size-selected water nonamers, J. Phys. Chem. A 2002, 106, 453-457.

[156] A. L. Garden, L. Halonen, H. G. Kjaergaard, Widening of the hydrogen bonded $\mathrm{OH}$-stretching bands due to the wagging and OO-stretching modes in $\mathrm{H}_{2} \mathrm{O} \cdot \mathrm{H}_{2} \mathrm{O}$, Chem. Phys. Lett. 2011, 513, 167-172.

[157] F. N. Keutsch, R. S. Fellers, M. G. Brown, M. R. Viant, P. B. Petersen, R. J. Saykally, Hydrogen bond breaking dynamics of the water trimer in the translational and librational band region of liquid water, J. Am. Chem. Soc. 2001, 123, $5938-5941$.

[158] B. Tremblay, B. Madebène, M. E. Alikhani, J. P. Perchard, The vibrational spectrum of the water trimer: Comparison between anharmonic ab initio calculations and neon matrix infrared data between 11,000 and $90 \mathrm{~cm}^{-1}$, Chem. Phys. 2010, 378, 27-36.

[159] A. K. Samanta, L. C. Ch'ng, H. Reisler, Imaging bond breaking and vibrational energy transfer in small water containing clusters, Chem. Phys. Lett. 2013, 575, $1-11$.

[160] T. A. Odintsova, M. Y. Tretyakov, Evidence of true bound and metastable dimers and trimers presence in high temperature water vapor spectra, J. Quant. Spectrosc. Radiat. Transfer 2013, 120, 134-137.

[161] M. N. Slipchenko, K. E. Kuyanov, B. G. Sartakov, A. F. Vilesov, Infrared intensity in small ammonia and water clusters, J. Chem. Phys. 2006, 124, 241101.

[162] S. A. Clough, Y. Beers, G. P. Klein, L. S. Rothman, Dipole moment of water from Stark measurements of $\mathrm{H}_{2} \mathrm{O}, \mathrm{HDO}$, and $\mathrm{D}_{2} \mathrm{O}$, J. Chem. Phys. 1973, 59, $2254-2259$.

[163] K. Kuyanov-Prozument, M. Y. Choi, A. F. Vilesov, Spectrum and infrared intensities of OH-stretching bands of water dimers, J. Chem. Phys. 2010, 132, 014304 .

[164] K. Liu, R. S. Fellers, M. R. Viant, R. P. McLaughlin, M. G. Brown, R. J. Saykally, A long path length pulsed slit valve appropriate for high temperature operation: Infrared spectroscopy of jet-cooled large water clusters and nucleotide bases, Rev. Sci. Instrum. 1996, 67, 410-416.

[165] M. Losada, S. Leutwyler, Water hexamer clusters: Structures, energies, and predicted mid-infrared spectra, J. Chem. Phys. 2002, 117, 2003-2016. 
[166] D. Skvortsov, S. J. Lee, M. Y. Choi, A. F. Vilesov, Hydrated HCl clusters, $\mathrm{HCl}\left(\mathrm{H}_{2} \mathrm{O}\right)_{1-3}$, in helium nanodroplets: Studies of free $\mathrm{OH}$ vibrational stretching modes, J. Phys. Chem. A 2009, 113, 7360-7365.

[167] M. L. Antipova, D. L. Gurina, V. E. Petrenko, The structure of H-bonded clusters in sub- and supercritical water, Russ. J. Phys. Chem. A 2011, 85, 977-982.

[168] M. L. Antipova, D. L. Gurina, V. E. Petrenko, Structure of hydrogen-bonded associates in supercritical water under low and high pressures, Russ. J. Phys. Chem. A 2013, 87, 449-453.

[169] J. P. Beck, J. M. Lisy, Infrared spectroscopy of hydrated alkali metal cations: Evidence of multiple photon absorption, J. Chem. Phys. 2011, 135, 044302.

[170] J. S. Prell, E. R. Williams, Structures of thermal, mass-selected water clusters probed with hydrophobic ion tags and infrared photodissociation spectroscopy, J. Am. Chem. Soc. 2009, 131, 4110-4119.

[171] A. W. Garrett, D. L. Severance, T. S. Zwier, Multiphoton ionization studies of $\mathrm{C}_{6} \mathrm{H}_{6}-\left(\mathrm{CH}_{3} \mathrm{OH}\right)_{n}$ clusters. I. Comparisons with $\mathrm{C}_{6} \mathrm{H}_{6}-\left(\mathrm{H}_{2} \mathrm{O}\right)_{n}$ clusters, J. Chem. Phys. 1992, 96, 7245-7258.

[172] R. N. Pribble, T. S. Zwier, Size-specific infrared spectra of benzene- $\left(\mathrm{H}_{2} \mathrm{O}\right)_{n}$ clusters $(n=1$ through 7$)$ : Evidence for noncyclic $\left(\mathrm{H}_{2} \mathrm{O}\right)_{n}$ structures, Science 1994, $265,75-79$.

[173] R. Parthasarathi, M. Elango, V. Subramanian, N. Sathyamurthy, Structure and stability of water chains $\left(\mathrm{H}_{2} \mathrm{O}\right)_{n}, n=5-20$, J. Phys. Chem. A 2009, 113, $3744-3749$.

[174] P. Bordat, D. Bégué, R. Brown, A. Marbeuf, H. Cardy, I. Baraille, The IR spectrum of supercritical water: Combined molecular dynamics/quantum mechanics strategy and force field for cluster sampling, Int. J. Quantum Chem. 2012, 112, $2578-2584$.

[175] A. G. Kalinichev, S. V. Churakov, Size and topology of molecular clusters in supercritical water: a molecular dynamics simulation, Chem. Phys. Lett. 1999, 302, 411-417.

[176] A. G. Kalinichev, S. V. Churakov, Thermodynamics and structure of molecular clusters in supercritical water, Fluid Phase Equilib. 2001, 183-184, 271-278.

[177] Y. C. Choi, C. Pak, K. S. Kim, Electric field effects on water clusters $(n=$ $3-5)$ : Systematic ab initio study of structures, energetics, and transition states, J. Chem. Phys. 2006, 124, 094308. 
[178] Y. Maréchal, Infrared spectra of a poorly known species: Water. 3, J. Phys. Chem. 1993, 97, 2846-2850.

[179] A. Engdahl, B. Nelander, Water in krypton matrices, J. Mol. Struct. 1989, 193, 101-109.

[180] L. M. Goss, S. W. Sharpe, T. A. Blake, V. Vaida, J. W. Brault, Direct absorption spectroscopy of water clusters, J. Phys. Chem. A 1999, 103, 8620-8624.

[181] J. B. Paul, R. A. Provencal, C. Chapo, K. Roth, R. Casaes, R. J. Saykally, Infrared cavity ringdown spectroscopy of the water cluster bending vibrations, J. Phys. Chem. A 1999, 103, 2972-2974.

[182] W. Klopper, M. Quack, M. A. Suhm, Explicitly correlated coupled cluster calculations of the dissociation energies and barriers to concerted hydrogen exchange of $(\mathrm{HF})_{n}$ oligomers $(n=2,3,4,5)$, Mol. Phys. 1998, 94, 105-119.

[183] M. R. Viant, J. D. Cruzan, D. D. Lucas, M. G. Brown, K. Liu, R. J. Saykally, Pseudorotation in water trimer isotopomers using Terahertz laser spectroscopy, J. Phys. Chem. A 1997, 101, 9032-9041.

[184] K. Liu, M. G. Brown, M. R. Viant, J. D. Cruzan, R. J. Saykally, Far-infrared VRT spectroscopy of two water trimer isotopomers: vibrationally averaged structures and rearrangement dynamics, Mol. Phys. 1996, 89, 1373-1396.

[185] J. Ceponkus, P. Uvdal, B. Nelander, Water tetramer, pentamer, and hexamer in inert matrices, J. Phys. Chem. A 2012, 116, 4842-4850.

[186] J. B. Paul, R. A. Provencal, C. Chapo, A. Petterson, R. J. Saykally, Infrared cavity ringdown spectroscopy of water clusters: O-D stretching bands, J. Chem. Phys. 1998, 109, 10201-10206.

[187] K. Liu, M. G. Brown, C. Carter, R. J. Saykally, J. K. Gregory, D. C. Clary, Characterization of a cage form of the water hexamer, Nature 1996, 381, 501503.

[188] F. Huisken, M. Kaloudis, A. Kulcke, Infrared spectroscopy of small size-selected water clusters, J. Chem. Phys. 1996, 104, 17-25.

[189] M. Quack, J. Stohner, M. A. Suhm, Analytical three-body interaction potentials and hydrogen bond dynamics of hydrogen fluoride aggregates, $(\mathrm{HF})_{n}, n \geq 3$, J. Mol. Struct. 2001, 599, 381-425.

[190] D. W. Michael, J. M. Lisy, Vibrational predissociation spectroscopy of $(\mathrm{HF})_{3}$, J. Chem. Phys. 1986, 85, 2528-2537. 
[191] A. Engdahl, B. Nelander, On the structure of the water trimer. A matrix isolation study, J. Chem. Phys. 1987, 86, 4831-4837.

[192] F. N. Keutsch, J. D. Cruzan, R. J. Saykally, The water trimer, Chem. Rev. 2003, 103, 2533-2577.

[193] S. S. Xantheas, Cooperativity and hydrogen bonding network in water clusters, Chem. Phys. 2000, 258, 225-231.

[194] E. G. Robertson, J. P. Simons, Getting into shape: Conformational and supramolecular landscapes in small biomolecules and their hydrated clusters, Phys. Chem. Chem. Phys. 2001, 3, 1-18.

[195] A. A. Adesokan, G. M. Chaban, O. Dopfer, R. B. Gerber, Vibrational spectroscopy of protonated imidazole and its complexes with water molecules: Ab initio anharmonic calculations and experiments, J. Phys. Chem. A 2007, 111, 73747381.

[196] G. J. Bartlett, C. T. Porter, N. Borkakoti, J. M. Thornton, Analysis of catalytic residues in enzyme active sites, J. Mol. Biol. 2002, 324, 105-121.

[197] I. Fita, M. G. Rossmann, The active center of catalase, J. Mol. Biol. 1985, 185, $21-37$.

[198] T. Wyttenbach, M. Witt, M. T. Bowers, On the stability of amino acid zwitterions in the gas phase: The influence of derivatization, proton affinity, and alkali ion addition, J. Am. Chem. Soc. 2000, 122, 3458-3464.

[199] L. C. Snoek, R. T. Kroemer, J. P. Simons, A spectroscopic and computational exploration of tryptophan-water cluster structures in the gas phase, Phys. Chem. Chem. Phys. 2002, 4, 2130-2139.

[200] F. Schneider, Histidin im aktiven Zentrum von Enzymen, Angew. Chem. 1978, 90, 616-625.

[201] V. Poterya, V. Profant, M. Fárník, L. Šištík, P. Slavíček, U. Buck, Photoinduced processes in hydrogen bonded system: Photodissociation of imidazole clusters, J. Phys. Chem. A 2009, 113, 14583-14590.

[202] B. Narasimhan, D. Sharma, P. Kumar, Biological importance of imidazole nucleus in the new millenium, Med. Chem. Res. 2011, 20, 1119-1140.

[203] J. W. Henderson, P. Haake, Role of water in the imidazole-catalyzed hydrolysis of $p$-nitrotrifluoroacetanilide. A study of solvation in acetonitrile-water mixtures, J. Org. Chem. 1977, 42, 3989-3994. 
[204] M. R. Hockridge, E. G. Robertson, J. P. Simons, Ion-dip spectroscopy and structure of 5-phenyl imidazole, 4-phenyl imidazole and its 1:1 hydrate, Chem. Phys. Lett. 1999, 302, 538-548.

[205] M. R. Hockridge, E. G. Robertson, J. P. Simons, D. R. Borst, T. M. Korter, D. W. Pratt, The $\mathrm{S}_{1} \leftarrow \mathrm{S}_{0}$ electronic transitions of 4- and 5-phenyl imidazole in the gas phase, Chem. Phys. Lett. 2001, 334, 31-38.

[206] F. O. Talbot, J. P. Simons, Infrared ion dip and ultraviolet spectroscopy of 4phenyl imidazole, its tautomer, 5-phenyl imidazole, and its multiply hydrated clusters, Eur. Phys. J. D 2002, 20, 389-398.

[207] C. A. Rice, N. Borho, M. A. Suhm, Dimerization of pyrazole in slit jet expansions, Z. Phys. Chem. 2005, 219, 379-388.

[208] R. L. Ornstein, Y. J. Zheng, Ab initio mechanics analysis of imidazole C-H...O water hydrogen bonding and a molecular mechanics forcefield correction, J. Biomol. Struct. Dyn. 1997, 14, 657-665.

[209] G. Alagona, C. Ghio, Comparative study of imidazole hydration: Ab Initio and electrostatic calculations vs. Cambridge Structural Database analysis, J. Comp. Chem. 1990, 11, 1038-1046.

[210] Y. Matsumoto, K. Honma, Hydrogen-bonded structures of pyrrole-solvent clusters: Infrared cavity ringdown spectroscopy and quantum chemical calculations, J. Chem. Phys. 2009, 130, 054311.

[211] I. Dauster, C. A. Rice, P. Zielke, M. A. Suhm, N-H $\cdots \pi$ interactions in pyrroles: systematic trends from the vibrational spectroscopy of clusters, Phys. Chem. Chem. Phys. 2008, 10, 2827-2835.

[212] H. J. Bakker, J. L. Skinner, Vibrational spectroscopy as a probe of structure and dynamics in liquid water, Chem. Rev. 2010, 110, 1498-1517.

[213] M. K. Van Bael, J. Smets, K. Schoone, L. Houben, W. McCarthy, L. Adamowicz, M. J. Nowak, G. Maes, Matrix-isolation FTIR studies and theoretical calculations of hydrogen-bonded complexes of imidazole. A comparison between experimental results and different calculation methods, J. Phys. Chem. A 1997, 101, 23972413 .

[214] P. I. Nagy, G. J. Durant, D. A. Smith, Theoretical studies on hydration of pyrrole, imidazole, and protonated imidazole in the gas phase and aqueous solution, J. Am. Chem. Soc. 1993, 115, 2912-2922. 
[215] T. H. Dunning Jr., Gaussian basis sets for use in correlated molecular calculations. I. The atoms boron through neon and hydrogen, J. Chem. Phys. 1989, 90, 10071023 .

[216] K. S. Kim, P. Tarakeshwar, J. Y. Lee, Molecular clusters of $\pi$-systems: Theoretical studies of structures, spectra, and origin of interaction energies, Chem. Rev. 2000, 100, 4145-4185.

[217] S. Carles, F. Lecomte, J. P. Schermann, C. Desfrançois, Gas-phase experimental and theoretical studies of adenine, imidazole, pyrrole, and water non-covelent complexes, J. Phys. Chem. A 2000, 104, 10662-10668.

[218] M. Y. Choi, R. E. Miller, Infrared laser spectroscopy of imidazole complexes in helium nanodroplets: Monomer, dimer, and binary water complexes, J. Phys. Chem. A 2006, 110, 9344-9351.

[219] M. Y. Choi, G. E. Douberly, T. M. Falconer, W. K. Lewis, C. M. Lindsay, J. M. Merritt, P. L. Stiles, R. E. Miller, Infrared spectroscopy of helium nanodroplets: novel methods for physics and chemistry, Int. Rev. Phys. Chem. 2006, 25, 15-75.

[220] M. F. H. Schuster, W. H. Meyer, M. Schuster, K. D. Kreuer, Toward a new type of anhydrous organic proton conductor based on immobilized imidazole, Chem. Mater. 2004, 16, 329-337.

[221] B. Bae, H. Y. Ha, D. Kim, Preparation and characterization of Nafion/poly(1vinylimidazole) composite membrane for direct methanol fuel cell application, J. Electrochem. Soc. 2005, 152, A1366-A1372.

[222] W. Y. Sohn, M. Kim, S.-S. Kim, Y. D. Park, H. Kang, Solvent-assisted conformational isomerization and the conformationally-pure REMPI spectrum of 3-aminophenol, Phys. Chem. Chem. Phys 2011, 13, 7037-7042.

[223] U. Erlekam, M. Frankowski, G. von Helden, G. Meijer, Cold collisions catalyse conformational conversion, Phys. Chem. Chem. Phys. 2007, 9, 3786-3789.

[224] I. Ashikawa, K. Itoh, Raman spectra of polypeptides containing L-histidine residues and tautomerism of imidazole side chain, Biopolymers 1979, 18, 1859-1876.

[225] C. Öğretir, S. Yarligan, AM1 and PM3 study of the protonation tautomerization and valence tautomerization of some 4-substituted imidazoles, J. Mol. Struct. (Theochem) 1998, 425, 249-254.

[226] M. T. Güllüoğlu, Y. Erdogdu, J. Karpagam, N. Sundaraganesan, Ş. Yurdakul, DFT, FT-Raman, FT-IR and FT-NMR studies of 4-phenylimidazole, J. Mol. Struct. 2011, 990, 14-20. 
[227] R. M. Claramunt, M. D. Santa María, L. Infantes, F. H. Cano, J. Elguero, The annular tautomerism of 4(5)-phenylimidazole, J. Chem. Soc., Perkin Trans. 2002, 2, 564-568.

[228] M. S. Shaik, S. Y. Liem, Y. Yuan, P. L. A. Popelier, Simulation of liquid imidazole using a high-rank quantum topological electrostatic potential, Phys. Chem. Chem. Phys. 2010, 12, 15040-15055.

[229] A. Zehnacker, M. A. Suhm, Chirality recognition between neutral molecules in the gas phase, Angew. Chem. Int. Ed. 2008, 47, 6970-6992.

[230] M. Mitsuhashi, D. G. Payan, Functional diversity of histamine and histamine receptors, J. Invest. Dermatol. 1992, 98, 8S-11S.

[231] J. Zischang, Konkurrierende Wechselwirkungszentren: Histamin und Imidazol in der Gasphase, Diplomarbeit, Georg-August-Universität Göttingen, 2010.

[232] W. C. Trogler, Physical properties and mechanisms of formation of nitrous oxide, Coord. Chem. Rev. 1999, 187, 303-327.

[233] Z. Cao, X. Gao, L. Deng, W. D. Chen, Y. Yuan, W. Zhang, Z. Gong, A difference frequency generation spectrometer and its detection of atmospheric $\mathrm{N}_{2} \mathrm{O}$, Spectrochim. Acta, Part A 2007, 68, 74-77.

[234] I. Suzuki, General anharmonic force constants of nitrous oxide, J. Mol. Spectrosc. 1969, 32, 54-73.

[235] H. Yamada, W. B. Person, Absolute infrared intensities of some linear triatomic molecules in the gas phase, J. Chem. Phys. 1966, 45, 1861-1865.

[236] M. Bryant, S. W. Reeve, W. A. Burns, Observation and analysis of $\mathrm{N}_{2} \mathrm{O}$ rotationvibration spectra: A physical chemistry laboratory experiment, J. Chem. Educ. 2008, 85, 121-124.

[237] R. H. Kagann, Infrared absorption intensities for $\mathrm{N}_{2} \mathrm{O}$, J. Mol. Spectrosc. 1982 , 95, 297-305.

[238] R. E. Miller, R. O. Watts, A. Ding, Vibrational predissociation spectra of nitrous oxide clusters, Chem. Phys. 1984, 83, 155-169.

[239] J. W. Fleming, Gas phase i.r. absorption spectrometry at extremely long wavelengths $(714 \mu \mathrm{m}-1667 \mu \mathrm{m})$, Spectrochim. Acta 1977, 33A, 787-791.

[240] E. K. Plyler, E. F. Barker, The infrared spectrum and the molecular configuration of $\mathrm{N}_{2} \mathrm{O}$, Phys. Rev. 1931, 38, 1827-1836. 
[241] E. K. Plyler, E. D. Tidwell, H. C. Allen Jr., Near infrared spektrum of nitrous oxide, J. Chem. Phys. 1956, 24, 95-97.

[242] M. K. Kunzmann, R. Signorell, M. Taraschewski, S. Bauerecker, The formation of $\mathrm{N}_{2} \mathrm{O}$ nanoparticles in a collisional cooling cell between 4 and $110 \mathrm{~K}$, Phys. Chem. Chem. Phys. 2001, 3, 3742-3749.

[243] M. K. Kunzmann, S. Bauerecker, M. A. Suhm, R. Signorell, Spectroscopic characterization of $\mathrm{N}_{2} \mathrm{O}$ aggregates: from clusters to the particulate state, Spectrochim. Acta, Part A 2003, 59, 2855-2865.

[244] S. Kudoh, K. Onoda, M. Takayanagi, M. Nakata, $\mathrm{N}_{2} \mathrm{O}$ clusters in supersonic jet studied by matrix-isolation infrared spectroscopy and density functional theory calculation, J. Mol. Struct. 2000, 524, 61-68.

[245] A. W. Liu, S. Kassi, P. Malara, D. Romanini, V. I. Perevalov, S. A. Tashkun, S. M. Hu, A. Campargue, High sensitivity $C W$-cavity ring down spectroscopy of $\mathrm{N}_{2} \mathrm{O}$ near $1.5 \mu \mathrm{m}$ (I), J. Mol. Spectrosc. 2007, 244, 33-47.

[246] M. Gauthier, Spectra and structure of nitrous oxide dimers and clusters, J. Chem. Phys. 1988, 88, 5439-5449.

[247] T. E. Gough, R. E. Miller, G. Scoles, Photo-induced vibrational predissociation of the van der Waals molecule $\left(\mathrm{N}_{2} \mathrm{O}\right)_{2}$, J. Chem. Phys. 1978, 69, 1588-1590.

[248] M. Dehghany, M. Afshari, N. Moazzen-Ahmadi, A. R. W. McKellar, Combination bands of the $\mathrm{N}_{2} \mathrm{O}$ trimer involving the intermolecular modes in the $2260 \mathrm{~cm}^{-1}$ region, Chem. Phys. Lett. 2009, 476, 143-146.

[249] R. E. Miller, L. Pedersen, The infrared spectrum and structure of the nitrous oxide trimer, J. Chem. Phys. 1998, 108, 436-443.

[250] A. D. Buckingham, C. Graham, J. H. Williams, Electric field-gradient-induced birefringence in $\mathrm{N}_{2}, \mathrm{C}_{2} \mathrm{H}_{6}, \mathrm{C}_{3} \mathrm{H}_{6}, \mathrm{Cl}_{2}, \mathrm{~N}_{2} \mathrm{O}$ and $\mathrm{CH}_{3} \mathrm{~F}$, Mol. Phys. 1983, 49, 703-710.

[251] T. Häber, R. Kevorkiants, W. Thiel, M. A. Suhm, The performance of the semiempirical AM1 method on small and nanometre-sized $\mathrm{N}_{2} \mathrm{O}$ clusters, Phys. Chem. Chem. Phys. 2004, 6, 4939-4949.

[252] R. Matsushima, T. Ebata, Y. Inokuchi, Formation of semi-covalent bond in $\left[\left(\mathrm{N}_{2} \mathrm{O}\right)_{n} \mathrm{H}_{2} \mathrm{O}\right]^{+}(n=2-7)$ cluster ions studied by IR spectroscopy, J. Phys. Chem. A 2010, 114, 11037-11042.

[253] A. R. W. McKellar, Infrared spectra of helium clusters seeded with nitrous oxide, ${ }^{4} \mathrm{He}_{N^{-}}-\mathrm{N}_{2} \mathrm{O}$, with $N=1-80, J$. Chem. Phys. 2007, $127,044315$. 
[254] H. Sato, S. Sakaki, Y. Sakamoto, M. Kawasaki, Effective interaction energies for weakly bound dimers at room temperature: $\left(\mathrm{H}_{2} \mathrm{O}\right)_{2},\left(\mathrm{~N}_{2} \mathrm{O}\right)_{2},\left(\mathrm{CO}_{2}\right)_{2}$, and $(\mathrm{HCHO})_{2}$, Chem. Lett. 2010, 39, 296-297.

[255] J. Plíva, Infrared spectra of isotopic nitrous oxide, J. Mol. Spectrosc. 1961, 12, 360-386.

[256] D. E. Burch, E. B. Singleton, D. Williams, Absorption line broadening in the infrared, Appl. Opt. 1962, 1, 359-363.

[257] P. C. Cross, F. Daniels, The influence of foreign gases of the intensity of infrared absorption, J. Chem. Phys. 1934, 2, 6-10.

[258] J. Zischang, M. A. Suhm, The OH stretching spectrum of warm water clusters, J. Chem. Phys. 2014, 140, 064312. 


\section{Lebenslauf}

\section{Persönliche Daten}

Vor- und Nachname Julia Zischang

Geburtstag und -ort 24.05.1985, Celle

Staatsangehörigkeit deutsch

\section{Ausbildung}

\begin{tabular}{|c|c|}
\hline 08/1992-07/1996 & Grundschule Wrestedt \\
\hline 08/1996-08/1998 & Orientierungsstufe Bad Bodenteich \\
\hline 09/1998-06/2005 & Herzog-Ernst-Gymnasium Uelzen \\
\hline $06 / 2005$ & $\begin{array}{l}\text { Allgemeine Hochschulreife (Abitur) } \\
\text { Herzog-Ernst-Gymnasium Uelzen }\end{array}$ \\
\hline $10 / 2005-03 / 2010$ & $\begin{array}{l}\text { Diplomstudiengang Chemie } \\
\text { Georg-August-Universität Göttingen }\end{array}$ \\
\hline $10 / 2007$ & $\begin{array}{l}\text { Vordiplom Chemie } \\
\text { Georg-August-Universität Göttingen }\end{array}$ \\
\hline $09 / 2009-01 / 2010$ & $\begin{array}{l}\text { Diplomarbeit, Konkurrierende Wechselwirkungszentren: } \\
\text { Histamin und Imidazol in der Gasphase } \\
\text { Institut für Physikalische Chemie (Prof. Dr. M. A. Suhm) } \\
\text { Georg-August-Universität Göttingen }\end{array}$ \\
\hline $03 / 2010$ & $\begin{array}{l}\text { Diplom Chemie } \\
\text { Georg-August-Universität Göttingen }\end{array}$ \\
\hline seit $05 / 2010$ & $\begin{array}{l}\text { Promotion, Strukturdynamik wasserhaltiger Aggregate } \\
\text { in Überschallexpansionen } \\
\text { Institut für Physikalische Chemie (Prof. Dr. M. A. Suhm) } \\
\text { Georg-August-Universität Göttingen }\end{array}$ \\
\hline
\end{tabular}

